\title{
Access to Z-Selective 1,3-Enynes via Ni-Catalyzed Intermolecular Cross-Alkylalkynylation of Terminal Alkynes
}

Nan Xiao, ${ }^{\dagger, \|}$ Yi-Zhou Zhan, ${ }^{\dagger, \|}$ Huan Meng, ${ }^{\dagger, l}$ and Wei Shu ${ }^{*}, \dagger$

${ }^{\dagger}$ Shenzhen Grubbs Institute, Department of Chemistry, and Guangdong Provincial Key Laboratory of Catalysis, Southern University of Science and Technology, 518055 Shenzhen, Guangdong, China

*E-mail: shuw@sustech.edu.cn

\section{Supporting Information}

\section{Table of Contents}

1. General Information $\quad$ S2

2. Tables of the Optimization of Reaction Conditions S3

3. General Procedures $\quad$ S9

4. Characterization of New Compounds S11

$\begin{array}{ll}\text { 5. Mechanistic Experiments } & \text { S27 }\end{array}$

$\begin{array}{ll}\text { 6. Reference } & \text { S30 }\end{array}$

7. ${ }^{1} \mathrm{H},{ }^{13} \mathrm{C}$, and ${ }^{19} \mathrm{~F}$ Spectra of New Compounds S31

8. X-Ray Diffraction Data of 7l S86 


\section{General Information}

NMR spectra were recorded on $400 \mathrm{MHz}$ or $600 \mathrm{MHz}$ Bruker spectrometers. Chemical shifts are given in ppm. The spectra are calibrated to the residual ${ }^{1} \mathrm{H}$ and ${ }^{13} \mathrm{C}$ signals of the solvents. Multiplicities are abbreviated as follows: singlet (s), doublet (d), triplet (t), quartet (q), doublet-doublet (dd), quintet (quint), septet (sept), multiplet (m), and broad (br). High-resolution electrospray ionization and electronic impact mass spectrometry was performed on a Finnigan MAT 900 (Thermo Finnigan, San Jose, CA; USA) double focusing magnetic sector mass spectrometer (mass analyzer type: Orbitrap). A mass accuracy $\leq 2 \mathrm{ppm}$ was obtained in the peak matching acquisition mode by using a solution containing $2<1$ PEG200, $2<1$ PPG450, and $1.5 \mathrm{mg} \mathrm{NaOAc}$ (all obtained from Sigma-Aldrich, CH-Buchs) dissolved in 100 mL MeOH (HPLC Supra grade, Scharlau, E-Barcelona) as internal standard.

Materials and methods: Unless otherwise stated, starting materials were used upon purchased. Solvents were purchased in HPLC quality, degassed by purging with nitrogen and dried over activated molecular sieves of appropriate size. Alternatively, they were purged with argon and passed through alumina columns in a solvent purification system (Innovative Technology). Conversion was monitored by thin layer chromatography (TLC) using Merck TLC silica gel 60 F254. Compounds were visualized by UV light at $254 \mathrm{~nm}$ and by dipping the plates in an ethanolic vanillin/sulfuric acid solution or an aqueous potassium permanganate solution followed by heating. Flash column chromatography was performed over silica gel (300-400 mesh). 


\section{Tables of the Optimization of Reaction Conditions}

Table S1. Screening of Loading Amount of 1-3

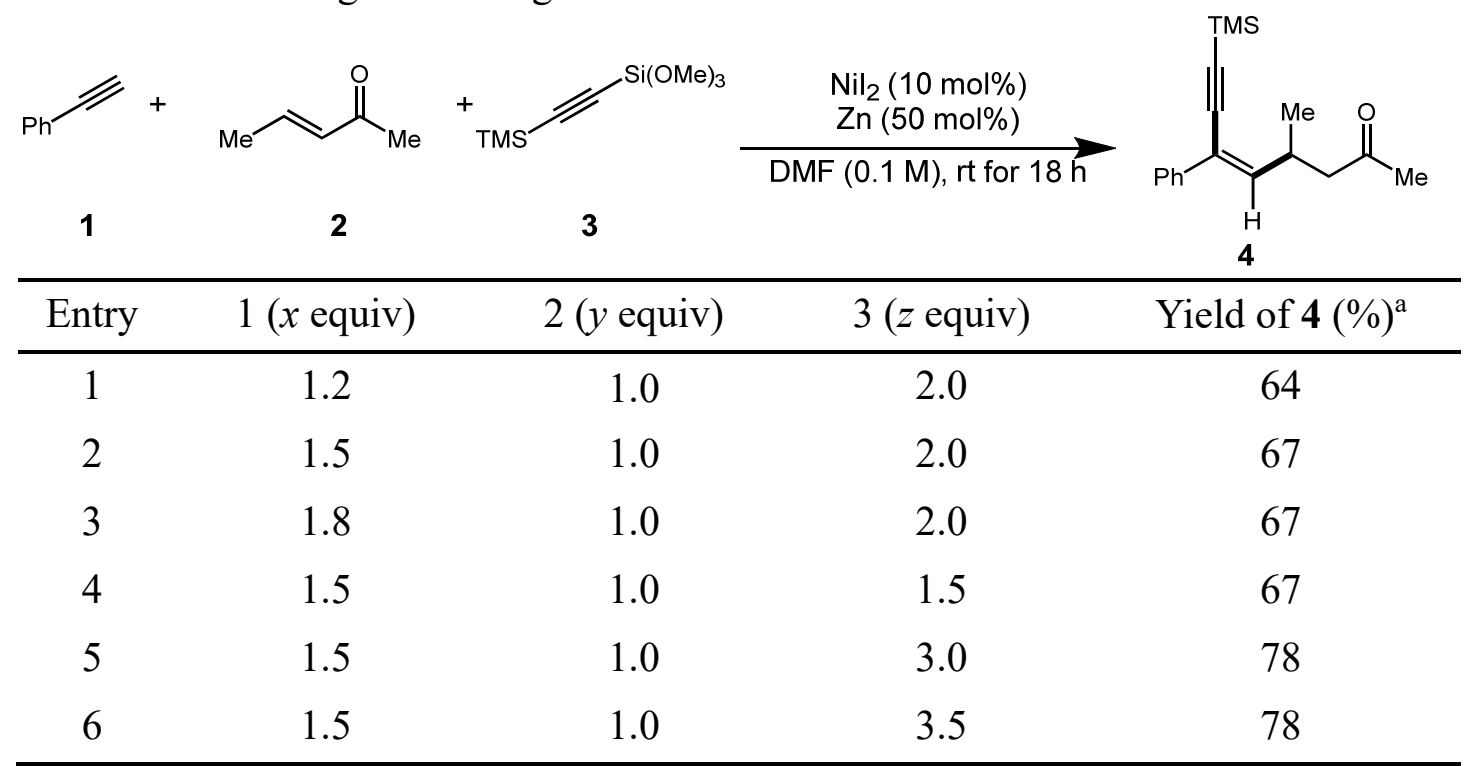

${ }^{\mathrm{a}}$ Reaction was run using 2 (0.20 mmol, 1.0 equiv), $\mathbf{1}$ (1.2-1.8 equiv) and $\mathbf{3}$ (1.5-3.5 equiv) under indicated conditions for $18 \mathrm{~h}$. Yield was determined by $\mathrm{GC}$ analysis using $n$-dodecane as internal standard.

Table S2. Screening of Reductant

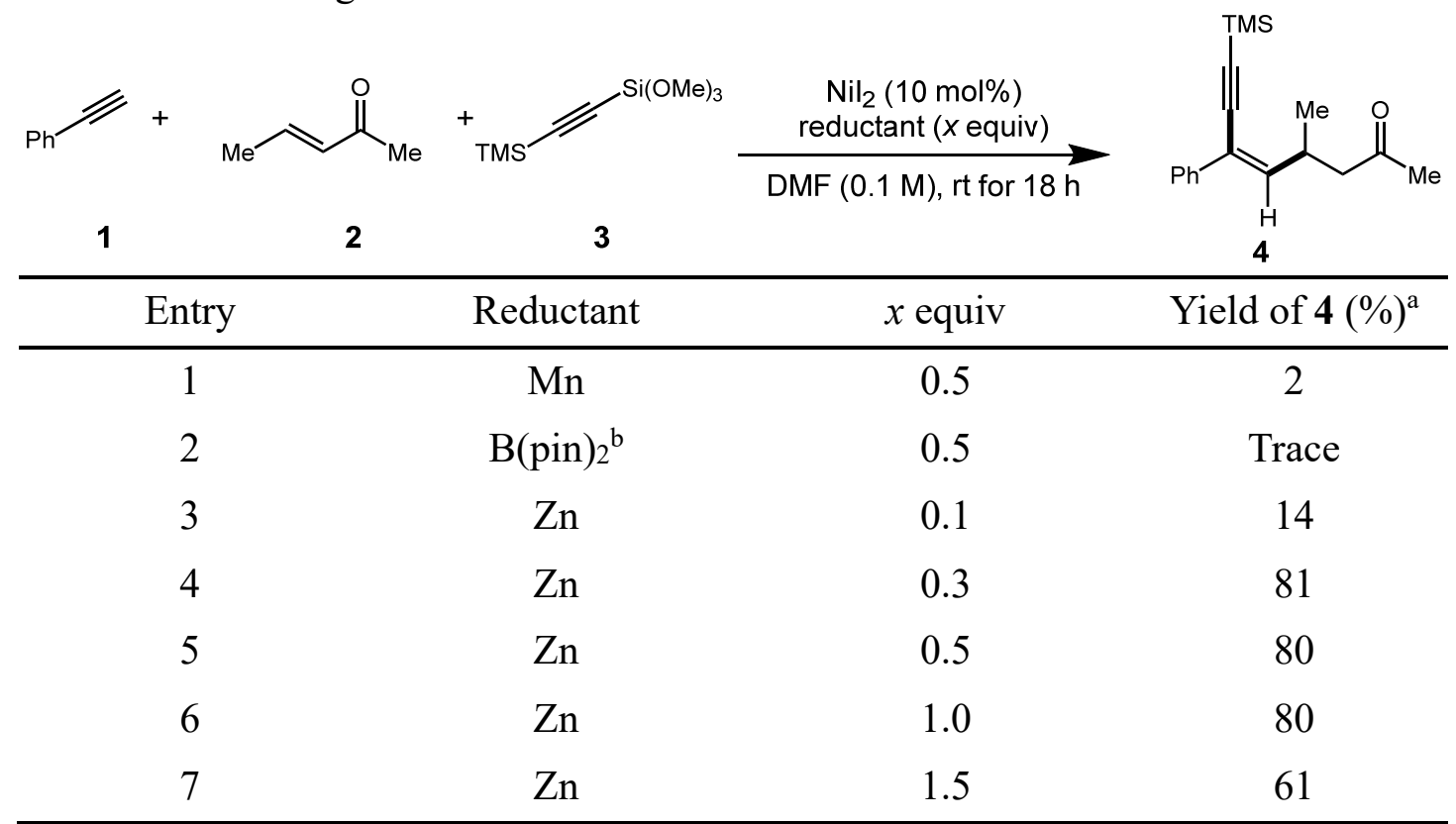

${ }^{\text {a Reaction was run using } 0.30 \mathrm{mmol} \text { of } \mathbf{1}, 0.20 \mathrm{mmol} \text { of } 2 \text { and } 0.60 \mathrm{mmol} \text { of } \mathbf{3} \text { under }}$ indicated conditions for $18 \mathrm{~h}$. Yield was determined by GC analysis using $n$-dodecane as internal standard. ${ }^{\mathrm{b}} \mathrm{NaO} \mathrm{Bu}^{t}(0.05 \mathrm{mmol})$ was added.

Table S3. Screening of Proton Source 


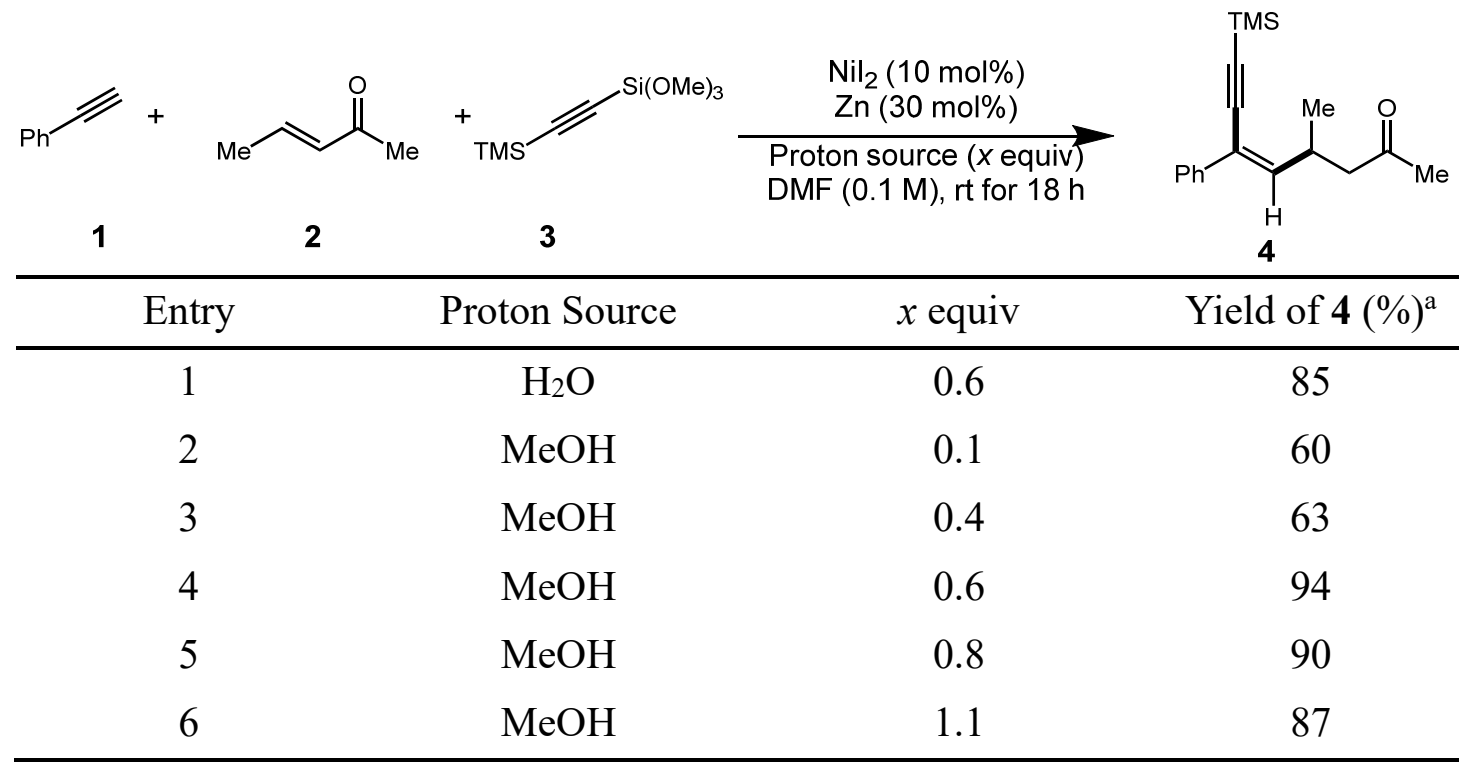

a Reaction was run using $0.30 \mathrm{mmol}$ of $1,0.20 \mathrm{mmol}$ of 2 and $0.60 \mathrm{mmol}$ of 3 under indicated conditions for $18 \mathrm{~h}$. Yield was determined by GC analysis using $n$-dodecane as internal standard.

Table S4. Screening of Solvent

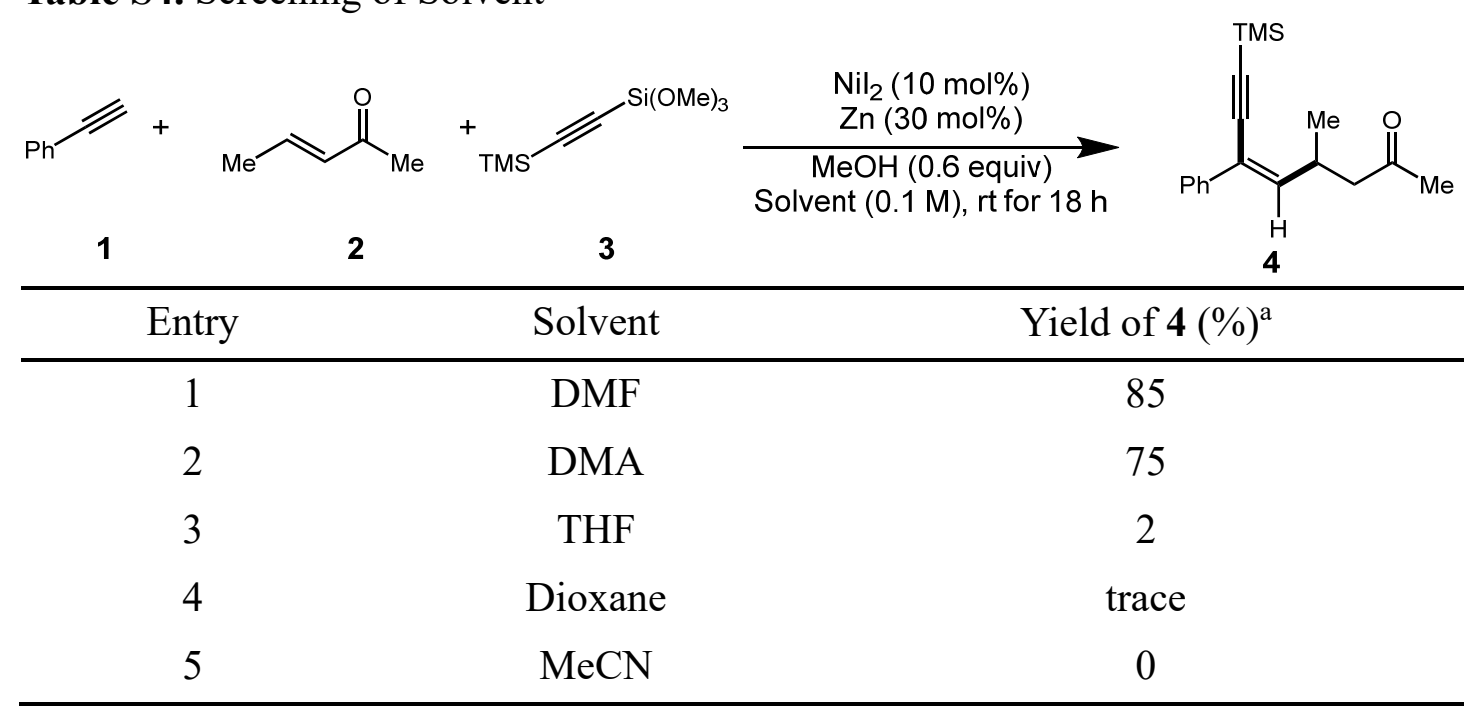

${ }^{a}$ Reaction was run using $0.30 \mathrm{mmol}$ of $\mathbf{1}, 0.20 \mathrm{mmol}$ of 2 and $0.60 \mathrm{mmol}$ of $\mathbf{3}$ under indicated conditions for $18 \mathrm{~h}$. Yield was determined by GC analysis using $n$-dodecane as internal standard.

Table S5. Ni-Source Screening

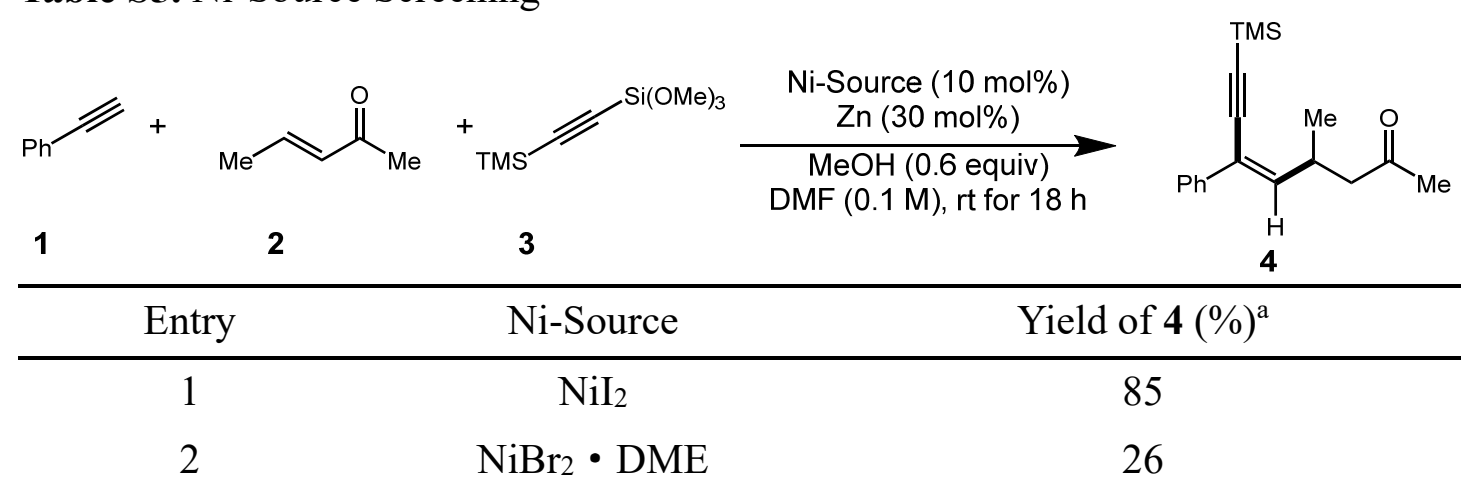




$\begin{array}{ccc}3 & \mathrm{NiCl}_{2} & 20 \\ 4 & \mathrm{NiBr}_{2} & 23 \\ 5 & \mathrm{Ni}(\mathrm{OAc})_{2} & 0 \\ 6 & \mathrm{Ni}(\mathrm{acac})_{2} & \text { trace } \\ 7 & \mathrm{Ni}\left(\mathrm{ClO}_{4}\right)_{2} \cdot 6 \mathrm{H}_{2} \mathrm{O} & \text { trace }\end{array}$

$\overline{{ }^{a}}$ Reaction was run using $0.30 \mathrm{mmol}$ of $\mathbf{1}, 0.20 \mathrm{mmol}$ of 2 and $0.60 \mathrm{mmol}$ of $\mathbf{3}$ under indicated conditions for $18 \mathrm{~h}$. Yield was determined by GC analysis using $n$-dodecane as internal standard.

Table S6. Ligands Screening

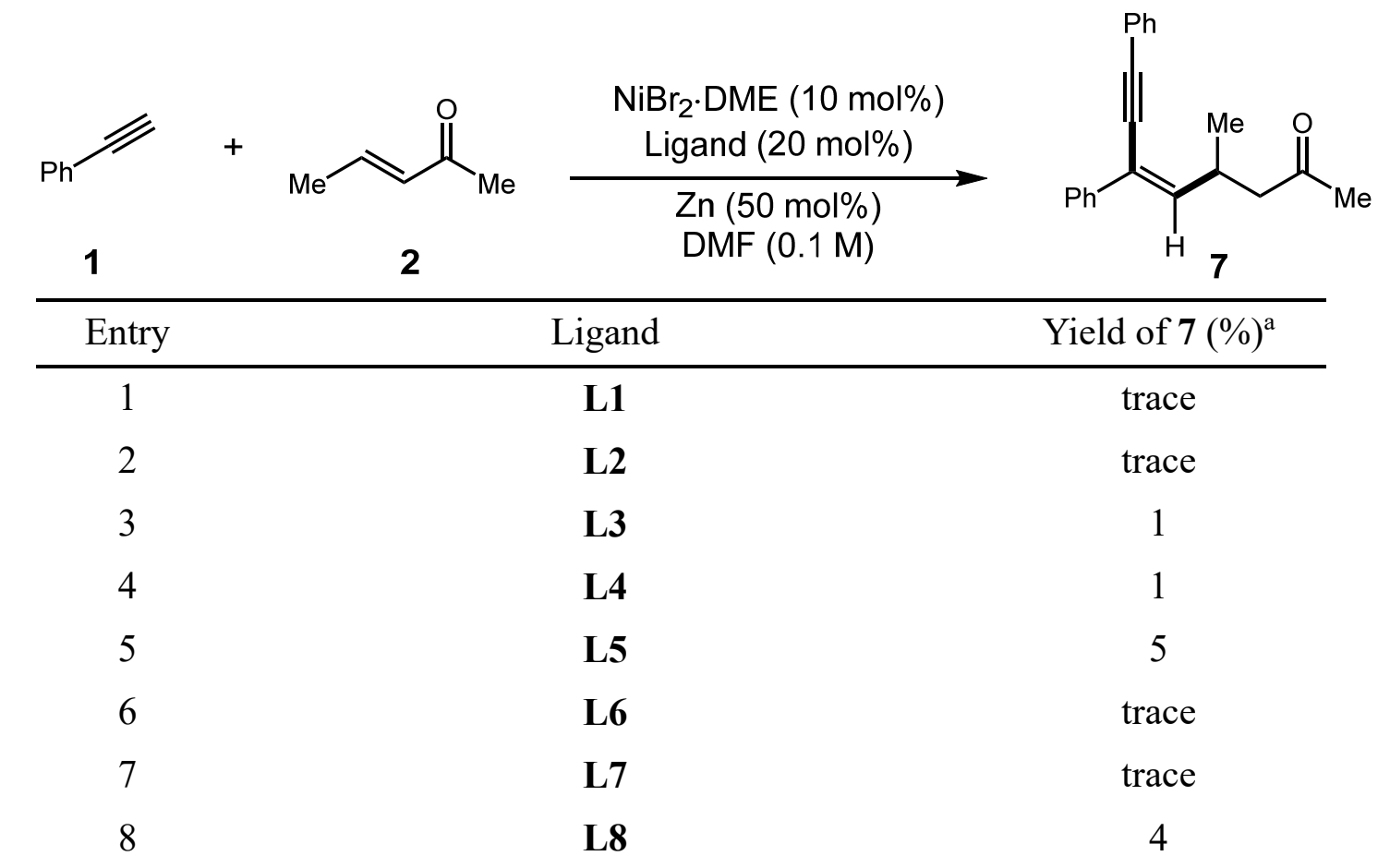

${ }^{\mathrm{a}}$ Reaction was run using $0.80 \mathrm{mmol}$ of 1 and $0.20 \mathrm{mmol}$ of 2 under indicated conditions for $18 \mathrm{~h}$. Yield was determined by GC analysis using $n$-dodecane as internal standard.<smiles>c1cnc2c(c1)ccc1cccnc12</smiles>

L1<smiles>N#Cc1ccncc1</smiles>

L5<smiles>c1ccc(-c2ccccn2)nc1</smiles>

L2<smiles>N#Cc1ccccn1</smiles>

L6<smiles>Br[C@H]1COC(C2=N[17CH]CO2)=N1</smiles>

L3<smiles>N#Cc1cc([N+](=O)[O-])ccn1</smiles>

L7<smiles>Fc1cc(F)cc(-c2cccc([As])n2)c1</smiles>

L4<smiles>Fc1cccnc1</smiles>

L8 
Table S7. Base Screening

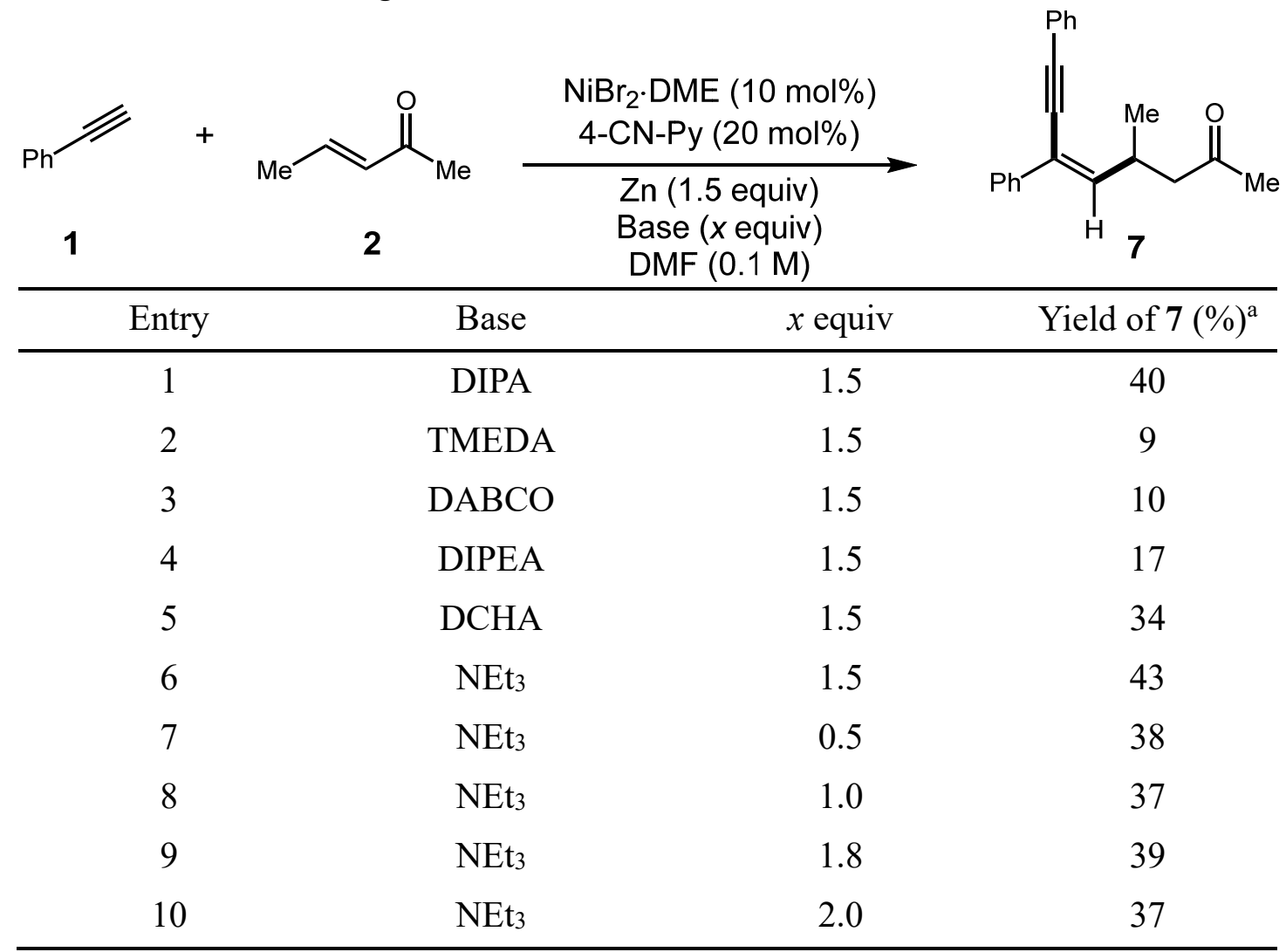

${ }^{a}$ Reaction was run using $0.80 \mathrm{mmol}$ of 1 and $0.20 \mathrm{mmol}$ of 2 under indicated conditions for $18 \mathrm{~h}$. Yield was determined by GC analysis using $n$-dodecane as internal standard.

Table S8. Screening of Loading Amount of Zn

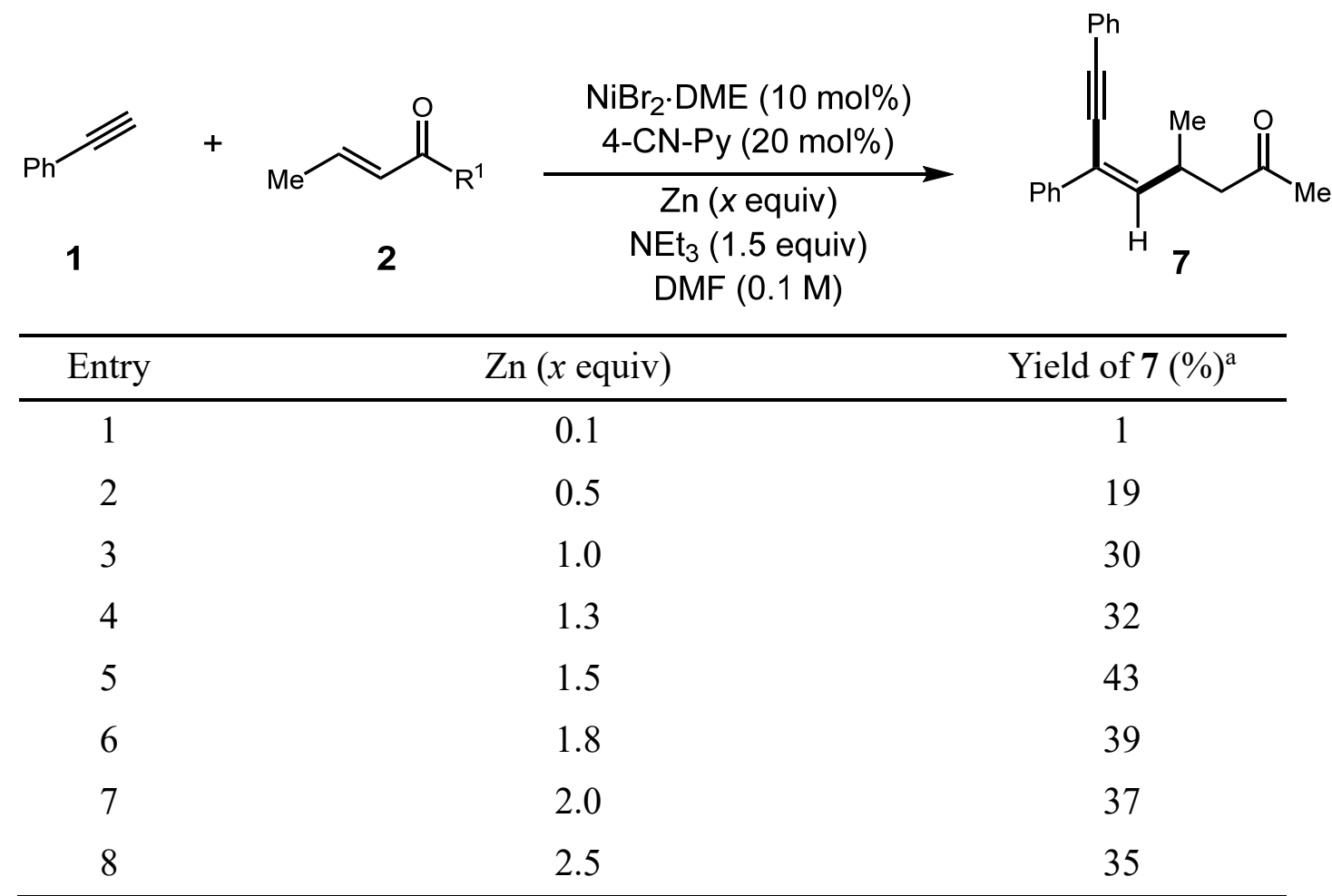

${ }^{\mathrm{a}}$ Reaction was run using $0.80 \mathrm{mmol}$ of 1 and $0.20 \mathrm{mmol}$ of 2 under indicated conditions for $18 \mathrm{~h}$. Yield was determined by GC analysis using $n$-dodecane as internal standard. 
Table S9. Screening of Reaction Concentration
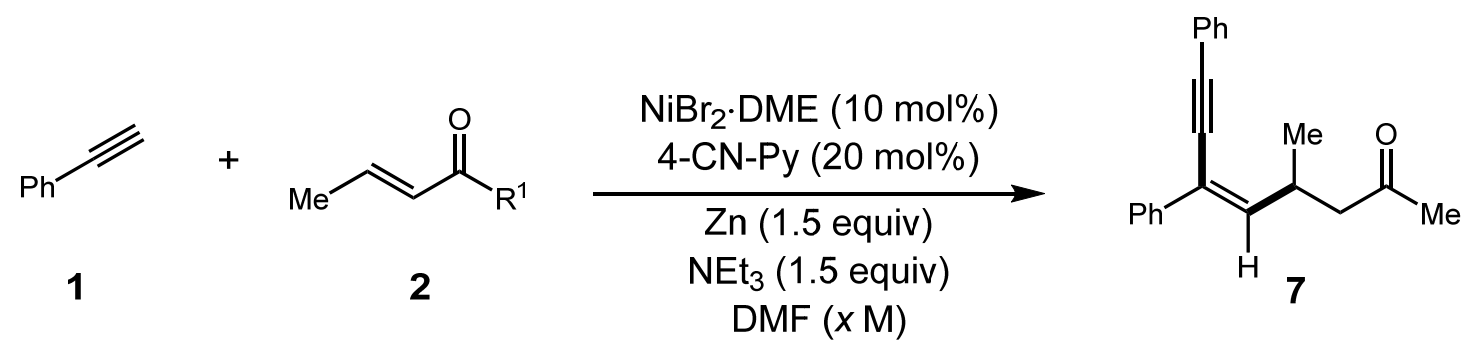

\begin{tabular}{ccc}
\hline Entry & DMF $(x \mathrm{M})$ & ${\text { Yield of } 7(\%)^{\mathrm{a}}}^{\mathrm{a}}$ \\
\hline 1 & 0.05 & 1 \\
2 & 0.10 & 43 \\
3 & 0.15 & 49 \\
4 & 0.20 & 54 \\
5 & 0.23 & 61 \\
6 & 0.25 & $67(59)^{\mathrm{b}}$ \\
7 & 0.28 & 65 \\
8 & 0.30 & 65 \\
\hline
\end{tabular}

${ }^{\mathrm{a}}$ Reaction was run using $0.80 \mathrm{mmol}$ of $\mathbf{1}$ and $0.20 \mathrm{mmol}$ of 2 under indicated conditions for $18 \mathrm{~h}$. Yield was determined by GC analysis using $n$-dodecane as internal standard. ${ }^{\mathrm{b}}$ Isolated yield.

Table S10. Screening of Solvents

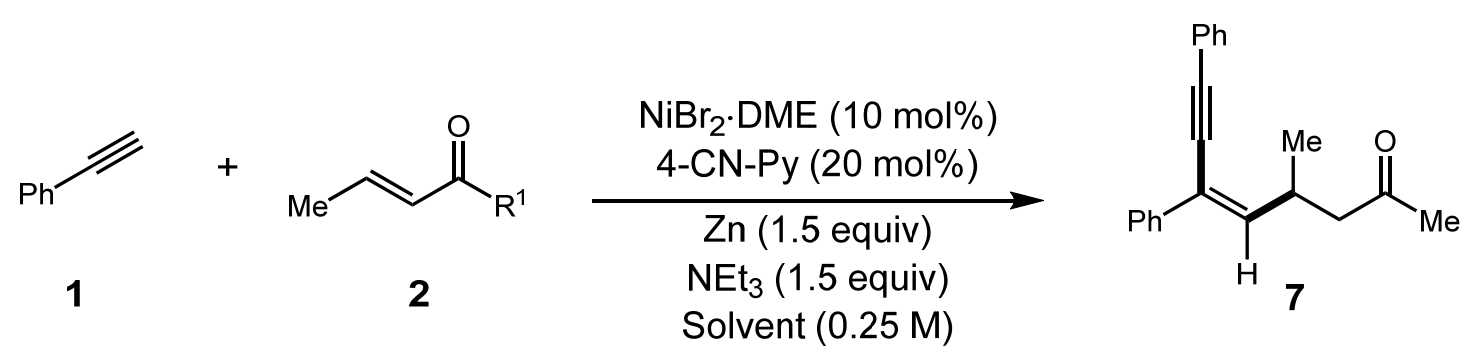

\begin{tabular}{ccc}
\hline Entry & Solvent & ${\text { Yield of } 7(\%)^{\mathrm{a}}}^{\mathrm{a}}$ \\
\hline 1 & DCM & 0 \\
2 & ACN & 0 \\
3 & THF & 0 \\
4 & Dioxane & 0 \\
5 & Toluene & 0 \\
6 & NMP & 47 \\
7 & DMAC & 36 \\
8 & DMF & 67 \\
\hline
\end{tabular}

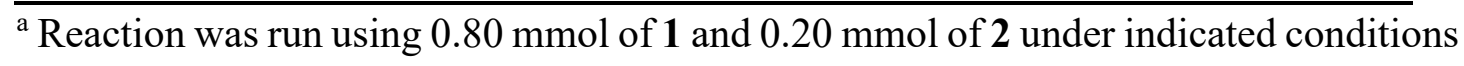
for $18 \mathrm{~h}$. Yield was determined by GC analysis using $n$-dodecane as internal standard. 
Table S11. Ni-Source Screening

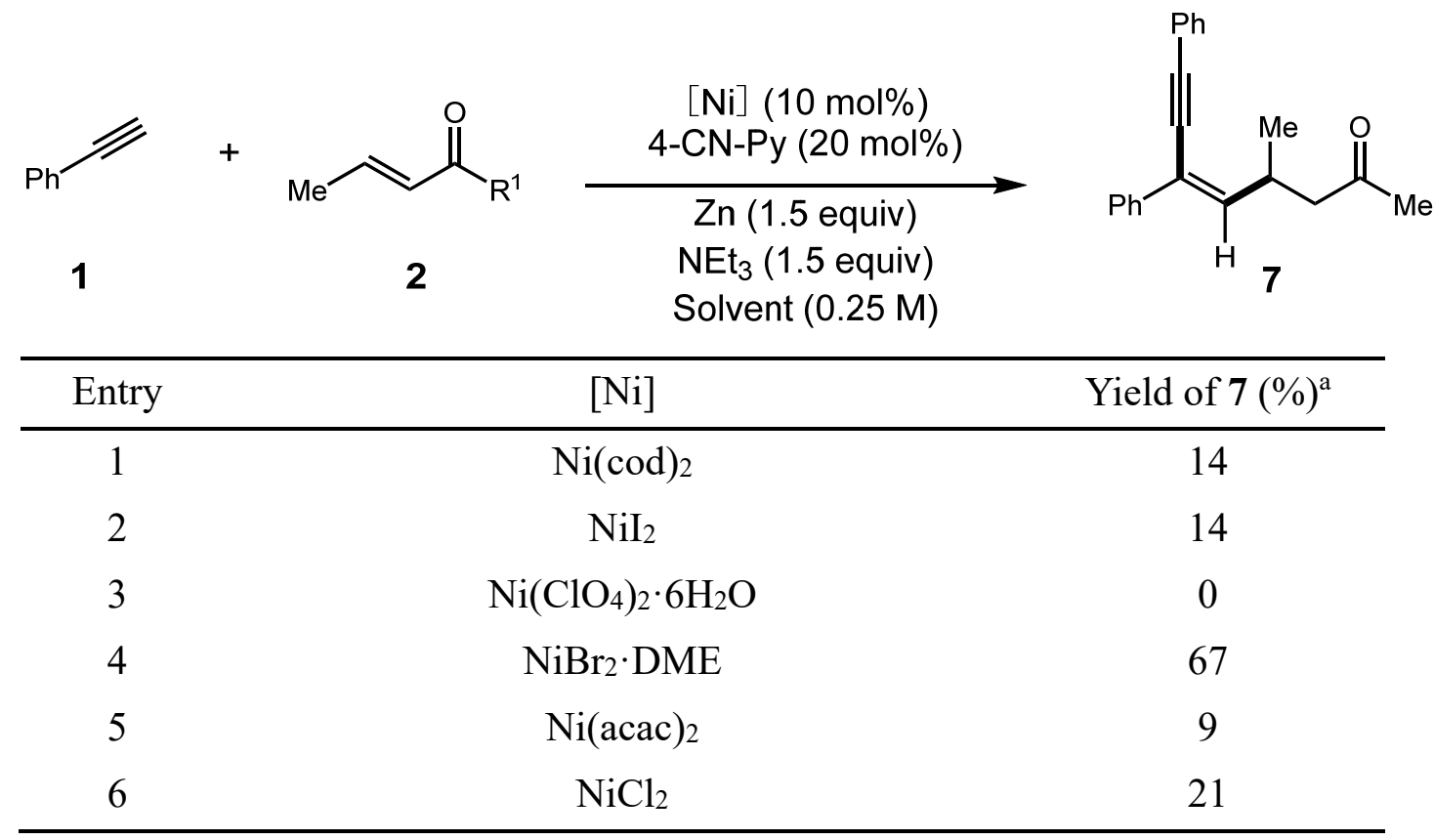

${ }^{\mathrm{a}}$ Reaction was run using $0.80 \mathrm{mmol}$ of 1 and $0.20 \mathrm{mmol}$ of 2 under indicated conditions for $18 \mathrm{~h}$. Yield was determined by GC analysis using $n$-dodecane as internal standard.

Table S12. Screening of Additives

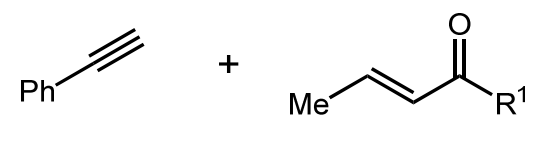

1

2

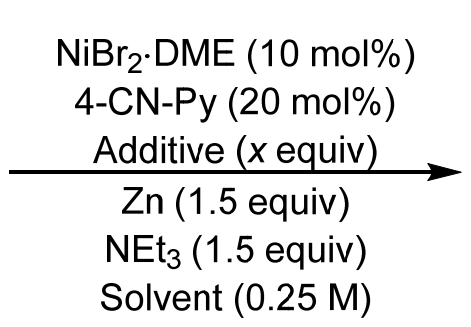

Solvent $(0.25 \mathrm{M})$

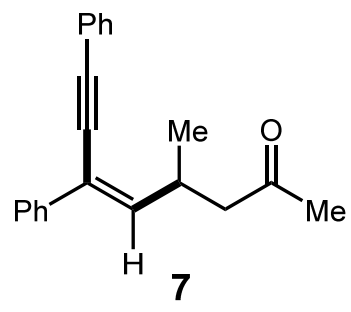

\begin{tabular}{cccc}
\hline Entry & Additive & $x$ equiv & ${\text { Yield of } 7(\%)^{\mathrm{a}}}^{\circ}$ \\
\hline 1 & $\mathrm{MgCl}_{2}$ & 0.2 & 40 \\
2 & $\mathrm{LiBr}$ & 0.3 & 66 \\
3 & $\mathrm{TBAI}$ & 0.3 & 37 \\
4 & $\mathrm{NaI}$ & 0.3 & 67 \\
5 & $\mathrm{KI}$ & 0.3 & 63 \\
6 & $\mathrm{LiI}$ & 0.3 & 80 \\
7 & $\mathrm{LiI}$ & 0.1 & 83 \\
8 & $\mathrm{LiI}$ & 0.5 & 49 \\
9 & LiI & 1.0 & 50 \\
10 & LiI & 2.0 & 30 \\
\hline
\end{tabular}

$\overline{{ }^{a}}$ Reaction was run using $0.80 \mathrm{mmol}$ of 1 and $0.20 \mathrm{mmol}$ of 2 under indicated conditions for $18 \mathrm{~h}$. Yield was determined by GC analysis using $n$-dodecane as internal standard. 
Scheme S1. Unsuccessful substrates

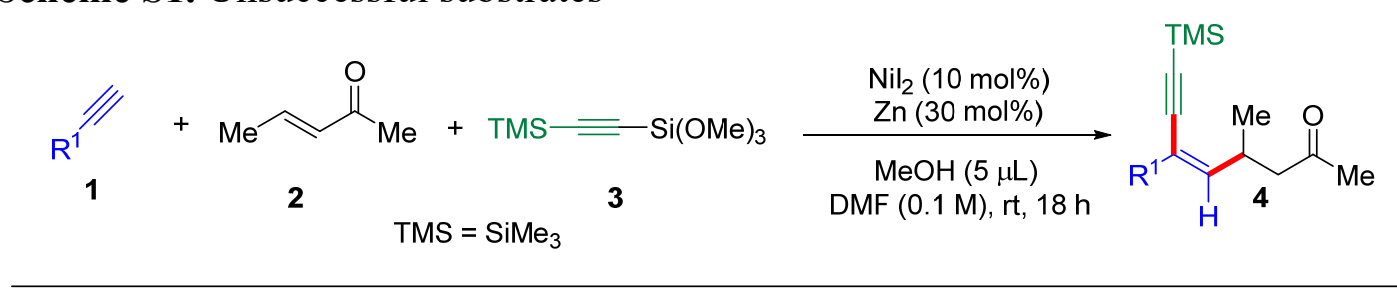

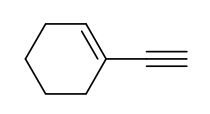

No Reaction

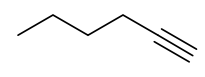

No Reaction

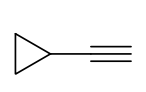

No Reaction

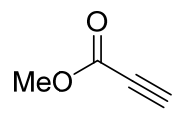

No Reaction

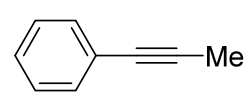

No Reaction

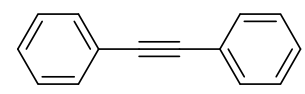

No Reaction
TMS $=$

yield $<30 \%$

${ }^{a}$ Reaction was run using $0.30 \mathrm{mmol}$ of $\mathbf{1}, 0.20 \mathrm{mmol}$ of 2 and $0.60 \mathrm{mmol}$ of $\mathbf{3}$ under indicated conditions for $18 \mathrm{~h}$. Yield was determined by GC analysis using $n$-dodecane as internal standard.

\section{General Procedures}

\subsection{Procedure for the synthesis of alkynyl silicates}

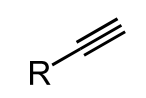

$(26.4 \mathrm{mmol})$
1) $\mathrm{EtMgBr}\left(0.91\right.$ equiv), $0^{\circ} \mathrm{C}, 40^{\circ} \mathrm{C}$ for $2 \mathrm{~h}$

2) $\mathrm{Si}(\mathrm{OMe})_{4}\left(1.36\right.$ equiv), $0^{\circ} \mathrm{C}, 70^{\circ} \mathrm{C}$, overnight

$\operatorname{THF}(0.6 \mathrm{M})$<smiles>[R]C#C[SiH2]C</smiles>

Slicates were synthesized according to literature procedure. ${ }^{1}$ To a solution of alkynes (26.4 mmol, 1.0 equiv) in THF (20 mL) was slowly added ethylmagnesium bromide (1.0 equiv, $1.0 \mathrm{M}$ solution in THF) at $0{ }^{\circ} \mathrm{C}$ under $\mathrm{N}_{2}$, then the mixture was heated up to $40{ }^{\circ} \mathrm{C}$ in an oil bath and stirred for $2 \mathrm{~h}$. After cooling to room temperature, tetramethoxysilane $(5.36 \mathrm{~mL}, 36 \mathrm{mmol}, 1.36$ equiv) was added into the reaction mixture at $0{ }^{\circ} \mathrm{C}$ and the reaction was heated up to $70^{\circ} \mathrm{C}$ in an oil bath and stirred for overnight. Cooling to room temperature, THF was removed and added dry $\mathrm{Et}_{2} \mathrm{O}(30 \mathrm{~mL})$ to the residue, the mixture was then stirred at room temperature for $1 \mathrm{~h}$. Solid was filtered out and $\mathrm{Et}_{2} \mathrm{O}$ was removed. The crude mixture was purified from the residue by distillation under high vacuum to give the corresponding product in pure form. (Colorless oil).

\subsection{Synthesis and Characterization of Alkynyl Silanes}

Typical procedure: Method A: 


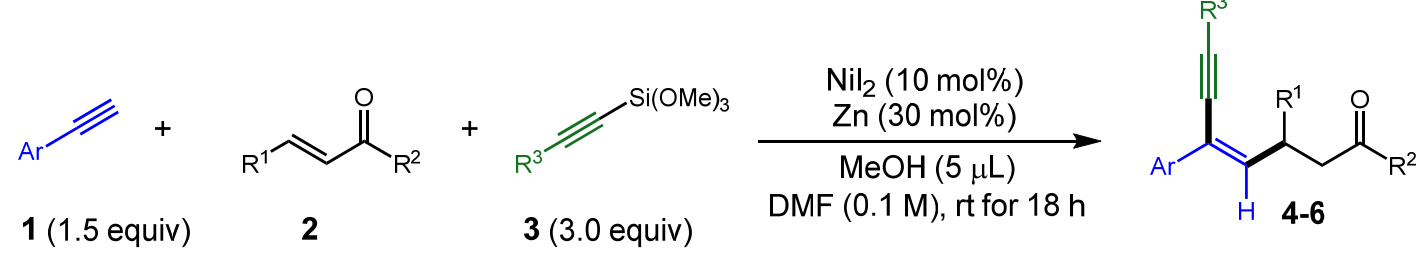

To a Schlenk tube with screw-cap was added NiI2 (6.3 mg, $0.02 \mathrm{mmol}), \mathrm{Zn}(3.9 \mathrm{mg}$, $0.06 \mathrm{mmol})$ and 1 (0.30 mmol, if solid). The tube was evacuated and back-filled with nitrogen (this process was repeated three times). Then DMF $(2.0 \mathrm{~mL}), 3(0.60 \mathrm{mmol})$, $2(0.20 \mathrm{mmol}), \mathbf{1}$ (if liquid), and $\mathrm{MeOH}(5.0 \mu \mathrm{L})$ were added consecutively via syringe. The resulting mixture was stirred at room temperature for $18 \mathrm{~h}$. The reaction mixture was quenched with $\mathrm{H}_{2} \mathrm{O}$, extracted with ethyl acetate $(60 \mathrm{~mL})$. The combined organic phase was washed with saturated brine $(3 \times 30 \mathrm{~mL})$, dried over anhydrous sodium sulfate, filtered and concentrated under reduced pressure. The crude mixture was purified by column chromatography on silica gel with hexane/ethyl acetate mixtures as eluent to give the corresponding product 4-6 in pure form.

Typical procedure: Method B:

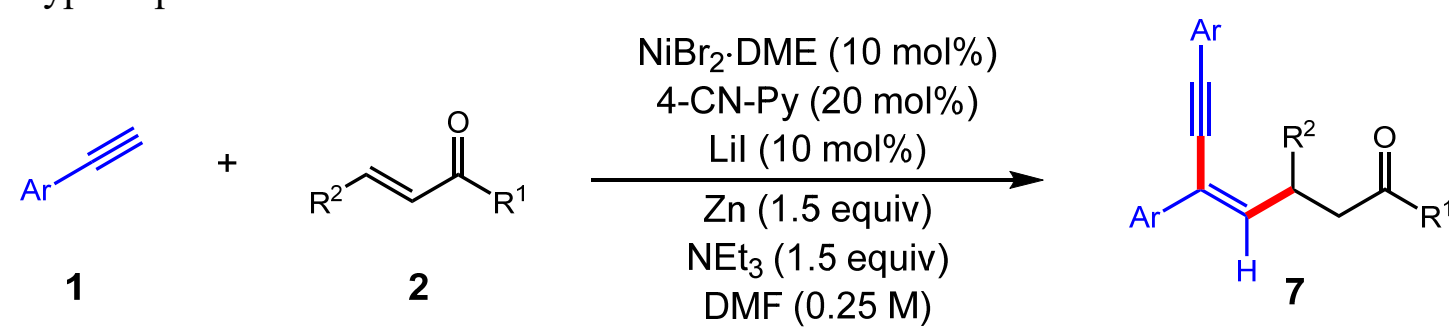

To a Schlenk tube with screw-cap was added $\mathrm{NiBr}_{2}$.DME (6.2 mg, $\left.0.02 \mathrm{mmol}\right), \mathrm{Zn}$ (19.5 mg, $0.30 \mathrm{mmol}$ ), 4-cyanopyridine (4.2 mg, $0.04 \mathrm{mmol})$, LiI (2.7 mg, $0.02 \mathrm{mmol}$ ) and $1(0.80 \mathrm{mmol}$, if solid). The tube was evacuated and back-filled with nitrogen (this process was repeated three times). Then DMF $(0.8 \mathrm{~mL}), \mathbf{2}(0.20 \mathrm{mmol}), \mathbf{1}$ (if liquid) and triethylamine $(42.2 \mu \mathrm{L}, 0.30 \mathrm{mmol})$ were added consecutively via syringe. The resulting mixture was stirred at room temperature for $18 \mathrm{~h}$. The reaction mixture was quenched with $\mathrm{H}_{2} \mathrm{O}$, extracted with ethyl acetate $(60 \mathrm{~mL})$. The combined organic phase was washed with saturated brine $(3 \times 30 \mathrm{~mL})$, dried over anhydrous sodium sulfate, filtered and concentrated under reduced pressure. The crude mixture was purified by column chromatography on silica gel with hexane/ethyl acetate mixtures as eluent to give the corresponding product 7 in pure form. 


\subsection{Procedure for the synthesis of 1,3-enyne $7 \mathrm{~d}$ on $1.0 \mathrm{mmol}$ scale}

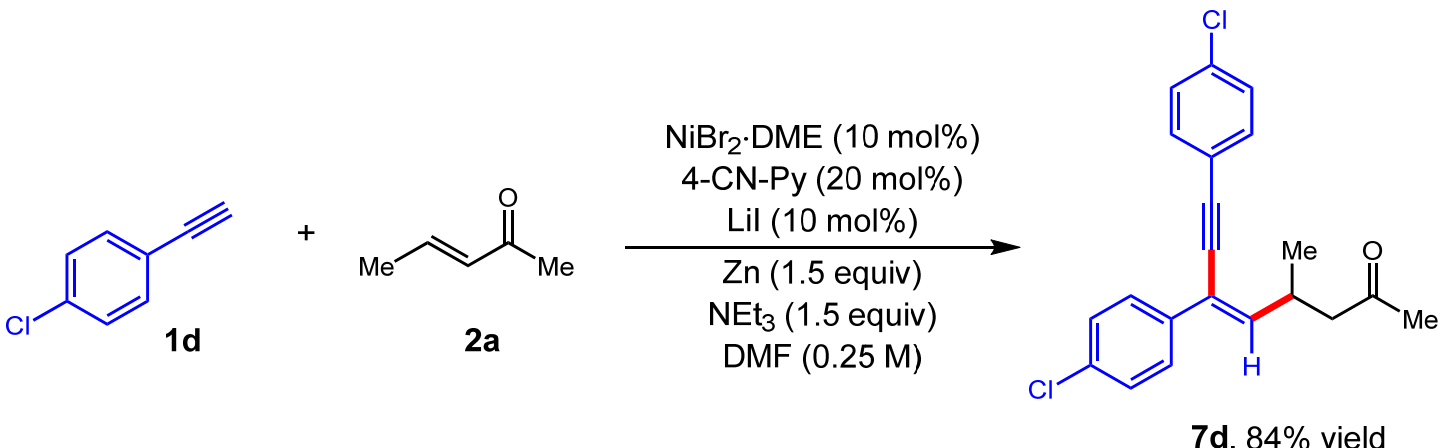

To a Schlenk tube with screw-cap was added NiBr2.DME (30.8 mg, $0.1 \mathrm{mmol}), \mathrm{Zn}$ (97.5 mg, $1.5 \mathrm{mmol})$, 4-cyanopyridine (21.0 mg, $0.2 \mathrm{mmol})$, LiI (13.5 mg, $0.1 \mathrm{mmol})$ and $1 \mathrm{~d}(546.3 \mathrm{mg}, 4.0 \mathrm{mmol})$. The tube was evacuated and back-filled with nitrogen (this process was repeated three times). Then DMF $(4.0 \mathrm{~mL}), \mathbf{2 a}(84.0 \mathrm{mg}, 1.0 \mathrm{mmol})$ and triethylamine $(211.0 \mu \mathrm{L}, 1.5 \mathrm{mmol})$ were added consecutively via syringe. The resulting mixture was stirred at room temperature for $18 \mathrm{~h}$. The reaction mixture was quenched with $\mathrm{H}_{2} \mathrm{O}$, extracted with ethyl acetate $(60 \mathrm{~mL})$. The combined organic phase was washed with saturated brine $(3 \times 50 \mathrm{~mL})$, dried over anhydrous sodium sulfate, filtered and concentrated under reduced pressure. The crude mixture was purified by column chromatography on silica gel with hexane/ethyl acetate mixtures as eluent to give the corresponding product $7 \mathbf{d}(298.0 \mathrm{mg}, 84 \%)$ as yellow oil.

\section{Characterization of New Compounds}

\section{(Z)-4-Methyl-6-phenyl-8-(trimethylsilyl)oct-5-en-7-yn-2-one (4a)}<smiles>CC#C/C(=C\C(C)CC(C)=O)c1ccccc1</smiles>

Following Method A, $50.0 \mathrm{mg}$ of $\mathbf{4 a}$ (89\% yield) was obtained as light yellow oil. ${ }^{1} \mathbf{H}$ NMR $\left(600 \mathrm{MHz}, \mathrm{CDCl}_{3}\right) \delta 7.59(\mathrm{~d}, J=7.4$ $\mathrm{Hz}, 2 \mathrm{H}), 7.38-7.32(\mathrm{~m}, 2 \mathrm{H}), 7.29$ (t, $J=7.6 \mathrm{~Hz}, 1 \mathrm{H}), 6.27$ (d, $J$ $=9.4 \mathrm{~Hz}, 1 \mathrm{H}), 3.53-3.44(\mathrm{~m}, 1 \mathrm{H}), 2.62-2.52(\mathrm{~m}, 2 \mathrm{H}), 2.20(\mathrm{~s}$, $3 \mathrm{H}), 1.18(\mathrm{~d}, J=6.5 \mathrm{~Hz}, 3 \mathrm{H}), 0.29(\mathrm{~s}, 9 \mathrm{H}) ;{ }^{13} \mathbf{C} \mathbf{N M R}(151 \mathrm{MHz}$, $\left.\mathrm{CDCl}_{3}\right) \delta 207.9,143.1,137.5,128.5,127.9,126.1,123.2,101.9,101.4,50.7,32.4,30.0$, 20.1, 0.1; HRMS (ESI) $[\mathrm{M}+\mathrm{H}]^{+}$calcd for $\left[\mathrm{C}_{18} \mathrm{H}_{24} \mathrm{OSi}+\mathrm{H}\right]^{+} 285.1669$, found 285.1667 . 
<smiles>CC(=O)C#C/C(=C\C(C)CC(C)=O)c1ccc(N(C)C)cc1</smiles>

Following Method A, $45.8 \mathrm{mg}$ of $\mathbf{4 b}$ (70\% yield) was obtained as light yellow oil. ${ }^{1} \mathbf{H}$ NMR (600 MHz, $\left.\mathrm{CDCl}_{3}\right) \delta 7.45(\mathrm{~d}, J=8.9 \mathrm{~Hz}, 2 \mathrm{H}), 6.68(\mathrm{~d}, J=8.8 \mathrm{~Hz}$, 2H), $6.07(\mathrm{~d}, J=9.4 \mathrm{~Hz}, 1 \mathrm{H}), 3.48-3.41(\mathrm{~m}, 1 \mathrm{H}), 2.95$ $(\mathrm{s}, 6 \mathrm{H}), 2.58-2.44(\mathrm{~m}, 2 \mathrm{H}), 2.17(\mathrm{~s}, 3 \mathrm{H}), 1.13(\mathrm{~d}, J=$ $6.7 \mathrm{~Hz}, 3 \mathrm{H}), 0.25(\mathrm{~s}, 9 \mathrm{H}) ;{ }^{13} \mathbf{C} \mathbf{N M R}\left(151 \mathrm{MHz}, \mathrm{CDCl}_{3}\right)$ $\delta 208.3,150.4,139.2,126.9,125.7,122.8,112.3,102.6$, 100.6, 51.1, 40.7, 32.4, 29.9, 20.3, 0.2; HRMS (ESI) $[\mathrm{M}+\mathrm{H}]^{+}$calcd for $\left[\mathrm{C}_{20} \mathrm{H}_{29} \mathrm{NOSi}+\mathrm{H}\right]^{+}$328.2091, found 328.2087.

(Z)-4-Methyl-6-(m-tolyl)-8-(trimethylsilyl)oct-5-en-7-yn-2-one (4c)<smiles>CC#C/C(=C\C(C)CC(C)=O)c1ccc(C)cc1</smiles>

Following Method A, $50.7 \mathrm{mg}$ of $4 \mathbf{c}$ ( $85 \%$ yield) was obtained as light yellow oil. ${ }^{1} \mathbf{H}$ NMR $\left(400 \mathrm{MHz}, \mathrm{CDCl}_{3}\right) \delta$ $7.44(\mathrm{~d}, J=7.8 \mathrm{~Hz}, 2 \mathrm{H}), 7.13(\mathrm{~d}, J=7.8 \mathrm{~Hz}, 2 \mathrm{H}), 6.19$ (d, $J$ $=9.4 \mathrm{~Hz}, 1 \mathrm{H}), 3.44(\mathrm{dt}, J=14.6,7.0 \mathrm{~Hz}, 1 \mathrm{H}), 2.63-2.44$ (m, 2H), 2.34 (s, 3H), 2.17 (s, 3H), $1.14(\mathrm{~d}, J=6.6 \mathrm{~Hz}, 3 \mathrm{H})$, 0.25 (s, 9H). ${ }^{13} \mathbf{C ~ N M R}\left(101 \mathrm{MHz}, \mathrm{CDCl}_{3}\right) \delta 207.9,142.2,137.7,134.7,129.2,126.0$, 123.0, 102.1, 101.1, 50.8, 32.4, 30.0, 21.2, 20.2, 0.1; HRMS (ESI) $[\mathrm{M}+\mathrm{H}]^{+}$calcd for $\left[\mathrm{C}_{19} \mathrm{H}_{26} \mathrm{OSi}+\mathrm{H}\right]^{+} 299.1826$, found 299.1824.

(Z)-6-(4-Methoxyphenyl)-4-methyl-8-(trimethylsilyl)oct-5-en-7-yn-2-one (4d)<smiles>CC#CC(=CC(C)CC(C)=O)c1ccc(OC)cc1</smiles>

Following Method A, $47.1 \mathrm{mg}$ of $\mathbf{4 d}$ (75\% yield) was obtained as light yellow oil. ${ }^{\mathbf{1}} \mathbf{H}$ NMR $\left(600 \mathrm{MHz}, \mathrm{CDCl}_{3}\right)$ $\delta 7.39(\mathrm{~d}, J=8.8 \mathrm{~Hz}, 2 \mathrm{H}), 6.76(\mathrm{~d}, J=8.8 \mathrm{~Hz}, 2 \mathrm{H}), 6.03$ $(\mathrm{d}, J=9.4 \mathrm{~Hz}, 1 \mathrm{H}), 3.71(\mathrm{~s}, 3 \mathrm{H}), 3.37-3.30(\mathrm{~m}, 1 \mathrm{H}), 2.38$ $-2.50(\mathrm{~m}, 2 \mathrm{H}), 2.07(\mathrm{~s}, 3 \mathrm{H}), 1.04(\mathrm{~d}, J=6.7 \mathrm{~Hz}, 3 \mathrm{H}), 0.15$

(s, 9H); ${ }^{13} \mathbf{C}$ NMR (151 MHz, $\left.\mathrm{CDCl}_{3}\right) \delta 208.0,159.5,141.2,130.1,127.3,122.6,113.8$, 102.1, 101.1, 55.5, 50.9, 32.4, 30.0, 20.2, 0.1; HRMS (ESI) $[\mathrm{M}+\mathrm{Na}]^{+}$calcd for $\left[\mathrm{C}_{19} \mathrm{H}_{22} \mathrm{O}_{2} \mathrm{Si}+\mathrm{Na}\right]^{+} 337.1594$, found 337.1591. 
<smiles>CC(=O)CC(C)/C=C(\C#CC(C)(C)C)c1ccc(C(C)(C)C)cc1</smiles>

Following Method A, $50.4 \mathrm{mg}$ of $4 \mathrm{e}$ (74\% yield) was obtained as light yellow oil. ${ }^{1} \mathbf{H}$ NMR $\left(400 \mathrm{MHz}, \mathrm{CDCl}_{3}\right)$ $\delta 7.50(\mathrm{~d}, J=8.5 \mathrm{~Hz}, 2 \mathrm{H}), 7.36(\mathrm{~d}, J=8.5 \mathrm{~Hz}, 2 \mathrm{H}), 6.21(\mathrm{~d}$, $J=9.4 \mathrm{~Hz}, 1 \mathrm{H}), 3.51-3.40(\mathrm{~m}, 1 \mathrm{H}), 2.59-2.47(\mathrm{~m}, 2 \mathrm{H})$, $2.18(\mathrm{~s}, 3 \mathrm{H}), 1.32(\mathrm{~s}, 9 \mathrm{H}), 1.14(\mathrm{~d}, J=6.7 \mathrm{~Hz}, 3 \mathrm{H}), 0.26(\mathrm{~s}$, 9H); $\left.{ }^{13} \mathbf{C ~ N M R ~ ( 1 0 1 ~ M H z , ~} \mathrm{CDCl}_{3}\right) \delta 208.0,151.0,142.3,134.6,125.8,125.4,122.9$, 102.0, 101.1, 50.8, 34.6, 32.4, 31.4, 29.9, 20.2, 0.1; HRMS (ESI) $[\mathrm{M}+\mathrm{H}]^{+}$calcd for $\left[\mathrm{C}_{22} \mathrm{H}_{32} \mathrm{OSi}+\mathrm{H}\right]^{+}$341.2295, found 341.2292.

\section{(Z)-6-(4-Chlorophenyl)-4-methyl-8-(trimethylsilyl)oct-5-en-7-yn-2-one (4f)}<smiles>CC(=O)C#C/C(=C\C(C)CC(C)=O)c1ccc(Cl)cc1</smiles>

Following Method A, $52.2 \mathrm{mg}$ of $\mathbf{4 f}$ ( $82 \%$ yield) was obtained as light yellow oil. ${ }^{1} \mathbf{H}$ NMR $\left(400 \mathrm{MHz}, \mathrm{CDCl}_{3}\right) \delta$ $7.48(\mathrm{~d}, J=8.6 \mathrm{~Hz}, 2 \mathrm{H}), 7.28(\mathrm{~d}, J=8.6 \mathrm{~Hz}, 2 \mathrm{H}), 6.23(\mathrm{~d}, J$ $=9.4 \mathrm{~Hz}, 1 \mathrm{H}), 3.49-3.38(\mathrm{~m}, 1 \mathrm{H}), 2.60-2.49(\mathrm{~m}, 2 \mathrm{H}), 2.17$ $(\mathrm{s}, 3 \mathrm{H}), 1.15(\mathrm{~d}, J=6.7 \mathrm{~Hz}, 3 \mathrm{H}), 0.26(\mathrm{~s}, 9 \mathrm{H}) ;{ }^{13} \mathbf{C}$ NMR $(101$

$\left.\mathrm{MHz}, \mathrm{CDCl}_{3}\right) \delta 207.7,143.4,136.0,133.6,128.6,127.4,122.2,101.8,101.4,50.6,32.4$, 30.0, 20.0, 0.1; HRMS (ESI) $[\mathrm{M}+\mathrm{H}]^{+}$calcd for $\left[\mathrm{C}_{18} \mathrm{H}_{23} \mathrm{ClOSi}+\mathrm{Na}\right]^{+} 341.1099$, found 341.1097 .

(Z)-6-(4-Bromophenyl)-4-methyl-8-(trimethylsilyl)oct-5-en-7-yn-2-one (4g)<smiles>CC#C/C(=C\C(C)CC(C)=O)c1ccc(Br)cc1</smiles>
Following Method A, $52.0 \mathrm{mg}$ of $\mathbf{4 g}$ (71\% yield) was obtained as light yellow oil. ${ }^{1} \mathbf{H}$ NMR $\left(600 \mathrm{MHz}, \mathrm{CDCl}_{3}\right) \delta$ $7.40-7.44(\mathrm{~m}, 4 \mathrm{H}), 6.23(\mathrm{~d}, J=9.4 \mathrm{~Hz}, 1 \mathrm{H}), 3.47-3.40(\mathrm{~m}$, $1 \mathrm{H}), 2.58-2.50(\mathrm{~m}, 2 \mathrm{H}), 2.16(\mathrm{~s}, 3 \mathrm{H}), 1.14(\mathrm{~d}, J=6.7 \mathrm{~Hz}$, $3 \mathrm{H}), 0.25(\mathrm{~s}, 9 \mathrm{H}) ;{ }^{13} \mathrm{C}$ NMR $\left(151 \mathrm{MHz}, \mathrm{CDCl}_{3}\right) \delta$ 207.6, $143.5,136.4,131.4,127.7,122.2,121.7,101.8,101.2,50.5,32.3,30.0,19.9,0.1$; HRMS (ESI) $[\mathrm{M}+\mathrm{Na}]^{+}$calcd for $\left[\mathrm{C}_{18} \mathrm{H}_{23} \mathrm{BrOSi}+\mathrm{Na}\right]^{+}$385.0594, found 385.0592.

Methyl (Z)-4-(5-methyl-7-oxo-1-(trimethylsilyl)oct-3-en-1-yn-3-yl)benzoate (4h) 
(Me

Following Method A, $37.6 \mathrm{mg}$ of $\mathbf{4 h}$ (55\% yield) was obtained as light yellow oil. ${ }^{1} \mathbf{H}$ NMR $\left(400 \mathrm{MHz}, \mathrm{CDCl}_{3}\right)$ $\delta 8.00-7.96(\mathrm{~m}, 2 \mathrm{H}), 7.64-7.59(\mathrm{~m}, 2 \mathrm{H}), 6.36(\mathrm{~d}, J=$ $9.4 \mathrm{~Hz}, 1 \mathrm{H}), 3.91(\mathrm{~s}, 3 \mathrm{H}), 3.51-3.40(\mathrm{~m} \mathrm{1H}), 2.63-$ $2.52(\mathrm{~m}, 2 \mathrm{H}), 2.18(\mathrm{~s}, 3 \mathrm{H}), 1.16(\mathrm{~d}, J=6.7 \mathrm{~Hz}, 3 \mathrm{H}), 0.26$

(s, 9H); ${ }^{13} \mathbf{C}$ NMR (101 MHz, $\left.\mathrm{CDCl}_{3}\right) \delta 207.6,167.0,145.1,141.9,129.8,129.3,126.1$, 122.6, 102.2, 101.1, 52.2, 50.5, 32.5, 30.1, 20.0, 0.1; HRMS (ESI) $[\mathrm{M}+\mathrm{Na}]^{+}$calcd for $\left[\mathrm{C}_{18} \mathrm{H}_{23} \mathrm{FOSi}+\mathrm{Na}\right]^{+}$365.1543, found 365.1543 .

(Z)-6-(4-Chlorophenyl)-4-methyl-8-(trimethylsilyl)oct-5-en-7-yn-2-one (4i)<smiles>CC(=O)CC(C)/C=C(\C#CC#N)c1ccc(-c2ccccc2)cc1</smiles>
Following Method A, $50.4 \mathrm{mg}$ of $\mathbf{4 i}$ (70\% yield) was obtained as light yellow oil. ${ }^{1} \mathbf{H}$ NMR $\left(400 \mathrm{MHz}, \mathrm{CDCl}_{3}\right) \delta$ $7.51(\mathrm{~d}, J=7.4 \mathrm{~Hz}, 2 \mathrm{H}), 7.47(\mathrm{~d}, J=8.3 \mathrm{~Hz}, 2 \mathrm{H}), 7.44(\mathrm{~d}, J$ $=8.3 \mathrm{~Hz}, 2 \mathrm{H}), 7.31(\mathrm{t}, J=7.4 \mathrm{~Hz}, 2 \mathrm{H}), 7.23(\mathrm{t}, J=7.2 \mathrm{~Hz}$, $1 \mathrm{H}), 6.18(\mathrm{~d}, J=9.4 \mathrm{~Hz}, 1 \mathrm{H}), 3.42-3.26(\mathrm{~m}, 1 \mathrm{H}), 2.44(\mathrm{qd}$, $J=15.3,7.0 \mathrm{~Hz}, 2 \mathrm{H}), 2.06(\mathrm{~s}, 3 \mathrm{H}), 1.04$ (d, $J=6.7 \mathrm{~Hz}, 3 \mathrm{H}), 0.15$ (s, 9H).; ${ }^{13} \mathbf{C}$ NMR $\left(101 \mathrm{MHz}, \mathrm{CDCl}_{3}\right) \delta 207.8,142.9,140.6,140.5,136.3,128.8,127.3,127.03,127.00$, 126.4, 122.7, 101.7, 101.4, 50.6, 32.3, 29.9, 20.0, 0.1; HRMS (ESI) $[\mathrm{M}+\mathrm{H}]^{+}$calcd for $\left[\mathrm{C}_{24} \mathrm{H}_{28} \mathrm{ClOSi}+\mathrm{H}\right]^{+}$361.1982, found 361.1980.

\section{(Z)-6-(4-Fluorophenyl)-4-methyl-8-(trimethylsilyl)oct-5-en-7-yn-2-one (4j)}<smiles>CC#C/C(=C\C(C)CC(C)=O)c1ccc(F)cc1</smiles>

Following Method A, $50.1 \mathrm{mg}$ of $\mathbf{4 j}$ ( $83 \%$ yield) was obtained as light yellow oil. ${ }^{1} \mathbf{H}$ NMR $\left(400 \mathrm{MHz}, \mathrm{CDCl}_{3}\right) \delta 7.52(\mathrm{dd}, J$ $=8.6,5.4 \mathrm{~Hz}, 2 \mathrm{H}), 7.00(\mathrm{t}, J=8.6 \mathrm{~Hz}, 2 \mathrm{H}), 6.17(\mathrm{~d}, J=9.4$ $\mathrm{Hz}, 1 \mathrm{H}), 3.47-3.39(\mathrm{~m}, 1 \mathrm{H}), 2.59-2.48(\mathrm{~m}, 2 \mathrm{H}), 2.17(\mathrm{~s}$, $3 \mathrm{H}), 1.14(\mathrm{~d}, J=6.7 \mathrm{~Hz}, 3 \mathrm{H}), 0.25(\mathrm{~s}, 9 \mathrm{H}) ;{ }^{19}$ F NMR $(376$ $\left.\mathrm{MHz}, \mathrm{CDCl}_{3}\right) \delta-114.72 ;{ }^{13} \mathrm{C}$ NMR $\left(101 \mathrm{MHz}, \mathrm{CDCl}_{3}\right) \delta 207.8,162.6(\mathrm{~d}, J=246.9 \mathrm{~Hz})$, 142.8, $133.6(\mathrm{~d}, J=3.3 \mathrm{~Hz}), 127.8(\mathrm{~d}, J=8.1 \mathrm{~Hz}), 115.3$ (d, $J=21.6 \mathrm{~Hz}), 101.7,50.7$, 32.4, 30.0, 20.1, 0.1; HRMS (ESI) $[\mathrm{M}+\mathrm{H}]^{+}$calcd for $\left[\mathrm{C}_{18} \mathrm{H}_{23} \mathrm{FOSi}+\mathrm{H}\right]^{+}$303.1575, found 303.1576 .

(Z)-6-(3-Methoxyphenyl)-4-methyl-8-(trimethylsilyl)oct-5-en-7-yn-2-one (4k) 
TMS

Following Method A, $50.1 \mathrm{mg}$ of $\mathbf{4 k}$ (63\% yield) was obtained as light yellow oil. ${ }^{1} \mathbf{H}$ NMR $\left(400 \mathrm{MHz}, \mathrm{CDCl}_{3}\right)$ $\delta 7.49-7.41(\mathrm{~m}, 1 \mathrm{H}), 7.23(\mathrm{t}, J=7.9 \mathrm{~Hz}, 1 \mathrm{H}), 7.17-7.11$ (m, 1H), $6.81(\mathrm{ddd}, J=8.1,2.6,1.0 \mathrm{~Hz}, 1 \mathrm{H}), 6.25$ (d, $J=$ $9.4 \mathrm{~Hz}, 1 \mathrm{H}), 3.82(\mathrm{~s}, 3 \mathrm{H}), 3.51-3.41(\mathrm{~m}, 1 \mathrm{H}), 2.60-2.47$

(m, 2H), $2.17(\mathrm{~s}, 3 \mathrm{H}), 1.14(\mathrm{~d}, J=6.7 \mathrm{~Hz}, 3 \mathrm{H}), 0.25(\mathrm{~s}, 9 \mathrm{H}) ;{ }^{13} \mathbf{C}$ NMR (101 MHz, $\left.\mathrm{CDCl}_{3}\right) \delta 207.9,159.7,143.3,138.9,129.4,123.0,118.5,113.4,111.9,101.8,101.4$, 55.3, 50.7, 32.4, 30.0, 20.0, 0.1; HRMS (ESI) $[\mathrm{M}+\mathrm{H}]^{+}$calcd for $\left[\mathrm{C}_{19} \mathrm{H}_{26} \mathrm{O}_{2} \mathrm{Si}+\mathrm{H}\right]^{+}$ 315.1775, found 315.1773.

(Z)-4-Methyl-6-(m-tolyl)-8-(trimethylsilyl)oct-5-en-7-yn-2-one (4l)<smiles>CC(=O)CC(C)/C=C(\C#CC#N)c1cccc(C)c1</smiles>

Following Method A, $44.7 \mathrm{mg}$ of $4 \mathbf{l}$ (75\% yield) was obtained as light yellow oil. ${ }^{1} \mathbf{H}$ NMR $\left(400 \mathrm{MHz}, \mathrm{CDCl}_{3}\right) \delta 7.41-7.30(\mathrm{~m}$, 2H), $7.22(\mathrm{t}, J=7.9 \mathrm{~Hz}, 1 \mathrm{H}), 7.08$ (d, $J=7.5 \mathrm{~Hz}, 1 \mathrm{H}), 6.23$ (d, $J$ $=9.4 \mathrm{~Hz}, 1 \mathrm{H}), 3.51-3.40(\mathrm{~m}, 1 \mathrm{H}), 2.61-2.48(\mathrm{~m}, 2 \mathrm{H}), 2.36(\mathrm{~s}$, $3 \mathrm{H}), 2.18(\mathrm{~s}, 3 \mathrm{H}), 1.15$ (d, $J=6.7 \mathrm{~Hz}, 3 \mathrm{H}), 0.26(\mathrm{~s}, 9 \mathrm{H}) ;{ }^{13} \mathrm{C}$

NMR $\left(101 \mathrm{MHz}, \mathrm{CDCl}_{3}\right) \delta 208.0,142.9,138.0,137.4,128.6,128.3,126.8,123.3$, 123.2, 102.0, 101.2, 50.7, 32.4, 30.0, 21.6, 20.1, 0.1; HRMS (ESI) $[\mathrm{M}+\mathrm{Na}]^{+}$calcd for $\left[\mathrm{C}_{19} \mathrm{H}_{26} \mathrm{OSi}+\mathrm{Na}\right]^{+} 321.1645$, found 321.1642 .

\section{(Z)-6-(3-Fluorophenyl)-4-methyl-8-(trimethylsilyl)oct-5-en-7-yn-2-one (4m)}<smiles>CC#C/C(=C\C(C)CC(C)=O)c1cccc(F)c1</smiles>

Following Method A, $47.7 \mathrm{mg}$ of $\mathbf{4 m}$ (79\% yield) was obtained as light yellow oil. ${ }^{1} \mathbf{H}$ NMR $(400 \mathrm{MHz}$, Chloroformd) $\delta 7.40-7.20(\mathrm{~m}, 3 \mathrm{H}), 7.01-6.88(\mathrm{~m}, 1 \mathrm{H}), 6.27(\mathrm{~d}, J=9.4$ $\mathrm{Hz}, 1 \mathrm{H}), 3.52-3.34(\mathrm{~m}, 1 \mathrm{H}), 2.65-2.43(\mathrm{~m}, 2 \mathrm{H}), 2.18(\mathrm{~s}$, $3 \mathrm{H}), 1.15(\mathrm{~d}, J=6.7 \mathrm{~Hz}, 3 \mathrm{H}), 0.26(\mathrm{~s}, 9 \mathrm{H}) ;{ }^{19} \mathbf{F}$ NMR $(376$ $\left.\mathrm{MHz}, \mathrm{CDCl}_{3}\right) \delta-113.44 ;{ }^{13} \mathrm{C}$ NMR $(101 \mathrm{MHz}$, Chloroform- $d) \delta 207.7,163.0(\mathrm{~d}, J=$ $245.0 \mathrm{~Hz}), 144.0,139.8$ (d, $J=7.6 \mathrm{~Hz}), 129.9$ (d, $J=8.4 \mathrm{~Hz}), 122.3(\mathrm{~d}, J=2.6 \mathrm{~Hz})$, $121.7(\mathrm{~d}, J=2.8 \mathrm{~Hz}), 114.6(\mathrm{~d}, J=21.4 \mathrm{~Hz}), 113.2(\mathrm{~d}, J=22.9 \mathrm{~Hz}), 101.8,101.2,50.5$, 32.4, 30.0, 20.0; HRMS (ESI) $[\mathrm{M}+\mathrm{H}]^{+}$calcd for $\left[\mathrm{C}_{18} \mathrm{H}_{23} \mathrm{FO}+\mathrm{H}\right]^{+}$303.1575, found 303.1573 . 
(Z)-6-(3-Chlorophenyl)-4-methyl-8-(trimethylsilyl)oct-5-en-7-yn-2-one (4n)<smiles>CC#CC(=CC(C)CC(C)=O)c1cccc(Cl)c1</smiles>

Following Method A, $44.5 \mathrm{mg}$ of $4 \mathbf{n}$ (70\% yield) was obtained as light yellow oil. ${ }^{1} \mathbf{H}$ NMR $\left(400 \mathrm{MHz}, \mathrm{CDCl}_{3}\right) \delta$ $7.55(\mathrm{~s}, 1 \mathrm{H}), 7.46(\mathrm{dt}, J=6.9,1.9 \mathrm{~Hz}, 1 \mathrm{H}), 7.30-7.25(\mathrm{~m}$, 2H), $6.28(\mathrm{~d}, J=9.4 \mathrm{~Hz}, 1 \mathrm{H}), 3.51-3.40(\mathrm{~m}, 1 \mathrm{H}), 2.62-$ $2.52(\mathrm{~m}, 2 \mathrm{H}), 2.20(\mathrm{~s}, 3 \mathrm{H}), 1.17(\mathrm{~d}, J=6.7 \mathrm{~Hz}, 3 \mathrm{H}), 0.28(\mathrm{~s}$, 9H); ${ }^{13}$ C NMR (101 MHz, $\left.\mathrm{CDCl}_{3}\right) \delta 207.6,144.1,139.4,134.4,129.7,127.8,126.2$, 124.3, 122.1, 102.0, 101.2, 50.5, 32.4, 30.1, 20.0, 0.1; HRMS (ESI) $[\mathrm{M}+\mathrm{Na}]^{+}$calcd for $\left[\mathrm{C}_{18} \mathrm{H}_{23} \mathrm{ClOSi}+\mathrm{Na}\right]^{+}$341.1099, found 341.1097.

(Z)-6-(2-Methoxyphenyl)-4-methyl-8-(trimethylsilyl)oct-5-en-7-yn-2-one (40)<smiles>COc1ccccc1/C(C#CC#N)=C/C(C)CC(C)=O</smiles>

Following Method A, $45.2 \mathrm{mg}$ of $\mathbf{4 0}$ (72\% yield) was obtained as light yellow oil. ${ }^{1} \mathbf{H}$ NMR (400 MHz, $\left.\mathrm{CDCl}_{3}\right) \delta 7.23(\mathrm{t}, J=7.9$ $\mathrm{Hz}, 1 \mathrm{H}), 7.15$ (dt, $J=7.7,1.2 \mathrm{~Hz}, 1 \mathrm{H}), 7.13-7.12(\mathrm{~m}, 1 \mathrm{H}), 6.83$ $-6.80(\mathrm{~m}, 1 \mathrm{H}), 6.25(\mathrm{~d}, J=9.4 \mathrm{~Hz}, 1 \mathrm{H}), 3.82(\mathrm{~s}, 3 \mathrm{H}), 3.50-3.40$ $(\mathrm{m}, 1 \mathrm{H}), 2.61-2.47(\mathrm{~m}, 2 \mathrm{H}), 2.17(\mathrm{~s}, 3 \mathrm{H}), 1.14(\mathrm{~d}, J=6.7 \mathrm{~Hz}$, 3H), 0.25 (s, 9H); ${ }^{13} \mathbf{C}$ NMR (101 MHz, $\left.\mathrm{CDCl}_{3}\right) \delta 207.9,159.7,143.3,139.0,129.4$, 123.0, 118.5, 113.5, 111.9, 101.8, 101.4, 55.3, 50.7, 32.4, 30.0, 20.1, 0.1; HRMS (ESI) $[\mathrm{M}+\mathrm{H}]^{+}$calcd for $\left[\mathrm{C}_{19} \mathrm{H}_{26} \mathrm{O}_{2} \mathrm{Si}+\mathrm{H}\right]^{+} 315.1775$, found 315.1775 .

(Z)-6-(Benzo[d][1,3] dioxol-5-yl)-4-methyl-8-(trimethylsilyl)oct-5-en-7-yn-2-one (4p)

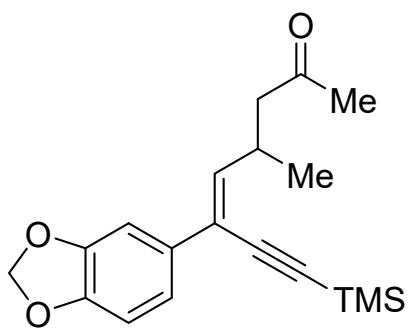

Following Method A, $44.6 \mathrm{mg}$ of $\mathbf{4 p}$ (68\% yield) was obtained as light yellow oil. ${ }^{1} \mathbf{H}$ NMR $\left(600 \mathrm{MHz} \mathrm{CDCl}_{3}\right) \delta$ 7.07 (dd, $J=8.1,1.7 \mathrm{~Hz}, 1 \mathrm{H}), 7.03(\mathrm{~s}, 1 \mathrm{H}), 6.76(\mathrm{~d}, J=8.1$ $\mathrm{Hz}, 1 \mathrm{H}), 6.10$ (d, $J=9.4 \mathrm{~Hz}, 1 \mathrm{H}), 5.94$ (s, 2H), 3.41 (ddd, $J$ $=14.1,9.4,6.8 \mathrm{~Hz}, 1 \mathrm{H}), 2.63-2.41(\mathrm{~m}, 2 \mathrm{H}), 2.16(\mathrm{~s}, 3 \mathrm{H})$,

$1.13(\mathrm{~d}, J=6.7 \mathrm{~Hz}, 3 \mathrm{H}), 0.25(\mathrm{~s}, 9 \mathrm{H}) ;{ }^{13} \mathbf{C} \mathbf{N M R}\left(151 \mathrm{MHz}, \mathrm{CDCl}_{3}\right) \delta 207.9,147.8$, $147.4,141.8,131.9,122.7,120.1,108.2,106.5,101.9,101.2,50.7,32.4,30.0,20.1,0.1$; HRMS (ESI) $[\mathrm{M}+\mathrm{H}]^{+}$calcd for $\left[\mathrm{C}_{19} \mathrm{H}_{24} \mathrm{OSi}+\mathrm{H}\right]^{+} 329.1567$, found 329.1567 .

(Z)-4-Methyl-6-(naphthalen-2-yl)-8-(trimethylsilyl)oct-5-en-7-yn-2-one (4q) 
<smiles>CC#C/C(=C\C(C)CC(C)=O)c1ccc2ccccc2c1</smiles>

Following Method A, $50.8 \mathrm{mg}$ of $\mathbf{4 q}$ (76\% yield) was obtained as light yellow oil. ${ }^{1} \mathbf{H}$ NMR $\left(400 \mathrm{MHz}, \mathrm{CDCl}_{3}\right) \delta$ $8.05(\mathrm{~s}, 1 \mathrm{H}), 7.87-7.81(\mathrm{~m}, 1 \mathrm{H}), 7.79(\mathrm{~d}, J=8.7 \mathrm{~Hz}, 2 \mathrm{H})$, $7.68(\mathrm{dd}, J=8.6,1.8 \mathrm{~Hz}, 1 \mathrm{H}), 7.51-7.40(\mathrm{~m}, 2 \mathrm{H}), 6.40(\mathrm{~d}$, $J=9.4 \mathrm{~Hz}, 1 \mathrm{H}), 3.53(\mathrm{dq}, J=9.3,6.7 \mathrm{~Hz}, 1 \mathrm{H}), 2.59$ (qd, $J$ $=15.3,7.0 \mathrm{~Hz}, 2 \mathrm{H}), 2.20(\mathrm{~s}, 3 \mathrm{H}), 1.20(\mathrm{~d}, J=6.7 \mathrm{~Hz}, 3 \mathrm{H}), 0.31(\mathrm{~s}, 9 \mathrm{H}) ;{ }^{13} \mathbf{C}$ NMR $(101$ $\left.\mathrm{MHz}, \mathrm{CDCl}_{3}\right) \delta 207.9,143.4,134.8,133.4,133.1,128.4,128.0,127.7,126.3,126.1$, $125.7,123.7,123.2,101.9,101.7,50.7,32.5,30.0,20.1,0.1$; HRMS (ESI) $[\mathrm{M}+\mathrm{H}]^{+}$ calcd for $\left[\mathrm{C}_{22} \mathrm{H}_{26} \mathrm{OSi}+\mathrm{H}\right]^{+} 335.1826$, found 335.1824 .

tert-Butyl (Z)-5-(5-methyl-7-oxo-1-(trimethylsilyl)oct-3-en-1-yn-3-yl)-1H-indole1-carboxylate (4r)<smiles>CC(=O)C#C/C(=C\C(C)CC(C)=O)c1ccc2[nH]ccc2c1</smiles>

Boc
Following Method A, $52.4 \mathrm{mg}$ of $4 \mathbf{r}$ (62\% yield) was obtained as light yellow oil. ${ }^{1} \mathbf{H}$ NMR $\left(600 \mathrm{MHz}, \mathrm{CDCl}_{3}\right)$ $\delta 8.06(\mathrm{~s}, 1 \mathrm{H}), 7.75(\mathrm{~d}, J=1.6 \mathrm{~Hz}, 1 \mathrm{H}), 7.58(\mathrm{~d}, J=2.4 \mathrm{~Hz}$, $1 \mathrm{H}), 7.52(\mathrm{dd}, J=8.7,1.8 \mathrm{~Hz}, 1 \mathrm{H}), 6.56(\mathrm{~d}, J=3.6 \mathrm{~Hz}$, $1 \mathrm{H}), 6.26(\mathrm{~d}, J=9.4 \mathrm{~Hz}, 1 \mathrm{H}), 3.52-3.44(\mathrm{~m}, 1 \mathrm{H}), 2.63-$ $2.49(\mathrm{~m}, 2 \mathrm{H}), 2.19(\mathrm{~s}, 3 \mathrm{H}), 1.67(\mathrm{~s}, 9 \mathrm{H}), 1.17(\mathrm{~d}, J=6.7 \mathrm{~Hz}, 3 \mathrm{H}), 0.28(\mathrm{~s}, 9 \mathrm{H}) ;{ }^{13} \mathrm{C}$ NMR $\left(151 \mathrm{MHz}, \mathrm{CDCl}_{3}\right) \delta 208.0,149.8,142.4,134.9,132.3,130.8,126.5,123.3$, $122.5,118.8,115.0,107.6,102.4,101.2,83.9,50.8,32.5,30.0,28.3,20.2,0.1$; HRMS (ESI) $[\mathrm{M}+\mathrm{H}]^{+}$calcd for $\left[\mathrm{C}_{25} \mathrm{H}_{33} \mathrm{NO}_{3} \mathrm{Si}+\mathrm{H}\right]^{+}$424.2302, found 424.2302 .

\section{(E)-4-Methyl-6-(thiophen-2-yl)-8-(trimethylsilyl)oct-5-en-7-yn-2-one (4s)}

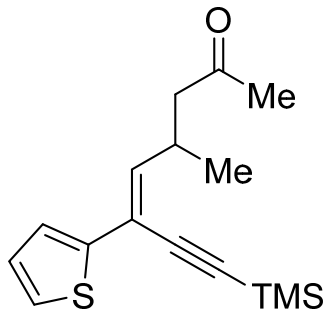

Following Method A, $39.4 \mathrm{mg}$ of $\mathbf{4 s}$ (68\% yield) was obtained as light yellow oil. ${ }^{1} \mathbf{H}$ NMR $\left(600 \mathrm{MHz}, \mathrm{CDCl}_{3}\right) \delta 7.18(\mathrm{dd}, J=3.6$, $0.8 \mathrm{~Hz}, 1 \mathrm{H}), 7.14(\mathrm{dd}, J=5.0,0.8 \mathrm{~Hz}, 1 \mathrm{H}), 6.96(\mathrm{dd}, J=5.0,3.6$ $\mathrm{Hz}, 1 \mathrm{H}), 6.14(\mathrm{~d}, J=9.4 \mathrm{~Hz}, 1 \mathrm{H}), 3.43-3.36(\mathrm{~m}, 1 \mathrm{H}), 2.57-$ $2.46(\mathrm{~m}, 2 \mathrm{H}), 2.17(\mathrm{~s}, 3 \mathrm{H}), 1.13(\mathrm{~d}, J=6.7 \mathrm{~Hz}, 3 \mathrm{H}), 0.26(\mathrm{~s}, 9 \mathrm{H})$;

${ }^{13}$ C NMR $\left(151 \mathrm{MHz}, \mathrm{CDCl}_{3}\right) \delta 207.7,142.4,141.0,127.4,125.1,124.5,117.6,100.8$, 100.6, 50.6, 32.1, 30.1, 20.1, 0.03; HRMS (ESI) $[\mathrm{M}+\mathrm{H}]^{+}$calcd for $\left[\mathrm{C}_{16} \mathrm{H}_{22} \mathrm{OSSi}+\mathrm{H}\right]^{+}$ 291.1233, found 291.1232. 
(Z)-5-Methyl-7-phenyl-9-(trimethylsilyl)non-6-en-8-yn-3-one (5a)

Following Method A, $45.9 \mathrm{mg}$ of $\mathbf{5 a}(77 \%$ yield $)$ was obtained as
light yellow oil. ${ }^{1} \mathbf{H}$ NMR $\left(400 \mathrm{MHz}, \mathrm{CDCl}_{3}\right) \delta 7.49-7.41(\mathrm{~m}, 2 \mathrm{H})$, $0.95(\mathrm{t}, J=7.3 \mathrm{~Hz}, 3 \mathrm{H}), 0.15(\mathrm{~s}, 9 \mathrm{H}) ;{ }^{13} \mathrm{C}$ NMR (101 MHz, $\left.\mathrm{CDCl}_{3}\right) \delta$ 210.4, 143.4, 137.6, 128.5, 127.8, 126.1, 123.0, 101.9, 101.3, 49.4, 36.0, 32.5, 20.2 , 7.9, 0.1; HRMS (ESI) $[\mathrm{M}+\mathrm{Na}]^{+}$calcd for $\left[\mathrm{C}_{19} \mathrm{H}_{26} \mathrm{OSi}+\mathrm{Na}\right]^{+} 321.1645$, found 321.1644 .

(Z)-6-Phenyl-4-propyl-8-(trimethylsilyl)oct-5-en-7-yn-2-one (5b)<smiles>CCCC(/C=C(\CC(C)=O)c1ccccc1)CC(C)=O</smiles>

Following Method A, $46.8 \mathrm{mg}$ of $\mathbf{5 b}$ (75\% yield) was obtained as light yellow oil. ${ }^{1} \mathbf{H}$ NMR $\left(600 \mathrm{MHz}, \mathrm{CDCl}_{3}\right) \delta 7.49(\mathrm{~d}, J=7.9 \mathrm{~Hz}$, 2H), $7.25(\mathrm{t}, J=7.5 \mathrm{~Hz}, 2 \mathrm{H}), 7.20-7.14(\mathrm{~m}, 1 \mathrm{H}), 6.12(\mathrm{~d}, J=9.9$ $\mathrm{Hz}, 1 \mathrm{H}), 3.33$ (h, $J=7.6 \mathrm{~Hz}, 1 \mathrm{H}), 2.46$ (ddd, $J=50.7,14.9,7.0 \mathrm{~Hz}$, 2H), $2.10(\mathrm{~s}, 3 \mathrm{H}), 1.40-1.24(\mathrm{~m}, 4 \mathrm{H}), 0.86(\mathrm{t}, J=7.1 \mathrm{~Hz}, 3 \mathrm{H}), 0.18(\mathrm{~s}, 9 \mathrm{H}) ;{ }^{13} \mathbf{C}$ NMR $\left(151 \mathrm{MHz}, \mathrm{CDCl}_{3}\right) \delta 208.1,142.1,137.5,128.5,127.9,126.2,124.3,102.3,100.9,49.7$, $37.5,37.4,29.9,20.6,14.2,0.1$; HRMS (ESI) $[\mathrm{M}+\mathrm{Na}]^{+}$calcd for $\left[\mathrm{C}_{20} \mathrm{H}_{28} \mathrm{OSi}+\mathrm{Na}\right]^{+}$ 335.1802 , found 335.1799 .

\section{(Z)-6-Phenyl-8-(trimethylsilyl)oct-5-en-7-yn-2-one (5c)}

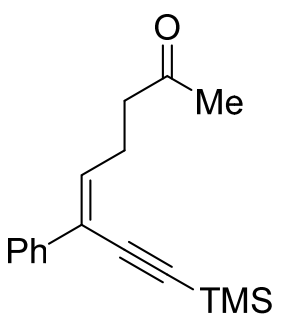

Following Method A, $45.9 \mathrm{mg}$ of $\mathbf{5 c}$ (85\% yield) was obtained as light yellow oil. ${ }^{1} \mathbf{H}$ NMR $\left(400 \mathrm{MHz}, \mathrm{CDCl}_{3}\right) \delta 7.45(\mathrm{~d}, J=7.5 \mathrm{~Hz}$, 2H), $7.22(\mathrm{dd}, J=7.5,6.6 \mathrm{~Hz}, 2 \mathrm{H}), 7.18-7.13(\mathrm{~m}, 1 \mathrm{H}), 6.33$ (t, $J=$

TMS $7.5 \mathrm{~Hz}, 1 \mathrm{H}), 2.69-2.61(\mathrm{~m}, 2 \mathrm{H}), 2.55(\mathrm{t}, J=6.9 \mathrm{~Hz}, 2 \mathrm{H}), 2.08$ (s, 3H), 0.15 (s, 9H); ${ }^{13} \mathbf{C}$ NMR (101 MHz, $\left.\mathrm{CDCl}_{3}\right) \delta 208.1,137.6,137.4,128.5,127.8$, 126.1, 101.9, 101.4, 42.8, 29.9, 25.7, 0.2; HRMS (ESI) $[\mathrm{M}+\mathrm{Na}]^{+}$calcd for $\left[\mathrm{C}_{17} \mathrm{H}_{23} \mathrm{OSi}+\mathrm{Na}\right]^{+}$271.1513, found 271.1509.

(Z)-7-phenyl-9-(trimethylsilyl)non-6-en-8-yn-3-one (5d) 


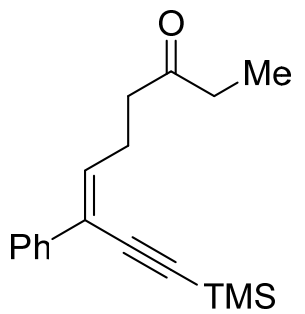

Following Method A, $46.6 \mathrm{mg}$ of $\mathbf{5 d}$ (82\% yield) was obtained as light yellow oil. ${ }^{1} \mathbf{H}$ NMR $\left(600 \mathrm{MHz}, \mathrm{CDCl}_{3}\right) \delta 7.58(\mathrm{~d}, J=7.6 \mathrm{~Hz}$, 2H), $7.39-7.31(\mathrm{~m}, 2 \mathrm{H}), 7.28(\mathrm{t}, J=7.1 \mathrm{~Hz}, 1 \mathrm{H}), 6.47(\mathrm{t}, J=7.3$

$\mathrm{Hz}, 1 \mathrm{H}), 2.78$ (q, $J=7.3 \mathrm{~Hz}, 2 \mathrm{H}), 2.65$ (t, $J=7.2 \mathrm{~Hz}, 2 \mathrm{H}), 2.49$ (q, $J=7.3 \mathrm{~Hz}, 2 \mathrm{H}), 1.10(\mathrm{t}, J=7.3 \mathrm{~Hz}, 3 \mathrm{H}), 0.28(\mathrm{~s}, 9 \mathrm{H}) ;{ }^{13} \mathbf{C ~ N M R}\left(101 \mathrm{MHz}, \mathrm{CDCl}_{3}\right) \delta$ $210.3,142.2,137.8,131.6,128.41,128.38,127.7,126.1,123.3,122.9,95.8,86.3,49.3$, 36.2, 32.4, 20.3, 7.8, 0.2; HRMS (ESI) $[\mathrm{M}+\mathrm{H}]^{+}$calcd for $\left[\mathrm{C}_{18} \mathrm{H}_{24} \mathrm{OSi}+\mathrm{H}\right]^{+}$285.1669, found 285.1667.

(Z)-3-(2-Phenyl-4-(trimethylsilyl)but-1-en-3-yn-1-yl)cyclopentan-1-one (5e)

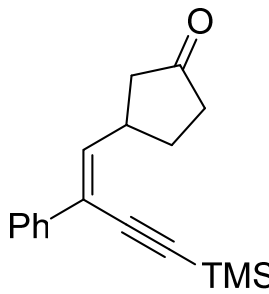

Following Method A, $31.0 \mathrm{mg}$ of $\mathbf{5 e}$ (55\% yield) was obtained as light yellow oil. ${ }^{1} \mathbf{H}$ NMR $\left(400 \mathrm{MHz}, \mathrm{CDCl}_{3}\right) \delta 7.60(\mathrm{~d}, J=7.3 \mathrm{~Hz}$, 2H), $7.37-7.32(\mathrm{~m}, 2 \mathrm{H}), 7.31-7.24(\mathrm{~m}, 1 \mathrm{H}), 6.28(\mathrm{~d}, J=9.0 \mathrm{~Hz}$,

TMS $1 \mathrm{H}), 3.34-3.23(\mathrm{~m}, 1 \mathrm{H}), 2.68-2.53(\mathrm{~m}, 1 \mathrm{H}), 2.48-2.21(\mathrm{~m}, 3 \mathrm{H})$, $2.08(\mathrm{ddd}, J=18.4,10.5,1.3 \mathrm{~Hz}, 1 \mathrm{H}), 1.90-1.71(\mathrm{~m}, 1 \mathrm{H}), 0.26(\mathrm{~s}, 9 \mathrm{H}) ;{ }^{13} \mathbf{C} \mathbf{~ N M R}$ $\left(151 \mathrm{MHz}, \mathrm{CDCl}_{3}\right) \delta 218.6,140.5,137.3,128.5,128.0,126.1,124.6,101.8,101.6,44.8$, 39.1, 38.4, 29.6, 0.1; HRMS (ESI) $[\mathrm{M}+\mathrm{H}]^{+}$calcd for $\left[\mathrm{C}_{18} \mathrm{H}_{23} \mathrm{OSi}+\mathrm{H}\right]^{+} 283.1513$, found 283.1516 .

(Z)-3-(2-Phenyl-4-(trimethylsilyl)but-1-en-3-yn-1-yl)cyclohexan-1-one (5f)<smiles>CC#C/C(=C\C1CCCC(=O)C1)c1ccccc1</smiles>

Following Method A, $41.4 \mathrm{mg}$ of $\mathbf{5 f}(70 \%$ yield) was obtained as light yellow oil. ${ }^{1} \mathbf{H}$ NMR (400 MHz, $\left.\mathrm{CDCl}_{3}\right): \delta 7.60(\mathrm{~d}, J=7.3 \mathrm{~Hz}$, 2H), $7.39-7.34(\mathrm{~m}, 2 \mathrm{H}), 7.33-7.27(\mathrm{~m}, 1 \mathrm{H}), 6.28(\mathrm{~d}, J=9.0 \mathrm{~Hz}$,

$1 \mathrm{H}), 3.34-3.23(\mathrm{~m}, 1 \mathrm{H}), 2.61-2.44(\mathrm{~m}, 2 \mathrm{H}), 2.39-2.27(\mathrm{~m}, 2 \mathrm{H})$, $2.16-2.03(\mathrm{~m}, 2 \mathrm{H}), 1.83-1.65(\mathrm{~m}, 2 \mathrm{H}), 0.28(\mathrm{~s}, 9 \mathrm{H}) ;{ }^{13} \mathbf{C ~ N M R}\left(101 \mathrm{MHz}, \mathrm{CDCl}_{3}\right) \delta$ 210.6, 140.6, 137.3, 128.5, 128.0, 126.1, 123.8, 101.8, 101.5, 46.8, 41.4, 41.1, 30.9, 25.4, 0.1; HRMS (ESI) $[\mathrm{M}+\mathrm{H}]^{+}$calcd for $\left[\mathrm{C}_{19} \mathrm{H}_{24} \mathrm{OSi}+\mathrm{H}\right]^{+} 297.1669$, found 297.1667. (Z)-3-(2-Phenyl-4-(trimethylsilyl)but-1-en-3-yn-1-yl)cycloheptan-1-one (5g) 
<smiles>C#C/C(=C\C1CCCCC(=O)C1)c1ccccc1</smiles>

Following Method A, $40.3 \mathrm{mg}$ of $\mathbf{5 g}$ (65\% yield) was obtained as light yellow oil. ${ }^{1} \mathbf{H}$ NMR (600 MHz, $\left.\mathrm{CDCl}_{3}\right) \delta 7.57-7.53(\mathrm{~m}, 1 \mathrm{H})$, $7.33(\mathrm{t}, J=7.6 \mathrm{~Hz}, 2 \mathrm{H}), 7.30-7.25(\mathrm{~m}, 1 \mathrm{H}), 6.26(\mathrm{~d}, J=9.2 \mathrm{~Hz}$, $1 \mathrm{H}), 3.23-3.17(\mathrm{~m}, 1 \mathrm{H}), 2.68-2.67(\mathrm{~m}, 1 \mathrm{H}), 2.61-2.53(\mathrm{~m} \mathrm{3H})$, $2.03-1.94(\mathrm{~m}, 3 \mathrm{H}), 1.66-1.61(\mathrm{~m}, 1 \mathrm{H}), 1.59-1.50(\mathrm{~m}, 2 \mathrm{H}), 0.26$

(s, 9H); ${ }^{13} \mathrm{C}$ NMR $\left(151 \mathrm{MHz}, \mathrm{CDCl}_{3}\right) \delta 213.2,142.3,137.4,128.5,127.9,126.2,123.1$, 101.9, 101.7, 49.2, 44.2, 38.1, 36.7, 29.2, 24.5, 0.2; HRMS (ESI) $[\mathrm{M}+\mathrm{H}]^{+}$calcd for $\left[\mathrm{C}_{20} \mathrm{H}_{26} \mathrm{OSi}+\mathrm{H}\right]^{+} 311.1826$, found 311.1825.

\section{(Z)-4,9,9-Trimethyl-6-phenyldec-5-en-7-yn-2-one (6a)}

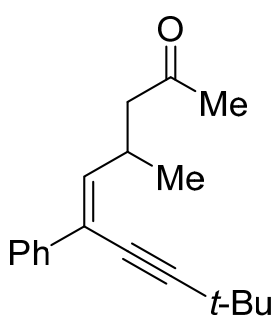

Following Method A, $37.5 \mathrm{mg}$ of $\mathbf{6 a}$ (70\% yield) was obtained as light yellow oil. ${ }^{1} \mathbf{H}$ NMR (400 MHz, $\left.\mathrm{CDCl}_{3}\right) \delta 7.57-7.54(\mathrm{~m}, 2 \mathrm{H})$, $7.34-7.29(\mathrm{~m}, 2 \mathrm{H}), 7.26-7.22(\mathrm{~m}, 1 \mathrm{H}), 6.13(\mathrm{~d}, J=9.3 \mathrm{~Hz}, 1 \mathrm{H})$, $3.47-7.36(\mathrm{~m}, 1 \mathrm{H}), 3.57-3.27(\mathrm{~m}, 1 \mathrm{H}), 2.60-2.46(\mathrm{~m}, 2 \mathrm{H}), 2.17$ (s, 3H), $1.34(\mathrm{~s}, 9 \mathrm{H}), 1.14(\mathrm{~d}, J=6.7 \mathrm{~Hz}, 3 \mathrm{H}) ;{ }^{13} \mathbf{C}$ NMR $(101 \mathrm{MHz}$, $\left.\mathrm{CDCl}_{3}\right) \delta 208.2,140.3,138.4,128.4,127.6,126.1,123.4,105.5,76.0,50.9,32.3,31.2$, 30.1, 28.5, 20.2; HRMS (ESI) $[\mathrm{M}+\mathrm{H}]^{+}$calcd for $\left[\mathrm{C}_{19} \mathrm{H}_{24} \mathrm{O}+\mathrm{H}\right]^{+}$269.1894, found 269.1898.

\section{(Z)-8-(tert-Butyldimethylsilyl)-4-methyl-6-phenyloct-5-en-7-yn-2-one (6b)}<smiles>CC(=O)CC(C)/C=C(\C#CC(C)(C)C)c1ccccc1</smiles>

Following Method A, $48.9 \mathrm{mg}$ of $\mathbf{6 b}$ (75\% yield) was obtained as light yellow oil. ${ }^{1} \mathbf{H}$ NMR $\left(400 \mathrm{MHz}, \mathrm{CDCl}_{3}\right) \delta 7.50(\mathrm{~d}, J=8.3 \mathrm{~Hz}$, 2H), $7.28-7.22(\mathrm{~m}, 2 \mathrm{H}), 7.21-7.16(\mathrm{~m}, 1 \mathrm{H}), 6.18(\mathrm{~d}, J=9.4 \mathrm{~Hz}$, 1H), $3.40(\mathrm{dtd}, J=14.2,6.7,2.7 \mathrm{~Hz}, 1 \mathrm{H}), 2.47(\mathrm{qd}, J=15.3,7.0 \mathrm{~Hz}$, 2H), 2.09 (s, 3H), 1.08 (d, J=6.7 Hz, 3H), 0.93 (s, 9H), 0.12 (s, 6H);

${ }^{13}$ C NMR $\left(101 \mathrm{MHz}, \mathrm{CDCl}_{3}\right) \delta 207.8,143.0,137.5,128.5,127.8,126.1,123.3,102.4$, 99.7, 50.7, 32.5, 30.0, 26.3, 20.1, 16.8, -4.4; HRMS (ESI) $[\mathrm{M}+\mathrm{H}]^{+}$calcd for $\left[\mathrm{C}_{21} \mathrm{H}_{30} \mathrm{OSi}+\mathrm{H}\right]^{+}$327.2139, found 327.2138.

(Z)-4-Methyl-6-phenyldodec-5-en-7-yn-2-one (6c) 
<smiles>CCCCC#C/C(=C/C(C)CC(C)=O)c1ccccc1</smiles>

Following Method A, $32.2 \mathrm{mg}$ of $\mathbf{6 c}(60 \%$ yield) was obtained as light yellow oil. ${ }^{1} \mathbf{H}$ NMR (400 MHz, $\left.\mathrm{CDCl}_{3}\right) \delta 7.48-7.50(\mathrm{~m}, 2 \mathrm{H})$, $7.26-7.22(\mathrm{~m}, 2 \mathrm{H}), 7.19-7.14(\mathrm{~m}, 1 \mathrm{H}), 6.05(\mathrm{~d}, J=9.4 \mathrm{~Hz}, 1 \mathrm{H})$, $3.30-3.41(\mathrm{~m}, 1 \mathrm{H}), 2.52-2.42(\mathrm{~m}, 2 \mathrm{H}), 2.39(\mathrm{t}, J=7.0 \mathrm{~Hz}, 2 \mathrm{H})$, $2.09(\mathrm{~s}, 3 \mathrm{H}), 1.57-1.50(\mathrm{~m}, 2 \mathrm{H}), 1.46-1.37(\mathrm{~m}, 2 \mathrm{H}), 1.06(\mathrm{~d}, J=$

$6.7 \mathrm{~Hz}, 3 \mathrm{H}), 0.88(\mathrm{t}, J=7.3 \mathrm{~Hz}, 3 \mathrm{H}) ;{ }^{13} \mathbf{C} \mathbf{~ N M R}\left(101 \mathrm{MHz}, \mathrm{CDCl}_{3}\right) \delta 208.1,140.5$, $138.5,128.4,127.6,126.2,123.4,107.0,97.2,50.8,32.3,31.1,30.1,22.2,20.3,19.4$, 13.7; HRMS (ESI) $[\mathrm{M}+\mathrm{H}]^{+}$calcd for $\left[\mathrm{C}_{19} \mathrm{H}_{24} \mathrm{O}+\mathrm{H}\right]^{+}$260.1900, found 269.1898 .

\section{(Z)-4-Methyl-6-phenyl-8-(triethylsilyl)oct-5-en-7-yn-2-one (6d)}<smiles>CC(=O)CC(C)/C=C(\C#CC(F)(F)F)c1ccccc1</smiles>

Following Method A, $46.9 \mathrm{mg}$ of $\mathbf{6 d}$ (72\% yield) was obtained as light yellow oil. ${ }^{1} \mathbf{H}$ NMR (400 MHz, $\left.\mathrm{CDCl}_{3}\right) \delta 7.55-7.47(\mathrm{~m}, 2 \mathrm{H})$, $7.27-7.22(\mathrm{~m}, 2 \mathrm{H}), 7.21-7.17(\mathrm{~m}, 1 \mathrm{H}), 6.18(\mathrm{~d}, J=9.4 \mathrm{~Hz}, 1 \mathrm{H})$, $3.49-3.35(\mathrm{~m}, 1 \mathrm{H}), 2.56-2.38(\mathrm{~m}, 2 \mathrm{H}), 2.09(\mathrm{~s}, 3 \mathrm{H}), 1.08(\mathrm{~d}, J=$ $6.7 \mathrm{~Hz}, 3 \mathrm{H}), 0.98$ (t, $J=7.9 \mathrm{~Hz}, 9 \mathrm{H}), 0.61$ (q, $J=7.9 \mathrm{~Hz}, 6 \mathrm{H}) ;{ }^{13} \mathbf{C}$ NMR (101 MHz, $\left.\mathrm{CDCl}_{3}\right) \delta 207.8,142.9,137.5,128.4,127.8,126.1,123.3,102.9,98.9,50.7,32.5,29.9$, 20.1, 7.7, 4.6; HRMS (ESI) $[\mathrm{M}+\mathrm{Na}]^{+}$calcd for $\left[\mathrm{C}_{21} \mathrm{H}_{30} \mathrm{OSi}+\mathrm{Na}\right]^{+} 349.1958$, found 349.1954.

\section{(Z)-6-(4-Chlorophenyl)-4-methyl-8-phenyloct-5-en-7-yn-2-one (6e)}<smiles>CC(=O)CC(C)/C=C(\C#Cc1ccccc1)c1ccc(Cl)cc1</smiles>

Following Method A, $60.5 \mathrm{mg}$ of $6 \mathrm{e}$ (94\% yield) was obtained as light yellow oil. ${ }^{1} \mathbf{H} \mathbf{~ N M R}\left(600 \mathrm{MHz}, \mathrm{CDCl}_{3}\right) \delta 7.60-7.56$ (m, 2H), $7.55-7.50(\mathrm{~m}, 2 \mathrm{H}), 7.38-7.34(\mathrm{~m}, 3 \mathrm{H}), 7.34-7.29$ $(\mathrm{m}, 2 \mathrm{H}), 6.26(\mathrm{~d}, J=9.5 \mathrm{~Hz}, 1 \mathrm{H}), 3.60-3.48(\mathrm{~m}, 1 \mathrm{H}), 2.65-$ $2.53(\mathrm{~m}, 2 \mathrm{H}), 2.18(\mathrm{~s}, 3 \mathrm{H}), 1.20(\mathrm{~d}, J=6.7 \mathrm{~Hz}, 3 \mathrm{H}) ;{ }^{13} \mathbf{C} \mathbf{N M R}$

$\left(151 \mathrm{MHz}, \mathrm{CDCl}_{3}\right) \delta 207.6,142.3,136.3,133.6,131.6,128.6,128.54,128.50,127.4$, 123.0, 122.0, 96.1, 85.8, 50.5, 32.4, 30.2, 20.2; HRMS (ESI) $[\mathrm{M}+\mathrm{H}]^{+}$calcd for $\left[\mathrm{C}_{21} \mathrm{H}_{19} \mathrm{ClO}+\mathrm{H}\right]^{+}$323.1197, found 323.1193.

(Z)-6-(4-Bromophenyl)-4-methyl-8-phenyloct-5-en-7-yn-2-one (6f) 
<smiles>CC(=O)CC(C)/C=C(\C#Cc1ccccc1)c1ccc(Br)cc1</smiles>

Following Method A, $67.3 \mathrm{mg}$ of $\mathbf{6 f}$ (92\% yield) was obtained as light yellow oil. ${ }^{1} \mathbf{H}$ NMR $\left(600 \mathrm{MHz}, \mathrm{CDCl}_{3}\right) \delta 7.54-7.49$ (m, 4H), $7.47(\mathrm{~d}, J=8.7 \mathrm{~Hz}, 2 \mathrm{H}), 7.39-7.31(\mathrm{~m}, 3 \mathrm{H}), 6.27$ (d, $J=9.5 \mathrm{~Hz}, 1 \mathrm{H}), 3.53(\mathrm{dq}, J=9.2,6.8 \mathrm{~Hz}, 1 \mathrm{H}), 2.60(\mathrm{qd}, J=$ 15.7, $6.9 \mathrm{~Hz}, 2 \mathrm{H}), 2.18(\mathrm{~s}, 3 \mathrm{H}), 1.19(\mathrm{~d}, J=6.7 \mathrm{~Hz}, 3 \mathrm{H}) ;{ }^{13} \mathbf{C}$

NMR $\left(151 \mathrm{MHz}, \mathrm{CDCl}_{3}\right) \delta 207.6,142.4,136.8,131.6,131.5,128.6,128.5,127.7$, 123.0, 122.1, 121.7, 96.2, 85.7, 50.5, 32.4, 30.2, 20.1; HRMS (ESI) $[\mathrm{M}+\mathrm{H}]^{+}$calcd for $\left[\mathrm{C}_{21} \mathrm{H}_{19} \mathrm{BrO}+\mathrm{H}\right]^{+}$367.0692, found 367.0694.

(Z)-6-(3-Fluorophenyl)-4-methyl-8-phenyloct-5-en-7-yn-2-one (6g)

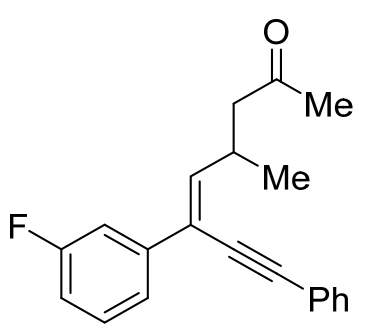

Following Method A, $46.8 \mathrm{mg}$ of $\mathbf{6 g}$ (76\% yield) was obtained as light yellow oil. ${ }^{1} \mathbf{H}$ NMR $\left(600 \mathrm{MHz}, \mathrm{CDCl}_{3}\right) \delta 7.55-7.52$ (m, 2H), $7.43(\mathrm{dt}, J=7.8,1.3 \mathrm{~Hz}, 1 \mathrm{H}), 7.39-7.33(\mathrm{~m}, 4 \mathrm{H}), 7.31$ $(\mathrm{td}, J=8.0,6.0 \mathrm{~Hz}, 1 \mathrm{H}), 6.98(\mathrm{tdd}, J=8.3,2.6,0.9 \mathrm{~Hz}, 1 \mathrm{H})$, $6.30(\mathrm{~d}, J=9.4 \mathrm{~Hz}, 1 \mathrm{H}), 3.55(\mathrm{dq}, J=9.3,6.8 \mathrm{~Hz}, 1 \mathrm{H}), 2.60(\mathrm{qd}$, $J=15.7,6.9 \mathrm{~Hz}, 2 \mathrm{H}), 2.19$ (s, 3H), $1.20(\mathrm{~d}, J=6.7 \mathrm{~Hz}, 3 \mathrm{H}) ;{ }^{19}$ F NMR $(376 \mathrm{MHz}$, $\left.\mathrm{CDCl}_{3}\right) \delta-113.33(\mathrm{~s}, 1 \mathrm{~F}) ;{ }^{13} \mathrm{C}$ NMR $\left(151 \mathrm{MHz}, \mathrm{CDCl}_{3}\right) \delta 207.7,163.1(\mathrm{~d}, J=245.2$ Hz), 143.0, 140.2 (d, $J=7.6 \mathrm{~Hz}), 131.7,129.9$ (d, $J=8.3 \mathrm{~Hz}), 128.7,128.6,123.1$, $122.2(\mathrm{~d}, J=2.7 \mathrm{~Hz}), 121.8(\mathrm{~d}, J=2.7 \mathrm{~Hz}), 114.7(\mathrm{~d}, J=21.3 \mathrm{~Hz}), 113.2(\mathrm{~d}, J=22.9$ $\mathrm{Hz}), 96.2,85.8,50.6,32.4,30.3,20.2$; HRMS (ESI) $[\mathrm{M}+\mathrm{H}]^{+}$calcd for $\left[\mathrm{C}_{21} \mathrm{H}_{19} \mathrm{FO}+\mathrm{H}\right]^{+}$ 307.1493, found 307.1483.

(Z)-5-Methyl-7,9-diphenylnon-6-en-8-yn-3-one (7a)<smiles>CCC(=O)CC(C)/C=C(/C#Cc1ccccc1)c1ccccc1</smiles>

Following Method B, $36.2 \mathrm{mg}$ of $7 \mathbf{a}(60 \%$ yield) was obtained as light yellow oil. ${ }^{1} \mathbf{H}$ NMR (400 $\left.\mathbf{M H z}, \mathbf{C D C l}_{3}\right) \delta 7.69-7.62(\mathrm{~m}, 2 \mathrm{H})$, $7.57-7.51(\mathrm{~m}, 2 \mathrm{H}), 7.40-7.33(\mathrm{~m}, 5 \mathrm{H}), 7.30(\mathrm{~d}, J=7.3 \mathrm{~Hz}, 1 \mathrm{H})$, $6.29(\mathrm{~d}, J=9.4 \mathrm{~Hz}, 1 \mathrm{H}), 3.56(\mathrm{ddq}, J=13.7,9.8,6.9 \mathrm{~Hz}, 1 \mathrm{H}), 2.65$ $-2.52(\mathrm{~m}, 2 \mathrm{H}), 2.48(\mathrm{q}, J=7.3 \mathrm{~Hz}, 2 \mathrm{H}), 1.19(\mathrm{~d}, J=6.7 \mathrm{~Hz}, 3 \mathrm{H}), 1.05$ (t, $J=7.3 \mathrm{~Hz}$, 3H); ${ }^{13}$ C NMR (101 MHz, $\left.\mathbf{C D C l}_{3}\right) \delta 210.3,142.2,137.8,131.6,128.4,128.4,127.7$, 126.1, 123.3, 122.9, 95.8, 86.3, 49.3, 36.2, 32.4, 20.3, 7.8; HRMS (ESI) $[\mathrm{M}+\mathrm{H}]^{+}$calcd for $\left[\mathrm{C}_{22} \mathrm{H}_{22} \mathrm{O}+\mathrm{H}\right]^{+}$303.1740, found 303.1743. 
<smiles>CC(=O)CC(C)/C=C(\C#Cc1ccc(C)cc1)c1ccc(C)cc1</smiles>

Following Method B, $38.6 \mathrm{mg}$ of $\mathbf{7 b}$ (61\% yield) was obtained as light yellow oil. ${ }^{1} \mathbf{H}$ NMR $(600 \mathrm{MHz}$, $\left.\mathrm{CDCl}_{3}\right) \delta 7.53(\mathrm{~d}, J=8.0 \mathrm{~Hz}, 2 \mathrm{H}), 7.42(\mathrm{~d}, J=8.0 \mathrm{~Hz}$, 2H), $7.16(\mathrm{~d}, J=7.0 \mathrm{~Hz}, 4 \mathrm{H}), 6.21(\mathrm{~d}, J=9.4 \mathrm{~Hz}, 1 \mathrm{H})$, $3.58-3.50(\mathrm{~m}, 1 \mathrm{H}), 2.63-2.52(\mathrm{~m}, 2 \mathrm{H}), 2.37(\mathrm{~s}, 3 \mathrm{H})$, $2.36(\mathrm{~s}, 3 \mathrm{H}), 2.18(\mathrm{~s}, 3 \mathrm{H}), 1.19(\mathrm{~d}, J=6.7 \mathrm{~Hz}, 3 \mathrm{H})$;

${ }^{13} \mathbf{C}$ NMR (151 MHz, $\left.\mathrm{CDCl}_{3}\right) \delta 208.0,140.7,138.5,137.6,135.1,131.4,129.2,129.1$, 126.0, 123.0, 120.3 95.9, 85.8, 50.8, 32.4, 30.1, 21.5, 21.1, 20.3; HRMS (ESI) $[\mathrm{M}+\mathrm{H}]^{+}$ calcd for $\left[\mathrm{C}_{23} \mathrm{H}_{24} \mathrm{O}+\mathrm{H}\right]^{+} 317.1900$, found 317.1899 .

(Z)-4-Methyl-6,8-di-m-tolyloct-5-en-7-yn-2-one (7c)<smiles>CC(=O)CC(C)/C=C(\C#Cc1cccc(C)c1)c1cccc(C)c1</smiles>

Following Method B, $48.7 \mathrm{mg}$ of 7c (77\% yield) was obtained as light yellow oil. ${ }^{1} \mathbf{H}$ NMR (400 MHz, $\left.\mathrm{CDCl}_{3}\right) \delta 7.48-7.42(\mathrm{~m}, 2 \mathrm{H}), 7.35(\mathrm{~d}, J=7.8 \mathrm{~Hz}, 2 \mathrm{H})$, $7.27(\mathrm{~s}, 1 \mathrm{H}), 7.24(\mathrm{~d}, J=8.1 \mathrm{~Hz}, 1 \mathrm{H}), 7.16(\mathrm{~d}, J=7.4$ $\mathrm{Hz}, 1 \mathrm{H}), 7.11(\mathrm{~d}, J=7.3 \mathrm{~Hz}, 1 \mathrm{H}), 6.26(\mathrm{~d}, J=9.5 \mathrm{~Hz}$, $1 \mathrm{H}), 3.61-3.49(\mathrm{~m}, 1 \mathrm{H}), 2.66-2.54(\mathrm{~m}, 2 \mathrm{H}), 2.39(\mathrm{~s}, 3 \mathrm{H}), 2.37(\mathrm{~s}, 3 \mathrm{H}), 2.20(\mathrm{~s}, 3 \mathrm{H})$, $1.20(\mathrm{~d}, J=6.7 \mathrm{~Hz}, 3 \mathrm{H}) ;{ }^{13} \mathbf{C}$ NMR $\left(101 \mathrm{MHz}, \mathrm{CDCl}_{3}\right) \delta 216.2,207.9,141.7,138.1$, 138.0, 137.8, 132.1, 129.3, 128.7, 128.5, 128.3, 126.7, 123.3, 123.2, 123.1, 95.9, 86.1, 50.7, 32.4, 30.1, 21.5, 21.3, 20.3; HRMS (ESI) $[\mathrm{M}+\mathrm{H}]^{+}$calcd for $\left[\mathrm{C}_{23} \mathrm{H}_{24} \mathrm{O}+\mathrm{H}\right]^{+}$ 317.1900, found 317.1895.

\section{(Z)-6,8-Bis(4-chlorophenyl)-4-methyloct-5-en-7-yn-2-one (7d)}<smiles>CC(=O)CC(C)/C=C(\C#Cc1ccc(Cl)cc1)c1ccc(Cl)cc1</smiles>

Following Method B, $59.1 \mathrm{mg}$ of $7 \mathbf{d}$ (83\% yield) was obtained as light yellow oil. ${ }^{\mathbf{1}} \mathbf{H}$ NMR (400 MHz, $\left.\mathrm{CDCl}_{3}\right) \delta 7.57-7.51(\mathrm{~m}, 2 \mathrm{H}), 7.45(\mathrm{~d}, J=8.7 \mathrm{~Hz}, 2 \mathrm{H})$, $7.37-7.29(\mathrm{~m}, 4 \mathrm{H}), 6.27(\mathrm{~d}, J=9.6 \mathrm{~Hz}, 1 \mathrm{H}), 3.56-$ $3.45(\mathrm{~m}, 1 \mathrm{H}), 2.64-2.54(\mathrm{~m}, 2 \mathrm{H}), 2.17(\mathrm{~s}, 3 \mathrm{H}), 1.18(\mathrm{~d}$, $J=6.8 \mathrm{~Hz}, 3 \mathrm{H}) ;{ }^{13} \mathbf{C ~ N M R}\left(101 \mathrm{MHz}, \mathrm{CDCl}_{3}\right) \delta 207.5$, $142.8,136.1,134.6,133.6,132.8,128.8,128.6,127.3,121.8,121.5,94.9,86.8,50.4$, 
32.3, 30.3, 20.2; HRMS (ESI) $[\mathrm{M}+\mathrm{H}]^{+}$calcd for $\left[\mathrm{C}_{21} \mathrm{H}_{18} \mathrm{Cl}{ }_{2} \mathrm{O}+\mathrm{H}\right]^{+}$357.0807, found 357.0806 .

(Z)-6,8-Bis(4-bromophenyl)-4-methyloct-5-en-7-yn-2-one (7e)

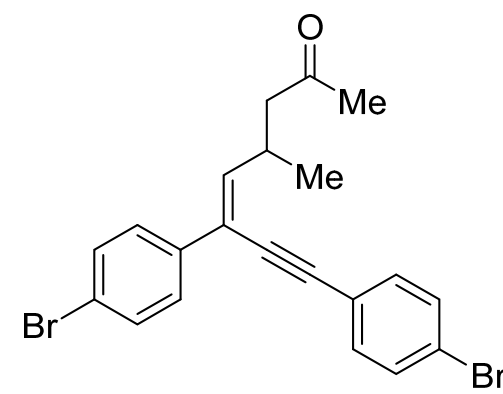

Following Method B, $60.2 \mathrm{mg}$ of 7 e (68\% yield) was obtained as light yellow oil. ${ }^{1} \mathbf{H}$ NMR $(600 \mathrm{MHz}$, $\left.\mathrm{CDCl}_{3}\right) \delta 7.49(\mathrm{~d}, J=8.5 \mathrm{~Hz}, 2 \mathrm{H}), 7.48-7.44(\mathrm{~m}, 4 \mathrm{H})$, $7.37(\mathrm{~d}, J=8.5 \mathrm{~Hz}, 2 \mathrm{H}), 6.28(\mathrm{~d}, J=9.5 \mathrm{~Hz}, 1 \mathrm{H}), 3.53$ $-3.46(\mathrm{~m}, 1 \mathrm{H}), 2.63-2.55(\mathrm{~m}, 2 \mathrm{H}), 2.17(\mathrm{~s}, 3 \mathrm{H}), 1.18$ $(\mathrm{d}, J=6.7 \mathrm{~Hz}, 3 \mathrm{H}) ;{ }^{13} \mathbf{C} \mathbf{N M R}\left(151 \mathrm{MHz}, \mathrm{CDCl}_{3}\right) \delta$ 207.4, 143.0, 136.6, 133.0, 131.7, 131.5, 127.7, 122.8, 122.0, 121.9, 121.8, 95.0, 86.9, 50.4, 32.3, 30.3, 20.1; HRMS (ESI) $[\mathrm{M}+\mathrm{H}]^{+}$calcd for $\left[\mathrm{C}_{21} \mathrm{H}_{18} \mathrm{Br}_{2} \mathrm{O}+\mathrm{H}\right]^{+} 444.9797$, found 444.9795 .

(Z)-6,8-Bis(4-fluorophenyl)-4-methyloct-5-en-7-yn-2-one (7f)<smiles>CC(=O)CC(C)/C=C(\C#Cc1ccc(F)cc1)c1ccc(F)cc1</smiles>

Following Method B, $57.0 \mathrm{mg}$ of $7 \mathbf{f}$ (88\% yield) was obtained as light yellow oil. ${ }^{\mathbf{1}} \mathbf{H}$ NMR $\left(600 \mathrm{MHz}, \mathrm{CDCl}_{3}\right)$ $\delta 7.58(\mathrm{~d}, J=8.5 \mathrm{~Hz}, 2 \mathrm{H}), 7.50(\mathrm{~d}, J=8.4 \mathrm{~Hz}, 2 \mathrm{H}), 7.07$ $-7.02(\mathrm{~m}, 4 \mathrm{H}), 6.20(\mathrm{~d}, J=9.5 \mathrm{~Hz}, 1 \mathrm{H}), 3.55-3.47(\mathrm{~m}$, $1 \mathrm{H}), 2.5-2.63(\mathrm{~m}, 2 \mathrm{H}), 2.18(\mathrm{~s}, 3 \mathrm{H}), 1.19(\mathrm{~d}, J=6.7 \mathrm{~Hz}$, $3 \mathrm{H},) ;{ }^{19}$ F NMR $\left(376 \mathrm{MHz}, \mathrm{CDCl}_{3}\right) \delta-110.54,-114.58$;

${ }^{13}$ C NMR $\left(151 \mathrm{MHz}, \mathrm{CDCl}_{3}\right) \delta 207.7,163.0(\mathrm{~d}, J=249.8 \mathrm{~Hz}), 162.4(\mathrm{~d}, J=248.6 \mathrm{~Hz})$, 142.0, $134.0(\mathrm{~d}, J=3.3 \mathrm{~Hz}), 133.6(\mathrm{~d}, J=8.3 \mathrm{~Hz}), 127.8$ (d, $J=8.1 \mathrm{~Hz}), 122.0,119.3$ (d, $J=3.4 \mathrm{~Hz}), 115.9$ (d, $J=22.2 \mathrm{~Hz}), 115.4$ (d, $J=21.4 \mathrm{~Hz}) ., 95.0,85.9,50.7,32.4$, 30.4, 20.4; HRMS (ESI) $[\mathrm{M}+\mathrm{H}]^{+}$calcd for $\left[\mathrm{C}_{21} \mathrm{H}_{18} \mathrm{~F}_{2} \mathrm{O}+\mathrm{H}\right]^{+} 325.1398$, found 325.1397. (Z)-6,8-Bis(3-chlorophenyl)-4-methyloct-5-en-7-yn-2-one (7g)<smiles>CC(=O)CC(C)/C=C(\C#Cc1cccc(Cl)c1)c1cccc(Cl)c1</smiles>

Following Method B, $35.6 \mathrm{mg}$ of $7 \mathrm{~g}$ (50\% yield) was obtained as light yellow oil. ${ }^{1} \mathbf{H}$ NMR (400 MHz, $\left.\mathrm{CDCl}_{3}\right) \delta 7.58-7.57(\mathrm{~m}, 1 \mathrm{H}), 7.51-7.48(\mathrm{~m}, 2 \mathrm{H}), 7.42$ $-7.38(\mathrm{~m}, 1 \mathrm{H}), 7.34-7.24(\mathrm{~m}, 4 \mathrm{H}), 6.31(\mathrm{~d}, J=9.5 \mathrm{~Hz}$, $1 \mathrm{H}), 3.56-3.45(\mathrm{~m}, 1 \mathrm{H}), 2.65-2.55(\mathrm{~m}, 2 \mathrm{H}), 2.18(\mathrm{~s}$, 
3H), $1.19(\mathrm{~d}, J=6.7 \mathrm{~Hz}, 3 \mathrm{H}) ;{ }^{13} \mathbf{C}$ NMR $\left(101 \mathrm{MHz}, \mathrm{CDCl}_{3}\right) 207.3,143.9,139.4,134.4$, 134.3, 131.4, 129.8, 129.71, 129.67, 128.8, 127.9, 126.2, 124.7, 124.3, 121.6, 94.6, 86.7, 50.4, 32.3, 30.3, 20.1; HRMS (ESI) $[\mathrm{M}+\mathrm{H}]^{+}$calcd for $\left[\mathrm{C}_{21} \mathrm{H}_{18} \mathrm{Cl}_{2} \mathrm{O}+\mathrm{H}\right]^{+} 357.0807$, found 357.0806 .

((Z)-6,8-Bis(3-fluorophenyl)-4-methyloct-5-en-7-yn-2-one (7h)

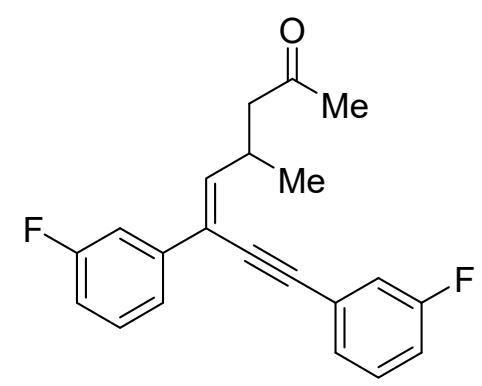

Following Method B, $57.0 \mathrm{mg}$ of $7 \mathbf{h}$ (88\% yield) was obtained as light yellow oil. ${ }^{\mathbf{1}} \mathbf{H} \mathbf{~ N M R}\left(400 \mathrm{MHz}, \mathrm{CDCl}_{3}\right)$ $\delta 7.42-7.39(\mathrm{~m}, 1 \mathrm{H}), 7.34-7.29(\mathrm{~m}, 4 \mathrm{H}), 7.24-7.21$ (m, 1H), $7.09-7.03(\mathrm{~m}, 1 \mathrm{H}), 7.01-6.97(\mathrm{~m}, 1 \mathrm{H}), 6.33$ $(\mathrm{d}, J=9.5 \mathrm{~Hz}, 1 \mathrm{H}), 3.57-3.46(\mathrm{~m}, 1 \mathrm{H}), 2.62-2.55(\mathrm{~m}$,

2H), $2.18(\mathrm{~s}, 3 \mathrm{H}), 1.20(\mathrm{~d}, J=6.7 \mathrm{~Hz}, 3 \mathrm{H}) ;{ }^{19} \mathbf{F} \mathbf{N M R}\left(376 \mathrm{MHz}, \mathrm{CDCl}_{3}\right) \delta-112.69$, 113.18; ${ }^{13}$ C NMR (101 MHz, $\left.\mathrm{CDCl}_{3}\right) \delta 207.5,163.3(\mathrm{~d}, J=245.1 \mathrm{~Hz}), 162.3(\mathrm{~d}, J=$ $246.7 \mathrm{~Hz}), 143.8,140.0$ (d, $J=7.6 \mathrm{~Hz}), 130.2$ (d, $J=8.7 \mathrm{~Hz}), 130.0$ (d, $J=8.4 \mathrm{~Hz})$, $127.6(\mathrm{~d}, J=3.0 \mathrm{~Hz}), 124.9(\mathrm{~d}, J=9.5 \mathrm{~Hz}), 121.9(\mathrm{~d}, J=2.6 \mathrm{~Hz}), 121.8(\mathrm{~d}, J=2.8 \mathrm{~Hz})$, $118.4(\mathrm{~d}, J=22.8 \mathrm{~Hz}), 116.0(\mathrm{~d}, J=21.2 \mathrm{~Hz}), 114.8(\mathrm{~d}, J=21.3 \mathrm{~Hz}), 113.2(\mathrm{~d}, J=22.8$ Hz), 94.8 (d, $J=3.3 \mathrm{~Hz}), 86.7,50.5,32.4,30.4,20.2$; HRMS (ESI) $[\mathrm{M}+\mathrm{H}]^{+}$calcd for $\left[\mathrm{C}_{21} \mathrm{H}_{18} \mathrm{~F}_{2} \mathrm{O}+\mathrm{H}\right]^{+}$325.1398, found 325.1399.

\section{(Z)-6,8-Bis(2-fluorophenyl)-4-methyloct-5-en-7-yn-2-one (7i)}

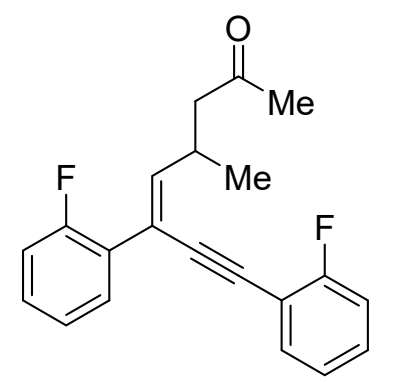

Following Method B, $57.7 \mathrm{mg}$ of $7 \mathbf{i}$ (89\% yield) was obtained as light yellow oil. ${ }^{1} \mathbf{H}$ NMR $\left(400 \mathrm{MHz}, \mathrm{CDCl}_{3}\right) \delta 7.63(\mathrm{td}, J=$ $7.8,1.8 \mathrm{~Hz}, 1 \mathrm{H}), 7.49(\mathrm{td}, J=7.2,1.6 \mathrm{~Hz}, 1 \mathrm{H}), 7.32-7.24$ (m, $2 \mathrm{H}), 7.17-7.14(\mathrm{~m}, 4 \mathrm{H}), 6.37(\mathrm{~d}, J=9.6 \mathrm{~Hz}, 1 \mathrm{H}), 3.62-3.53$ $(\mathrm{m}, 1 \mathrm{H}), 2.68-2.53(\mathrm{~m}, 2 \mathrm{H}), 2.20(\mathrm{~s}, 3 \mathrm{H}), 1.20(\mathrm{~d}, J=6.7 \mathrm{~Hz}$,

$3 \mathrm{H}) ;{ }^{19}$ F NMR $\left(376 \mathrm{MHz}, \mathrm{CDCl}_{3}\right) \delta-109.79,-115.13 ;{ }^{13} \mathbf{C ~ N M R}\left(151 \mathrm{MHz}, \mathrm{CDCl}_{3}\right) \delta$ 207.9, 162.8 (d, $J=253.4 \mathrm{~Hz}), 160.1$ (d, $J=248.8 \mathrm{~Hz}), 147.9$ (d, $J=7.4 \mathrm{~Hz}), 133.4$, 130.5, $130.2(\mathrm{~d}, J=7.5 \mathrm{~Hz}), 129.2$ (d, $J=7.5 \mathrm{~Hz}), 125.8$ (d, $J=11.7 \mathrm{~Hz}), 117.5,116.2$ (d, $J=24.9 \mathrm{~Hz}), 115.6(\mathrm{~d}, J=20.3 \mathrm{~Hz}), 111.9(\mathrm{~d}, J=15.6 \mathrm{~Hz}), 91.5,88.1,50.6,32.7$, 30.1, 20.1; HRMS (ESI) $[\mathrm{M}+\mathrm{H}]^{+}$calcd for $\left[\mathrm{C}_{21} \mathrm{H}_{18} \mathrm{~F}_{2} \mathrm{O}+\mathrm{H}\right]^{+} 325.1398$, found 325.1396. (E)-4-Methyl-6,8-di(thiophen-2-yl)oct-5-en-7-yn-2-one (7j) 
<smiles>CC(=O)CC(C)/C=C(\C#Cc1cccs1)c1cccs1</smiles>

Following Method B, $20.4 \mathrm{mg}$ of $\mathbf{7 j}$ (34\% yield) was obtained as light yellow oil. ${ }^{\mathbf{1}} \mathbf{H}$ NMR $\left(400 \mathrm{MHz}, \mathrm{CDCl}_{3}\right) \delta 7.32(\mathrm{dd}, J=$ $5.2,1.1 \mathrm{~Hz}, 1 \mathrm{H}), 7.29(\mathrm{dd}, J=3.6,1.1 \mathrm{~Hz}, 1 \mathrm{H}), 7.24(\mathrm{dd}, J=3.6$, $1.2 \mathrm{~Hz}, 1 \mathrm{H}), 7.18(\mathrm{dd}, J=5.1,1.2 \mathrm{~Hz}, 1 \mathrm{H}), 7.02(\mathrm{dd}, J=5.1,3.7$ $\mathrm{Hz}, 1 \mathrm{H}), 6.98(\mathrm{dd}, J=5.1,3.7 \mathrm{~Hz}, 1 \mathrm{H}), 6.18(\mathrm{~d}, J=9.5 \mathrm{~Hz}, 1 \mathrm{H})$, $3.67-3.48(\mathrm{~m}, 1 \mathrm{H}), 2.62-2.52(\mathrm{~m}, 2 \mathrm{H}), 2.18(\mathrm{~s}, 3 \mathrm{H}), 1.17(\mathrm{~d}, J=6.7 \mathrm{~Hz}, 3 \mathrm{H}) ;{ }^{13} \mathbf{C}$ NMR $\left(101 \mathrm{MHz}, \mathrm{CDCl}_{3}\right) \delta$ 207.6, 142.2, 140.2, 132.2, 127.7, 127.4, 127.2, 125.0, 124.5, 122.8, 117.2, 88.9, 88.2, 50.5, 32.1, 30.2, 20.2; HRMS (ESI) $[\mathrm{M}+\mathrm{H}]^{+}$calcd for $\left[\mathrm{C}_{17} \mathrm{H}_{16} \mathrm{OSS}_{2}+\mathrm{H}\right]^{+}$301.0715, found 301.0713.

\section{(Z)-3-(2,4-Diphenylbut-1-en-3-yn-1-yl)cyclohexan-1-one (7k)}<smiles>O=C1CCCC(/C=C(/C#Cc2ccccc2)c2ccccc2)C1</smiles>

Following Method B, $36.0 \mathrm{mg}$ of $7 \mathbf{k}$ (60\% yield) was obtained as light yellow oil. ${ }^{1} \mathbf{H}$ NMR (400 MHz, $\left.\mathrm{CDCl}_{3}\right) \delta$ 7.69-7.64 (m, 2H), $7.53-7.49(\mathrm{~m}, 2 \mathrm{H}), 7.40-7.34(\mathrm{~m}, 5 \mathrm{H}), 7.32-7.29(\mathrm{~m}, 1 \mathrm{H}), 6.29(\mathrm{~d}$, $J=9.1 \mathrm{~Hz}, 1 \mathrm{H}), 3.41-3.31(\mathrm{~m}, 1 \mathrm{H}), 2.67-2.61(\mathrm{~m}, 1 \mathrm{H}), 2.49-2.42$ $(\mathrm{m}, 1 \mathrm{H}), 2.39-2.27(\mathrm{~m}, 2 \mathrm{H}), 2.20-2.06(\mathrm{~m}, 2 \mathrm{H}), 1.88-1.77(\mathrm{~m}, 1 \mathrm{H})$, $1.77-1.47(\mathrm{~m}, 2 \mathrm{H}) ;{ }^{13} \mathbf{C}$ NMR $\left(101 \mathrm{MHz}, \mathrm{CDCl}_{3}\right) \delta 210.6,139.6,137.7,131.7,128.64$, $128.61,128.5,128.1,126.2,123.7,123.2,96.2,86.0,47.0,41.4,41.2,31.1,25.4$; HRMS (ESI) $[\mathrm{M}+\mathrm{H}]^{+}$calcd for $\left[\mathrm{C}_{22} \mathrm{H}_{20} \mathrm{O}+\mathrm{H}\right]^{+}$301.1587, found 301.1583 .

(Z)-3-(2,4-Diphenylbut-1-en-3-yn-1-yl)cycloheptan-1-one-(Z)-3-(2,4-diphenylbut1-en-3-yn-1-yl)cycloheptan-1-one (7l)<smiles>O=C1CCCCC(/C=C(/C#Cc2ccccc2)c2ccccc2)C1</smiles>

Following Method B, $28.3 \mathrm{mg}$ of 7l (45\% yield) was obtained as light yellow oil. ${ }^{1} \mathbf{H}$ NMR (400 MHz, $\left.\mathrm{CDCl}_{3}\right) \delta 7.66-7.62(\mathrm{~m}, 2 \mathrm{H}), 7.56-$ $7.53(\mathrm{~m}, 2 \mathrm{H}), 7.40-7.34(\mathrm{~m}, 5 \mathrm{H}), 7.32-7.28(\mathrm{~m}, 1 \mathrm{H}), 6.29(\mathrm{~d}, J=$ $9.2 \mathrm{~Hz}, 1 \mathrm{H}), 3.32-3.22(\mathrm{~m}, 1 \mathrm{H}), 2.77-2.54(\mathrm{~m}, 5 \mathrm{H}), 2.09-1.96(\mathrm{~m}$, 3H), $1.69-1.58(\mathrm{~m}, 4 \mathrm{H}) ;{ }^{13} \mathbf{C} \mathbf{N M R}\left(151 \mathrm{MHz}, \mathrm{CDCl}_{3}\right) \delta 213.2,141.3$, $137.8,131.7,128.6,128.62,128.60,128.0,126.2,123.3,123.0,96.5,86.2,49.3,44.3$, 38.4, 37.0, 29.0, 24.4; HRMS (ESI) $[\mathrm{M}+\mathrm{H}]^{+}$calcd for $\left[\mathrm{C}_{23} \mathrm{H}_{22} \mathrm{O}+\mathrm{H}\right]^{+} 315.1743$, found 315.1743 .

(Z)-3-Methyl-5,7-diphenylhept-4-en-6-ynal (7m) 
<smiles>CC(/C=C(/C#Cc1ccccc1)c1ccccc1)CC=O</smiles>

Following Method B, $30.1 \mathrm{mg}$ of $\mathbf{7 m}$ (55\% yield) was obtained as light yellow oil. ${ }^{1} \mathbf{H}$ NMR $\left(600 \mathrm{MHz}, \mathrm{CDCl}_{3}\right) \delta 9.83(\mathrm{t}, J=2.3 \mathrm{~Hz}, 1 \mathrm{H})$, $7.65(\mathrm{~d}, J=7.3 \mathrm{~Hz}, 2 \mathrm{H}), 7.55-7.51(\mathrm{~m}, 2 \mathrm{H}), 7.39-7.35(\mathrm{~m}, 5 \mathrm{H})$, $7.30(\mathrm{~m}, 1 \mathrm{H}), 6.29(\mathrm{~d}, J=9.4 \mathrm{~Hz}, 1 \mathrm{H}), 3.66-3.56(\mathrm{~m}, 1 \mathrm{H}), 2.62-$ $2.56(\mathrm{~m}, 2 \mathrm{H}), 1.26(\mathrm{~d}, J=6.8 \mathrm{~Hz}, 3 \mathrm{H}) ;{ }^{13} \mathbf{C} \mathbf{N M R}\left(151 \mathrm{MHz}, \mathrm{CDCl}_{3}\right) \delta 201.9,141.1$, 137.6, 131.6, 128.51, 128.47, 128.4, 127.9, 126.1, 123.6, 123.1, 96.2, 86.1, 50.5, 31.1, 20.4; HRMS (ESI) $[\mathrm{M}+\mathrm{H}]^{+}$calcd for $\left[\mathrm{C}_{20} \mathrm{H}_{18} \mathrm{O}+\mathrm{H}\right]^{+}$275.1430, found 275.1427.

\section{(Z)-2-Methyl-5,7-diphenylhept-4-en-6-ynal (7n)}<smiles>CC(C=O)C/C=C(/C#Cc1ccccc1)c1ccccc1</smiles>

Following Method B, $33.4 \mathrm{mg}$ of 7n (61\% yield) was obtained as light yellow oil. ${ }^{1} \mathbf{H}$ NMR (600 MHz, $\left.\mathrm{CDCl}_{3}\right) \delta 9.77(\mathrm{~s}, 1 \mathrm{H}), 7.66(\mathrm{~d}, J=$ $7.4 \mathrm{~Hz}, 2 \mathrm{H}), 7.54-7.52(\mathrm{~m}, 2 \mathrm{H}), 7.39-7.36(\mathrm{~m}, 5 \mathrm{H}), 7.31(\mathrm{t}, J=7.4$ $\mathrm{Hz}, 1 \mathrm{H}), 6.45$ (t, $J=7.6 \mathrm{~Hz}, 1 \mathrm{H}), 2.96(\mathrm{dt}, J=14.0,6.9 \mathrm{~Hz}, 1 \mathrm{H}), 2.76$ $-2.64(\mathrm{~m}, 2 \mathrm{H}), 1.24(\mathrm{~d}, J=7.1 \mathrm{~Hz}, 3 \mathrm{H}) ;{ }^{13} \mathbf{C} \mathbf{~ N M R}\left(151 \mathrm{MHz}, \mathrm{CDCl}_{3}\right)$ $\delta 204.2,137.8,133.9,131.6,128.52,128.49,128.4,127.9,126.1,126.0,123.2,96.2$, 86.3, 46.6, 32.1, 13.4; HRMS (ESI) $[\mathrm{M}+\mathrm{H}]^{+}$calcd for $\left[\mathrm{C}_{20} \mathrm{H}_{18} \mathrm{O}+\mathrm{H}\right]^{+} 275.1430$, found 275.1425 .

\section{Mechanistic Experiments}

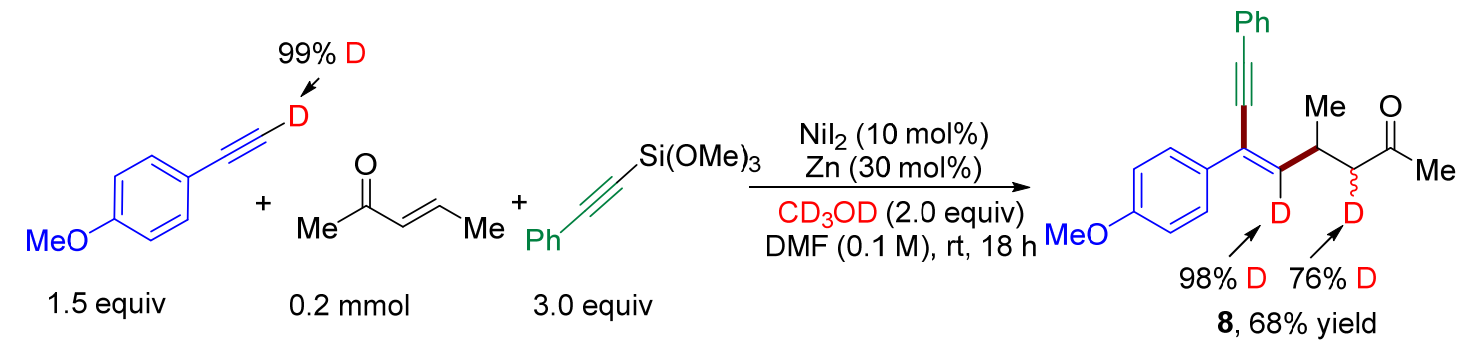

Following Method A: To a Schlenk tube with screw-cap was added $\mathrm{NiI}_{2}(6.3 \mathrm{mg}$, $0.02 \mathrm{mmol}), \mathrm{Zn}(3.9 \mathrm{mg}, 0.06 \mathrm{mmol})$. The tube was evacuated and back-filled with nitrogen (this process was repeated three times). Then DMF $(2.0 \mathrm{~mL})$, alkynyl silicate $(0.60 \mathrm{mmol}, 164 \mu \mathrm{L})$, enone $(0.20 \mathrm{mmol}, 19.5 \mu \mathrm{L})$, alkyne $(0.30 \mathrm{mmol}, 32.9 \mu \mathrm{L})$, and $\mathrm{CD}_{3} \mathrm{OD}(2.0$ equiv, $16.4 \mu \mathrm{L})$ were added consecutively via syringe. The resulting mixture was stirred at room temperature for $18 \mathrm{~h}$. The reaction mixture was quenched with $\mathrm{H}_{2} \mathrm{O}$, extracted with ethyl acetate $(60 \mathrm{~mL})$. The combined organic phase was 
washed with saturated brine $(3 \times 30 \mathrm{~mL})$, dried over anhydrous sodium sulfate, filtered and concentrated under reduced pressure. The crude mixture was purified by column chromatography on silica gel with hexane/ethyl acetate: $20: 1$ as eluent to give the corresponding product $45.5 \mathrm{mg}$ as yellow oil. ${ }^{1} \mathbf{H}$ NMR $\left(400 \mathrm{MHz}, \mathrm{CDCl}_{3}\right) \delta 7.61-$ $7.56(\mathrm{~m}, 2 \mathrm{H}), 7.55-7.50(\mathrm{~m}, 2 \mathrm{H}), 7.39-7.33(\mathrm{~m}, 3 \mathrm{H}), 6.94-6.86(\mathrm{~m}, 2 \mathrm{H}), 3.83(\mathrm{~s}$, $3 \mathrm{H}), 3.58-3.49(\mathrm{~m}, 1 \mathrm{H}), 2.66-2.51(\mathrm{~m}, 1.24 \mathrm{H}), 2.19(\mathrm{~s}, 3 \mathrm{H}), 1.19(\mathrm{~d}, J=6.7 \mathrm{~Hz}, 3 \mathrm{H})$; ${ }^{13}$ C NMR (151 MHz, $\left.\mathrm{CDCl}_{3}\right) \delta 208.0,159.4,139.8(\mathrm{t}, J=22.7 \mathrm{~Hz}), 131.6,130.4,128.5$, 128.4, 127.3, 123.4, 122.3, 113.8, 95.7, 86.5, 55.4, 50.5 (t, $J=18.7 \mathrm{~Hz}$ ), 32.3, 30.2, 20.3; HR-MS (ESI) m/z calcd for $\mathrm{C}_{22} \mathrm{H}_{21} \mathrm{D}_{2} \mathrm{O}_{2}\left[\mathrm{M}+\mathrm{H}^{+}\right]$: 321.1818 , found: 321.1814 .

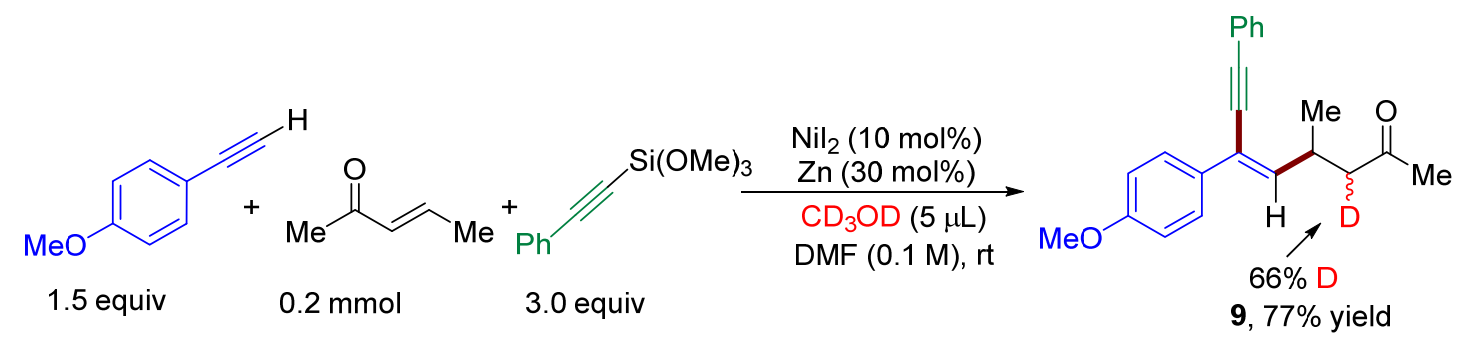

Following Method A: To a Schlenk tube with screw-cap was added $\mathrm{NiI}_{2}$ (6.3 mg, $0.02 \mathrm{mmol}), \mathrm{Zn}(3.9 \mathrm{mg}, 0.06 \mathrm{mmol})$. The tube was evacuated and back-filled with nitrogen (this process was repeated three times). Then DMF $(2.0 \mathrm{~mL})$, alkynyl silicate $(0.60 \mathrm{mmol}, 164 \mu \mathrm{L})$, enone $(0.20 \mathrm{mmol}, 19.5 \mu \mathrm{L})$, alkyne $(0.30 \mathrm{mmol}, 32.9 \mu \mathrm{L})$ and $\mathrm{CD}_{3} \mathrm{OD}(5 \mu \mathrm{L})$ were added consecutively via syringe. The resulting mixture was stirred at room temperature for $18 \mathrm{~h}$. The reaction mixture was quenched with $\mathrm{H}_{2} \mathrm{O}$, extracted with ethyl acetate $(60 \mathrm{~mL})$. The combined organic phase was washed with saturated brine $(3 \times 30 \mathrm{~mL})$, dried over anhydrous sodium sulfate, filtered and concentrated under reduced pressure. The crude mixture was purified by column chromatography on silica gel with hexane/ethyl acetate $=20: 1$ as eluent to give the corresponding product 49.2 mg as yellow oil. ${ }^{1} \mathbf{H}$ NMR $\left(600 \mathrm{MHz}, \mathrm{CDCl}_{3}\right) \delta 7.61-7.56(\mathrm{~m}, 2 \mathrm{H}), 7.55-7.52(\mathrm{~m}$, 2H), $7.39-7.32(\mathrm{~m}, 3 \mathrm{H}), 6.93-6.88(\mathrm{~m}, 2 \mathrm{H}), 6.17(\mathrm{~d}, J=9.4 \mathrm{~Hz}, 1 \mathrm{H}), 3.82(\mathrm{~s}, 1 \mathrm{H})$, $3.56-3.50(\mathrm{~m}, J=13.1,1 \mathrm{H}), 2.65-2.52(\mathrm{~m}, 1.34 \mathrm{H}), 2.19(\mathrm{~s}, 3 \mathrm{H}), 1.19(\mathrm{~d}, J=6.7 \mathrm{~Hz}$, $3 \mathrm{H}) ;{ }^{13} \mathbf{C}$ NMR $\left(101 \mathrm{MHz}, \mathrm{CDCl}_{3}\right) \delta 208.0,159.4,140.2,131.6,130.4,128.5,128.4$, 127.3, 123.4, 122.4, 113.8, 95.7, 86.5, 55.4, 50.5 (t, $J=20.1 \mathrm{~Hz}), 32.4,30.2,20.4$; HRMS (ESI) m/z calcd for $\mathrm{C}_{22} \mathrm{H}_{22} \mathrm{DO}_{2}\left[\mathrm{M}+\mathrm{H}^{+}\right]$: 320.1755 , found: 320.1750 . 


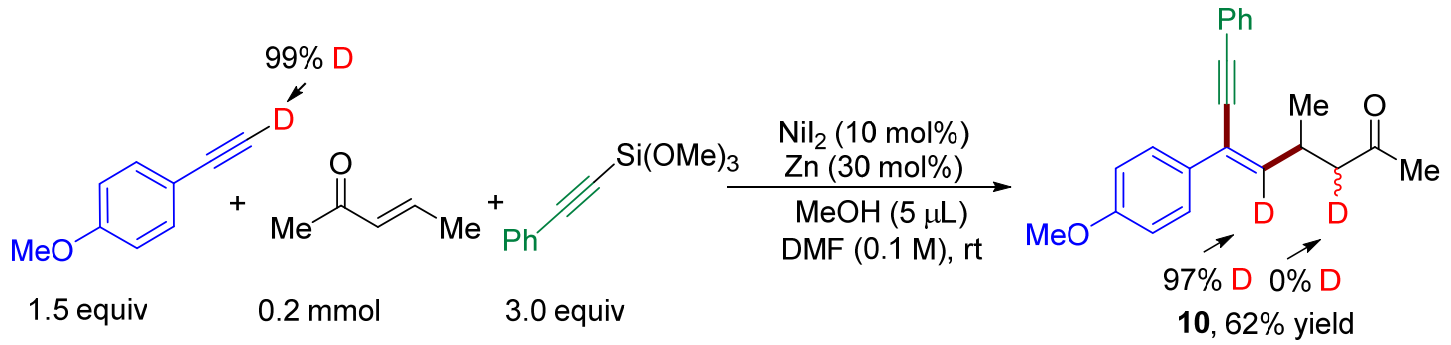

Following Method A: To a Schlenk tube with screw-cap was added NiI 2 (6.3 mg, $0.02 \mathrm{mmol}), \mathrm{Zn}(3.9 \mathrm{mg}, 0.06 \mathrm{mmol})$. The tube was evacuated and back-filled with nitrogen (this process was repeated three times). Then DMF $(2.0 \mathrm{~mL})$, alkynyl silicate $(0.60 \mathrm{mmol}, 164 \mu \mathrm{L})$, enone $(0.20 \mathrm{mmol}, 19.5 \mu \mathrm{L})$, alkyne $(0.30 \mathrm{mmol}, 32.9 \mu \mathrm{L})$ and $\mathrm{MeOH}(5 \mu \mathrm{L})$ were added consecutively via syringe. The resulting mixture was stirred at room temperature for $18 \mathrm{~h}$. The reaction mixture was quenched with $\mathrm{H}_{2} \mathrm{O}$, extracted with ethyl acetate $(60 \mathrm{~mL})$. The combined organic phase was washed with saturated brine $(3 \times 30 \mathrm{~mL})$, dried over anhydrous sodium sulfate, filtered and concentrated under reduced pressure. The crude mixture was purified by column chromatography on silica gel with hexane/ethyl acetate: $20: 1$ as eluent to give the corresponding product 39.6 mg as yellow oil. ${ }^{1} \mathbf{H}$ NMR $\left(400 \mathrm{MHz}, \mathrm{CDCl}_{3}\right) \delta 7.60-7.56(\mathrm{~m}, 2 \mathrm{H}), 7.55-7.51(\mathrm{~m}$, 2H), $7.35(\mathrm{~m}, 3 \mathrm{H}), 6.92-6.87(\mathrm{~m}, 2 \mathrm{H}), 3.83(\mathrm{~s}, 3 \mathrm{H}), 3.57-3.49(\mathrm{~m}, 1 \mathrm{H}), 2.66-2.52$ $(\mathrm{m}, 2 \mathrm{H}), 2.19(\mathrm{~s}, 3 \mathrm{H}), 1.19(\mathrm{~d}, J=6.7 \mathrm{~Hz}, 3 \mathrm{H}) .{ }^{13} \mathbf{C}$ NMR $\left(151 \mathrm{MHz}, \mathrm{CDCl}_{3}\right) \delta 208.0$, 159.5, 139.8 (t, $J=22.7 \mathrm{~Hz}), 131.6,130.4,128.5,128.4,127.3,123.4,122.3,113.8$, 95.7, 86.5, 55.4, 50.9, 32.3, 30.2, 20.4; HR-MS (ESI) $\mathrm{m} / \mathrm{z}$ calcd for $\mathrm{C}_{22} \mathrm{H}_{22} \mathrm{DO}_{2}[\mathrm{M}+$ $\mathrm{H}^{+}$]: 320.1755 , found: 320.1754 .
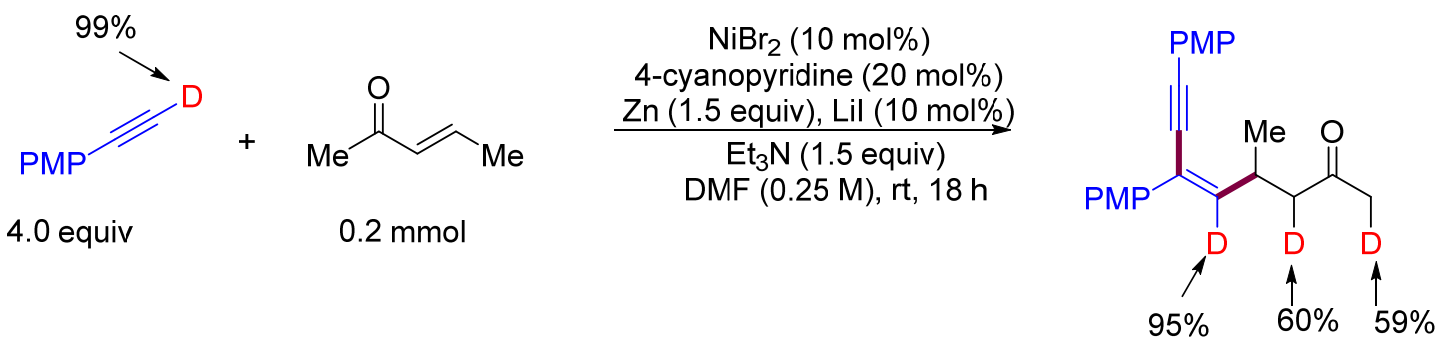

$11,88 \%$ yield

To a Schlenk tube with screw-cap was added NiBr2.DME (6.2 mg, $0.02 \mathrm{mmol}), \mathrm{Zn}$ (19.5 mg, $0.30 \mathrm{mmol}$ ), 4-cyanopyridine (4.2 mg, $0.04 \mathrm{mmol})$, LiI (2.7 mg, $0.02 \mathrm{mmol}$ ) and 1 (106.4 mg, $0.80 \mathrm{mmol})$. The tube was evacuated and back-filled with nitrogen 
(this process was repeated three times). Then DMF $(0.8 \mathrm{~mL}), 2(0.20 \mathrm{mmol})$ and triethylamine $(42.2 \mu \mathrm{L}, 0.30 \mathrm{mmol})$ were added consecutively via syringe. The resulting mixture was stirred at room temperature for $18 \mathrm{~h}$. The reaction mixture was quenched with $\mathrm{H}_{2} \mathrm{O}$, extracted with ethyl acetate $(60 \mathrm{~mL})$. The combined organic phase was washed with saturated brine $(3 \times 30 \mathrm{~mL})$, dried over anhydrous sodium sulfate, filtered and concentrated under reduced pressure. The crude mixture was purified by column chromatography on silica gel with hexane/ethyl acetate mixtures as eluent to give the corresponding product $1161.8 \mathrm{mg}$ as yellow oil. ${ }^{1} \mathbf{H}$ NMR (600 MHz, Chloroform-d) $\delta 7.57(\mathrm{~d}, J=8.3 \mathrm{~Hz}, 2 \mathrm{H}), 7.46(\mathrm{~d}, J=8.3 \mathrm{~Hz}, 2 \mathrm{H}), 6.88(\mathrm{~d}, J=8.2 \mathrm{~Hz}$, $4 \mathrm{H}), 3.83(\mathrm{~s}, 3 \mathrm{H}), 3.82(\mathrm{~s}, 3 \mathrm{H}), 3.51$ (p, J=7.0 Hz, 1H), $2.74-2.44(\mathrm{~m}, 1.4 \mathrm{H}), 2.17(\mathrm{~m}$, 2.41H), $1.18(\mathrm{~d}, J=6.7 \mathrm{~Hz}, 3 \mathrm{H}) ;{ }^{13} \mathbf{C}$ NMR (151 MHz, Chloroform-d) $\delta 208.2,159.6$, $133.1,130.7,127.4,122.6,115.6,114.2,113.9,95.8,85.3,55.49,55.47,50.6(\mathrm{t}, J=$ $19.4 \mathrm{~Hz}$ ), 32.4, 30.0 (t, $J=20.1 \mathrm{~Hz}$ ), 20.5; HR-MS (ESI) m/z calcd for $\mathrm{C}_{23} \mathrm{H}_{21} \mathrm{D}_{3} \mathrm{O}_{3}[\mathrm{M}$ $+\mathrm{H}^{+}$]: 352.1914, found: 352.1975 .

\section{Reference}

1. Fu L., Zhou S., Wan X. L., et al. Enantioselective Trifluoromethylalkynylation of Alkenes via Copper-Catalyzed Radical Relay. J. Am. Chem. Soc. 2018, 140, 10965-10969. 


\section{7. ${ }^{1} \mathrm{H},{ }^{13} \mathrm{C}$, and ${ }^{19} \mathrm{~F}$ Spectra of New Compounds}

\section{(Z)-4-Methyl-6-phenyl-8-(trimethylsilyl)oct-5-en-7-yn-2-one (4a)}

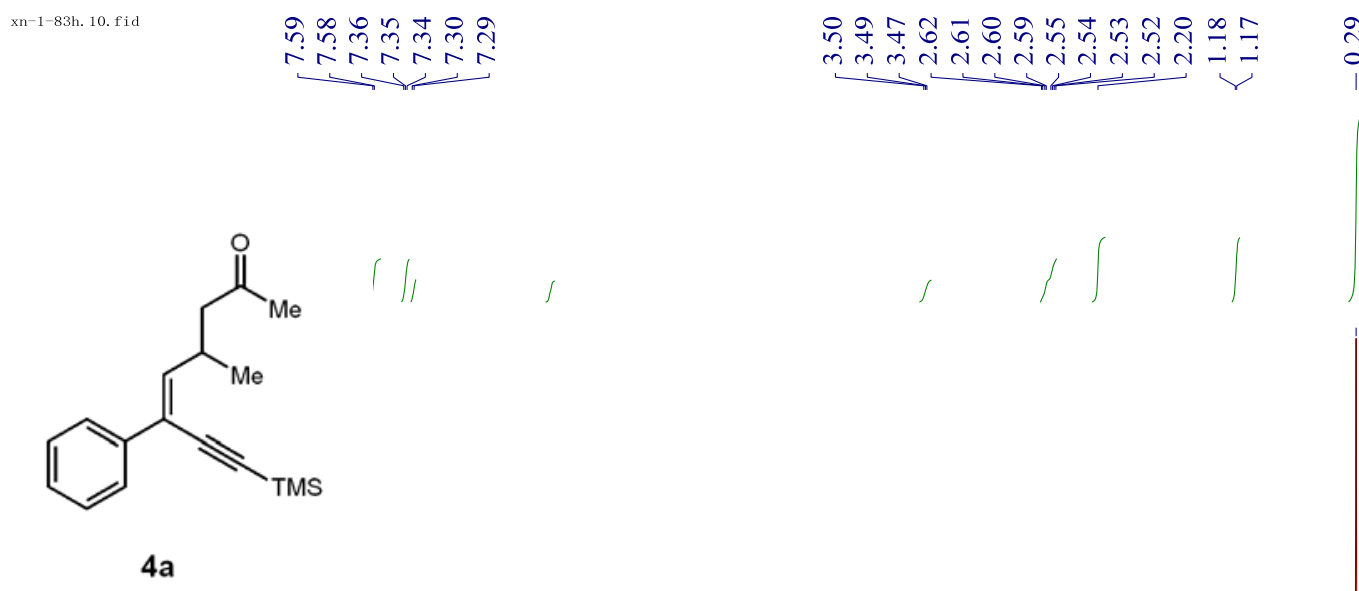

${ }^{1} \mathrm{H} \mathrm{NMR}\left(600 \mathrm{MHz}, \mathrm{CDCl}_{3}\right)$

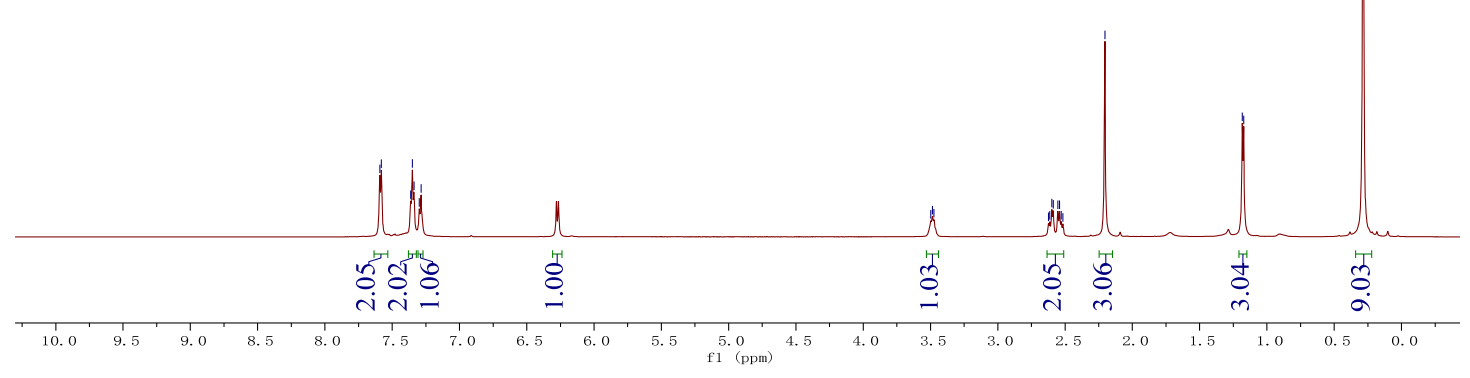

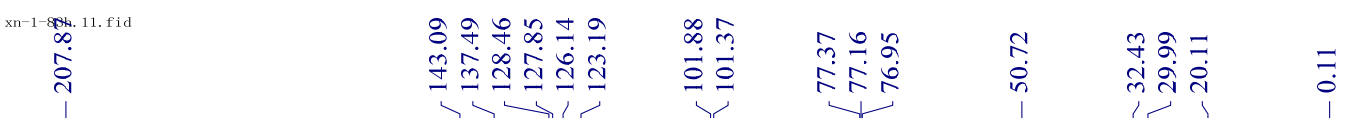<smiles>CC#CC(=CC(C)CC(C)=O)c1ccccc1</smiles>

$4 a$

${ }^{13} \mathrm{C}$ NMR $\left(151 \mathrm{MHz}, \mathrm{CDCl}_{3}\right)$ 


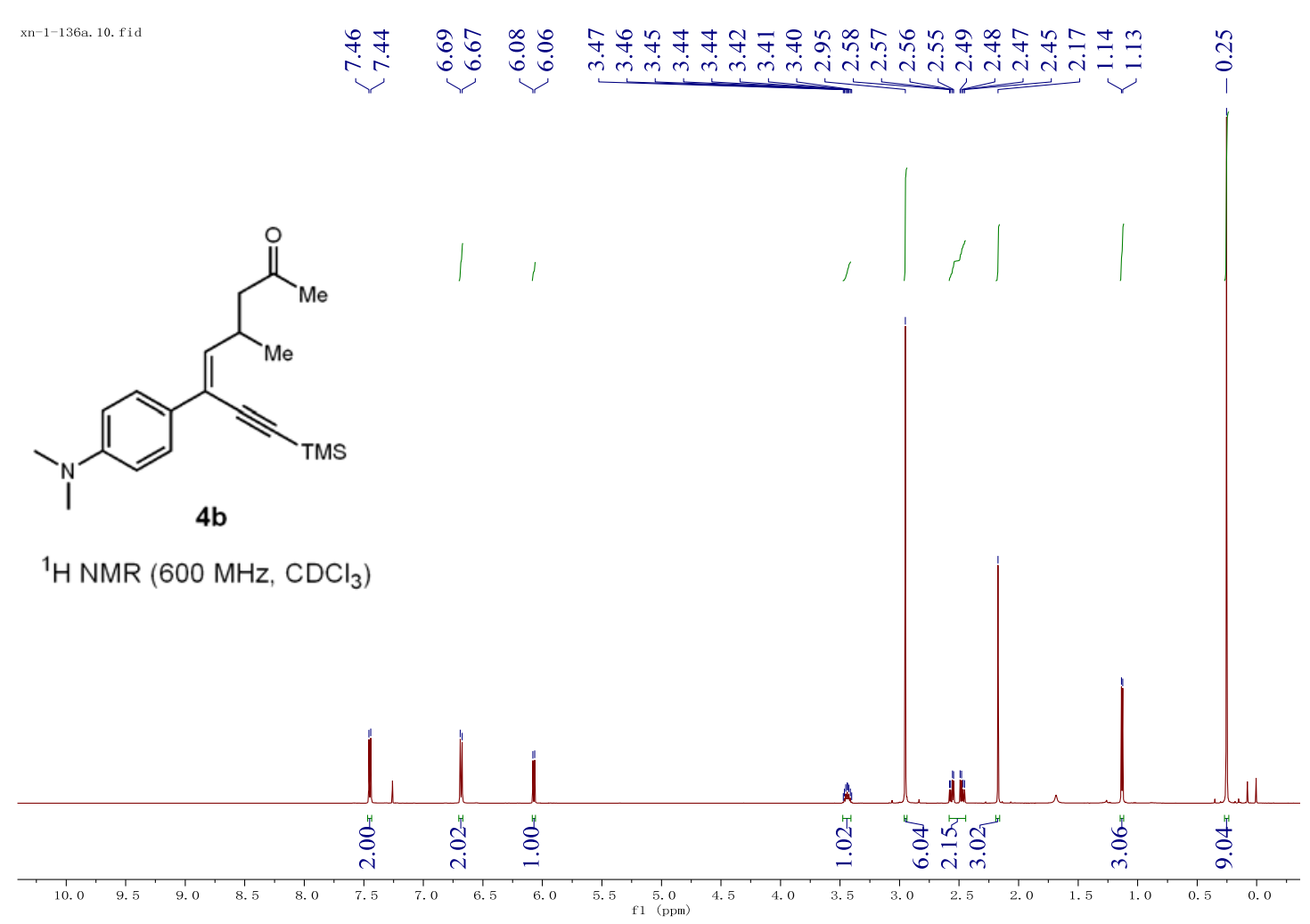

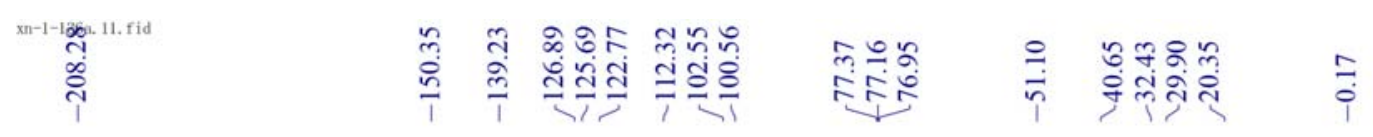<smiles>CC#CC(=CC(C)CC(C)=O)c1ccc(N(C)C)cc1</smiles>

$4 \mathrm{~b}$

${ }^{13} \mathrm{CNMR}\left(151 \mathrm{MHz}, \mathrm{CDCl}_{3}\right)$

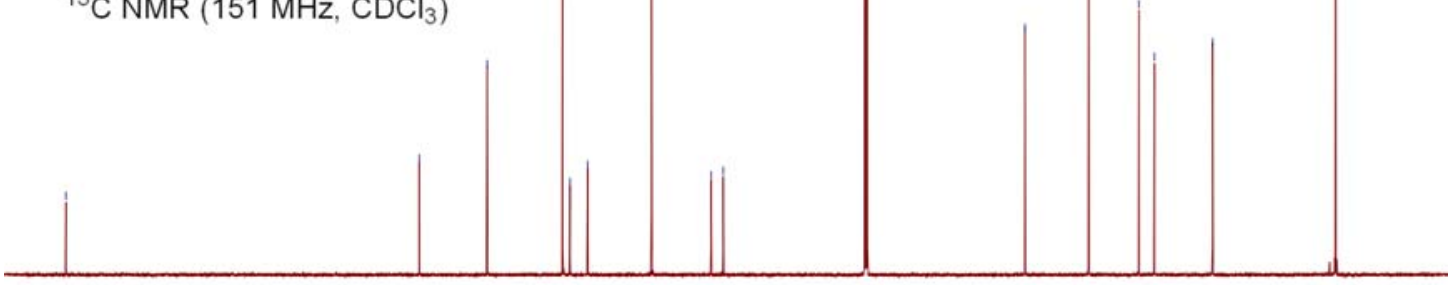

\begin{tabular}{llllllllllllllllllllllllll}
1 & 1 \\
210 & 200 & 190 & 180 & 170 & 160 & 150 & 140 & 130 & 120 & 110 & 100 & 1 & 1 & 1 & 1 & 1 & 1 & 1 & 1 & 1 & 1 & 1 & 1 & 1 & 1 \\
\hline
\end{tabular} 
(Z)-4-Methyl-6-(p-tolyl)-8-(trimethylsilyl)oct-5-en-7-yn-2-one (4c)
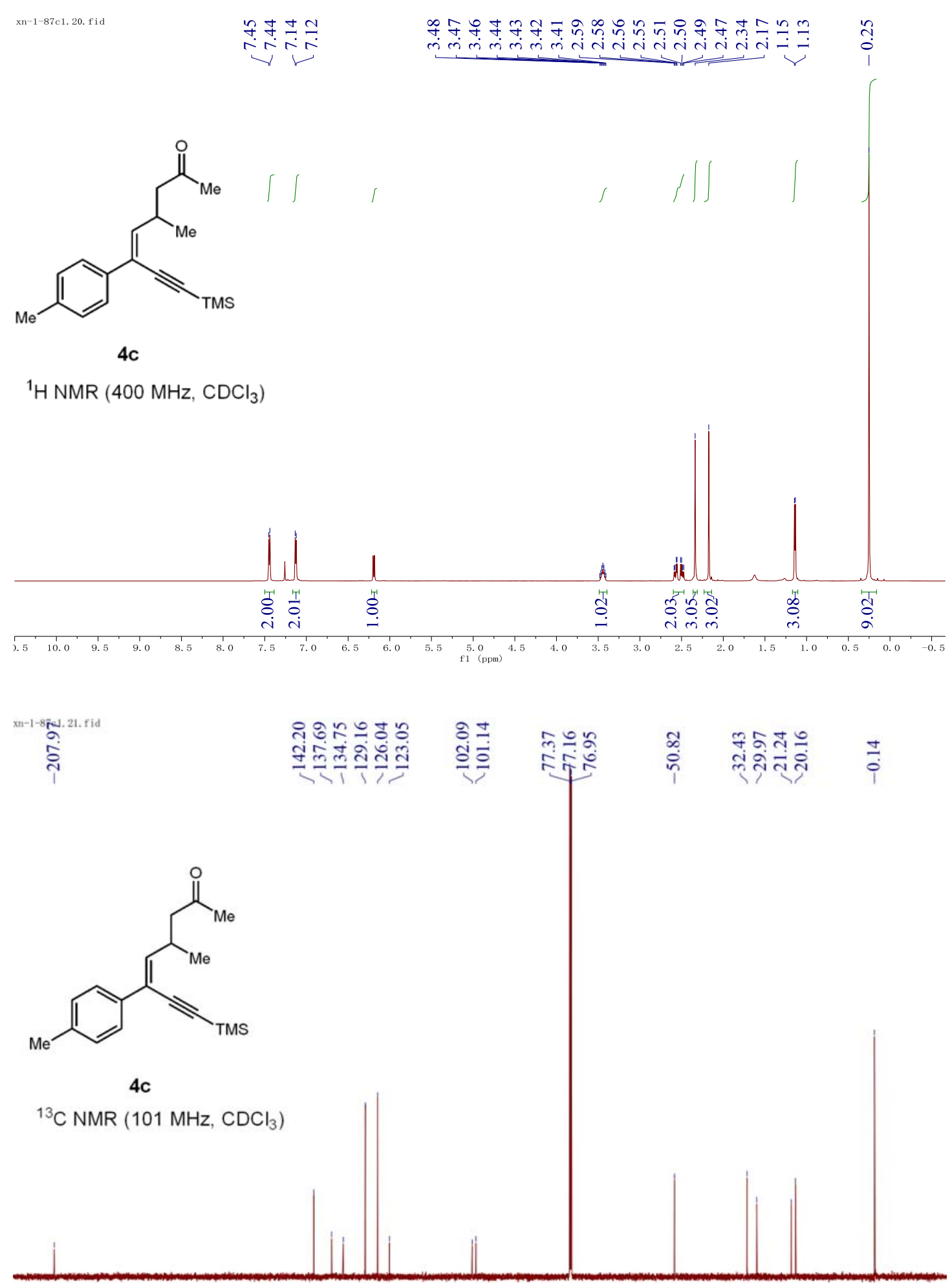
(Z)-6-(4-Methoxyphenyl)-4-methyl-8-(trimethylsilyl)oct-5-en-7-yn-2-one (4d)

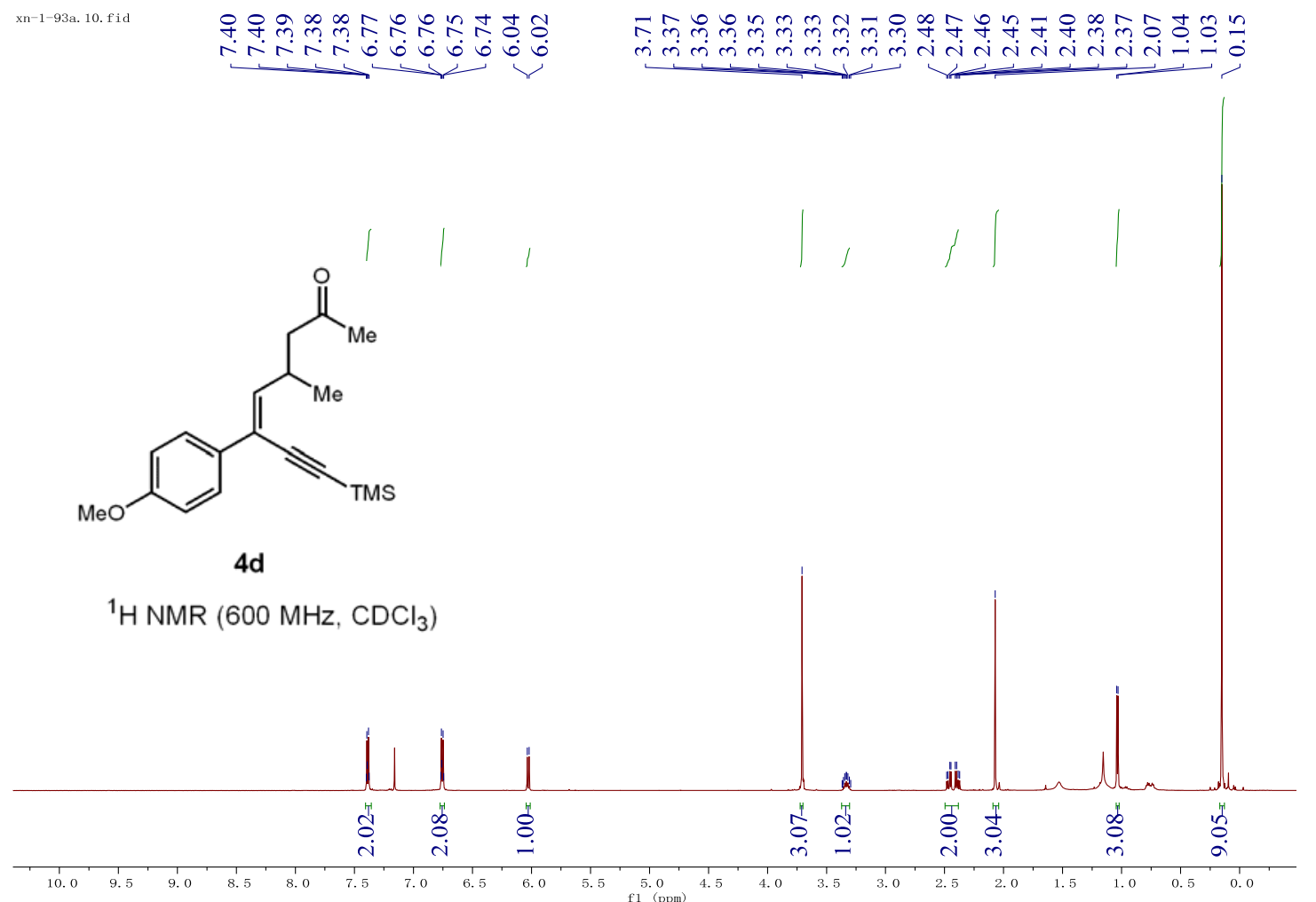

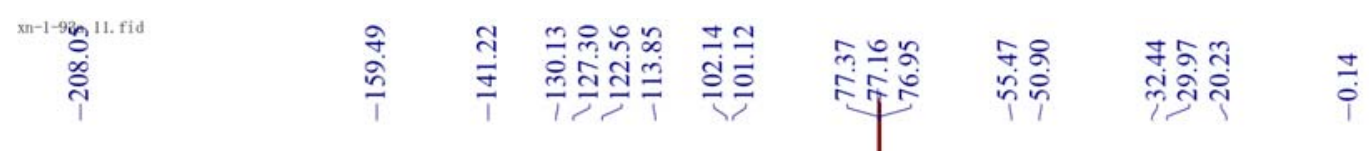<smiles>CC#C/C(=C\C(C)CC(C)=O)c1ccc(OC)cc1</smiles>

4d

${ }^{13} \mathrm{C} \mathrm{NMR}\left(151 \mathrm{MHz}, \mathrm{CDCl}_{3}\right)$

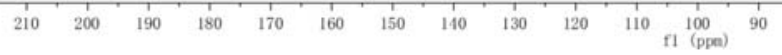




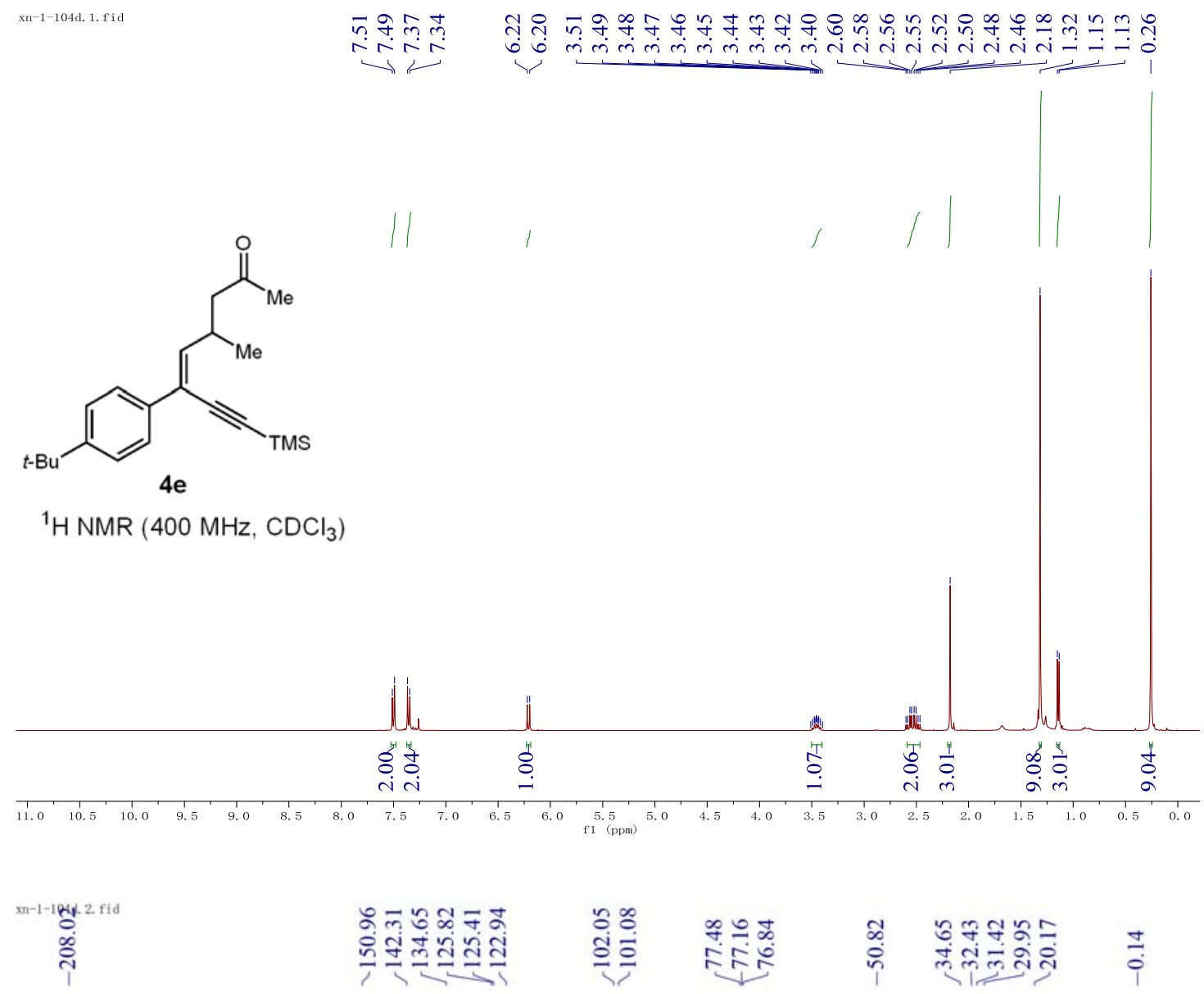<smiles>CC#C/C(=C\C(C)CC(C)=O)c1ccc(C(C)(C)C)cc1</smiles>

${ }^{13} \mathrm{C} \mathrm{NMR}\left(101 \mathrm{MHz}, \mathrm{CDCl}_{3}\right)$

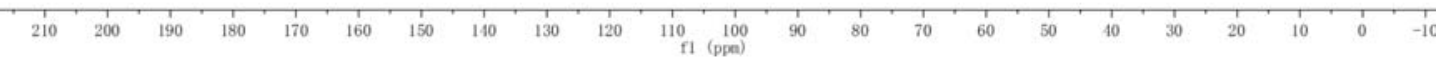




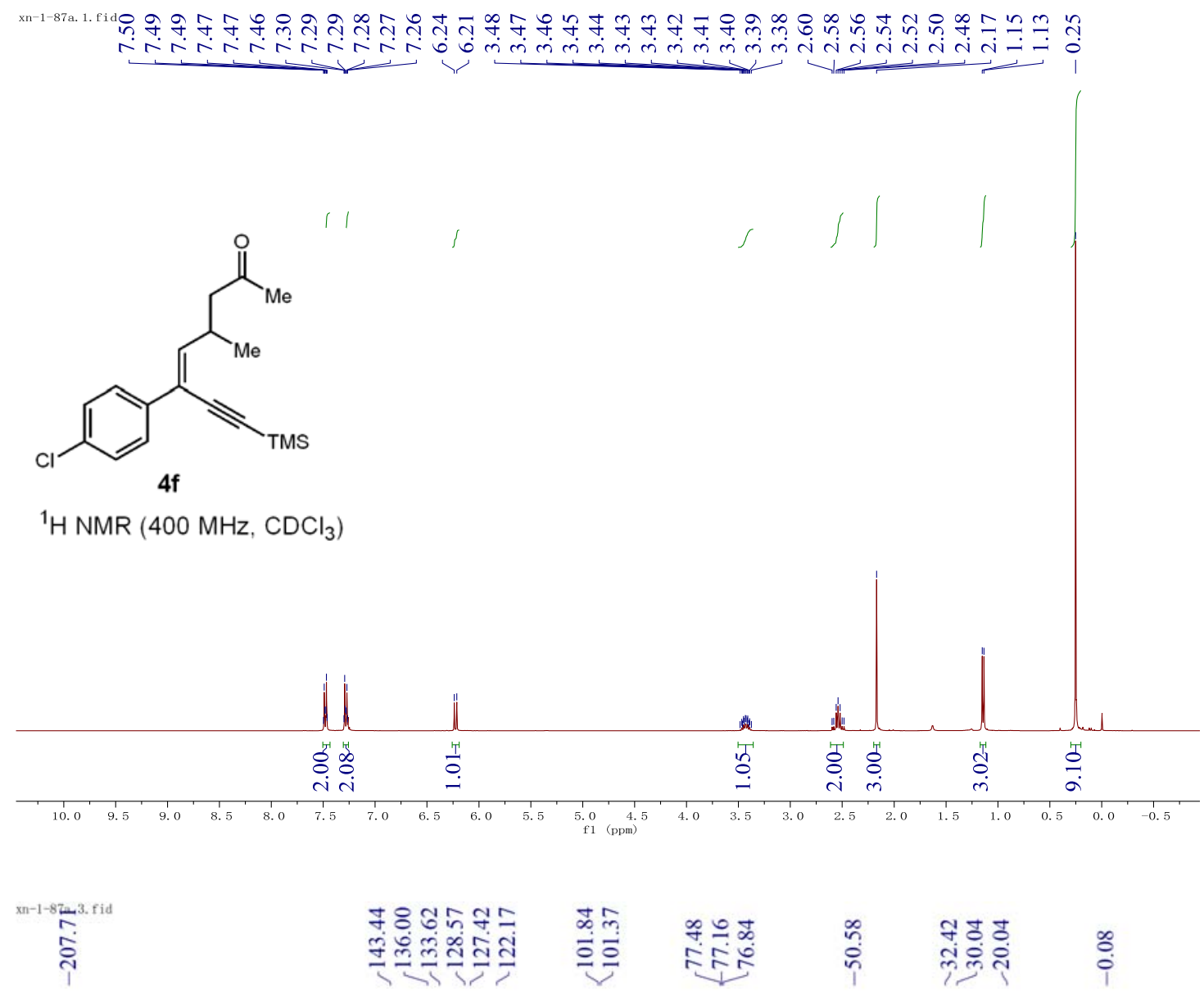<smiles>CC#C/C(=C\C(C)CC(C)=O)c1ccc(Cl)cc1</smiles>

${ }^{13} \mathrm{C}$ NMR $\left(101 \mathrm{MHz}, \mathrm{CDCl}_{3}\right)$

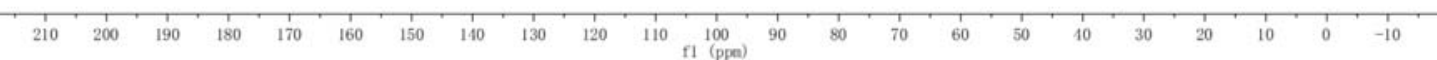


(Z)-6-(4-Bromophenyl)-4-methyl-8-(trimethylsilyl)oct-5-en-7-yn-2-one (4g)
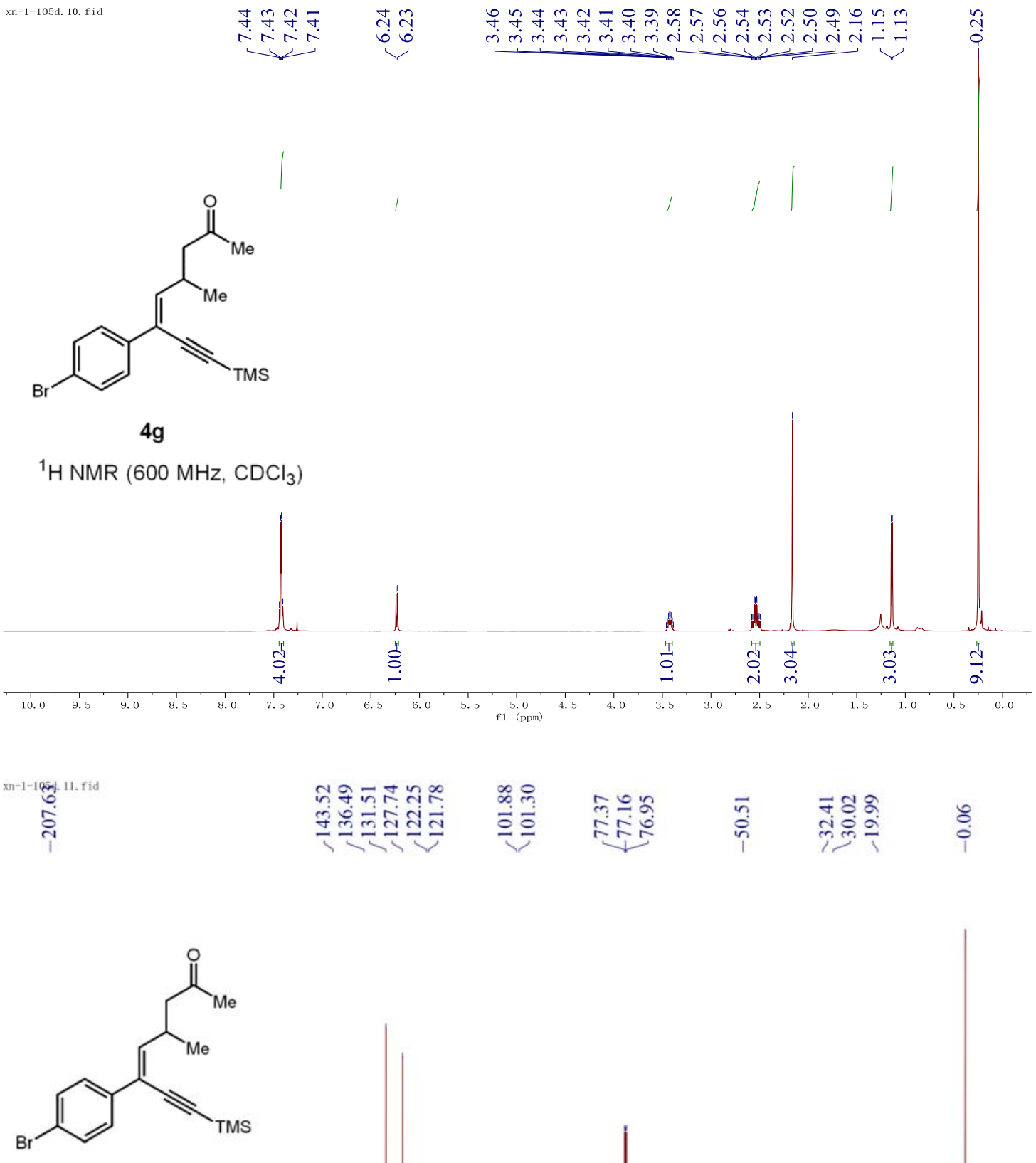

$4 \mathrm{~g}$

${ }^{13} \mathrm{C} \mathrm{NMR}\left(151 \mathrm{MHz}, \mathrm{CDCl}_{3}\right)$

$\begin{array}{llllllllllll}210 & 200 & 190 & 180 & 170 & 160 & 150 & 140 & 130 & 120 & 110 & 100 \\ \mathrm{f} 1 & 1 & 1 & 1 \\ (\mathrm{ppa}) & 1\end{array}$ 
Methyl (Z)-4-(5-methyl-7-oxo-1-(trimethylsilyl)oct-3-en-1-yn-3-yl)benzoate (4h)

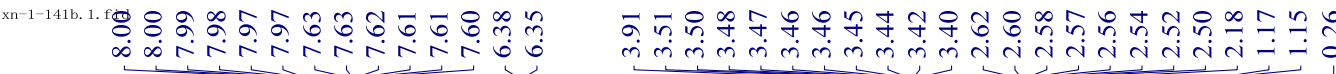<smiles>CC#C/C(=C\C(C)CC(C)=O)c1ccc(C(C)=O)cc1</smiles>

4h

${ }^{1} \mathrm{H}$ NMR $\left(400 \mathrm{MHz}, \mathrm{CDCl}_{3}\right)$

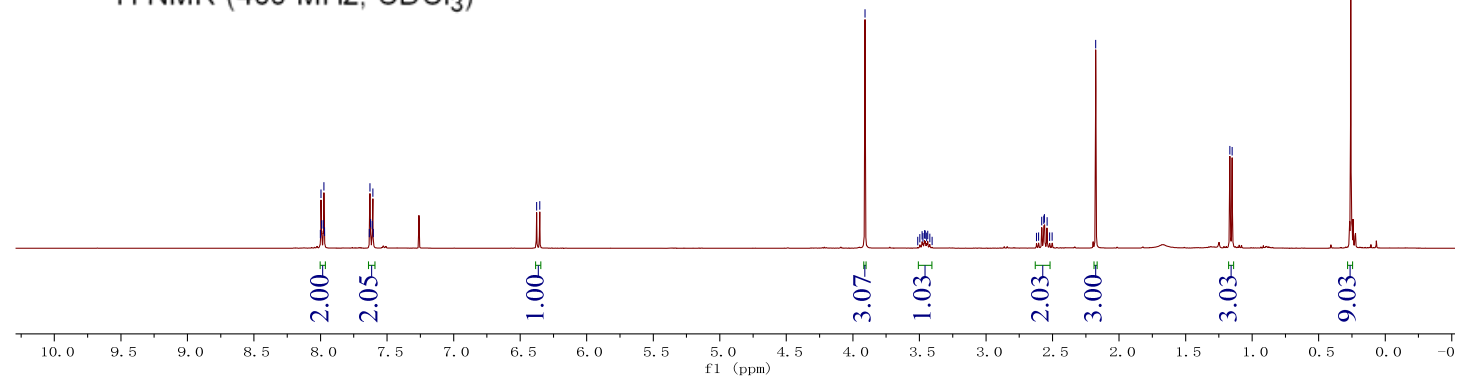

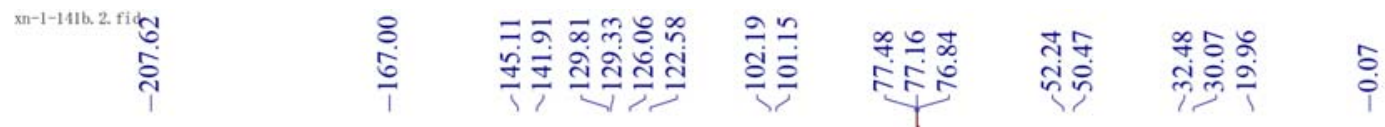<smiles>CC#C/C(=C\C(C)CC(C)=O)c1ccc(C(C)=O)cc1</smiles>

$4 \mathrm{~h}$

${ }^{13} \mathrm{C}$ NMR $\left(101 \mathrm{MHz}, \mathrm{CDCl}_{3}\right)$

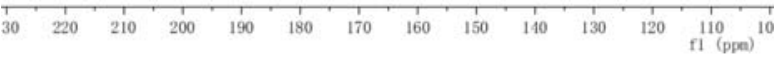




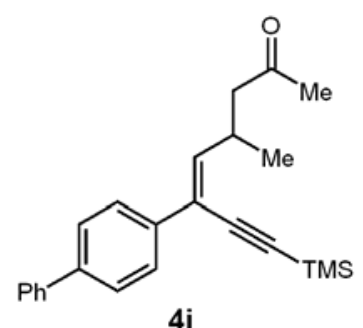

${ }^{1} \mathrm{H} \mathrm{NMR}\left(400 \mathrm{MHz}, \mathrm{CDCl}_{3}\right)$

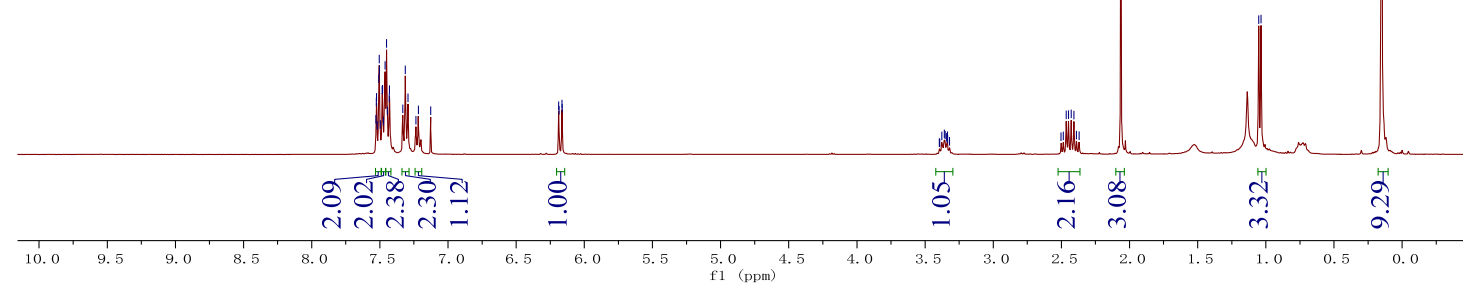

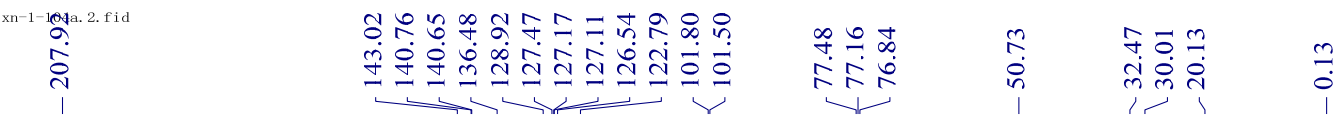<smiles>CC#C/C(=C\C(C)CC(C)=O)c1ccc(-c2ccccc2)cc1</smiles>

${ }^{13} \mathrm{C} \mathrm{NMR}\left(101 \mathrm{MHz}, \mathrm{CDCl}_{3}\right)$

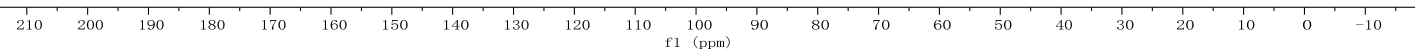


(Z)-6-(4-Fluorophenyl)-4-methyl-8-(trimethylsilyl)oct-5-en-7-yn-2-one (4j)

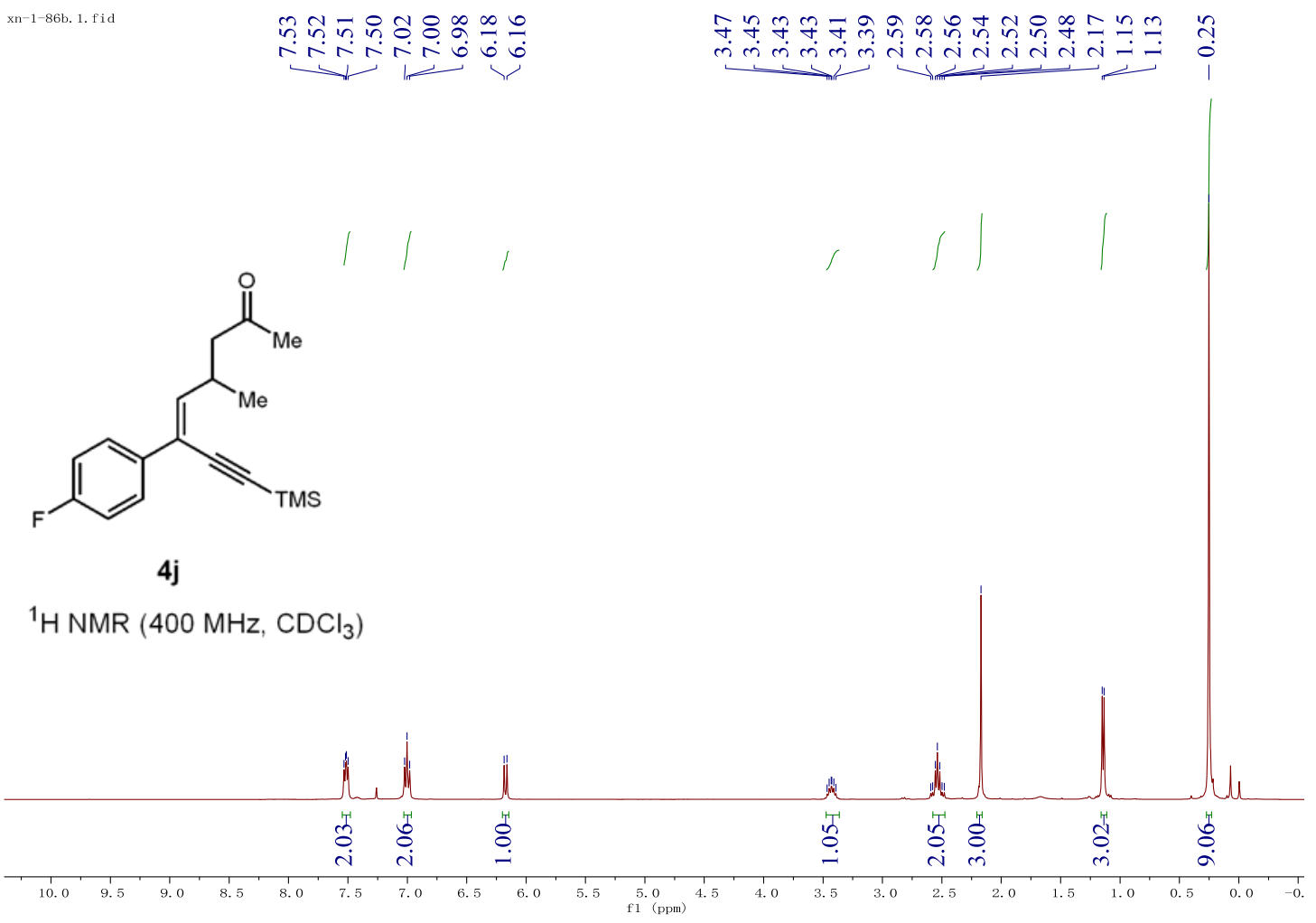

xn-1-86b. 2. fid

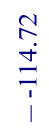<smiles>CC(=O)CC(C)C=C(C#CN)c1ccc(F)cc1</smiles>

4j

${ }^{19} \mathrm{~F} \mathrm{NMR}\left(376 \mathrm{MHz}, \mathrm{CDCl}_{3}\right)$

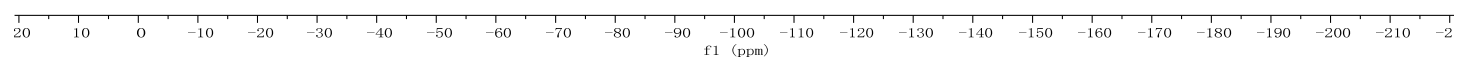




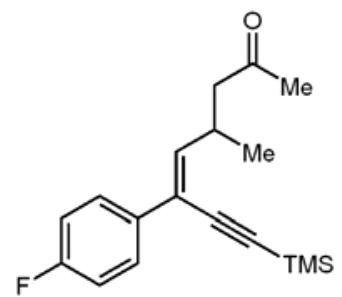

$4 \mathrm{j}$

${ }^{13} \mathrm{C}$ NMR $\left(101 \mathrm{MHz}, \mathrm{CDCl}_{3}\right)$
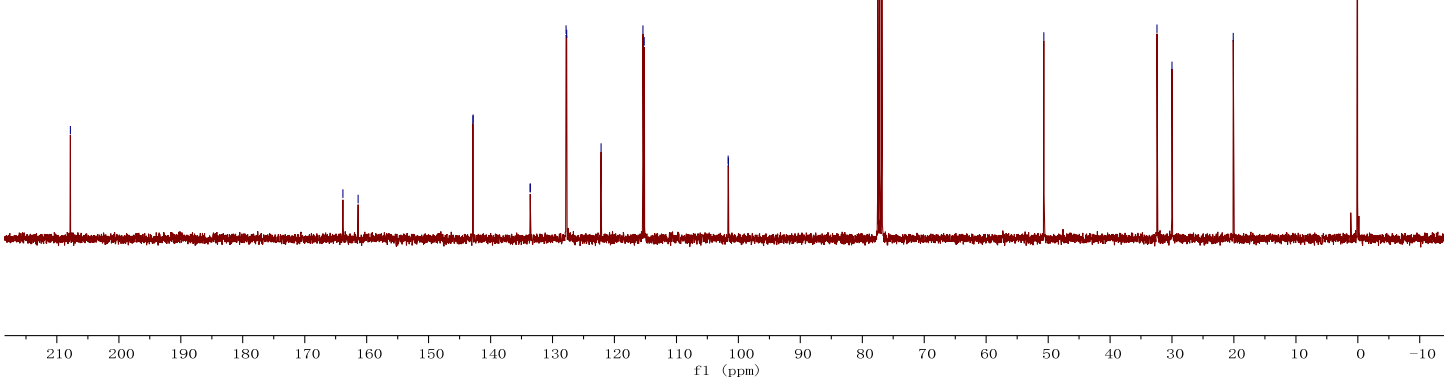

(Z)-6-(2-Methoxyphenyl)-4-methyl-8-(trimethylsilyl)oct-5-en-7-yn-2-one (4k)

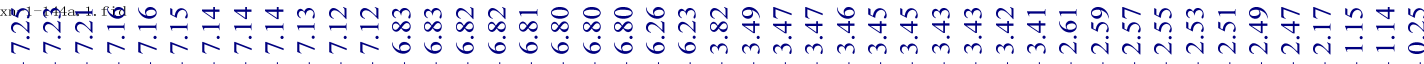<smiles>CC#C/C(=C\C(C)CC(C)=O)c1cccc(OC)c1</smiles>

$4 \mathrm{k}$

${ }^{1} \mathrm{H}$ NMR $\left(400 \mathrm{MHz}, \mathrm{CDCl}_{3}\right)$

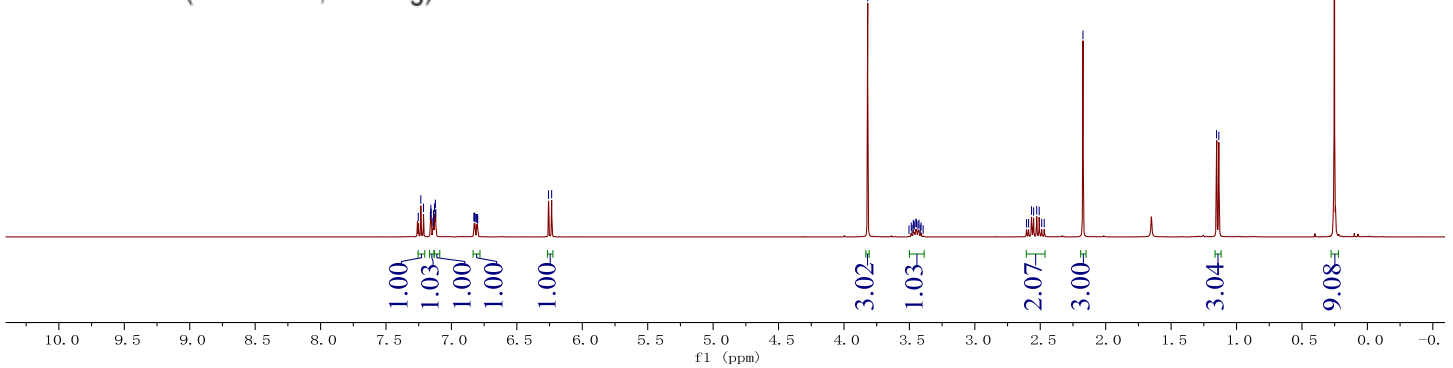




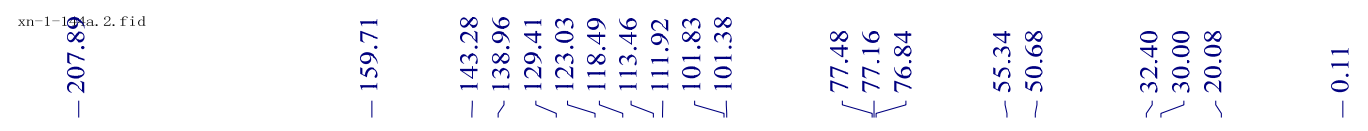<smiles>CC#C/C(=C\C(C)CC(C)=O)c1cccc(OC)c1</smiles>

4k

${ }^{13} \mathrm{C}$ NMR $\left(101 \mathrm{MHz}, \mathrm{CDCl}_{3}\right)$

(Z)-4-Methyl-6-(m-tolyl)-8-(trimethylsilyl)oct-5-en-7-yn-2-one (4l)

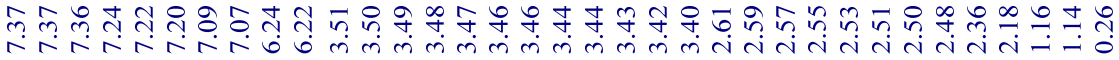<smiles>CC#C/C(=C\C(C)CC(C)=O)c1cccc(C)c1</smiles>

${ }^{1} \mathrm{H}$ NMR $\left(400 \mathrm{MHz}, \mathrm{CDCl}_{3}\right)$

Lur

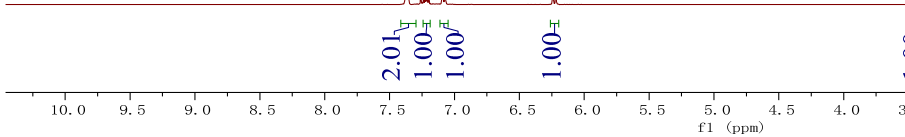

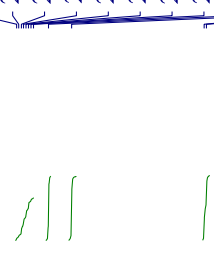

情

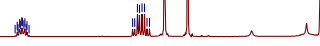

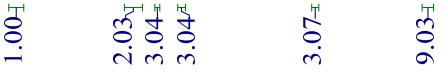


<smiles>CC#C/C(=C\C(C)CC(C)=O)c1cccc([N+](=O)[O-])c1</smiles>

$\left.{ }^{13} \mathrm{C} \mathrm{NMR} \mathrm{(101} \mathrm{MHz,} \mathrm{CDCl}_{3}\right)$

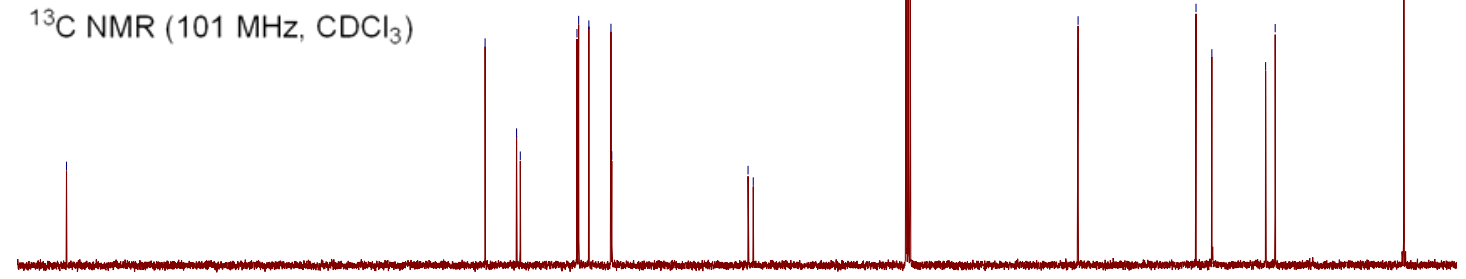

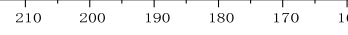<smiles>CC#C/C(=C\C(C)CC(C)=O)c1cccc(F)c1</smiles>

$4 m$

${ }^{1} \mathrm{H} \mathrm{NMR}\left(400 \mathrm{MHz}, \mathrm{CDCl}_{3}\right)$
Wh

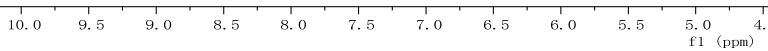




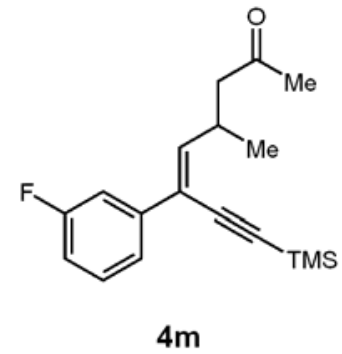

${ }^{19} \mathrm{~F} \mathrm{NMR}\left(376 \mathrm{MHz}, \mathrm{CDCl}_{3}\right)$

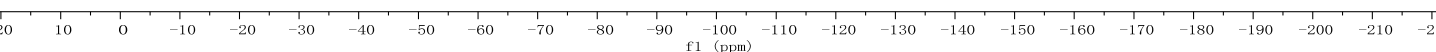

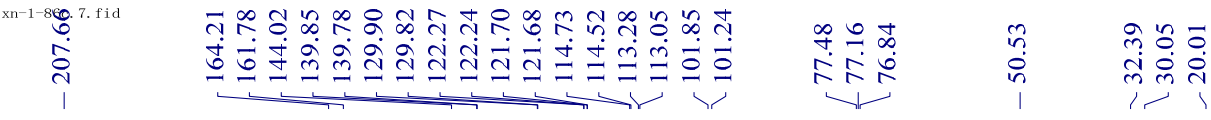<smiles>CC#C/C(=C/C(C)CC(C)=O)c1cccc(F)c1</smiles>

$4 m$

${ }^{13} \mathrm{C}$ NMR $\left(101 \mathrm{MHz}, \mathrm{CDCl}_{3}\right)$

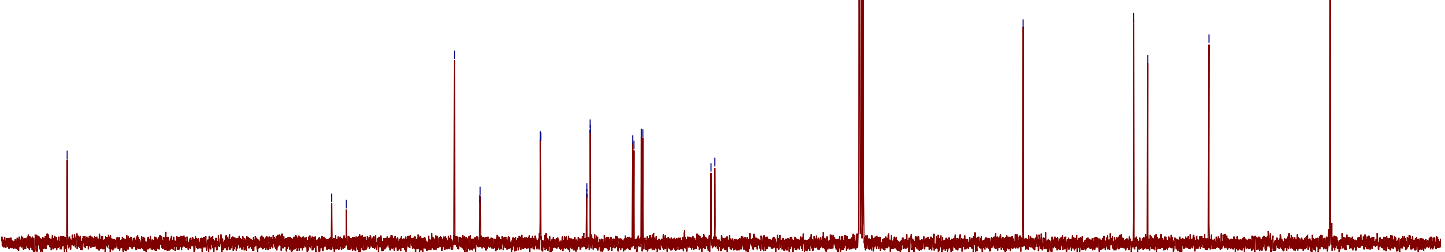




\section{(Z)-6-(3-Chlorophenyl)-4-methyl-8-(trimethylsilyl)oct-5-en-7-yn-2-one (4n)}

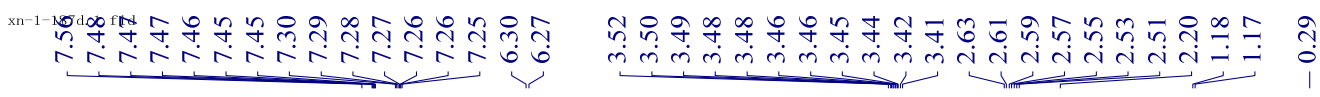<smiles>CC#C/C(=C\C(C)CC(C)=O)c1cccc(Cl)c1</smiles>

$4 n$

${ }^{1} \mathrm{H} \mathrm{NMR}\left(400 \mathrm{MHz}, \mathrm{CDCl}_{3}\right)$

ì

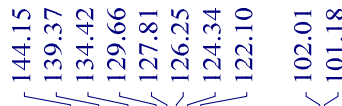

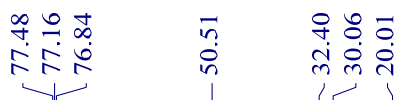

$\stackrel{8}{:}$<smiles>CC#C/C(=C\C(C)CC(C)=O)c1cccc(Cl)c1</smiles>

$4 \mathrm{n}$

${ }^{13} \mathrm{C} \mathrm{NMR}\left(101 \mathrm{MHz}, \mathrm{CDCl}_{3}\right)$

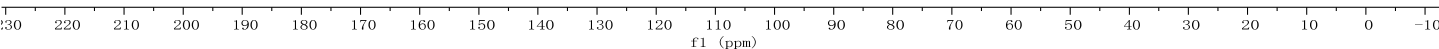


<smiles>COc1ccccc1/C(C#CN)=C/C(C)CC(C)=O</smiles>

40

${ }^{1} \mathrm{H}$ NMR $\left(400 \mathrm{MHz}, \mathrm{CDCl}_{3}\right)$

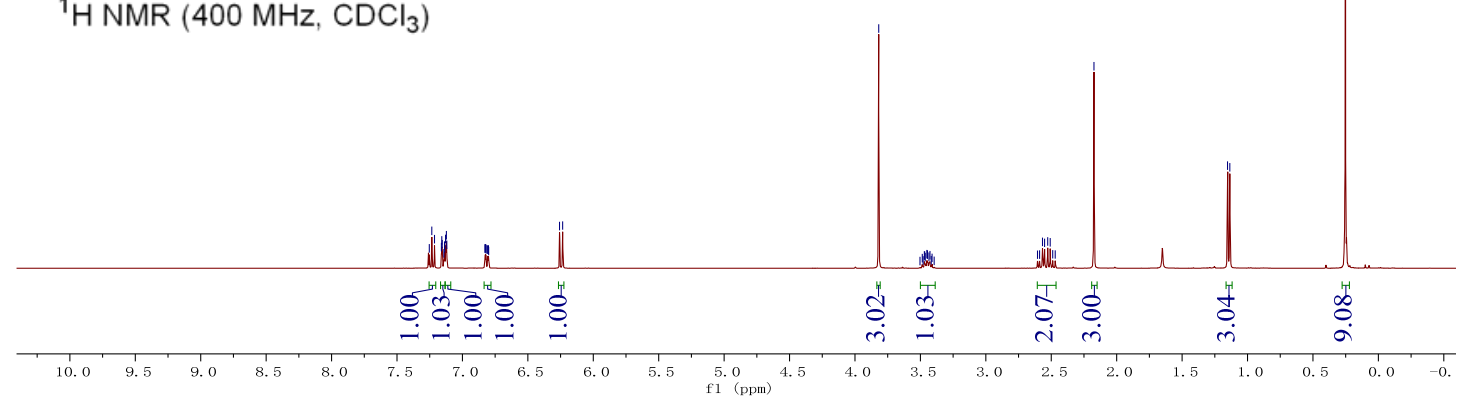

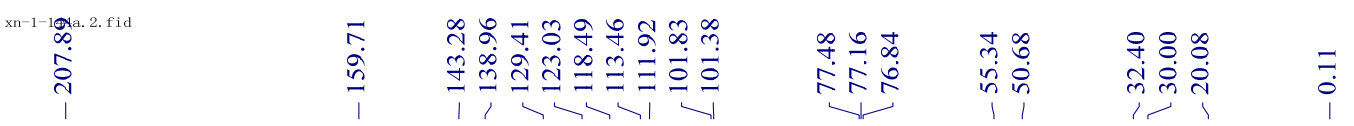<smiles>CC#C/C(=C\C(C)CC(C)=O)c1ccccc1OC</smiles>

40

${ }^{13} \mathrm{C}$ NMR $\left(101 \mathrm{MHz}, \mathrm{CDCl}_{3}\right)$

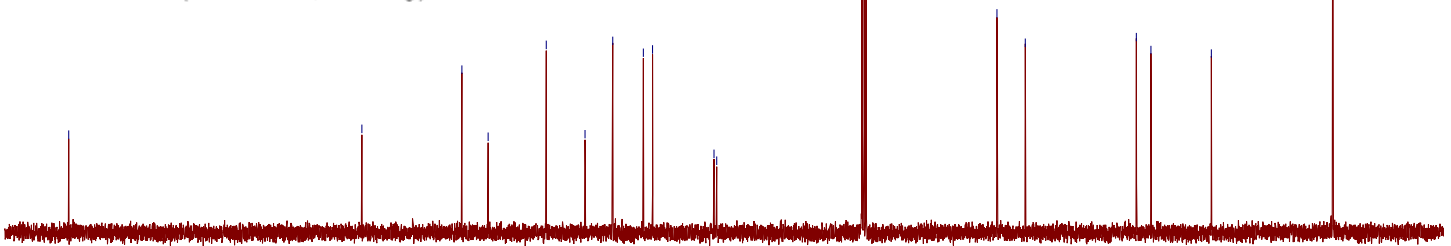




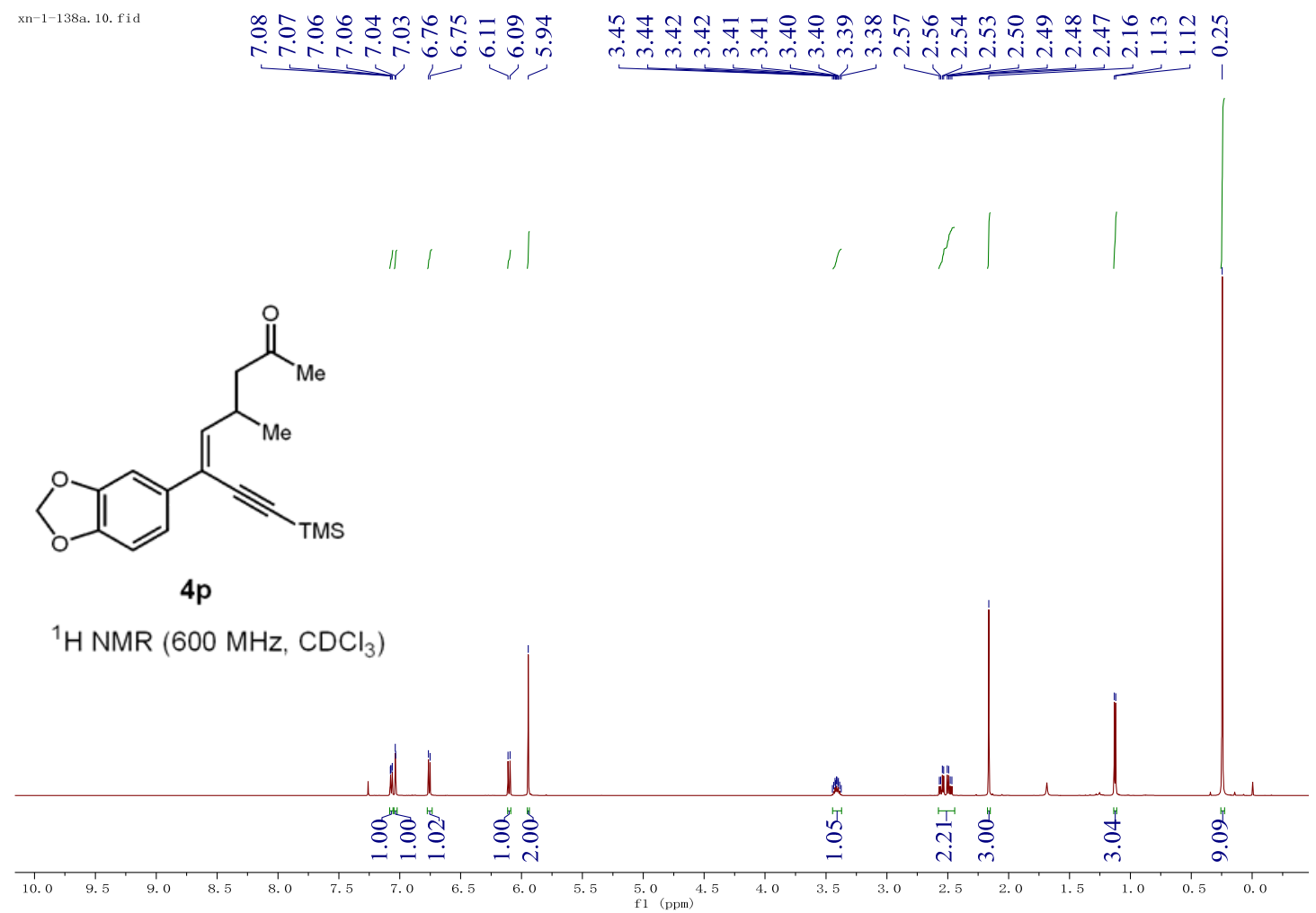

\begin{tabular}{|c|}
\hline 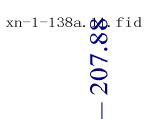 \\
\hline
\end{tabular}

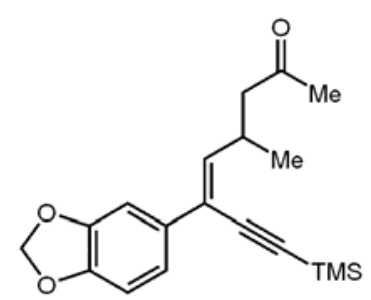

$4 p$

${ }^{13} \mathrm{C}$ NMR $\left(151 \mathrm{MHz}, \mathrm{CDCl}_{3}\right)$

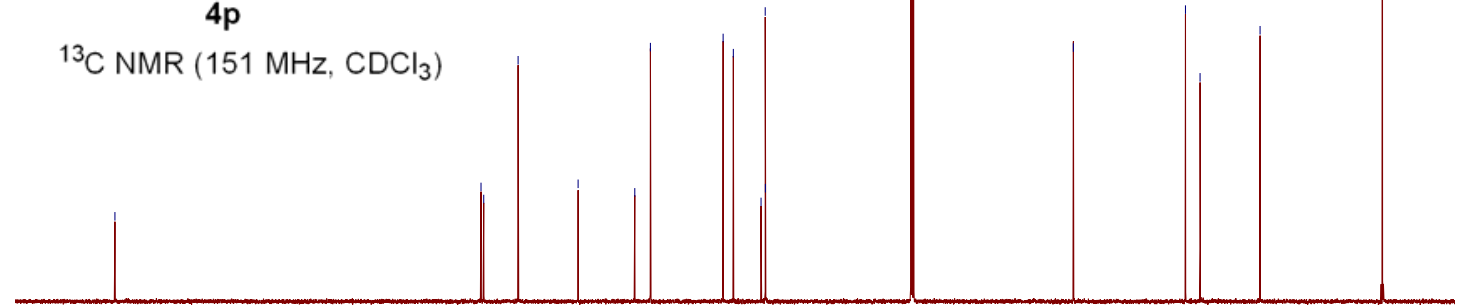




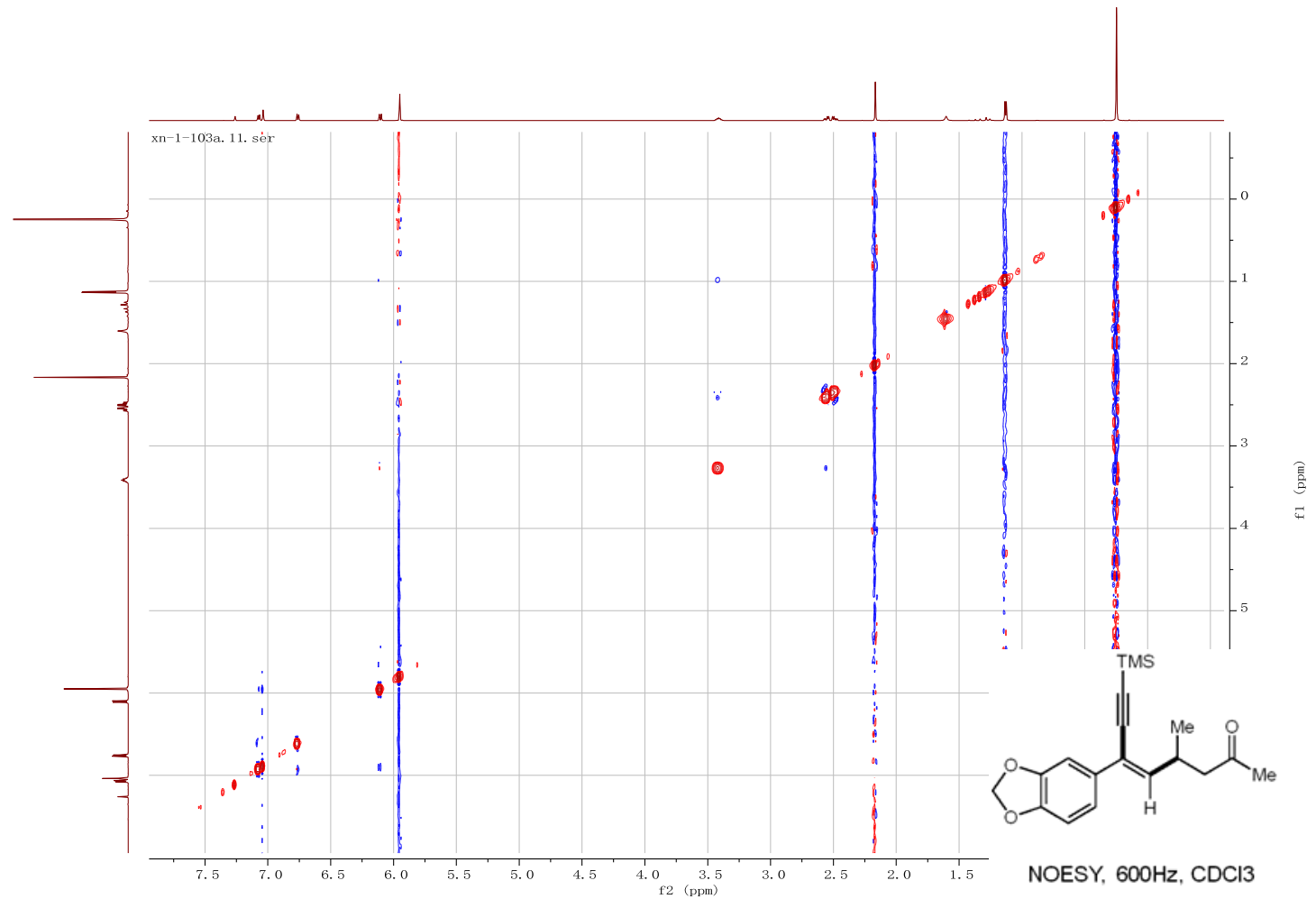

(Z)-4-Methyl-6-(naphthalen-2-yl)-8-(trimethylsilyl)oct-5-en-7-yn-2-one (4q)

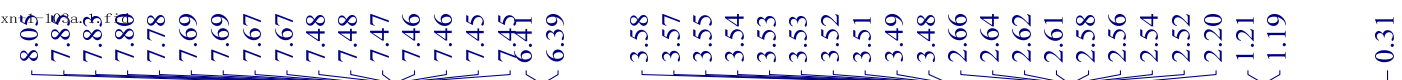

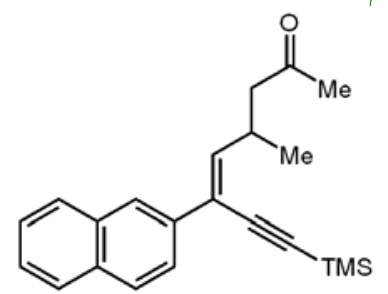

$4 q$

${ }^{1} \mathrm{H}$ NMR $\left(400 \mathrm{MHz}, \mathrm{CDCl}_{3}\right)$

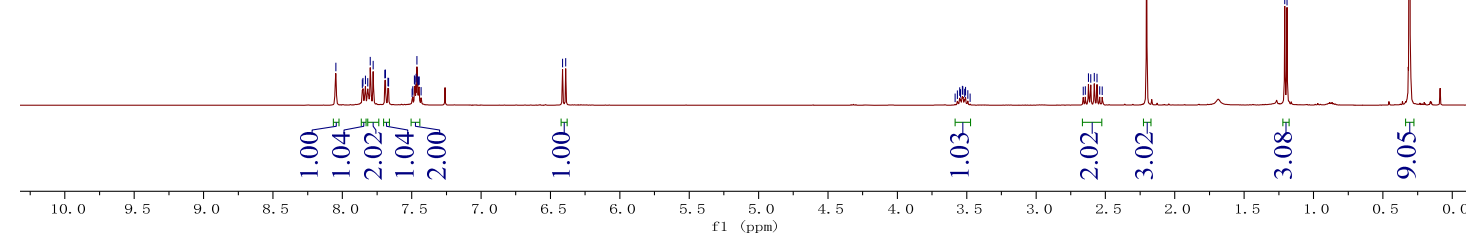



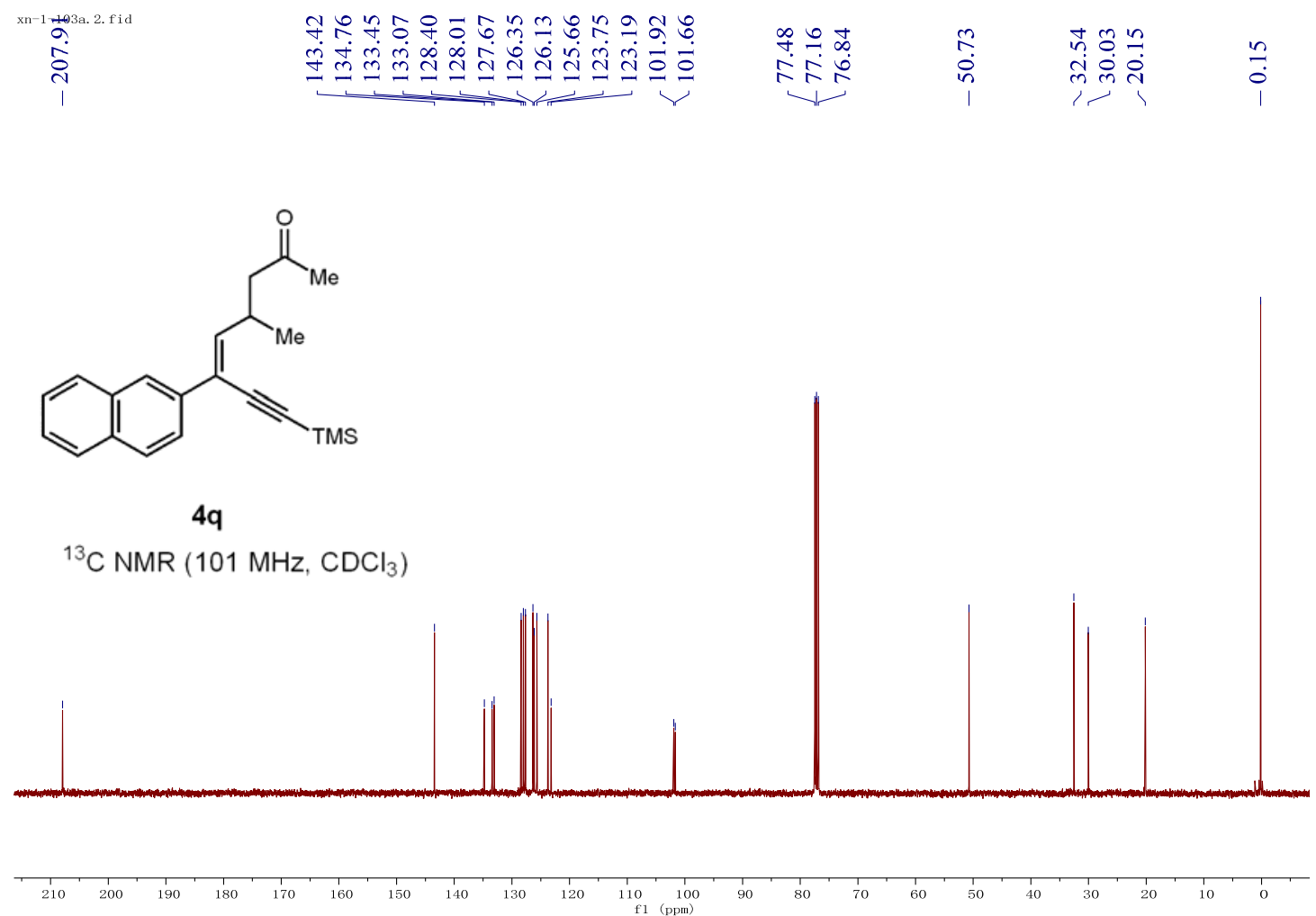

tert-Butyl-(Z)-5-(5-methyl-7-oxo-1-(trimethylsilyl)oct-3-en-1-yn-3-yl)-1 H-indole-1carboxylate (4r)

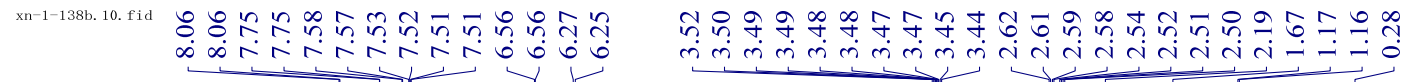

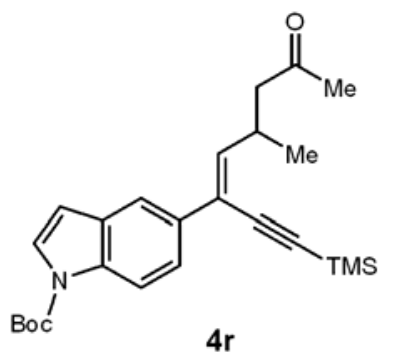

${ }^{1} \mathrm{H}$ NMR $\left(600 \mathrm{MHz}, \mathrm{CDCl}_{3}\right)$

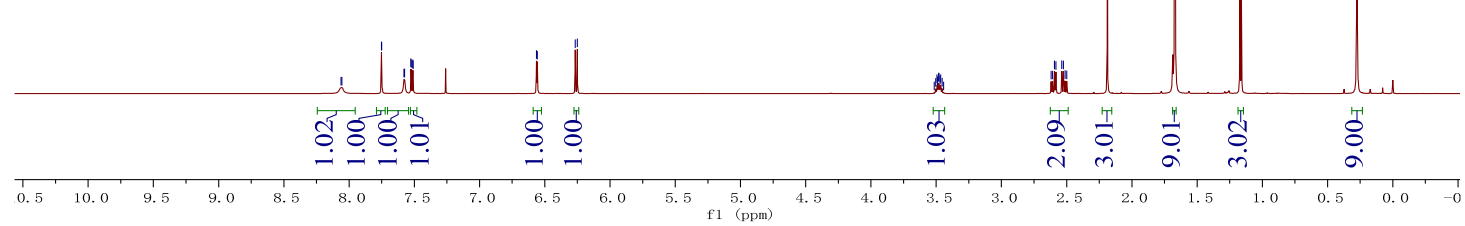




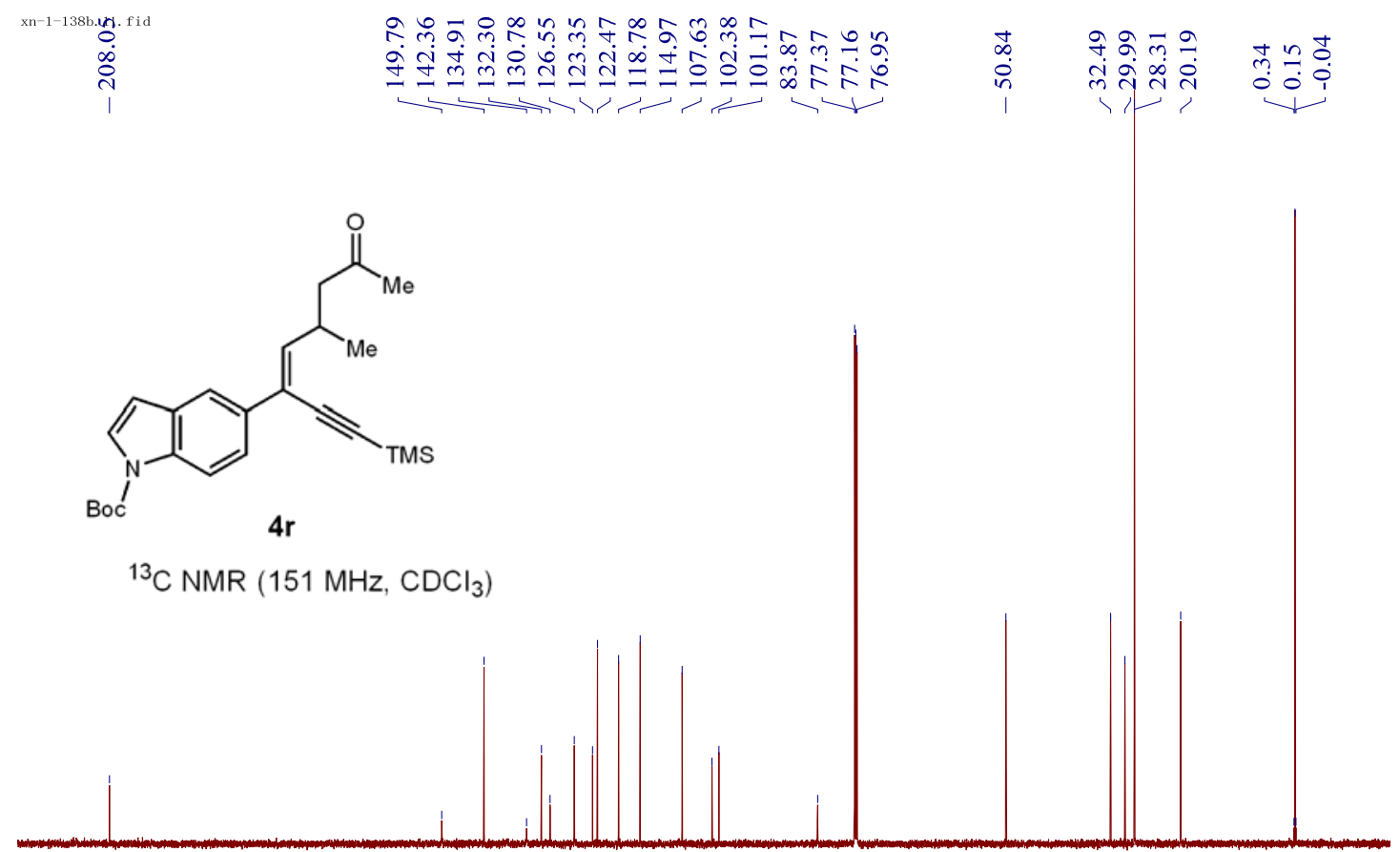

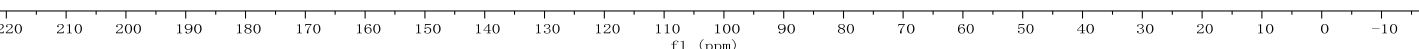

(E)-4-Methyl-6-(thiophen-2-yl)-8-(trimethylsilyl)oct-5-en-7-yn-2-one (4s)

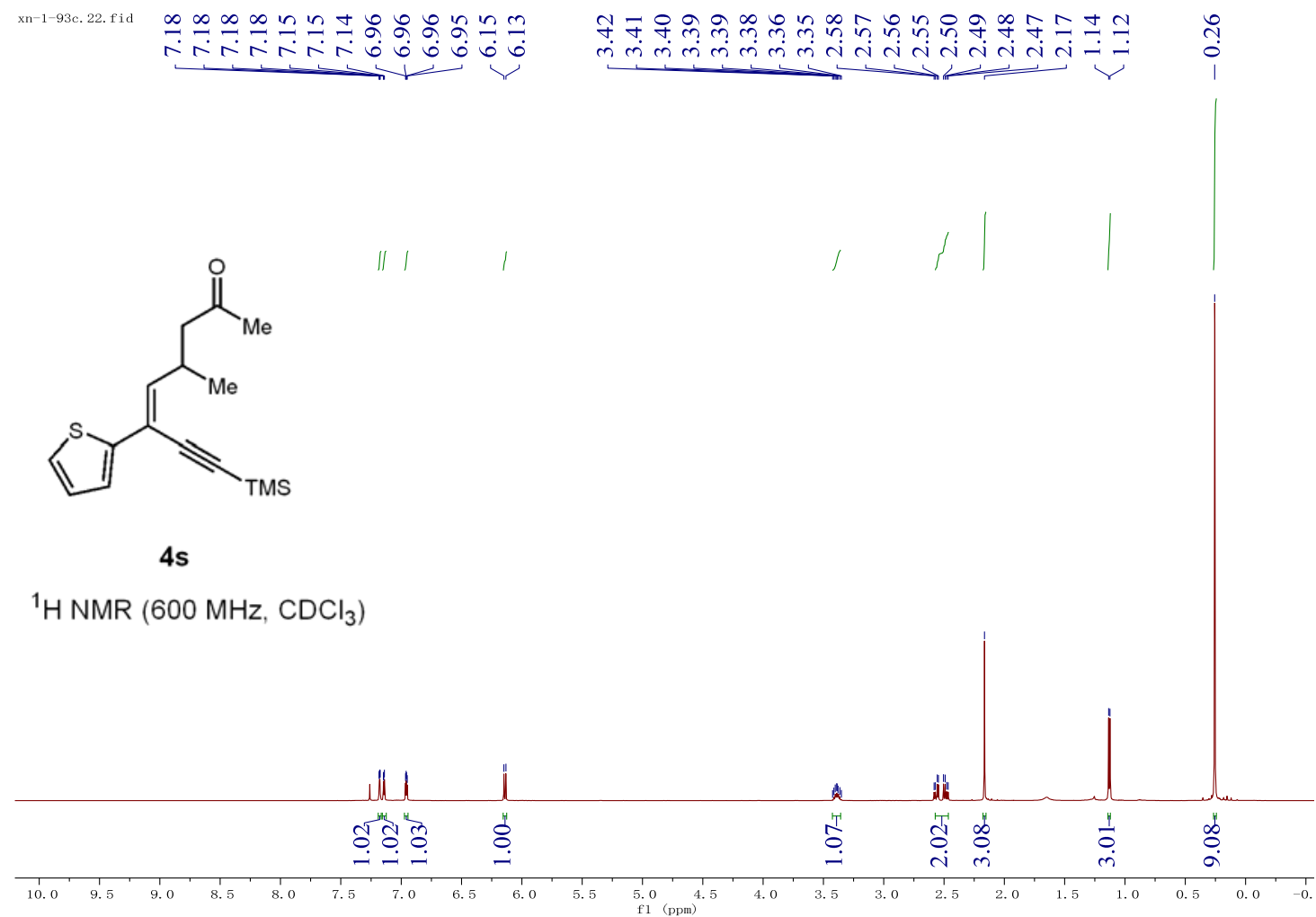




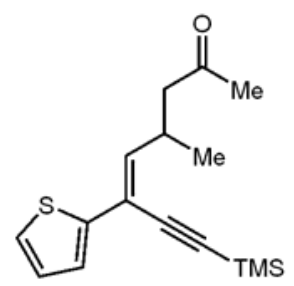

4s
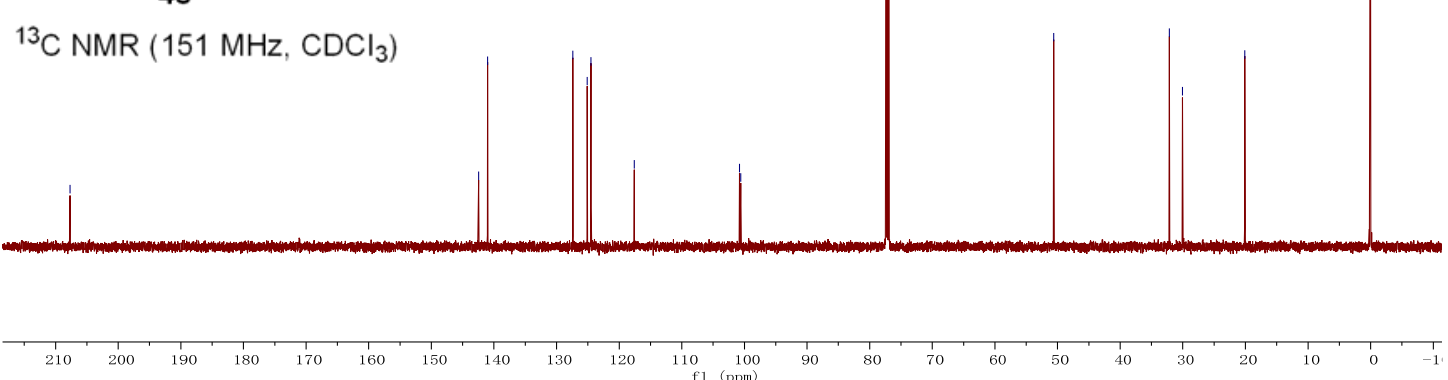

\section{(Z)-5-Methyl-7-phenyl-9-(trimethylsilyl)non-6-en-8-yn-3-one (5a)}

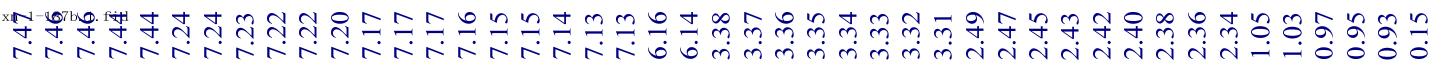<smiles>CC#C/C(=C/C(C)CC(=O)CC)c1ccccc1</smiles>

$5 a$

${ }^{1} \mathrm{H}$ NMR $\left(400 \mathrm{MHz}, \mathrm{CDCl}_{3}\right)$

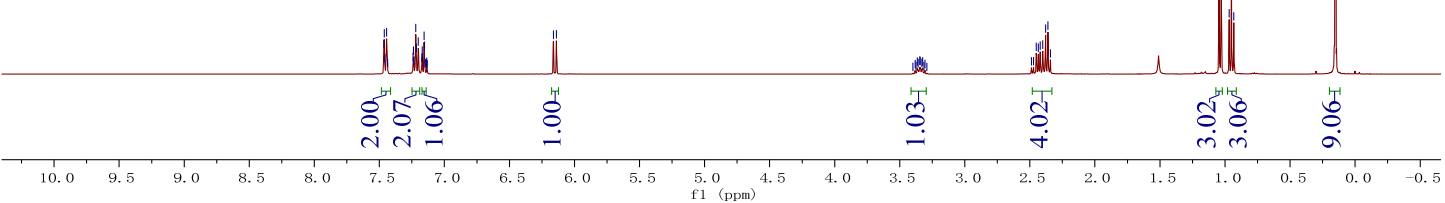



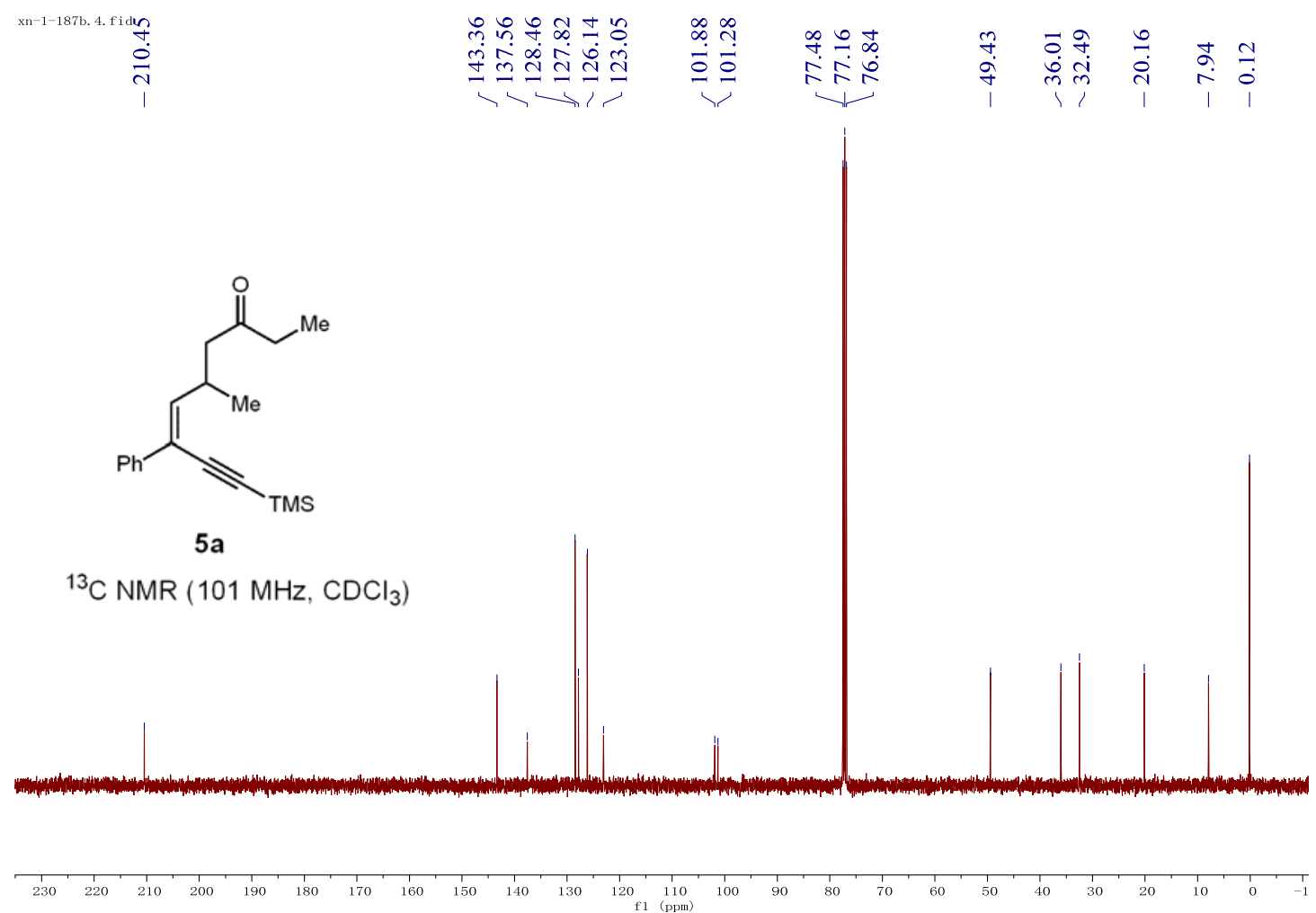

\section{(Z)-6-Phenyl-4-propyl-8-(trimethylsilyl)oct-5-en-7-yn-2-one (5b)}

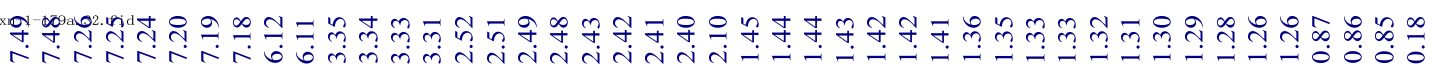<smiles>CC#C/C(=C/C(CCC)CC(N)=O)c1ccccc1</smiles>

$5 \mathrm{~b}$

${ }^{1} \mathrm{H} \mathrm{NMR}\left(600 \mathrm{MHz}, \mathrm{CDCl}_{3}\right)$

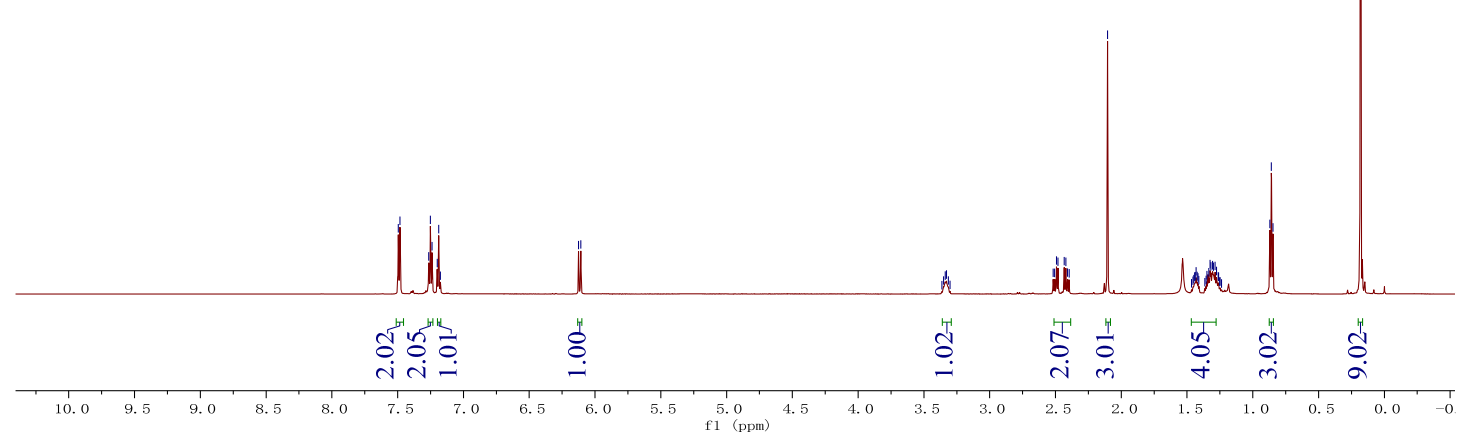



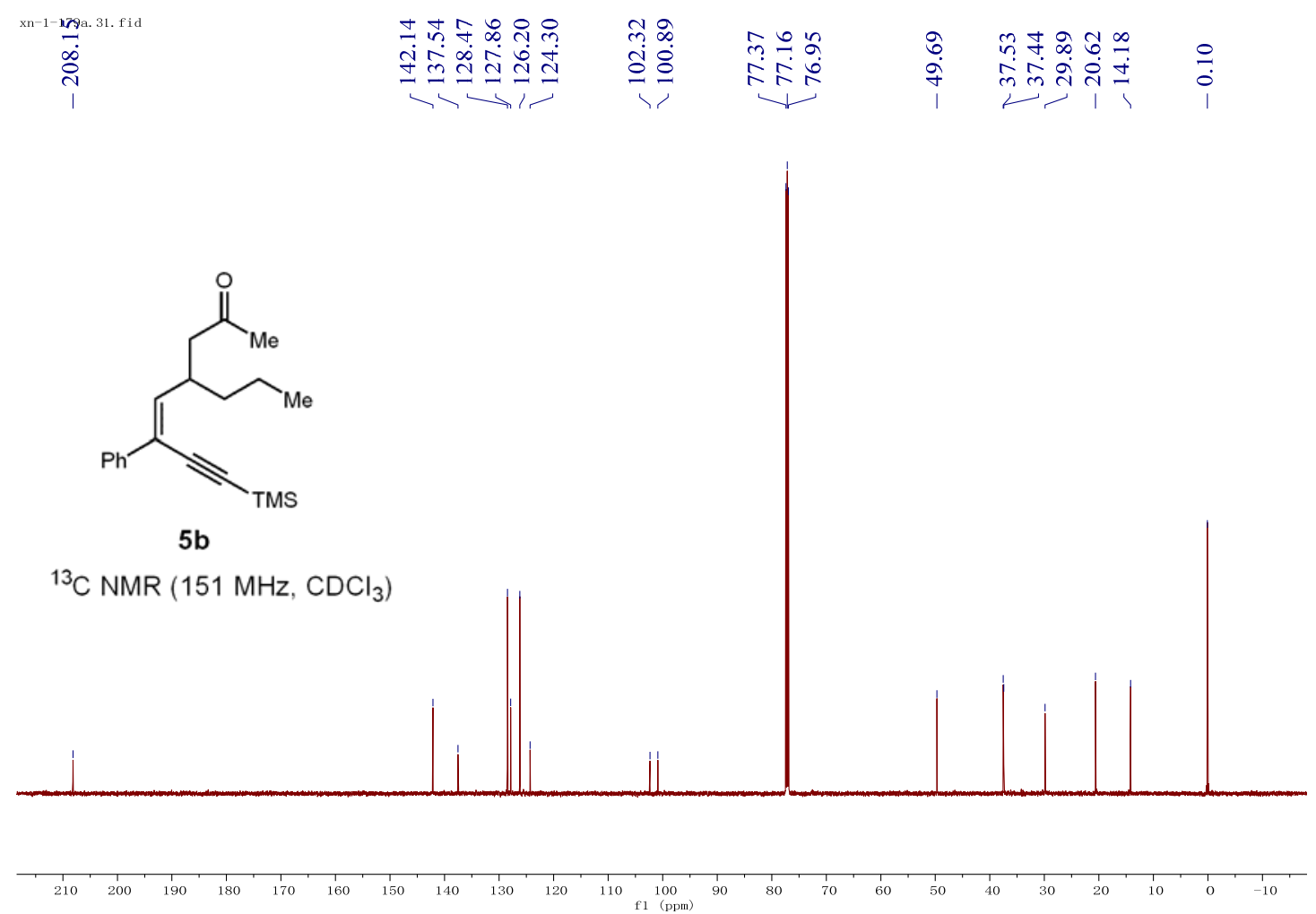

\section{(Z)-6-Phenyl-8-(trimethylsilyl)oct-5-en-7-yn-2-one (5c)}

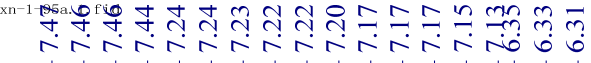
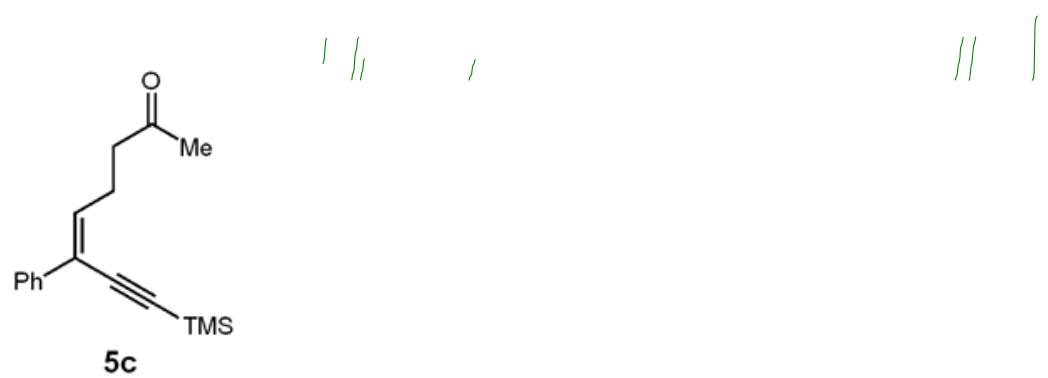

${ }^{1} \mathrm{H} \mathrm{NMR}\left(600 \mathrm{MHz}, \mathrm{CDCl}_{3}\right)$

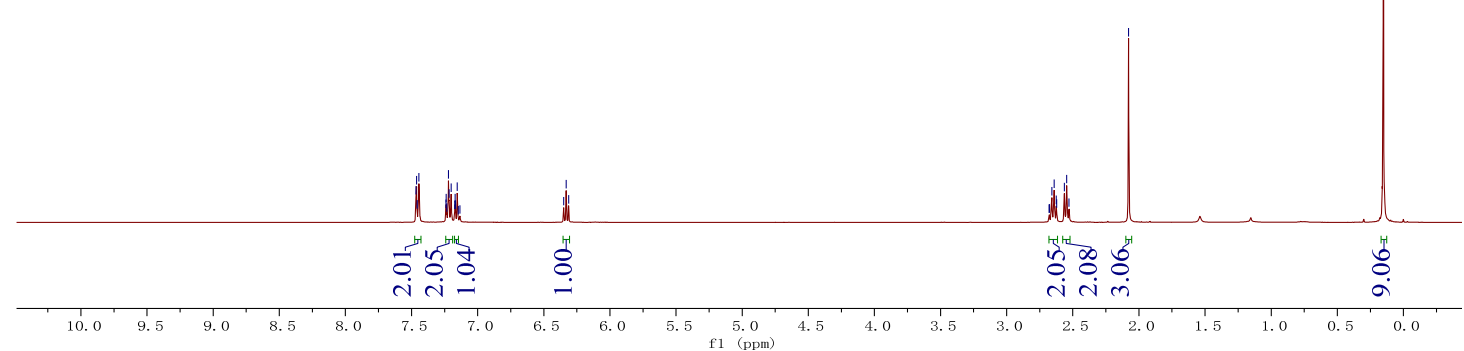




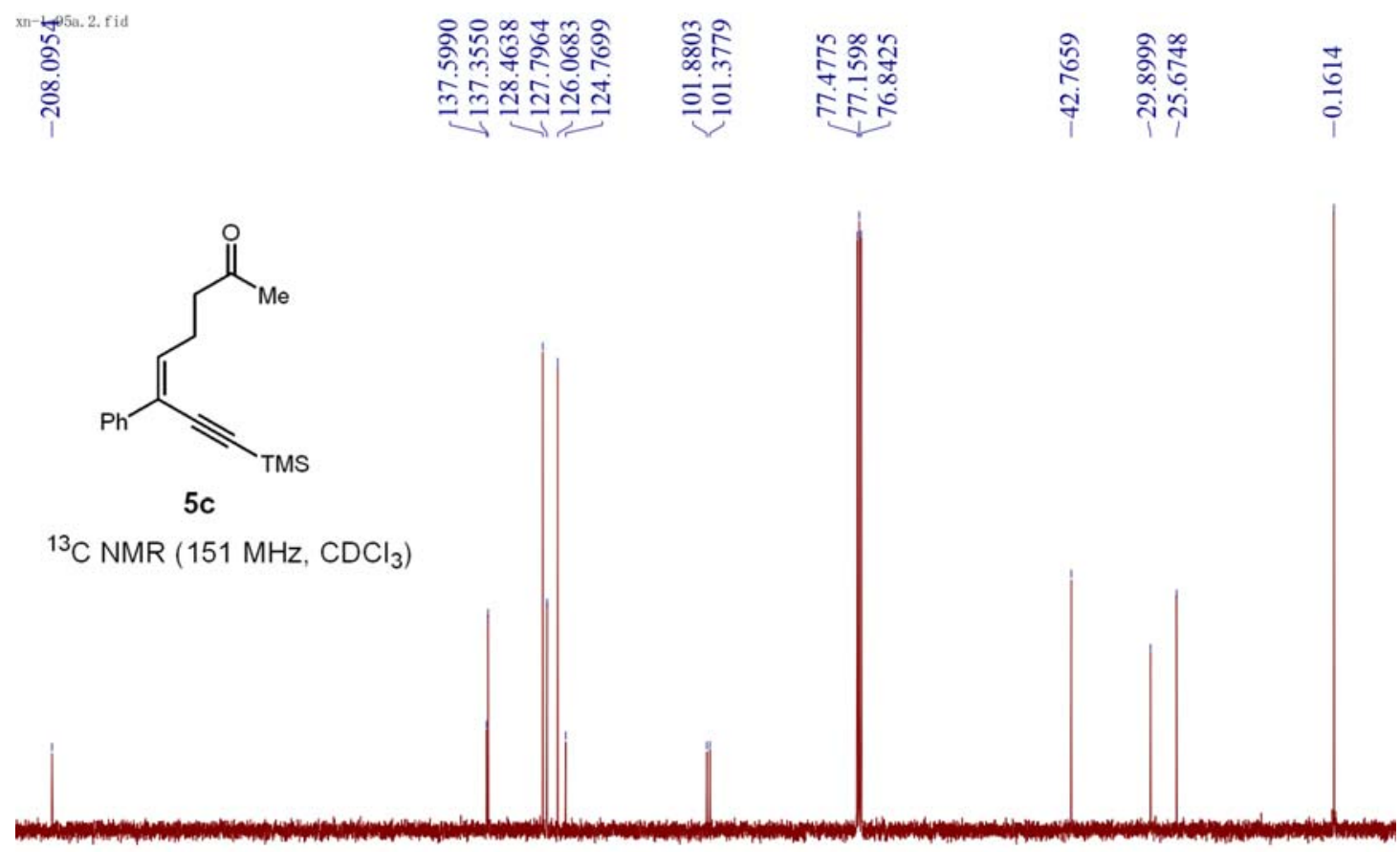

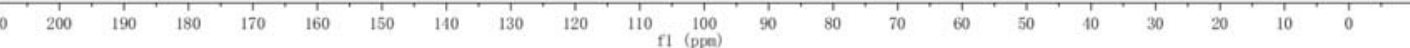

(Z)-7-Phenyl-9-(trimethylsilyl)non-6-en-8-yn-3-one (5d)

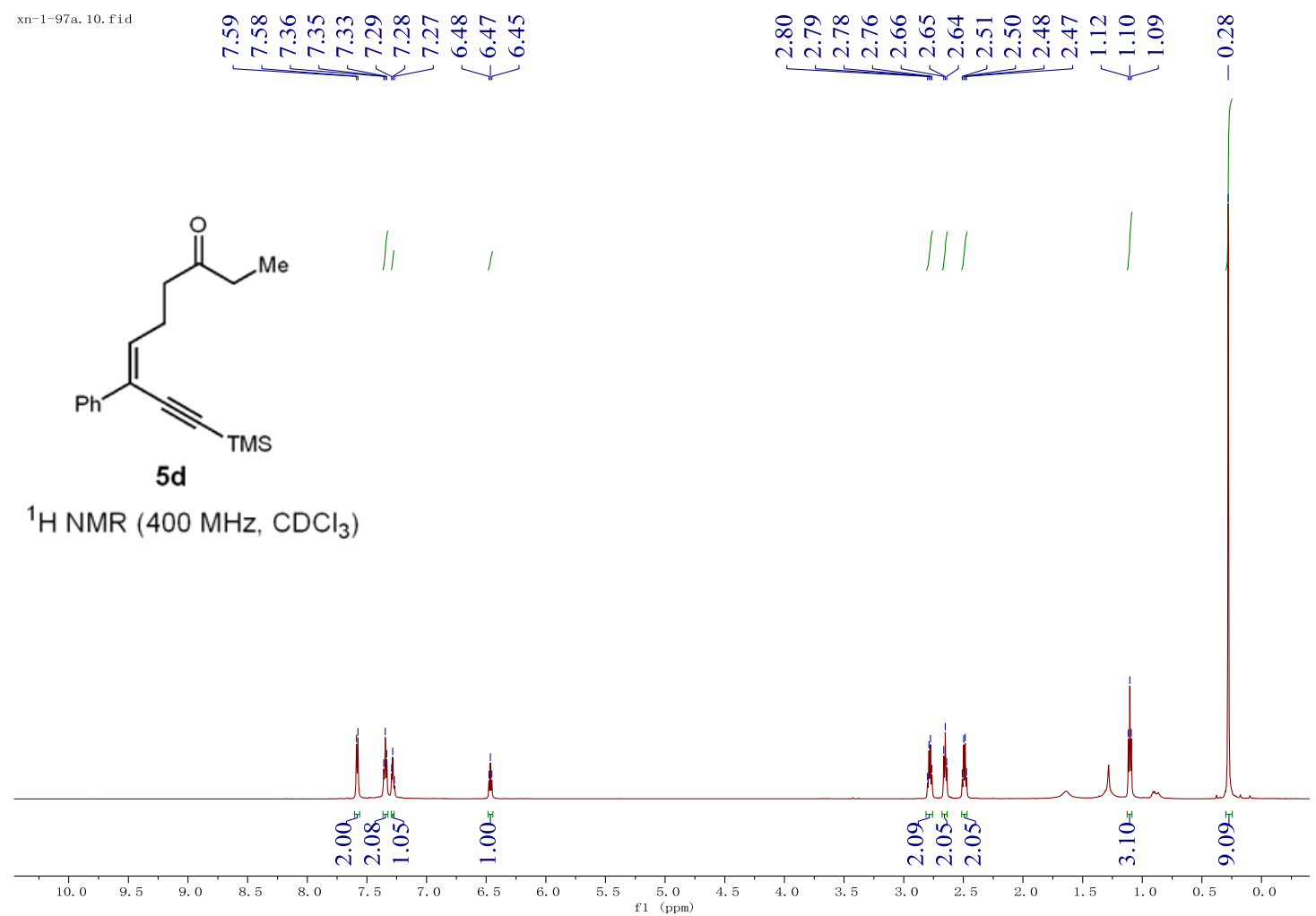




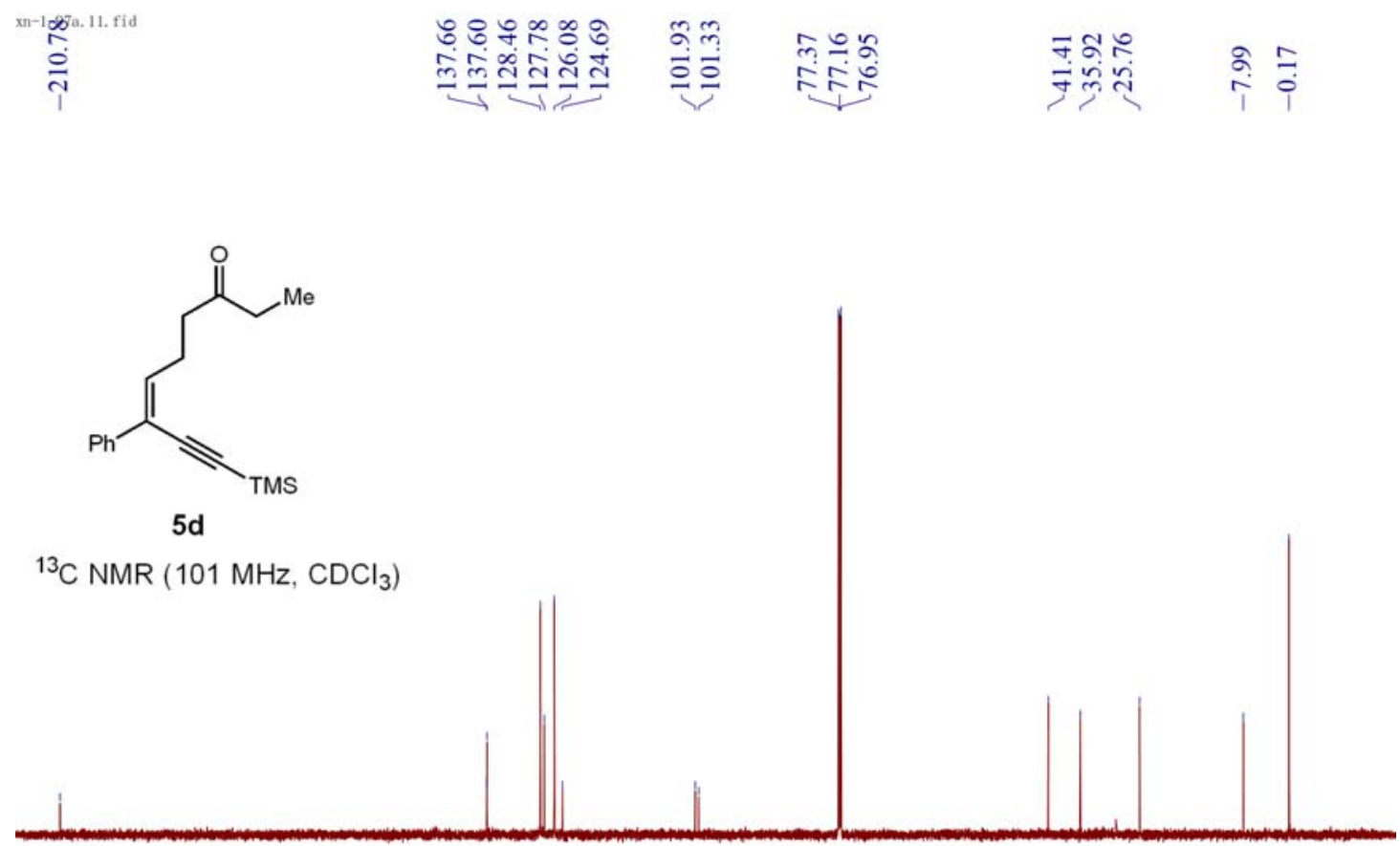

$\begin{array}{lllllllllllllllllllllll}210 & 200 & 190 & 180 & 170 & 160 & 150 & 140 & 130 & 120 & 110 & 100 & 90 & 80 & 70 & 60 & 50 & 40 & 30 & 20 & 10 & 0 & -10\end{array}$

\section{(Z)-3-(2-Phenyl-4-(trimethylsilyl)but-1-en-3-yn-1-yl)cyclopentan-1-one (5e)}

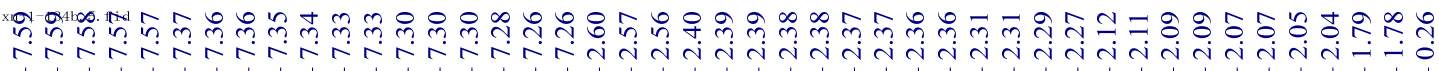

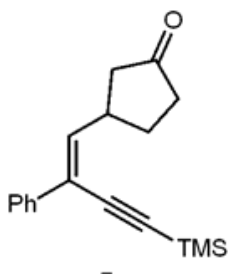

$5 e$

${ }^{1} \mathrm{H}$ NMR $\left(400 \mathrm{MHz}, \mathrm{CDCl}_{3}\right)$

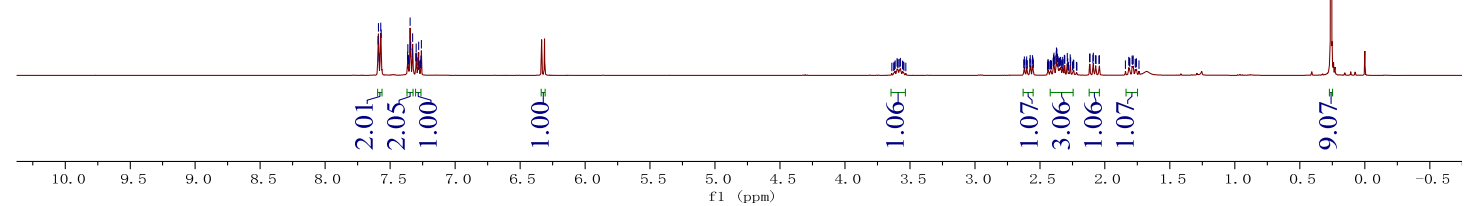




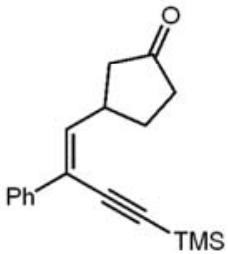

$5 e$

${ }^{13} \mathrm{C} \mathrm{NMR}\left(101 \mathrm{MHz}, \mathrm{CDCl}_{3}\right)$

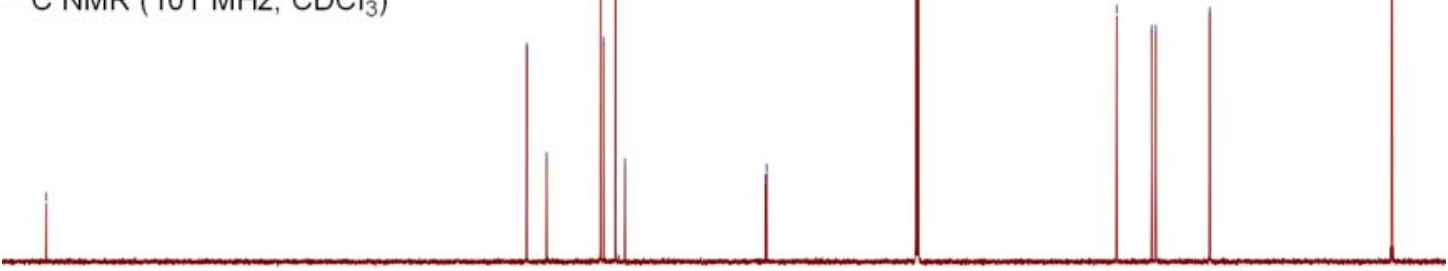

$220 \quad 210 \quad 200$

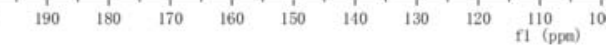

(Z)-3-(2-Phenyl-4-(trimethylsilyl)but-1-en-3-yn-1-yl)cyclohexan-1-one (5f)

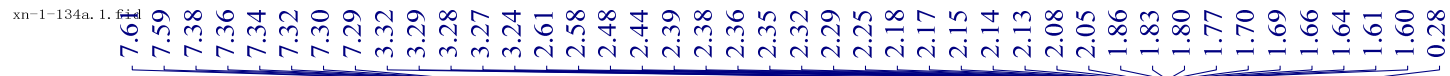<smiles>N#CC(=CC1CCCC(=O)C1)c1ccccc1</smiles>

$5 f$

${ }^{1} \mathrm{H}$ NMR $\left(400 \mathrm{MHz}, \mathrm{CDCl}_{3}\right.$ )

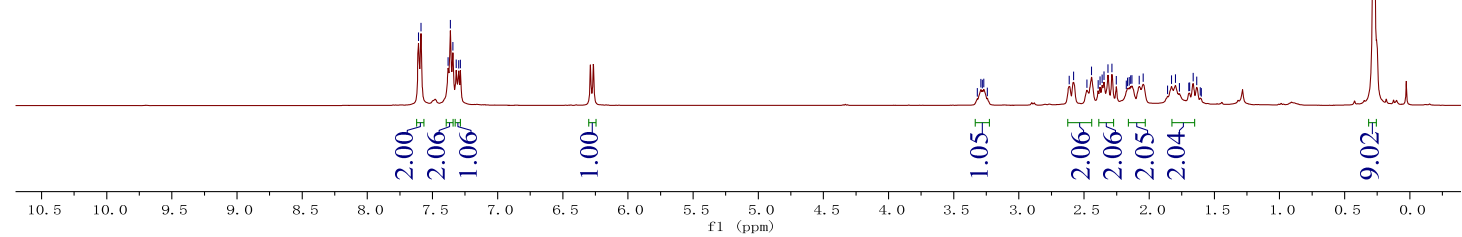




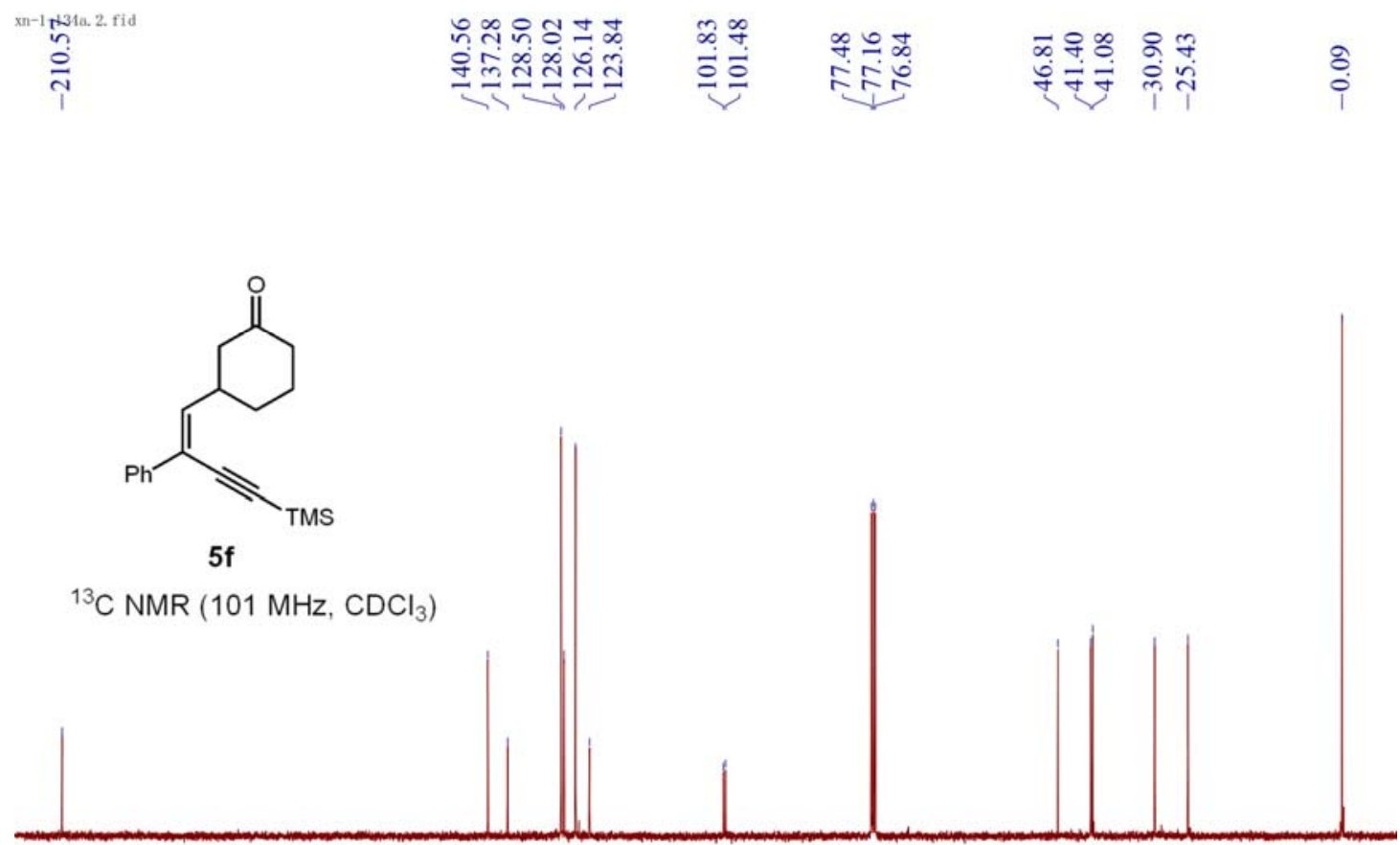

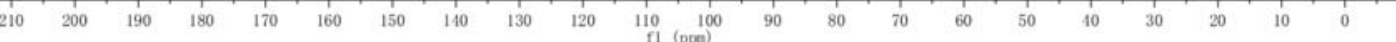

(Z)-3-(2-Phenyl-4-(trimethylsilyl)but-1-en-3-yn-1-yl)cycloheptan-1-one (5g)

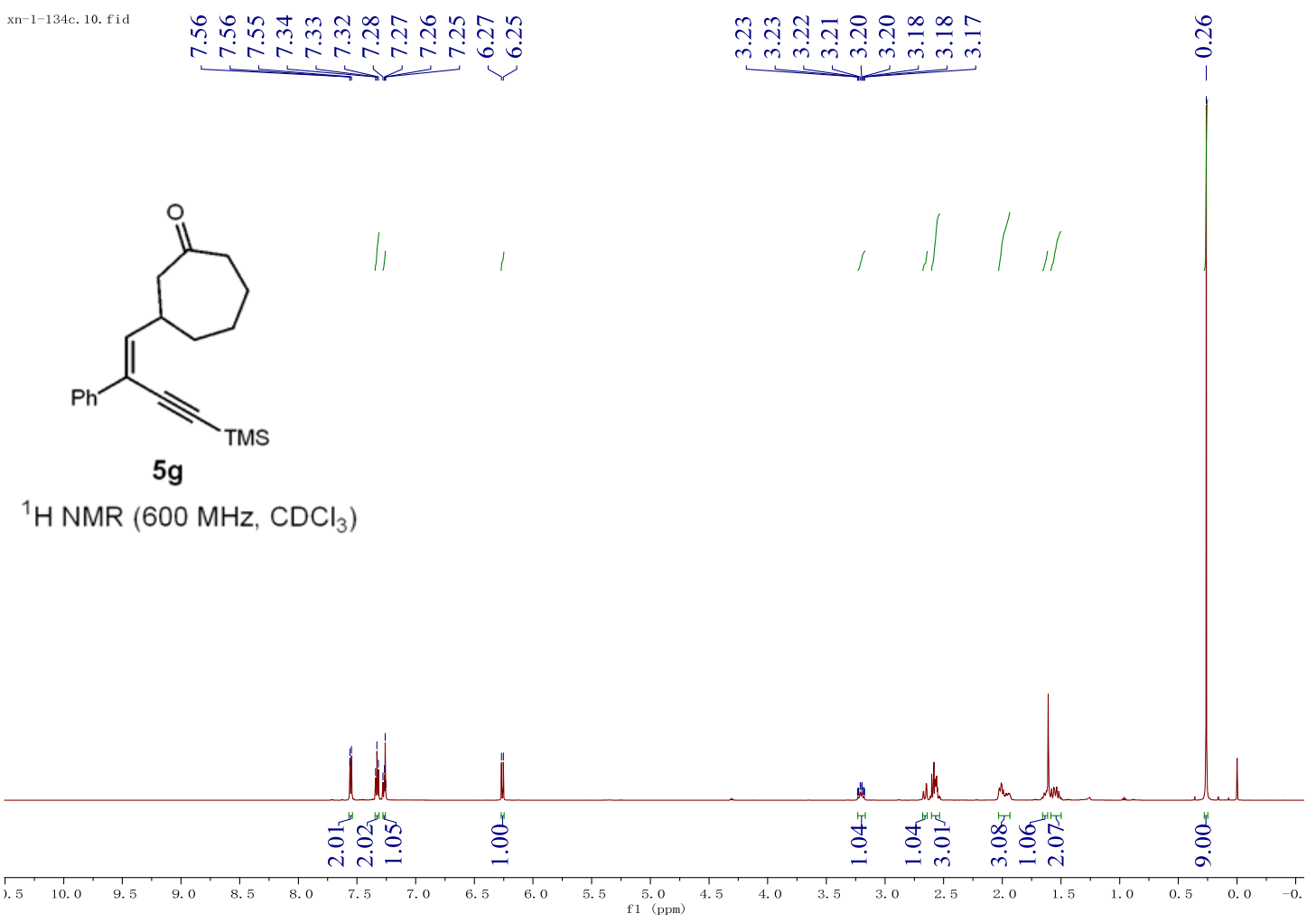



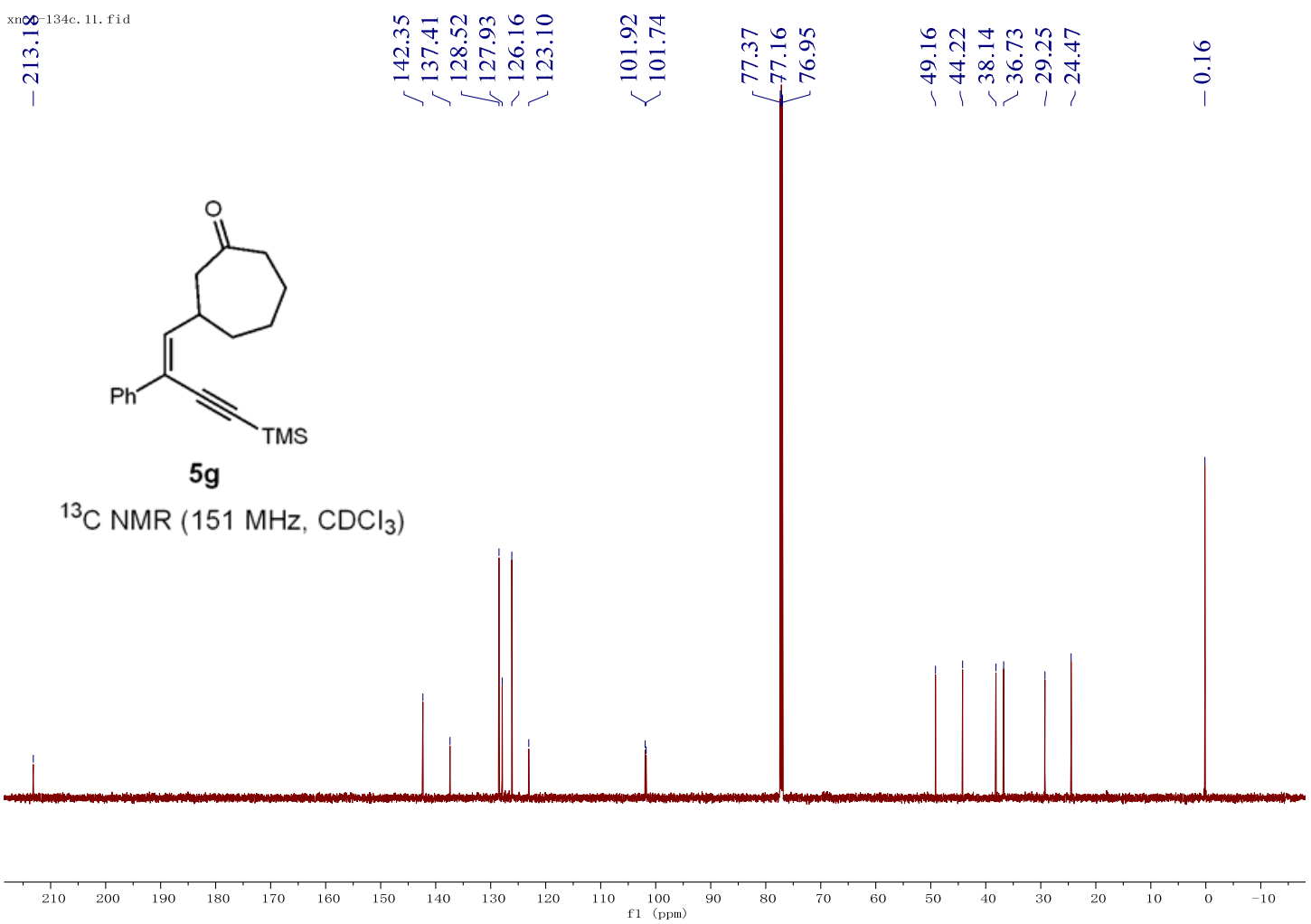

\section{(Z)-4,9,9-Trimethyl-6-phenyldec-5-en-7-yn-2-one (6a)}

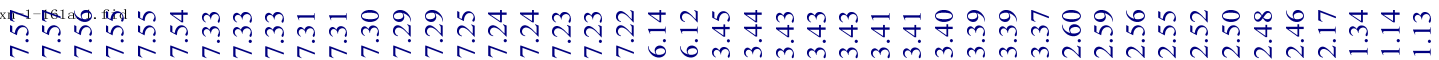

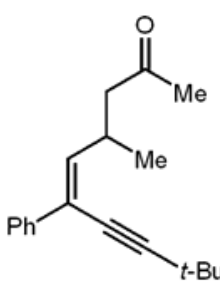

$6 a$

${ }^{1} \mathrm{H} \mathrm{NMR}\left(400 \mathrm{MHz}, \mathrm{CDCl}_{3}\right)$

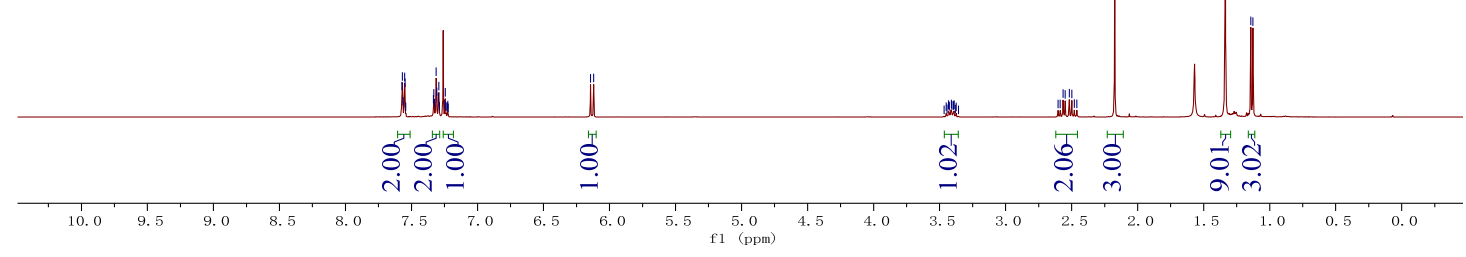



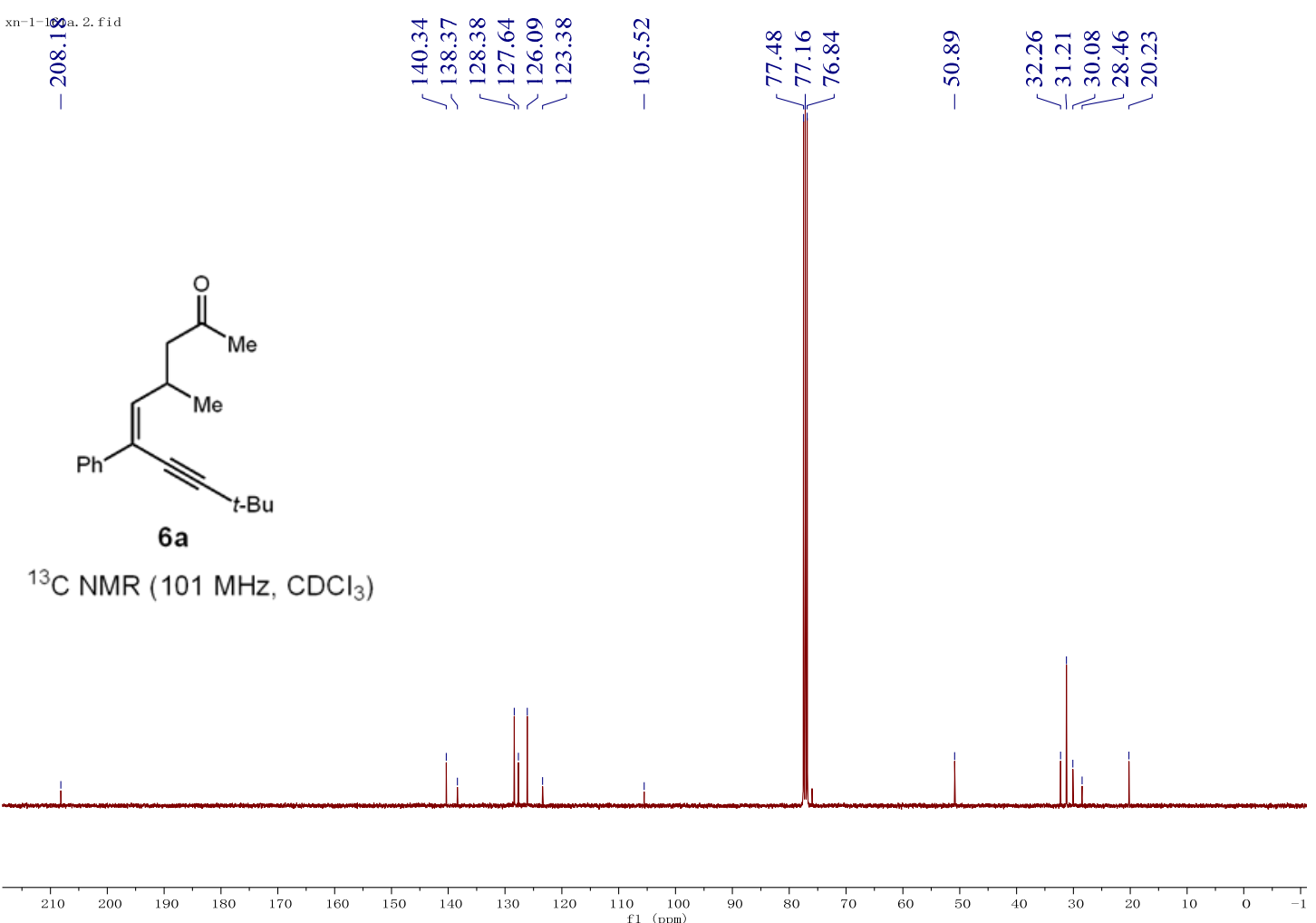

\section{(Z)-4,9,9-Trimethyl-6-phenyldec-5-en-7-yn-2-one (6b)}

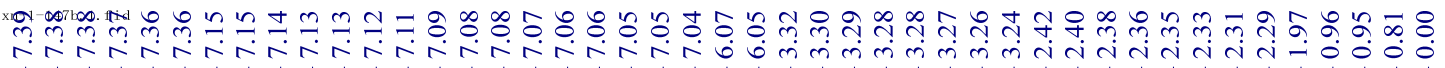

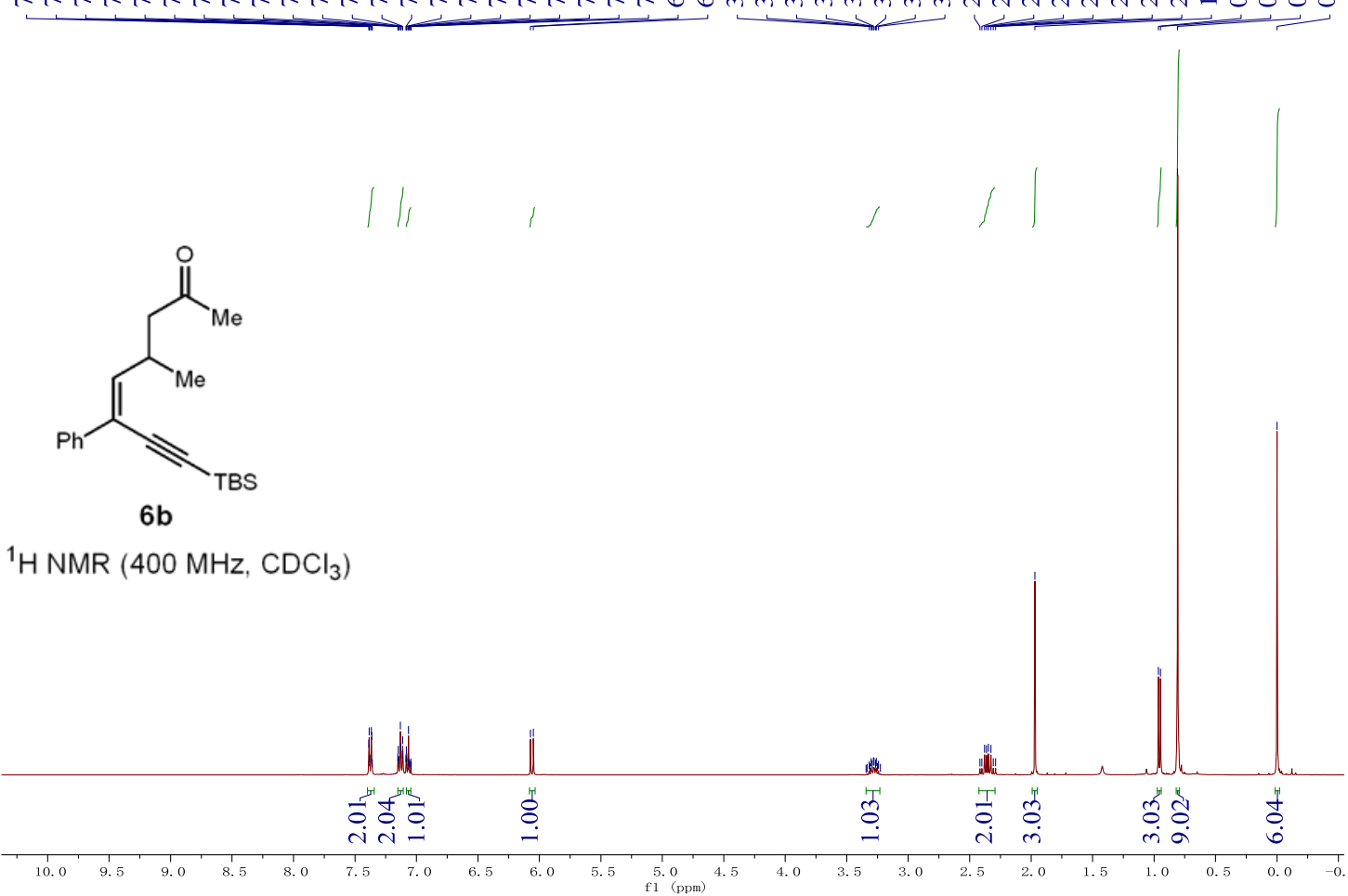



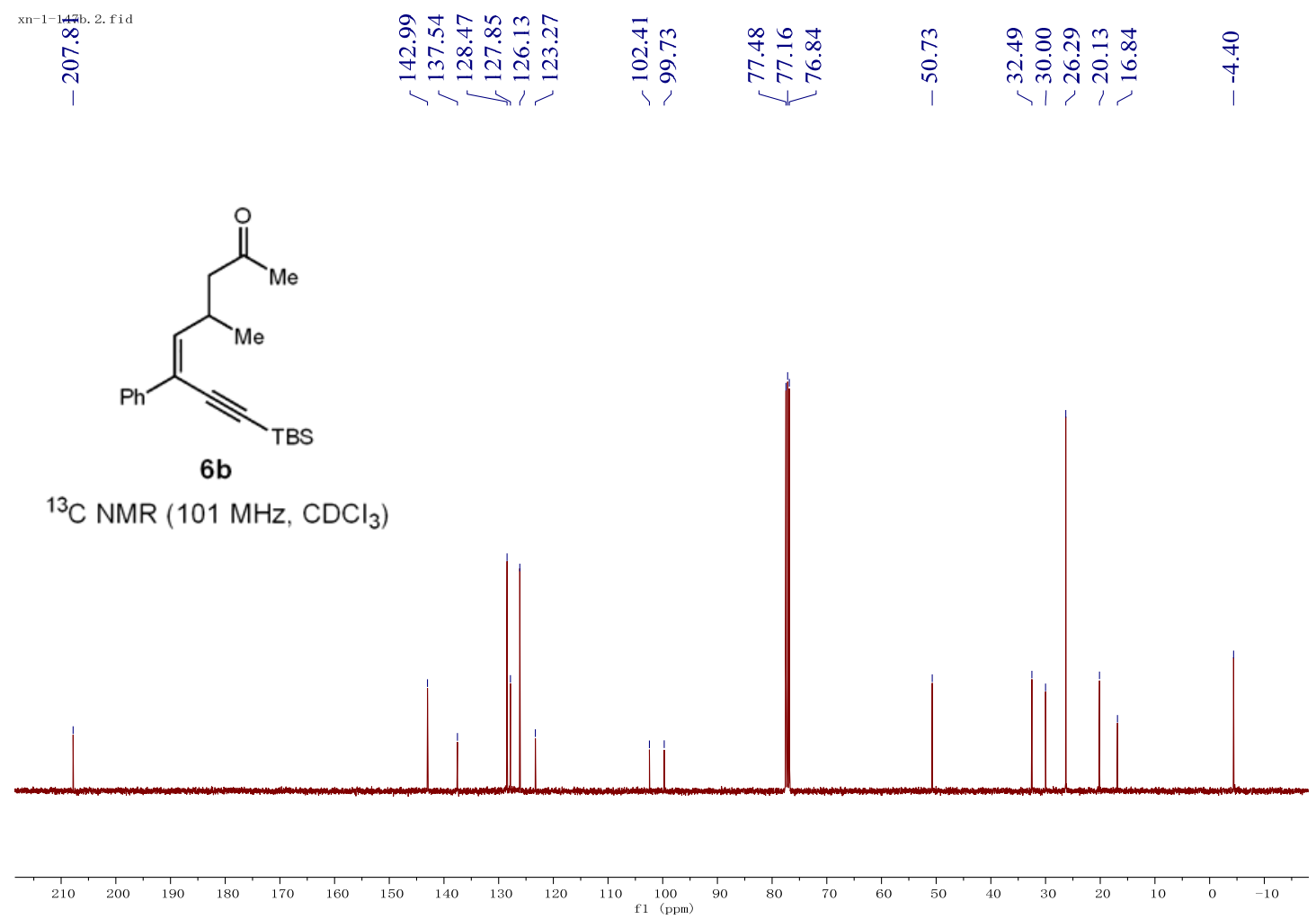

\section{(Z)-4-Methyl-6-phenyldodec-5-en-7-yn-2-one (6c)}

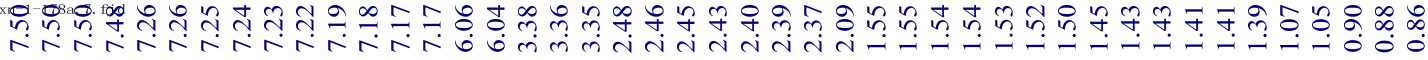

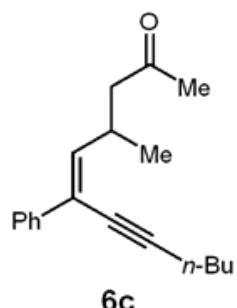

$6 \mathrm{c}$

${ }^{1} \mathrm{H}$ NMR $\left(400 \mathrm{MHz}, \mathrm{CDCl}_{3}\right)$

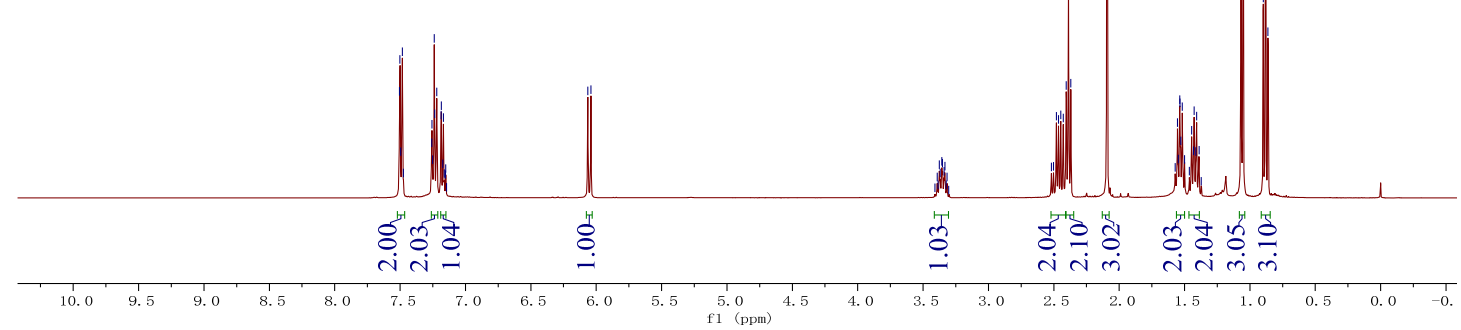



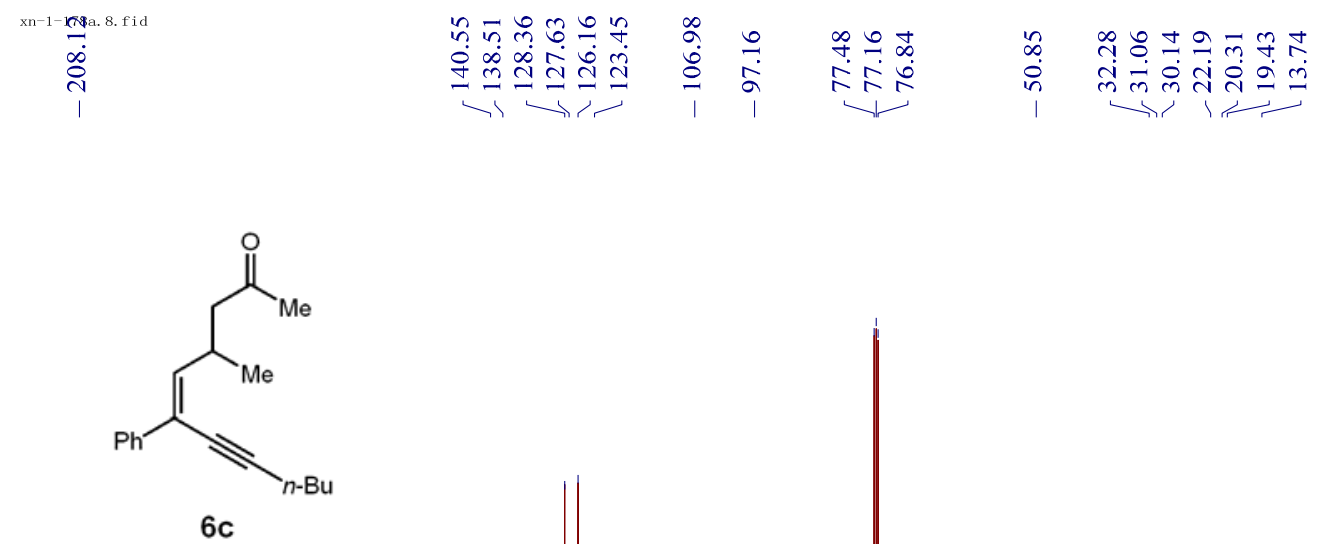

${ }^{13} \mathrm{C}$ NMR $\left(101 \mathrm{MHz}, \mathrm{CDCl}_{3}\right)$

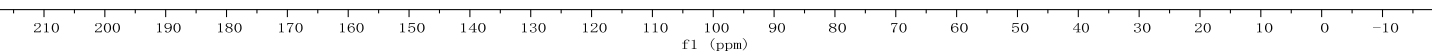

\section{(Z)-4-Methyl-6-phenyl-8-(triethylsilyl)oct-5-en-7-yn-2-one (6d)}

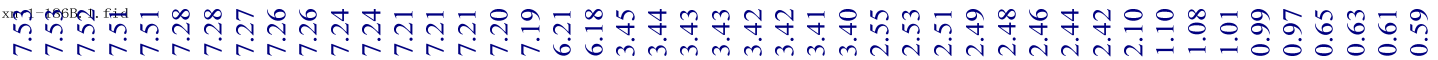<smiles>CCCCCCCC#CC#C/C(=C/C(C)CC(C)=O)c1ccccc1</smiles>

$6 d$

${ }^{1} \mathrm{H} \mathrm{NMR}\left(600 \mathrm{MHz}, \mathrm{CDCl}_{3}\right)$

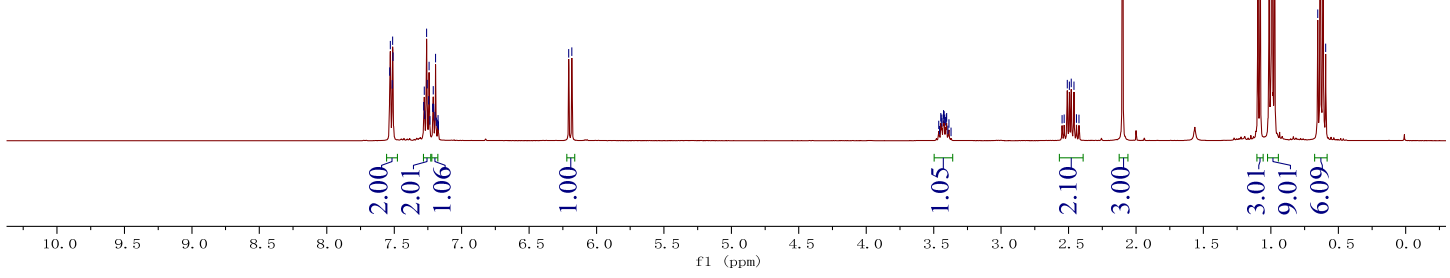




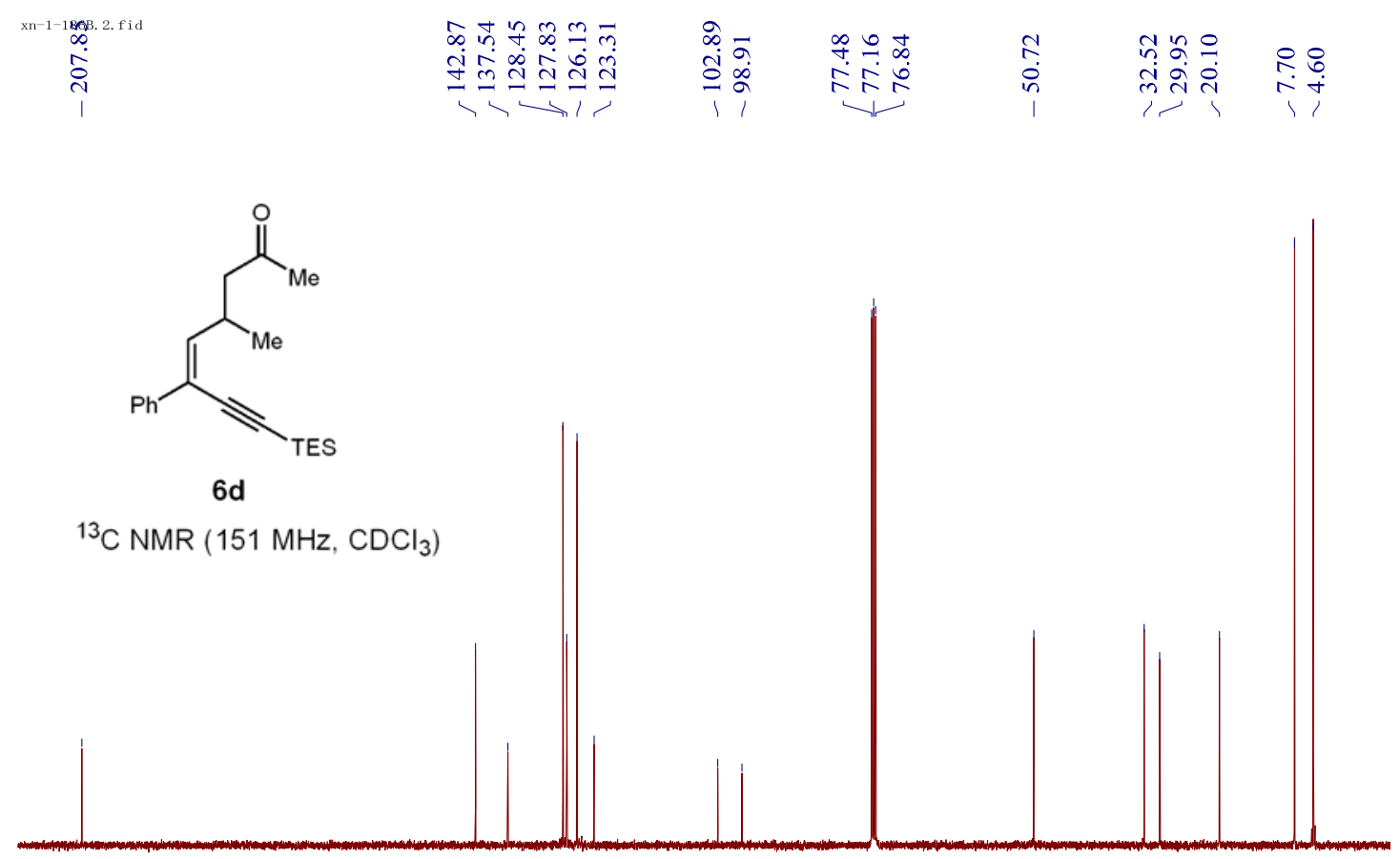

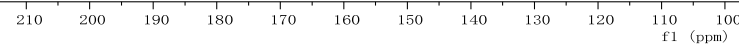

\section{(Z)-6-(4-Chlorophenyl)-4-methyl-8-phenyloct-5-en-7-yn-2-one (6e)}

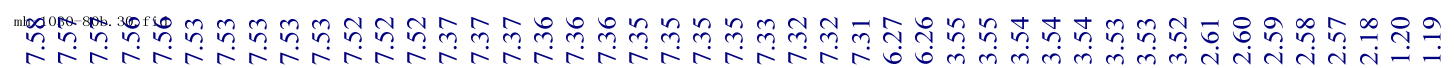<smiles>CC(=O)CC(C)/C=C(\C#Cc1ccccc1)c1ccc(Cl)cc1</smiles>

${ }^{1} \mathrm{H}$ NMR $\left(600 \mathrm{MHz}, \mathrm{CDCl}_{3}\right)$

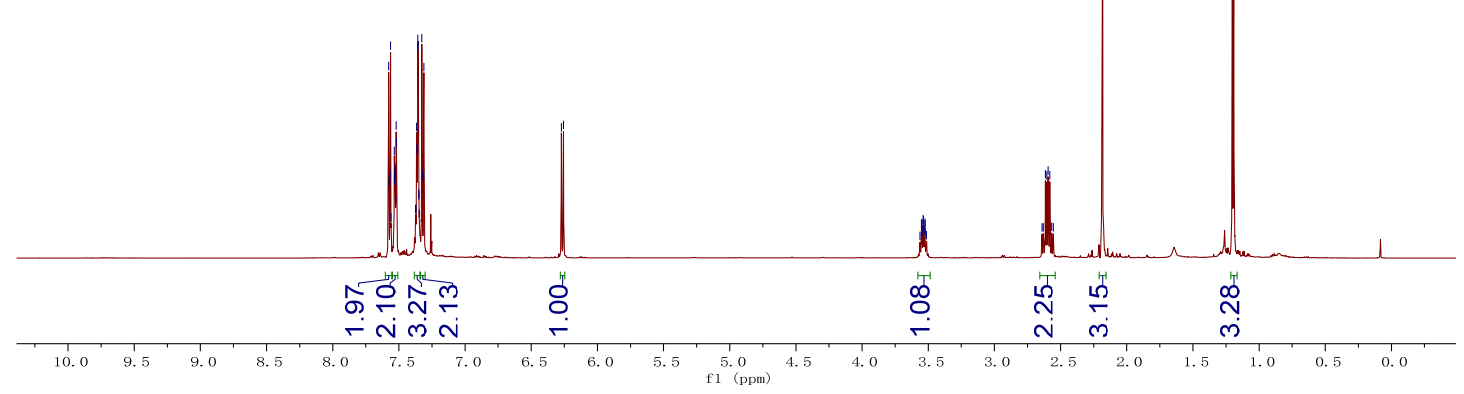




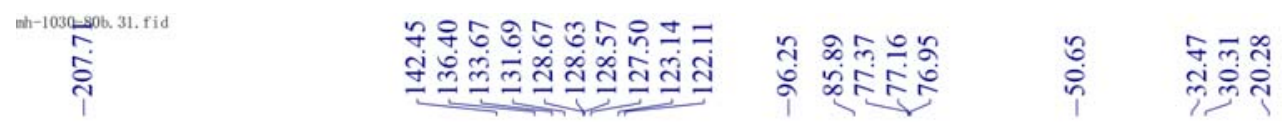<smiles>CC(=O)CC(C)/C=C(\C#Cc1ccccc1)c1ccc(Cl)cc1</smiles>

$6 e$

${ }^{1} \mathrm{H} \mathrm{NMR}\left(600 \mathrm{MHz}, \mathrm{CDCl}_{3}\right)$
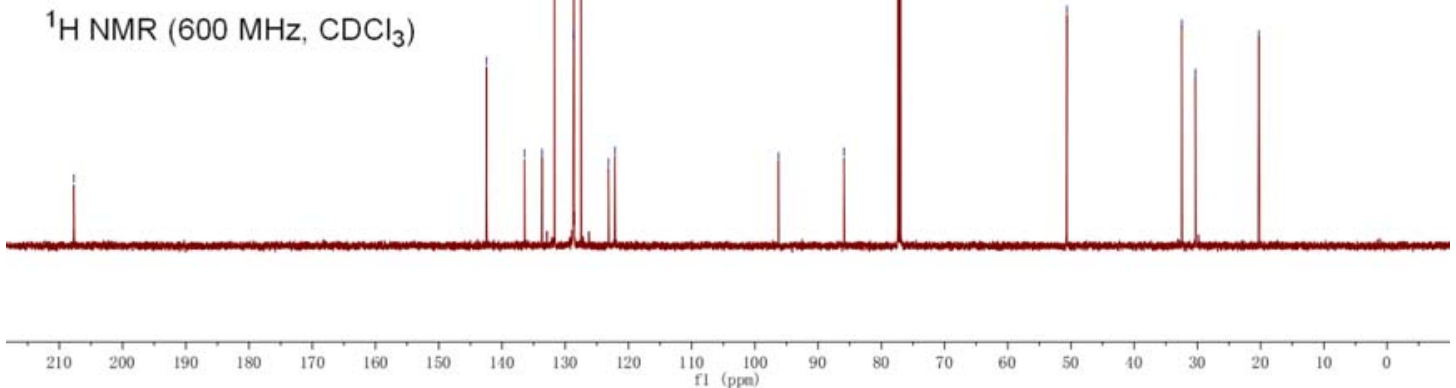

(Z)-6-(4-Bromophenyl)-4-methyl-8-phenyloct-5-en-7-yn-2-one (6f)

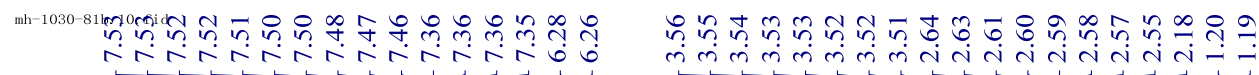<smiles>CC(=O)CC(C)/C=C(\C#Cc1ccccc1)c1ccc(Br)cc1</smiles>

$6 f$

${ }^{1} \mathrm{H} \mathrm{NMR}\left(600 \mathrm{MHz}, \mathrm{CDCl}_{3}\right)$

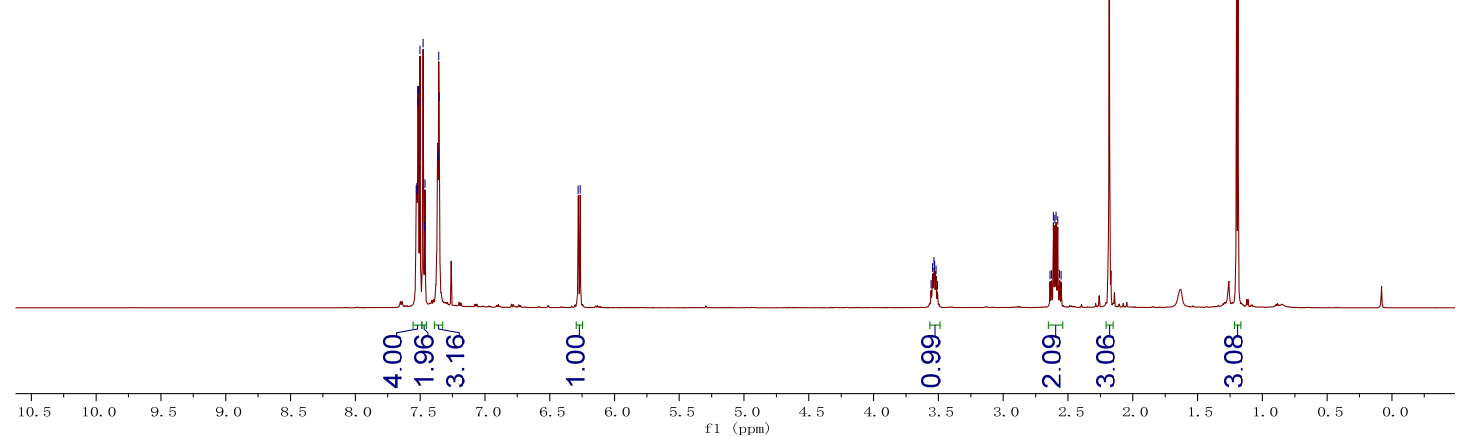



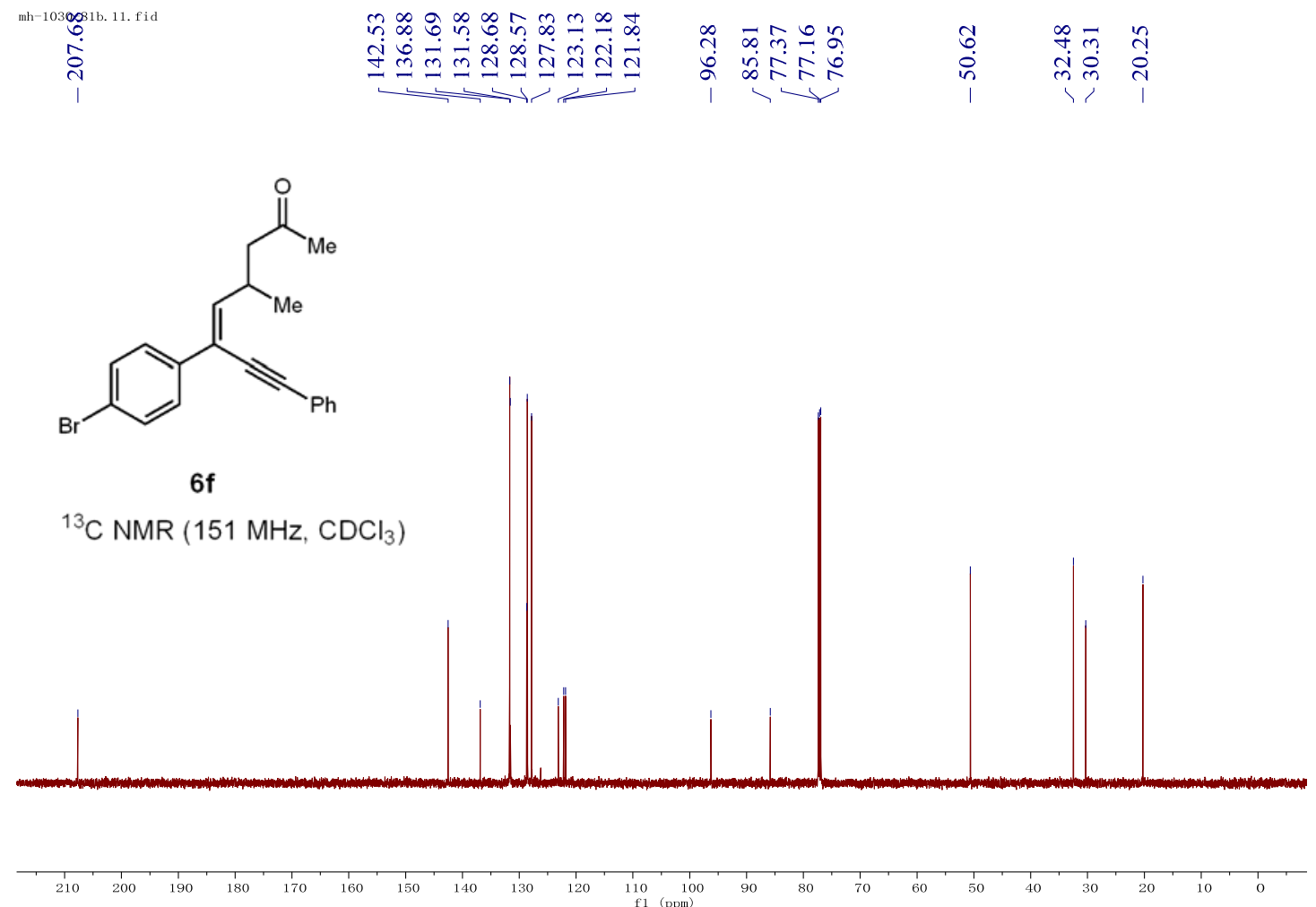

(Z)-6-(3-Fluorophenyl)-4-methyl-8-phenyloct-5-en-7-yn-2-one (6g)

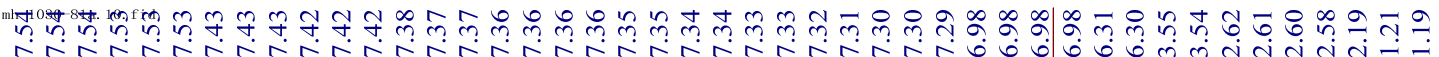<smiles>CC(=O)CC(C)C=C(C#Cc1ccccc1)c1cccc(F)c1</smiles>

$6 \mathrm{~g}$

${ }^{1} \mathrm{H}$ NMR $\left(600 \mathrm{MHz}, \mathrm{CDCl}_{3}\right)$

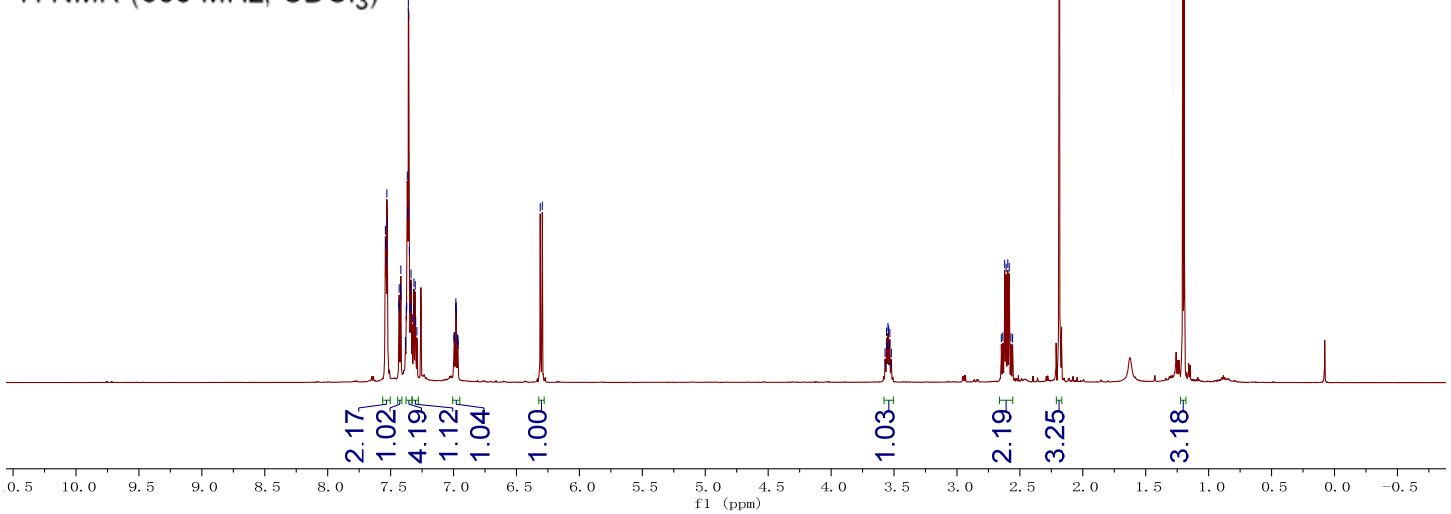


<smiles>CC(=O)CC(C)C=C(C#Cc1ccccc1)c1cccc(F)c1</smiles>

${ }^{19} \mathrm{~F} \mathrm{NMR} \mathrm{(376} \mathrm{MHz}, \mathrm{CDCl}_{3}$ )

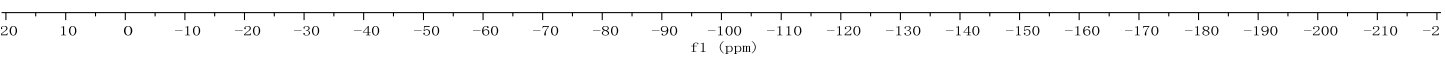

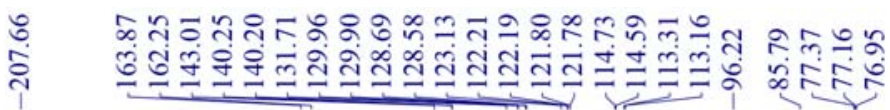<smiles>CC(=O)CC(C)C=C(C#Cc1ccccc1)c1cccc(F)c1</smiles>

$6 \mathrm{~g}$

$\left.{ }^{13} \mathrm{C} \mathrm{NMR} \mathrm{(151} \mathrm{MHz,} \mathrm{CDCl}_{3}\right)$

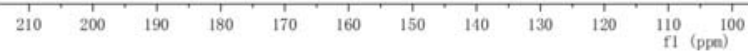


(Z)-4-Ethyl-6,8-diphenyloct-5-en-7-yn-2-one (7a)

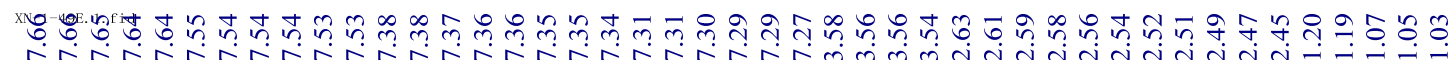

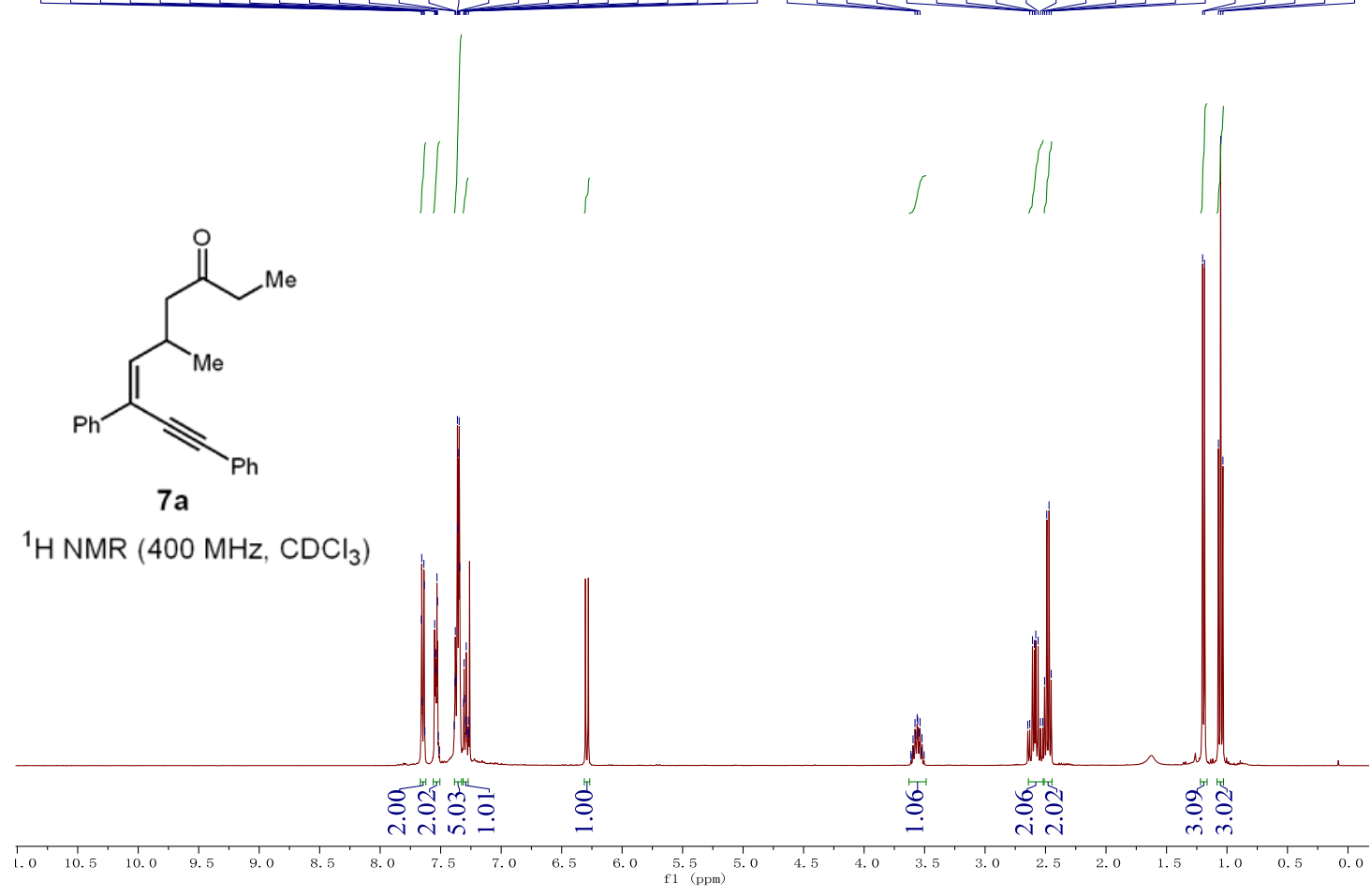

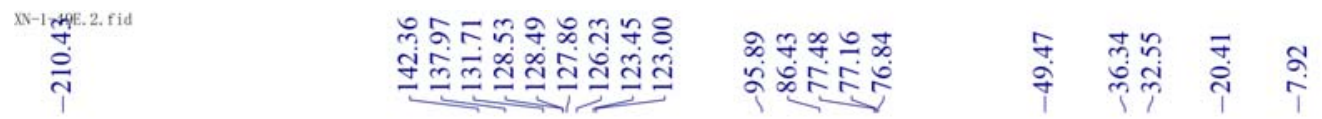<smiles>CCC(=O)CC(C)/C=C(/C#Cc1ccccc1)c1ccccc1</smiles>

7a

${ }^{13} \mathrm{C}$ NMR $\left(101 \mathrm{MHz}, \mathrm{CDCl}_{3}\right)$

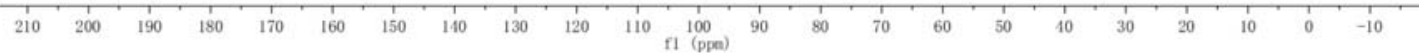


(Z)-4-Methyl-6,8-di-p-tolyloct-5-en-7-yn-2-one (7b)

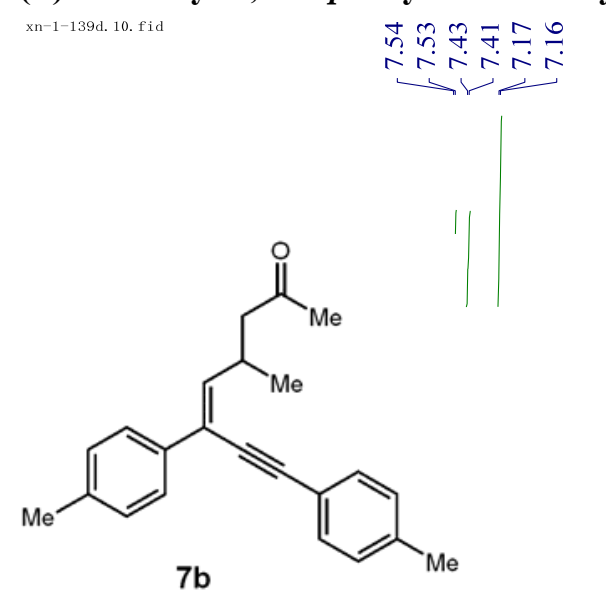

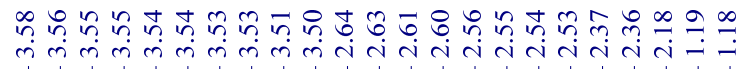

${ }^{1} \mathrm{H}$ NMR $\left(600 \mathrm{MHz}, \mathrm{CDCl}_{3}\right)$

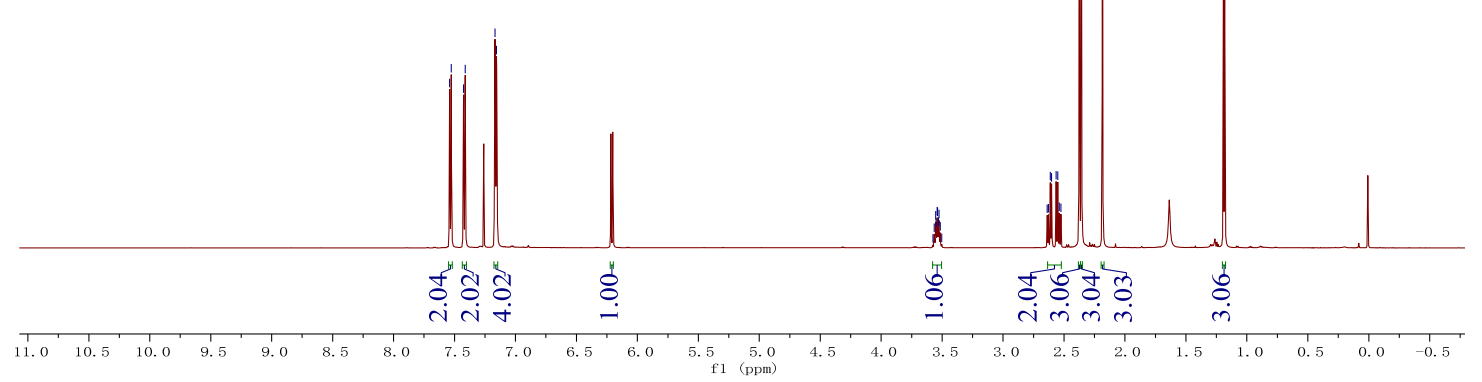

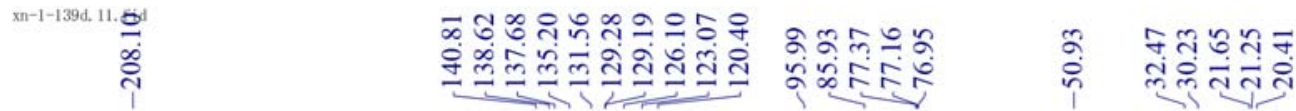<smiles>CC(=O)CC(C)/C=C(\C#Cc1ccc(C)cc1)c1ccc(C)cc1</smiles>

${ }^{13} \mathrm{C}$ NMR $\left(151 \mathrm{MHz}, \mathrm{CDCl}_{3}\right)$

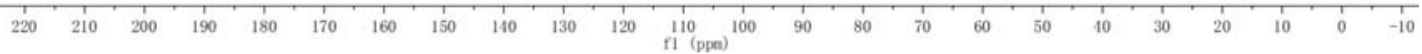




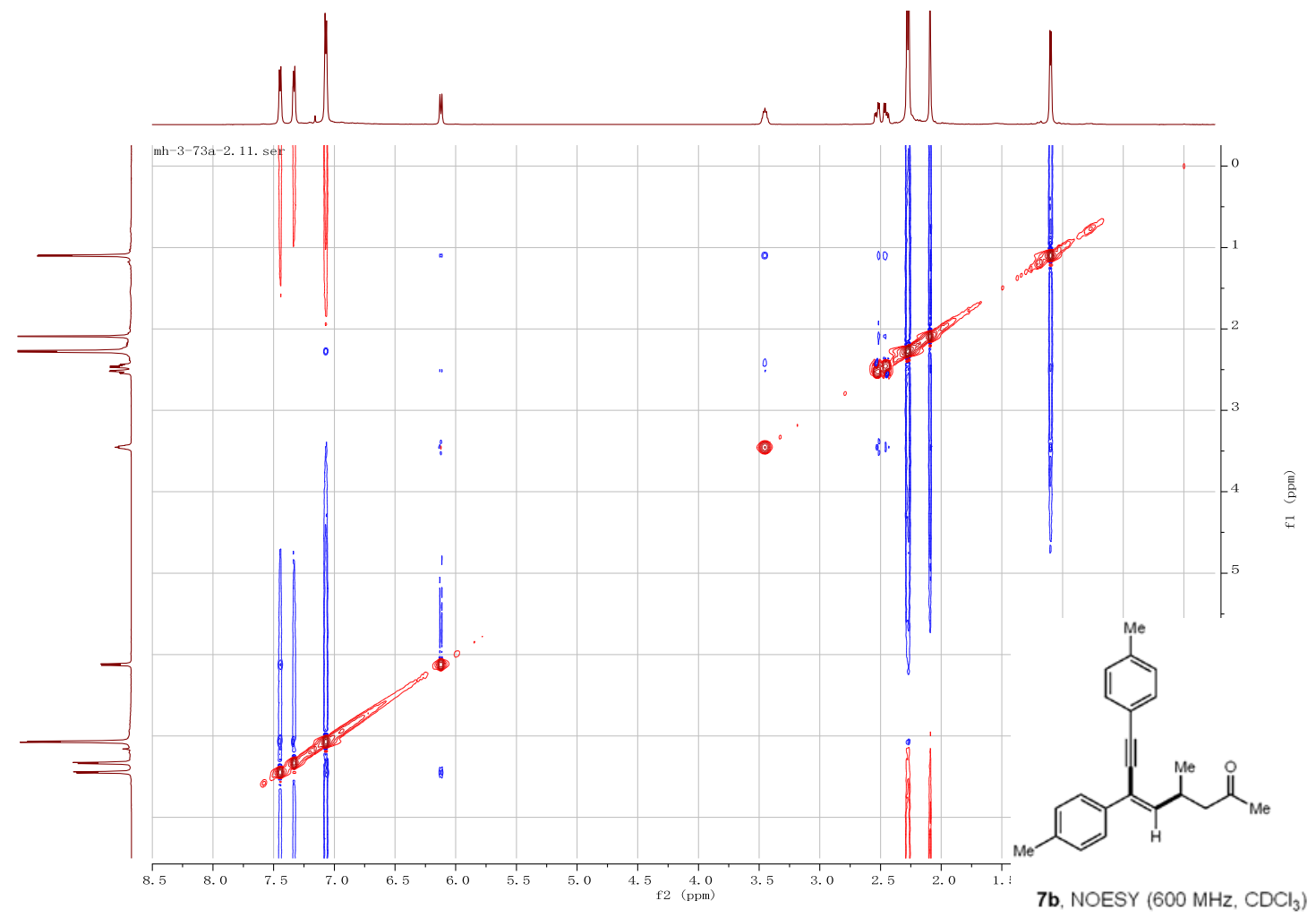

(Z)-4-Methyl-6,8-di-m-tolyloct-5-en-7-yn-2-one (7c)

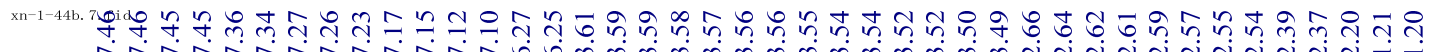<smiles>Cc1cccc(C#C/C(=C\C(C)CC(N)=O)c2cccc([N+](=O)[O-])c2)c1</smiles>

${ }^{1} \mathrm{H} \mathrm{NMR}\left(400 \mathrm{MHz}, \mathrm{CDCl}_{3}\right)$

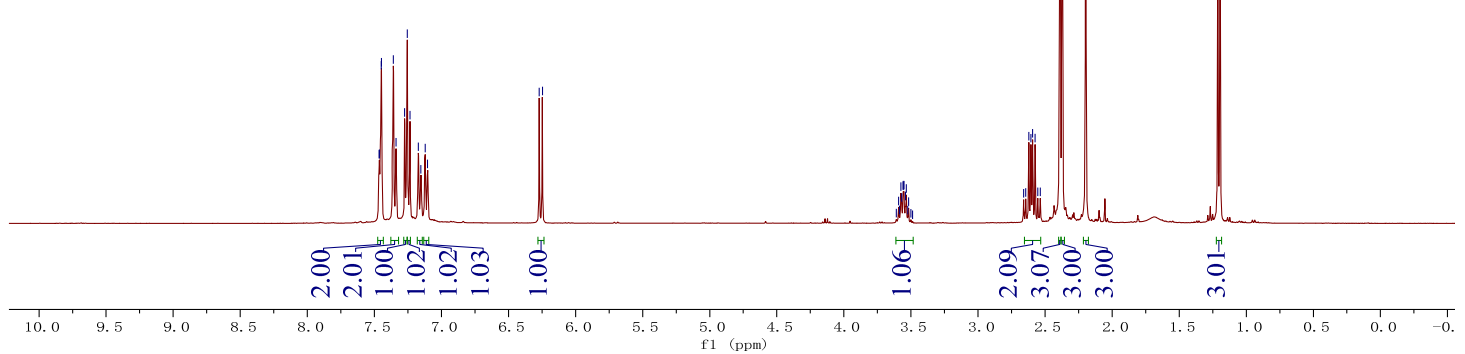




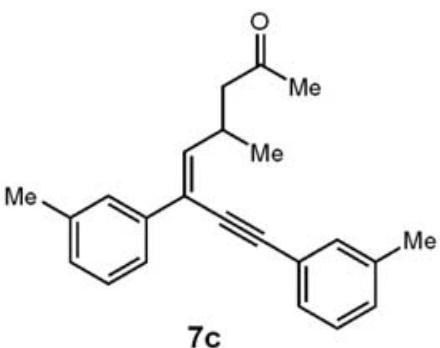

${ }^{13} \mathrm{C}$ NMR $\left(101 \mathrm{MHz}, \mathrm{CDCl}_{3}\right)$
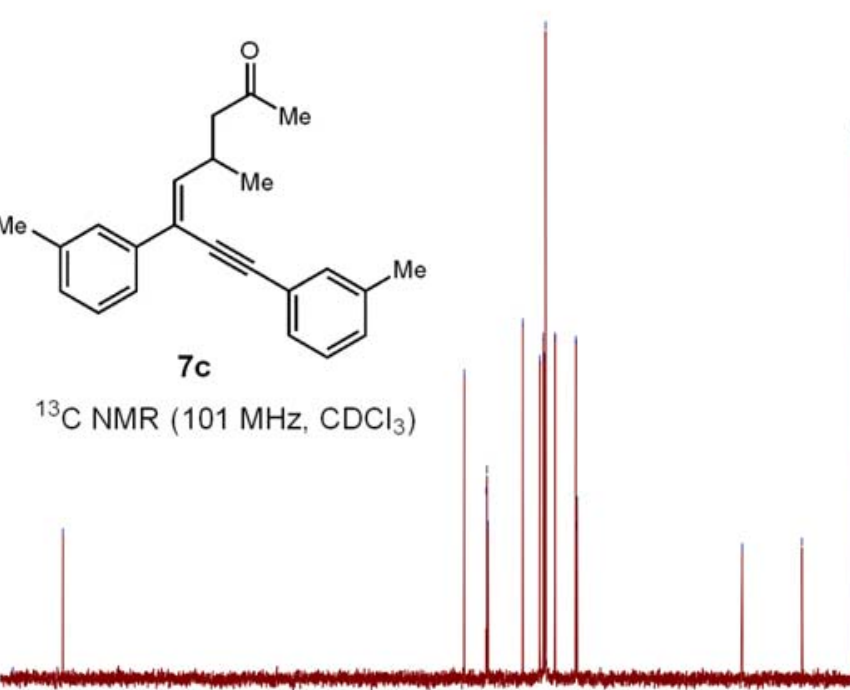

$\begin{array}{lllllll}210 & 200 & 190 & 180 & 170 & 160 & 150\end{array}$

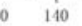

$\mathrm{fl}(\mathrm{ppon})$

(Z)-6,8-Bis(4-chlorophenyl)-4-methyloct-5-en-7-yn-2-one (7d)

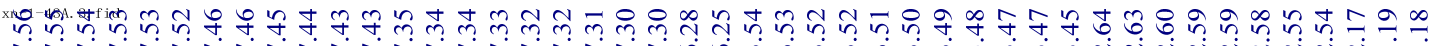

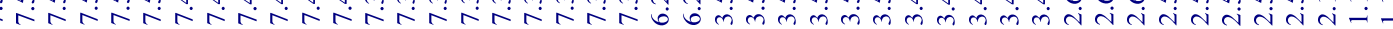<smiles>CC(=O)CC(C)/C=C(\C#Cc1ccc(Cl)cc1)c1ccc(Cl)cc1</smiles>

${ }^{1} \mathrm{H}$ NMR $\left(400 \mathrm{MHz}, \mathrm{CDCl}_{3}\right)$

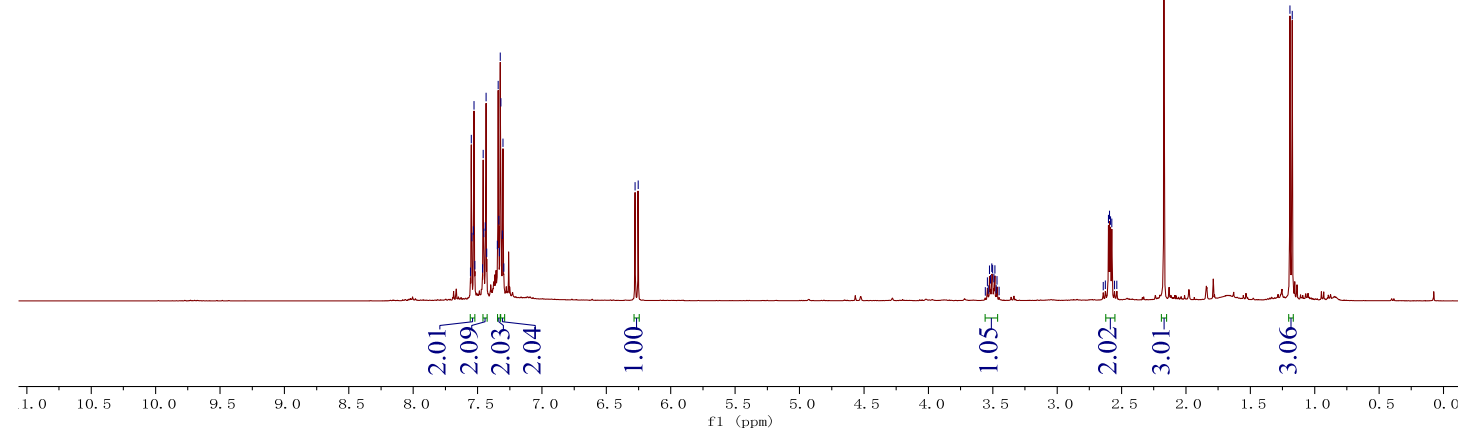


<smiles>CC(=O)CC(C)C=C(C#Cc1ccc(Cl)cc1)c1ccc(Cl)cc1</smiles>

${ }^{13} \mathrm{C}$ NMR $\left(101 \mathrm{MHz}, \mathrm{CDCl}_{3}\right)$
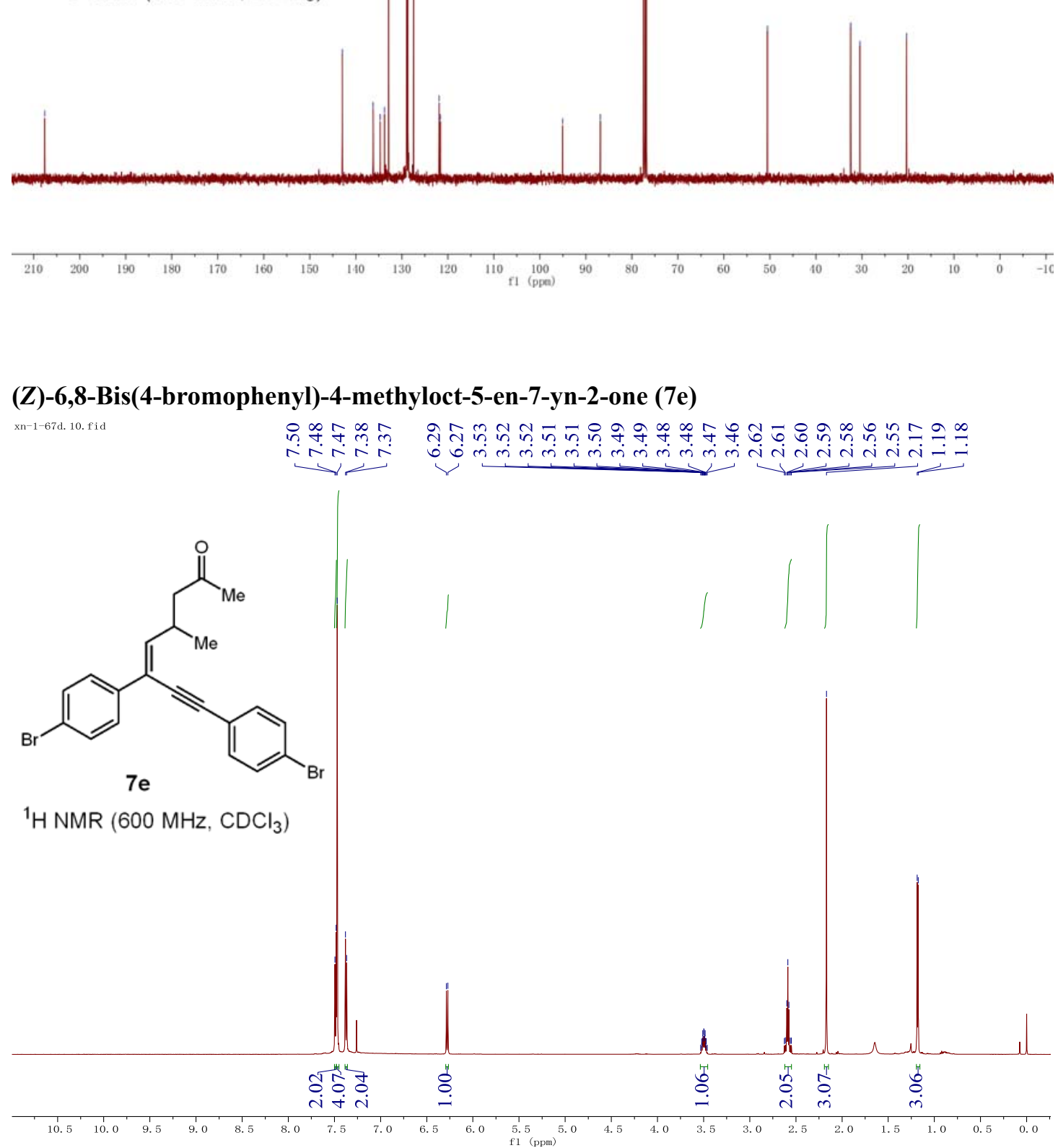


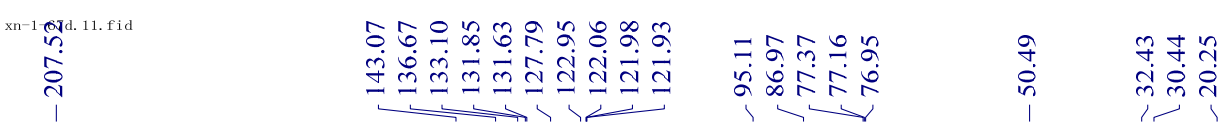<smiles>CC(=O)CC(C)/C=C(\C#Cc1ccc(Br)cc1)c1ccc(Br)cc1</smiles>

${ }^{13} \mathrm{C} \mathrm{NMR}\left(151 \mathrm{MHz}, \mathrm{CDCl}_{3}\right)$

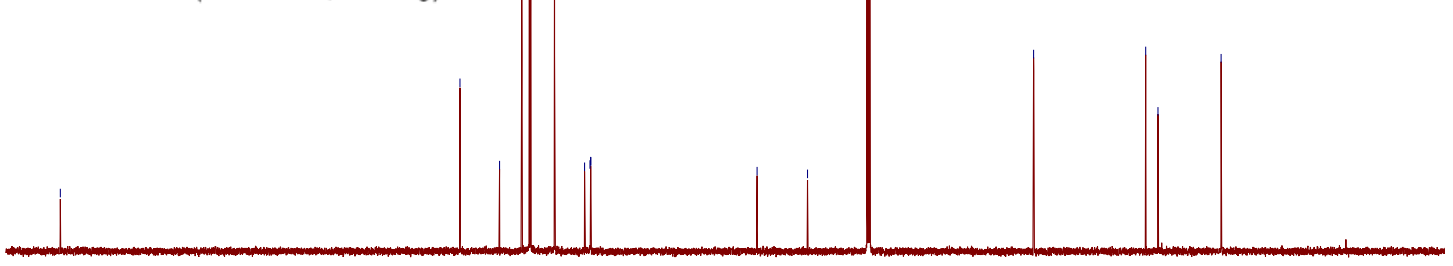

(Z)-6,8-Bis(4-fluorophenyl)-4-methyloct-5-en-7-yn-2-one (7f)

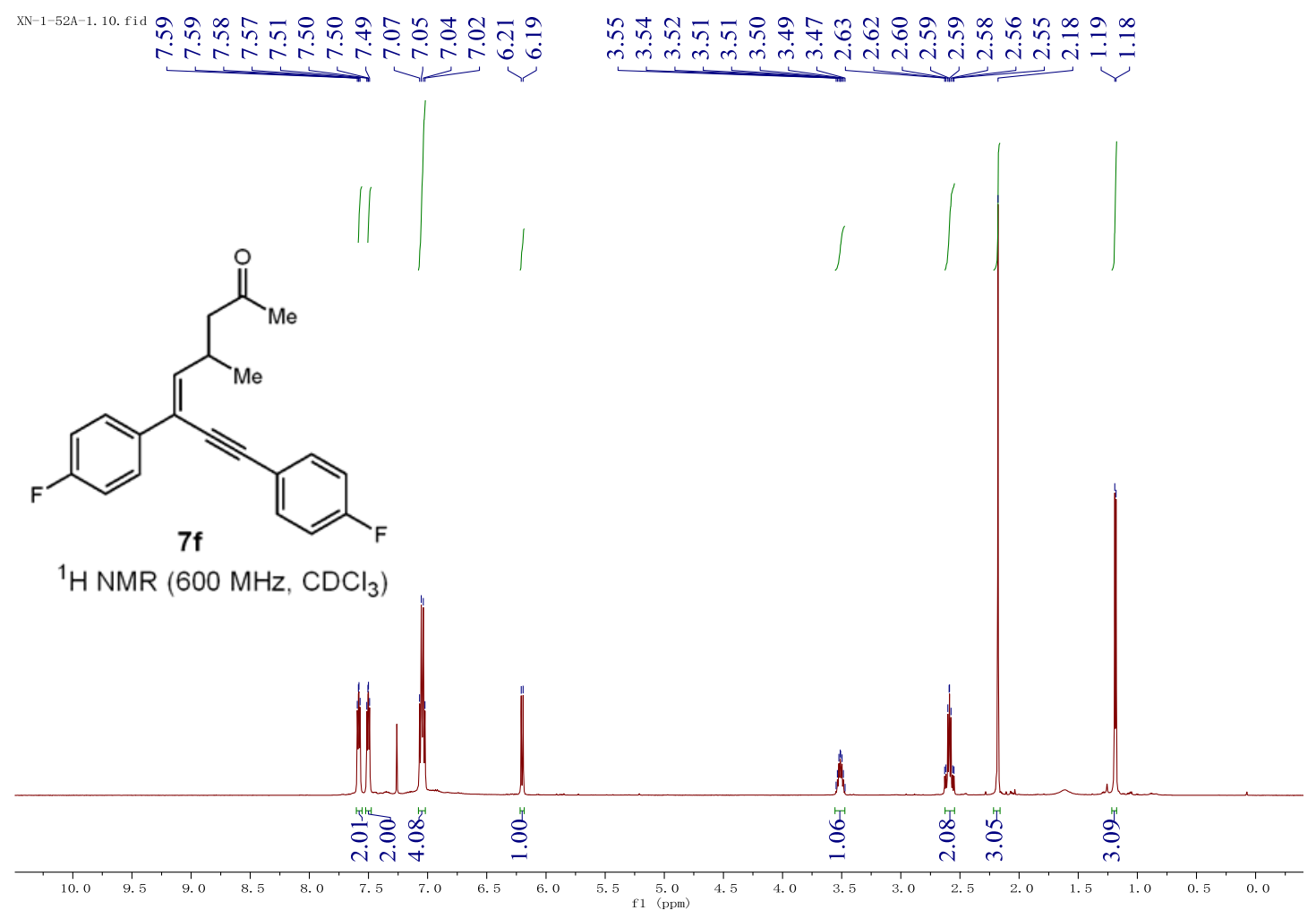




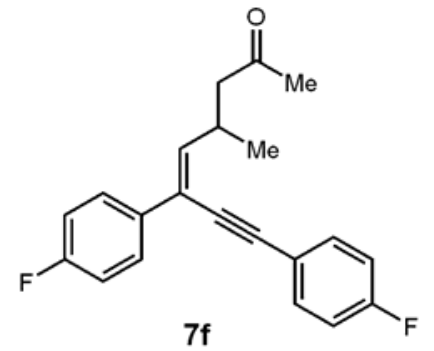

${ }^{19} \mathrm{~F} \mathrm{NMR} \mathrm{(376} \mathrm{MHz,} \mathrm{CDCl}_{3}$ )

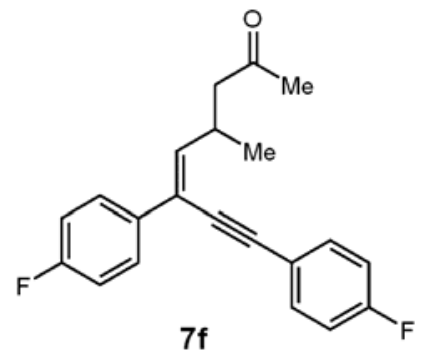

${ }^{13} \mathrm{C}$ NMR $\left(151 \mathrm{MHz}, \mathrm{CDCl}_{3}\right)$

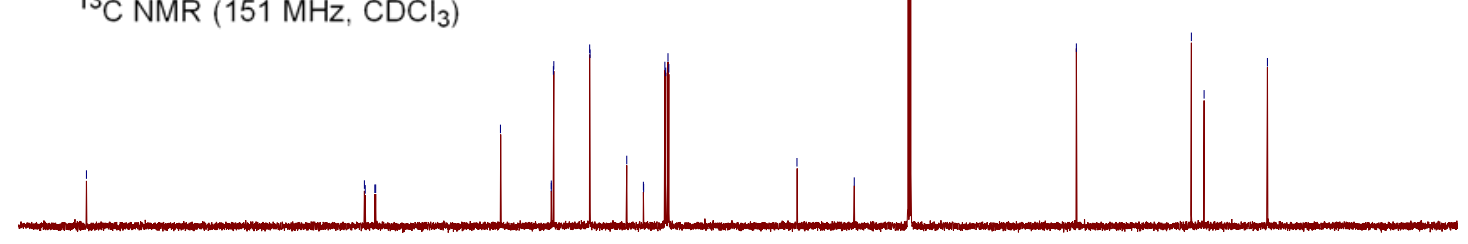


<smiles>CC(C=C(C#Cc1cccc(Cl)c1)c1cccc(Cl)c1)CC(N)=O</smiles>

${ }^{1} \mathrm{H} \mathrm{NMR}\left(400 \mathrm{MHz}, \mathrm{CDCl}_{3}\right)$

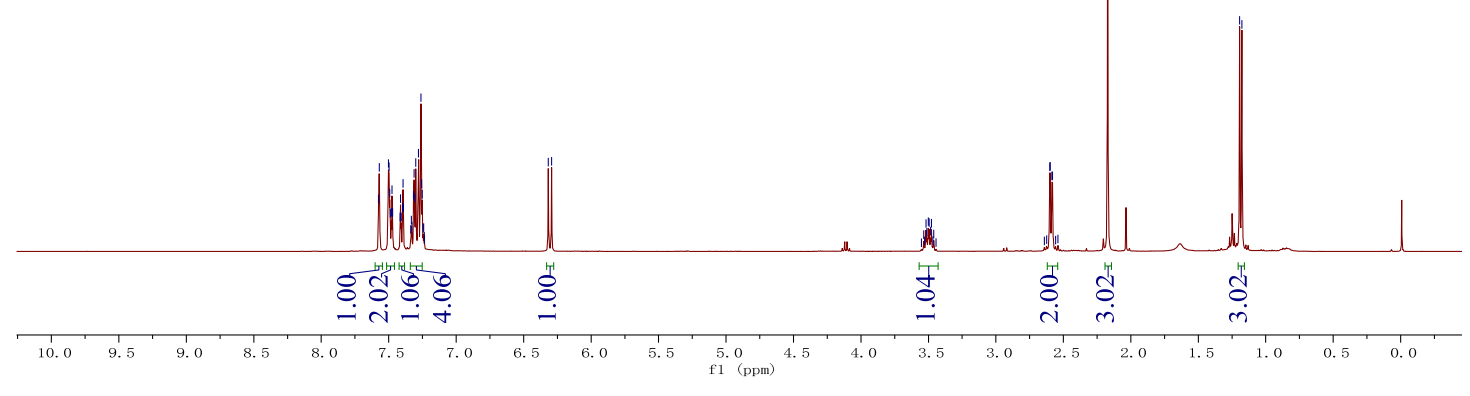<smiles>CC(=O)CC(C)/C=C(\C#Cc1cccc(Cl)c1)c1cccc(Cl)c1</smiles>

${ }^{13} \mathrm{C} \mathrm{NMR}\left(101 \mathrm{MHz}, \mathrm{CDCl}_{3}\right)$

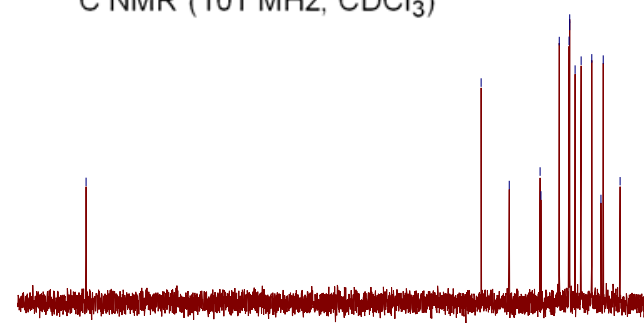




\section{(Z)-6,8-Bis(3-fluorophenyl)-4-methyloct-5-en-7-yn-2-one (7h)}

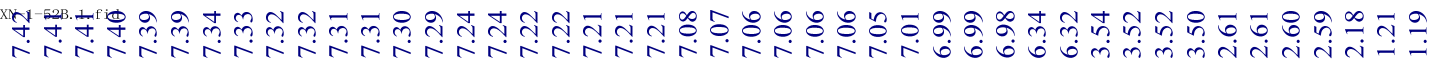<smiles>CC(=O)CC(C)/C=C(\C#Cc1cccc(F)c1)c1cccc(F)c1</smiles>

${ }^{1} \mathrm{H} \mathrm{NMR}\left(400 \mathrm{MHz}, \mathrm{CDCl}_{3}\right)$
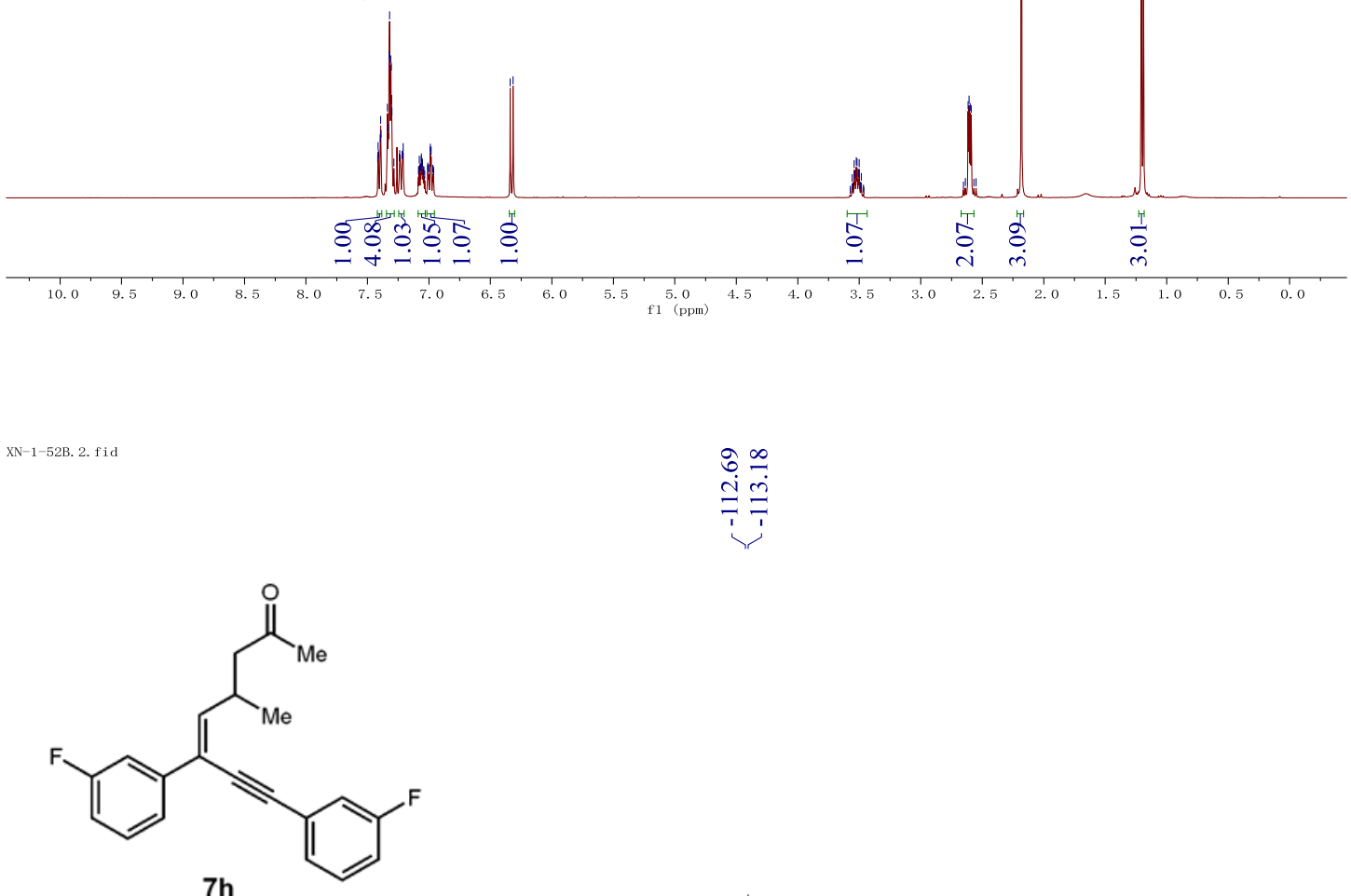

${ }^{19} \mathrm{~F} \mathrm{NMR}\left(376 \mathrm{MHz}, \mathrm{CDCl}_{3}\right)$

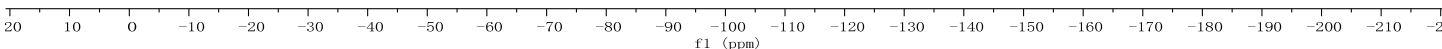




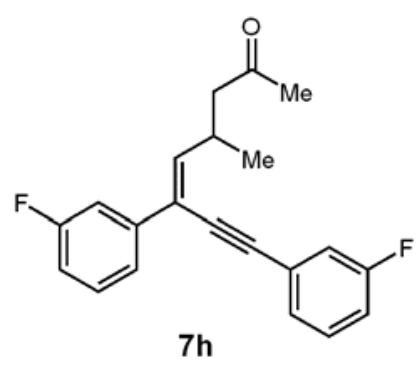

${ }^{13} \mathrm{C}$ NMR $\left(101 \mathrm{MHz}, \mathrm{CDCl}_{3}\right)$

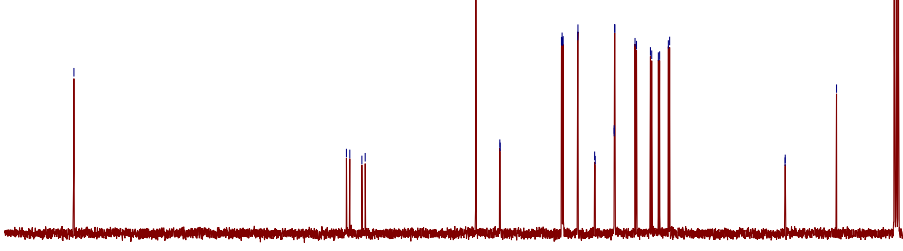

$\begin{array}{llllllllllll}1210 & 200 & 190 & 180 & 170 & 160 & 150 & 140 & 130 & 120 & 110 & 100 \\ \mathrm{f} 1 & (\mathrm{pmm})\end{array}$<smiles>CC(=O)CC(C)/C=C(/C#Cc1ccccc1F)c1ccccc1F</smiles>

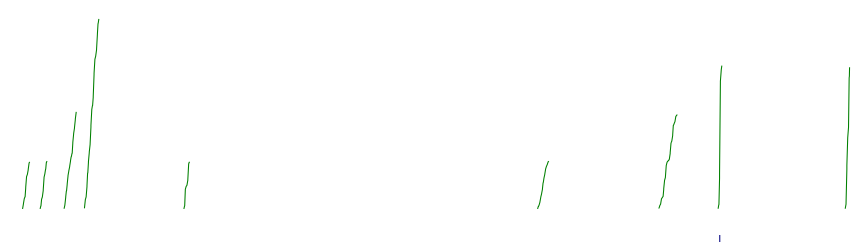

${ }^{1} \mathrm{H}$ NMR $\left(400 \mathrm{MHz}, \mathrm{CDCl}_{3}\right)$

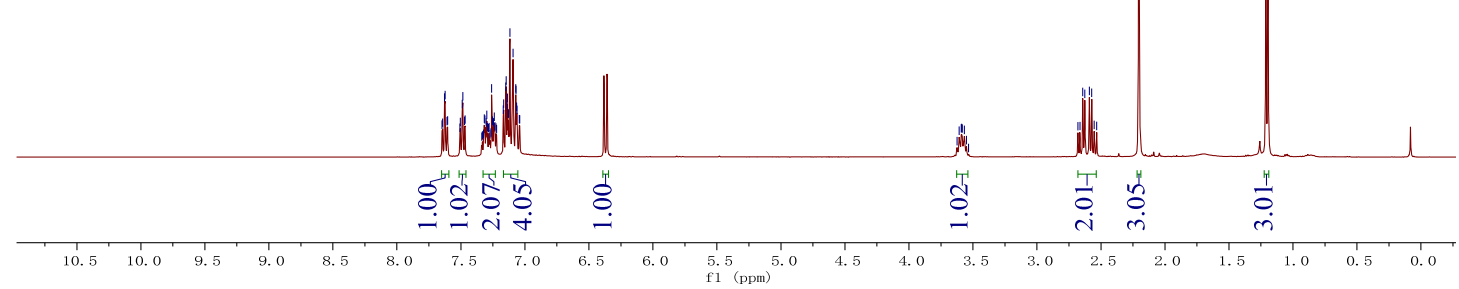




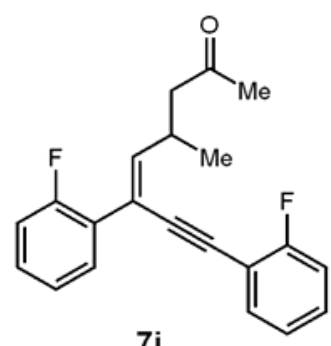

${ }^{19} \mathrm{~F} \mathrm{NMR}\left(376 \mathrm{MHz}, \mathrm{CDCl}_{3}\right)$

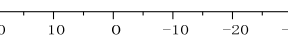<smiles>CC(=O)CC(C)/C=C(\C#Cc1ccccc1F)c1ccccc1F</smiles>

${ }^{13} \mathrm{C} \mathrm{NMR}\left(101 \mathrm{MHz}, \mathrm{CDCl}_{3}\right)$

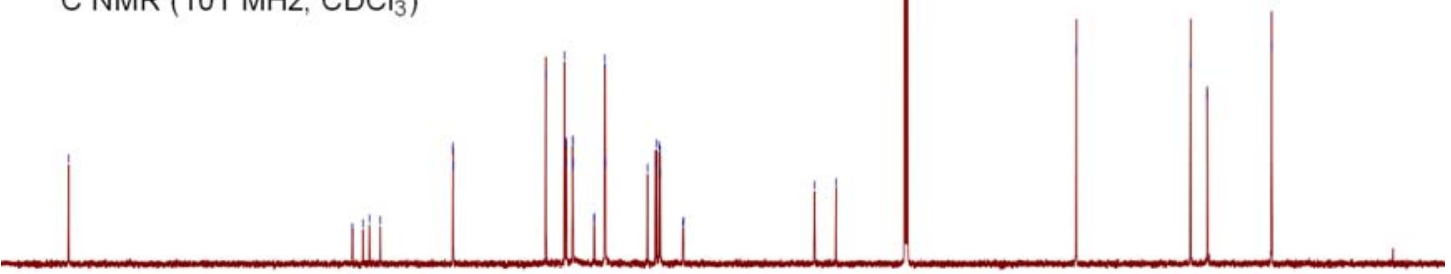

$\begin{array}{llllllllllll}210 & 200 & 190 & 180 & 170 & 160 & 150 & 140 & 130 & 120 & \underset{f 1}{110} 100\end{array}$ 


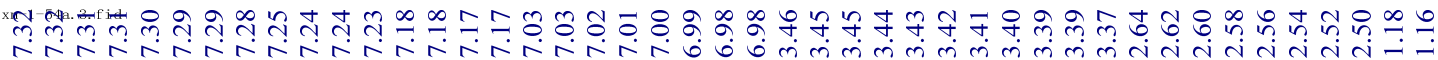<smiles>CC(=O)CC(C)/C=C(\C#Cc1cccs1)c1cccs1</smiles><smiles>C1=CC=C1</smiles>

${ }^{1} \mathrm{H} \mathrm{NMR}\left(400 \mathrm{MHz}, \mathrm{CDCl}_{3}\right)$
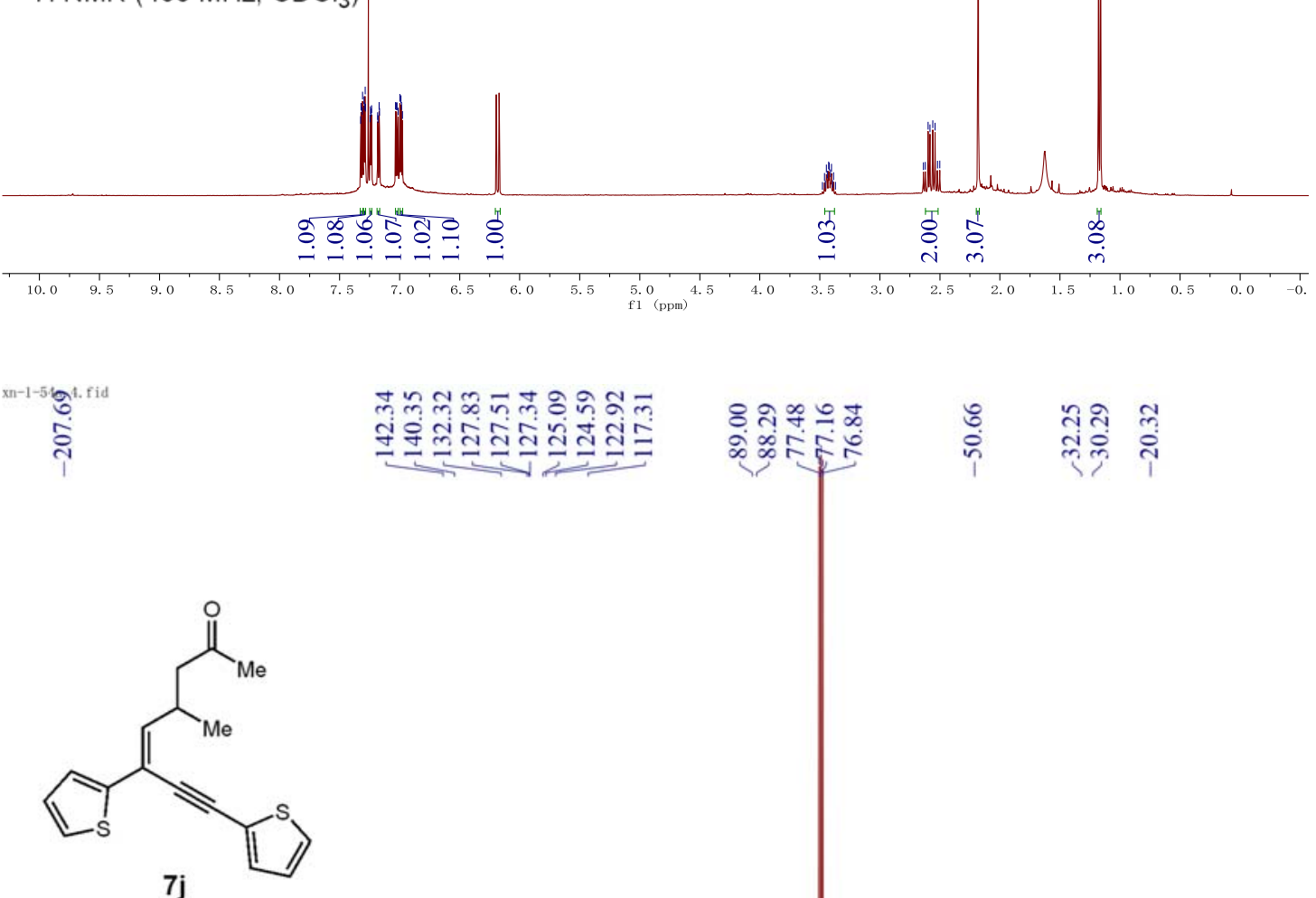

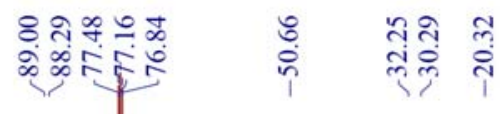

${ }^{13} \mathrm{C} \mathrm{NMR}\left(101 \mathrm{MHz}, \mathrm{CDCl}_{3}\right)$
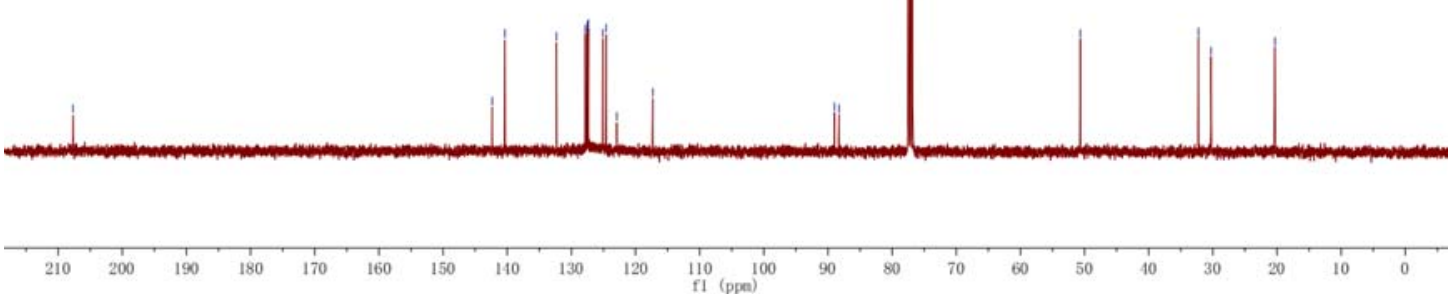


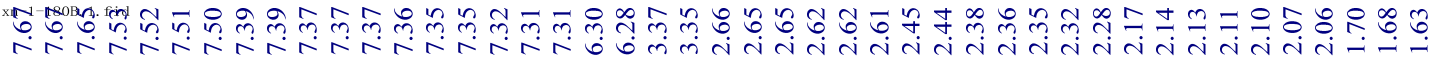<smiles>O=C1CCCC(C=C(C#Cc2ccccc2)c2ccccc2)C1</smiles>

$7 \mathrm{k}$

${ }^{1} \mathrm{H} \mathrm{NMR}\left(400 \mathrm{MHz}, \mathrm{CDCl}_{3}\right)$

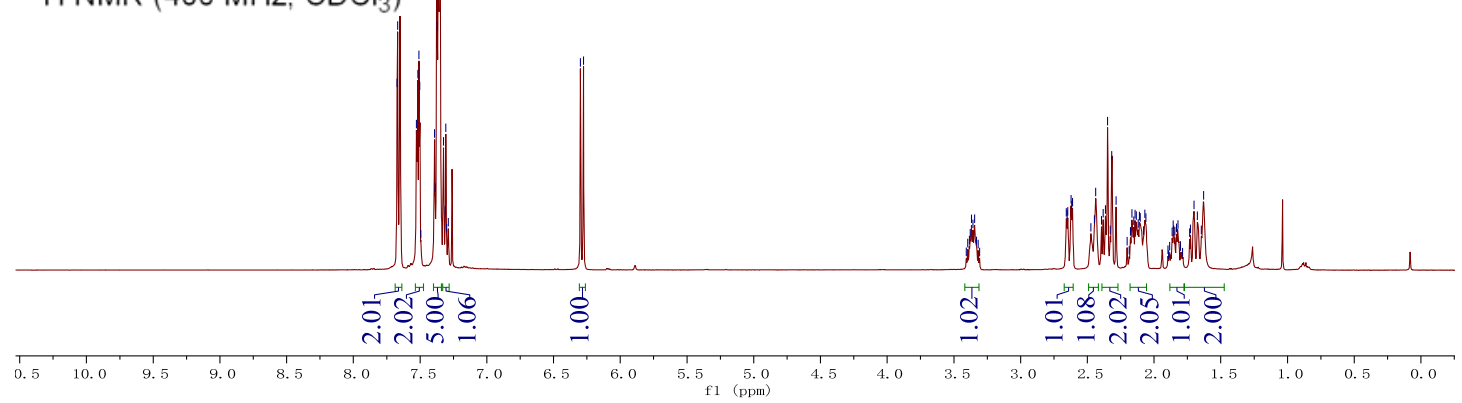<smiles>O=C1CCCC(C=C(C#Cc2ccccc2)c2ccccc2)C1</smiles>

$7 \mathrm{k}$

${ }^{13} \mathrm{CNMR}\left(101 \mathrm{MHz}, \mathrm{CDCl}_{3}\right)$

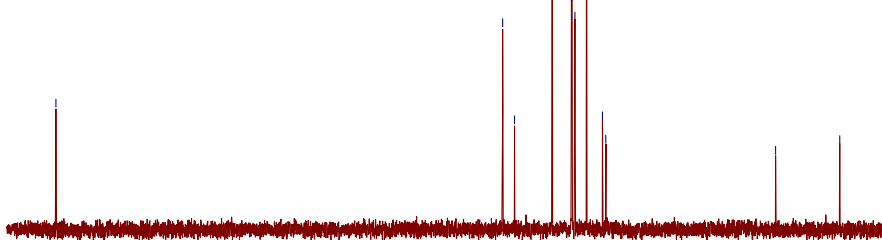




\section{(Z)-3-(2,4-Diphenylbut-1-en-3-yn-1-yl)cycloheptan-1-one (7l)}

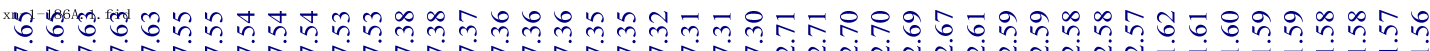

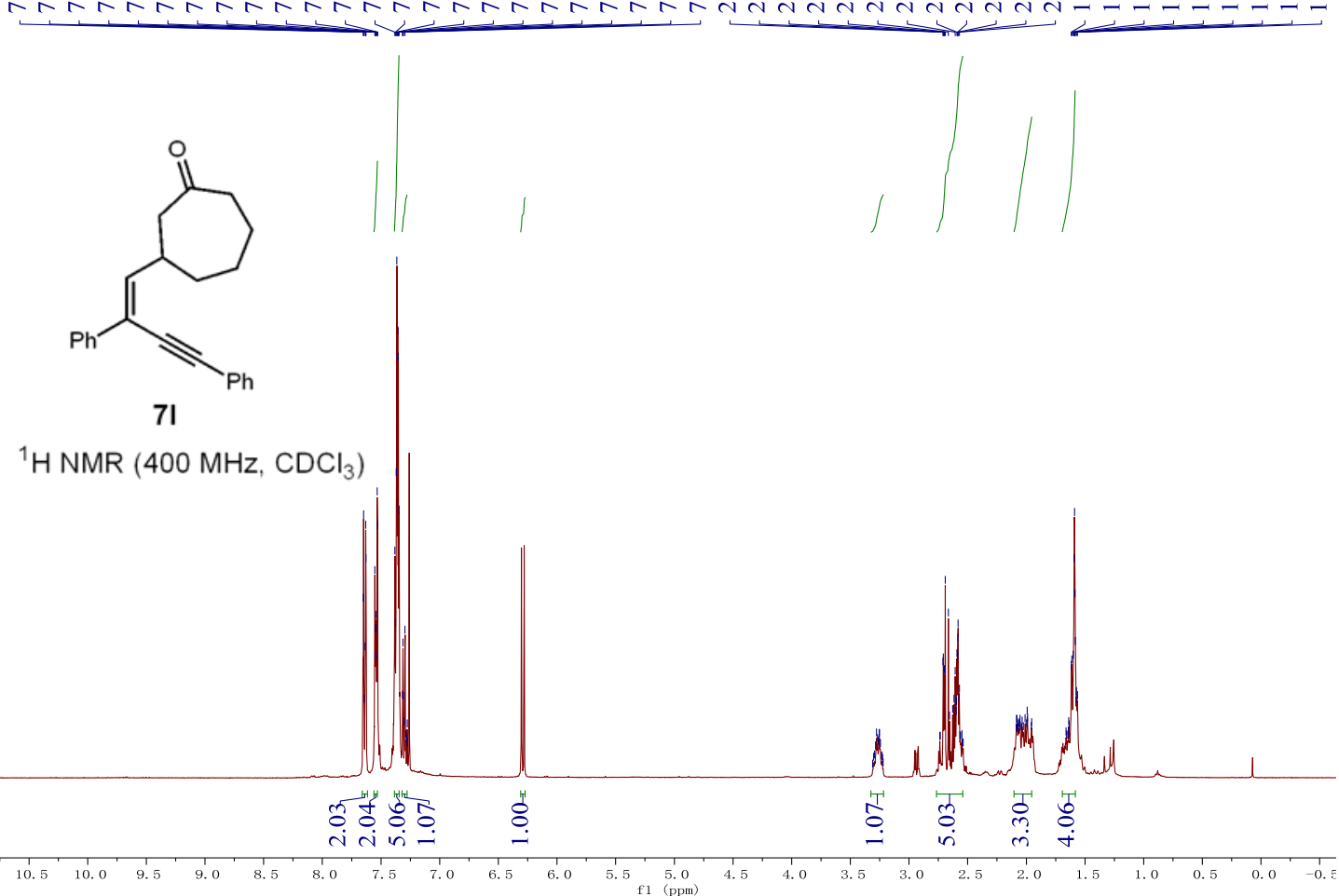

\begin{tabular}{|c|c|c|c|}
\hline 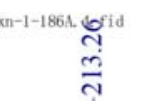 & 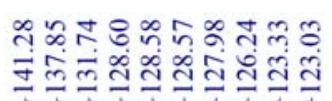 & 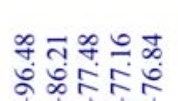 & 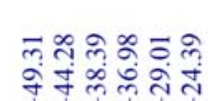 \\
\hline
\end{tabular}<smiles>O=C1CCCCC(C=C(C#Cc2ccccc2)c2ccccc2)C1</smiles>

71

${ }^{13} \mathrm{C} \mathrm{NMR}\left(101 \mathrm{MHz}, \mathrm{CDCl}_{3}\right)$

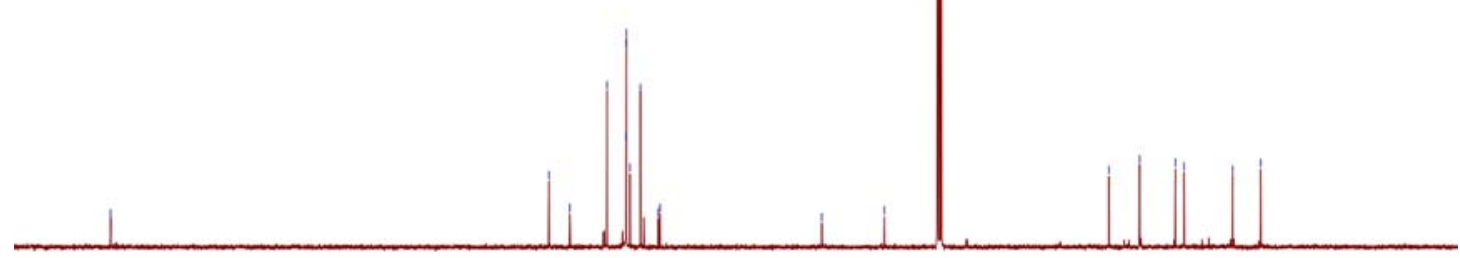

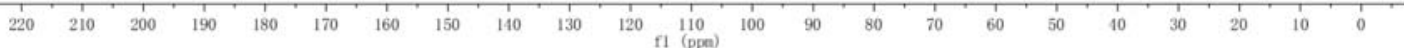




\section{(Z)-3-Methyl-5,7-diphenylhept-4-en-6-ynal (7m)}
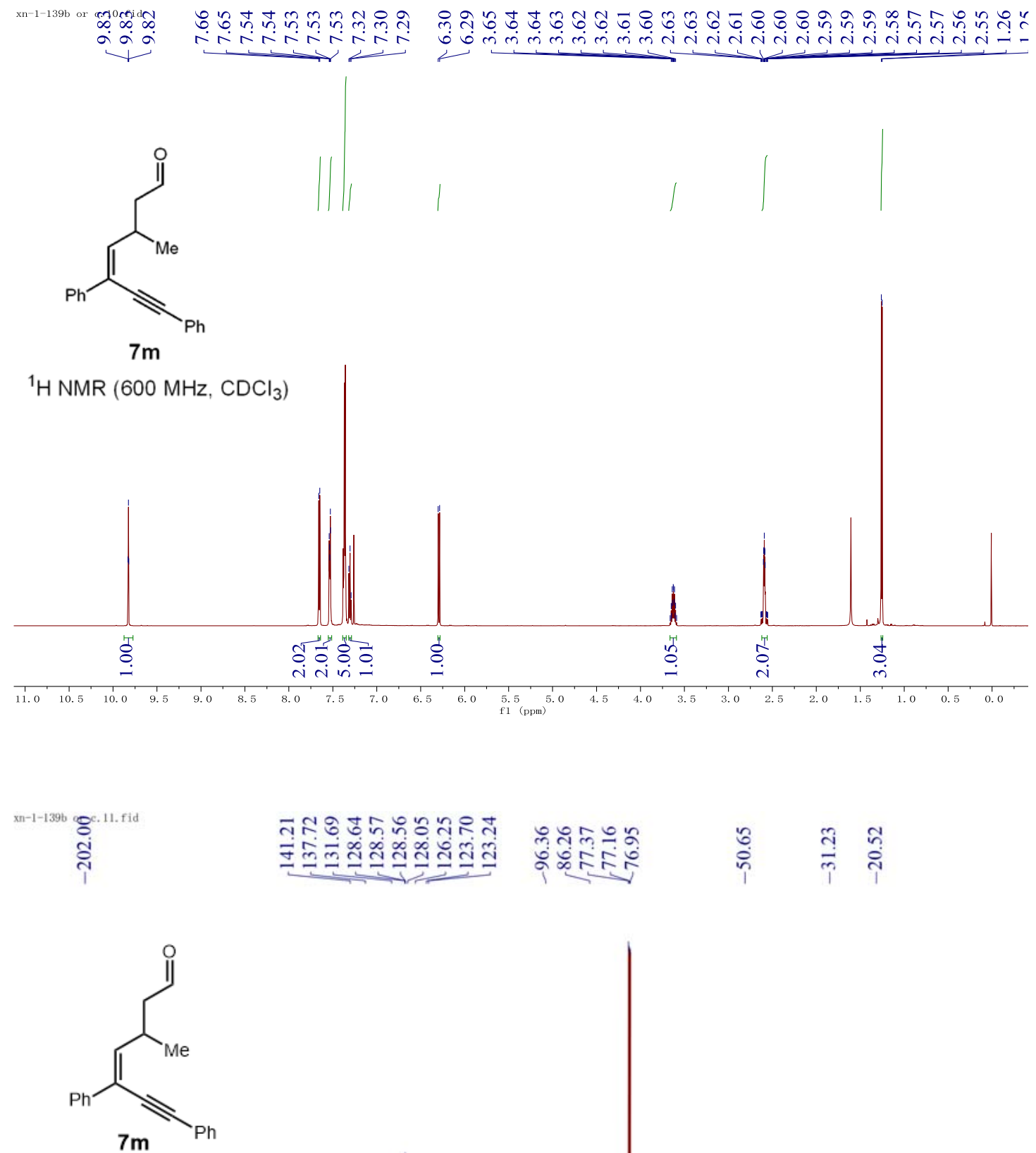

${ }^{13} \mathrm{C}$ NMR $\left(151 \mathrm{MHz}, \mathrm{CDCl}_{3}\right)$
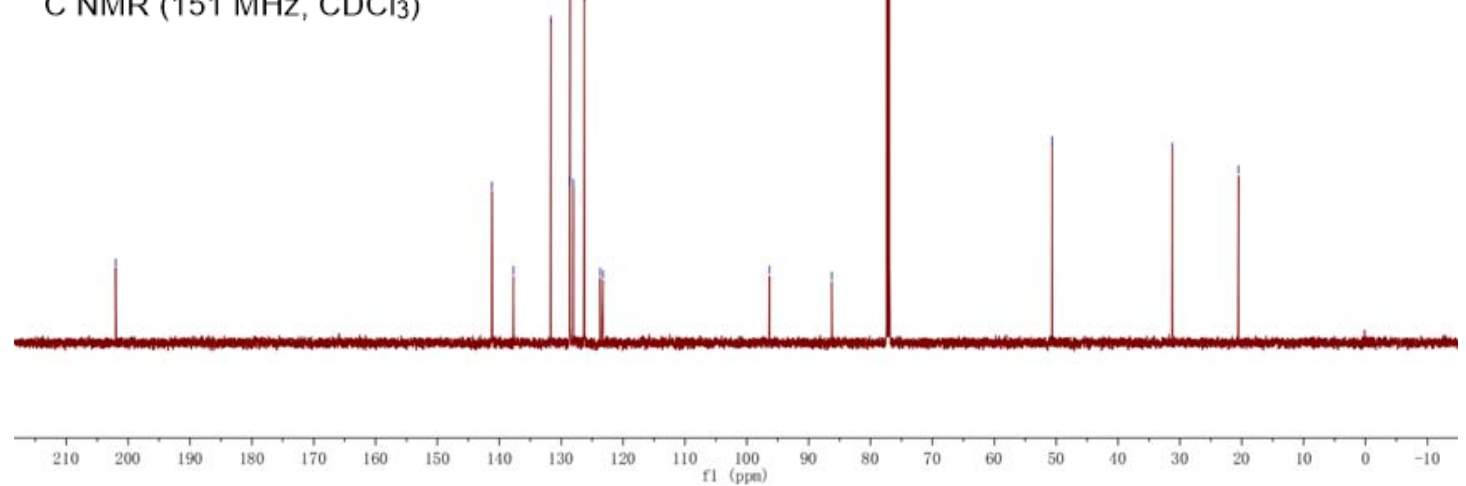


\section{(Z)-2-Methyl-5,7-diphenylhept-4-en-6-ynal (7n)}

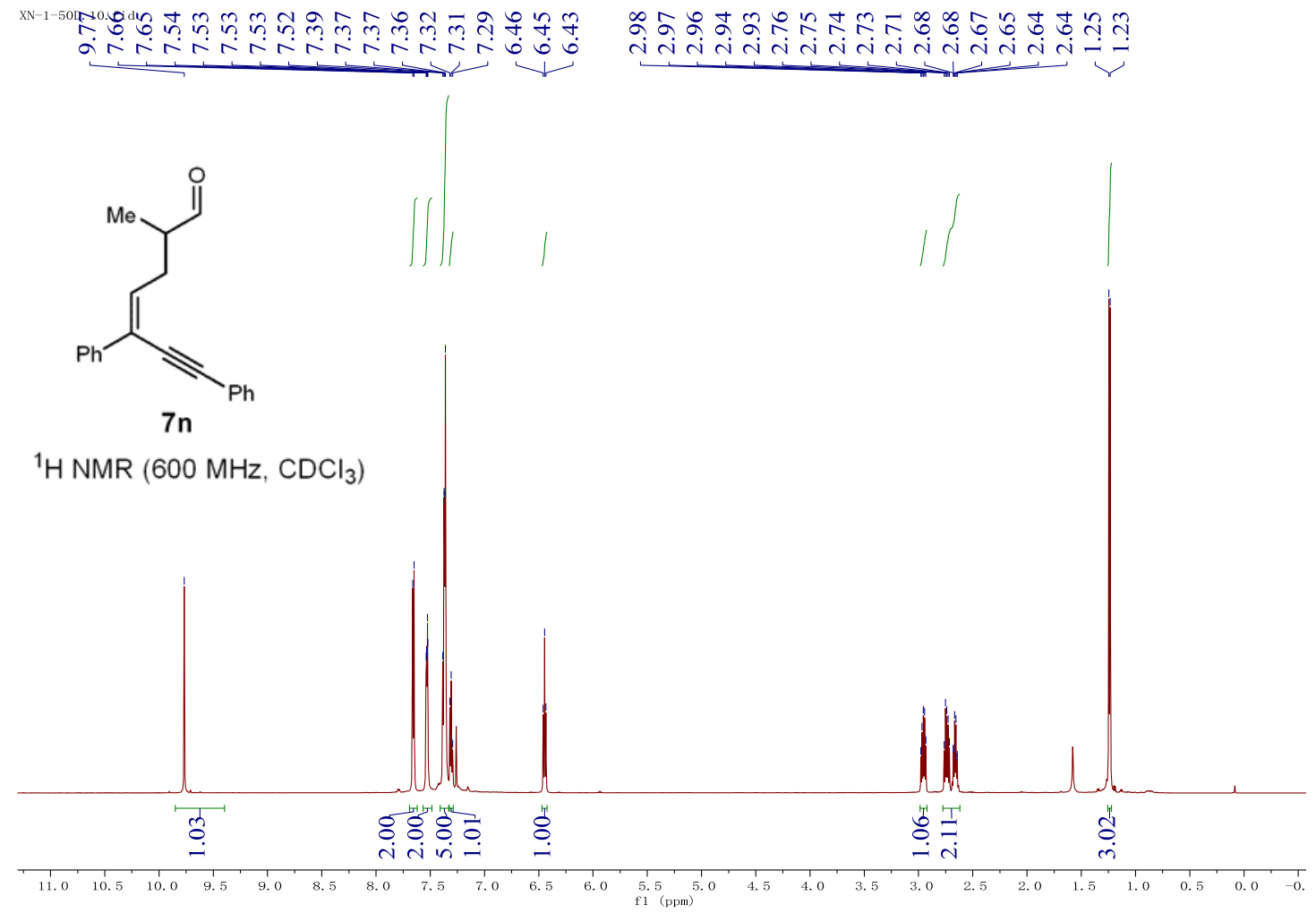

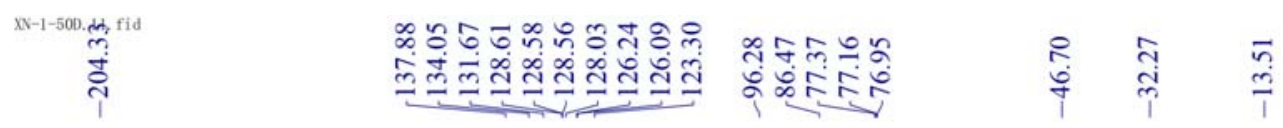

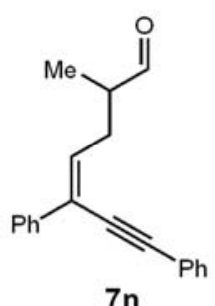

$7 \mathrm{n}$

${ }^{13} \mathrm{C} \mathrm{NMR}\left(151 \mathrm{MHz}, \mathrm{CDCl}_{3}\right)$
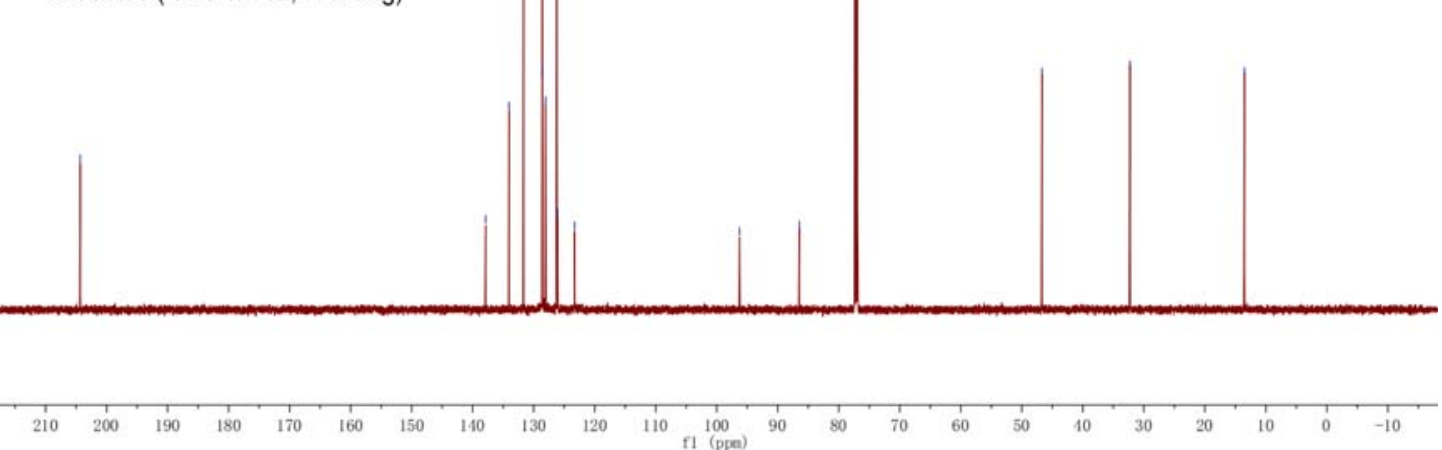
(Z)-6-(4-Methoxyphenyl)-4-methyl-8-phenyloct-5-en-7-yn-2-one-3,5- $d_{2}(8)$

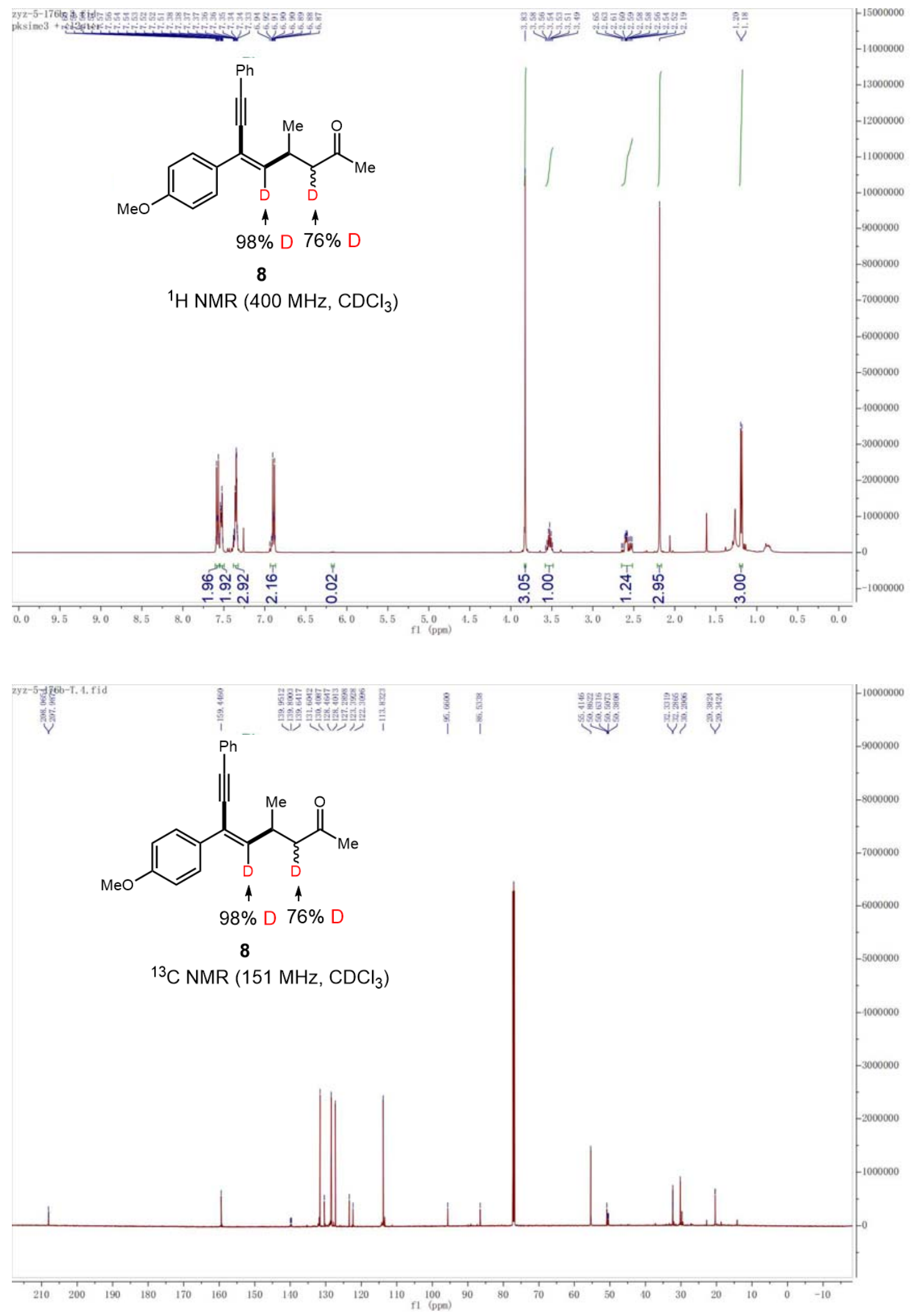


(Z)-6-(4-Methoxyphenyl)-4-methyl-8-phenyloct-5-en-7-yn-2-one-3-d (9)

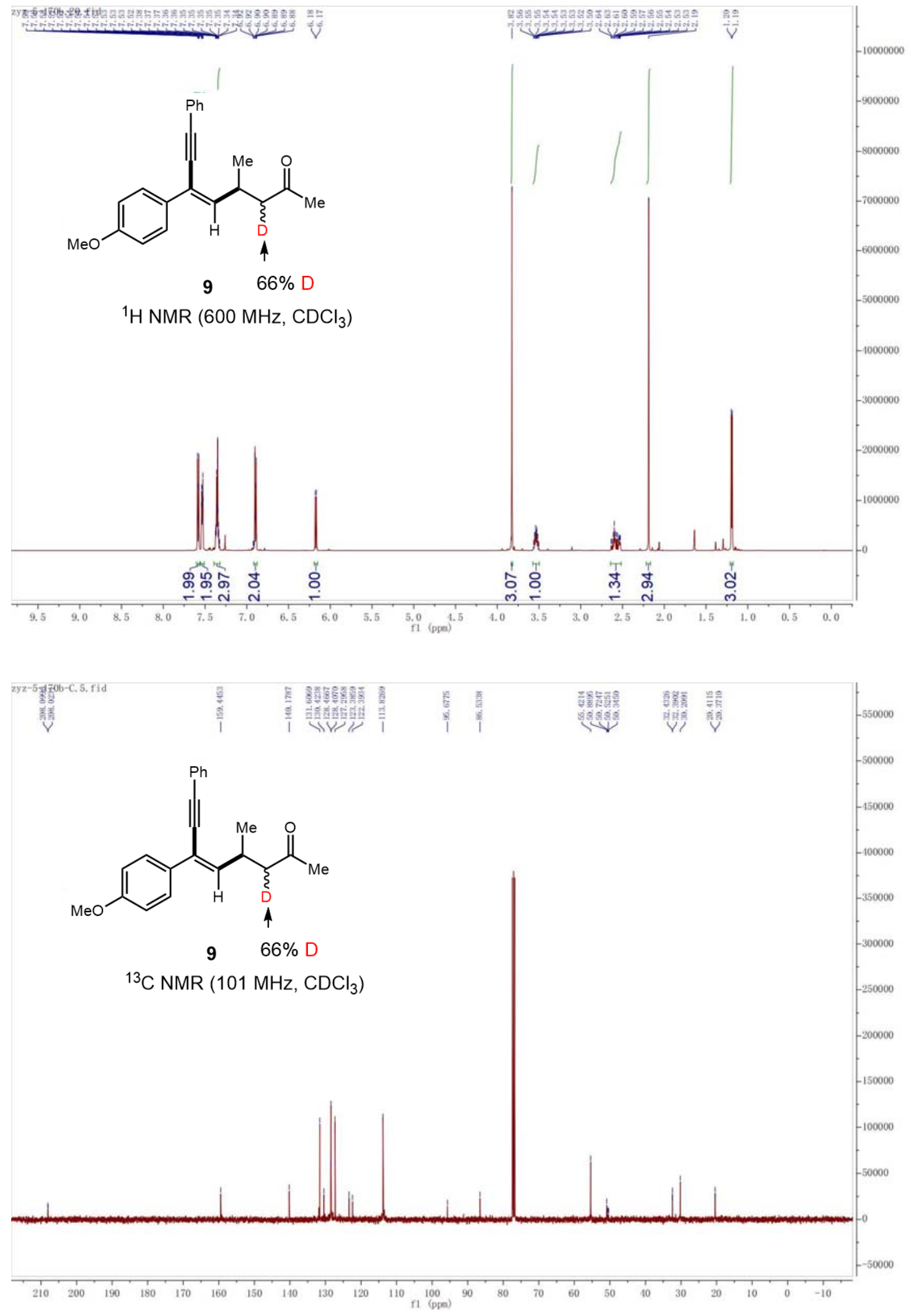


(Z)-6-(4-Methoxyphenyl)-4-methyl-8-phenyloct-5-en-7-yn-2-one-3,5-d 2 (10)
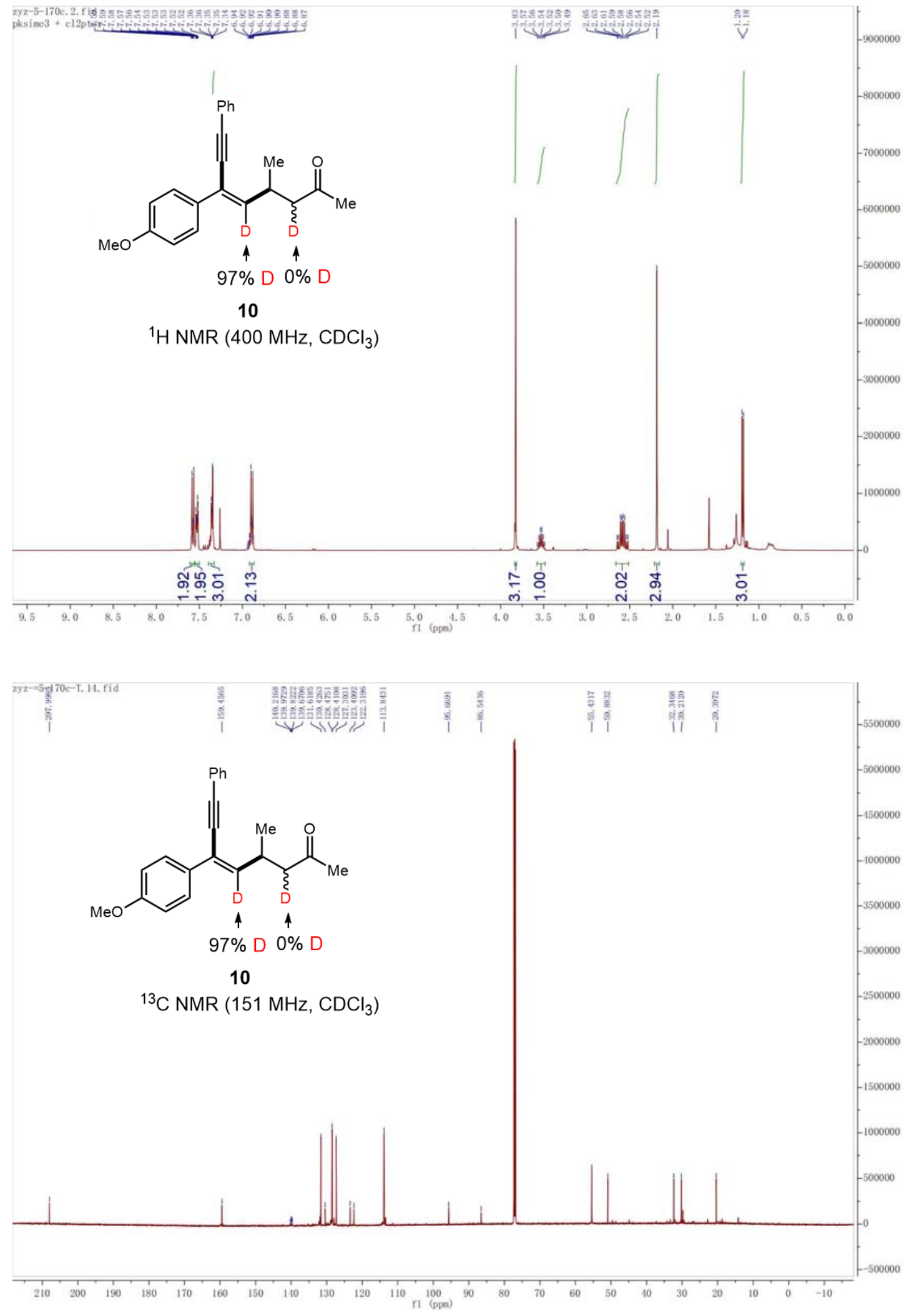
(Z)-6,8-Bis(4-methoxyphenyl)-4-methyloct-5-en-7-yn-2-one-1,3,5-d 3 (11)

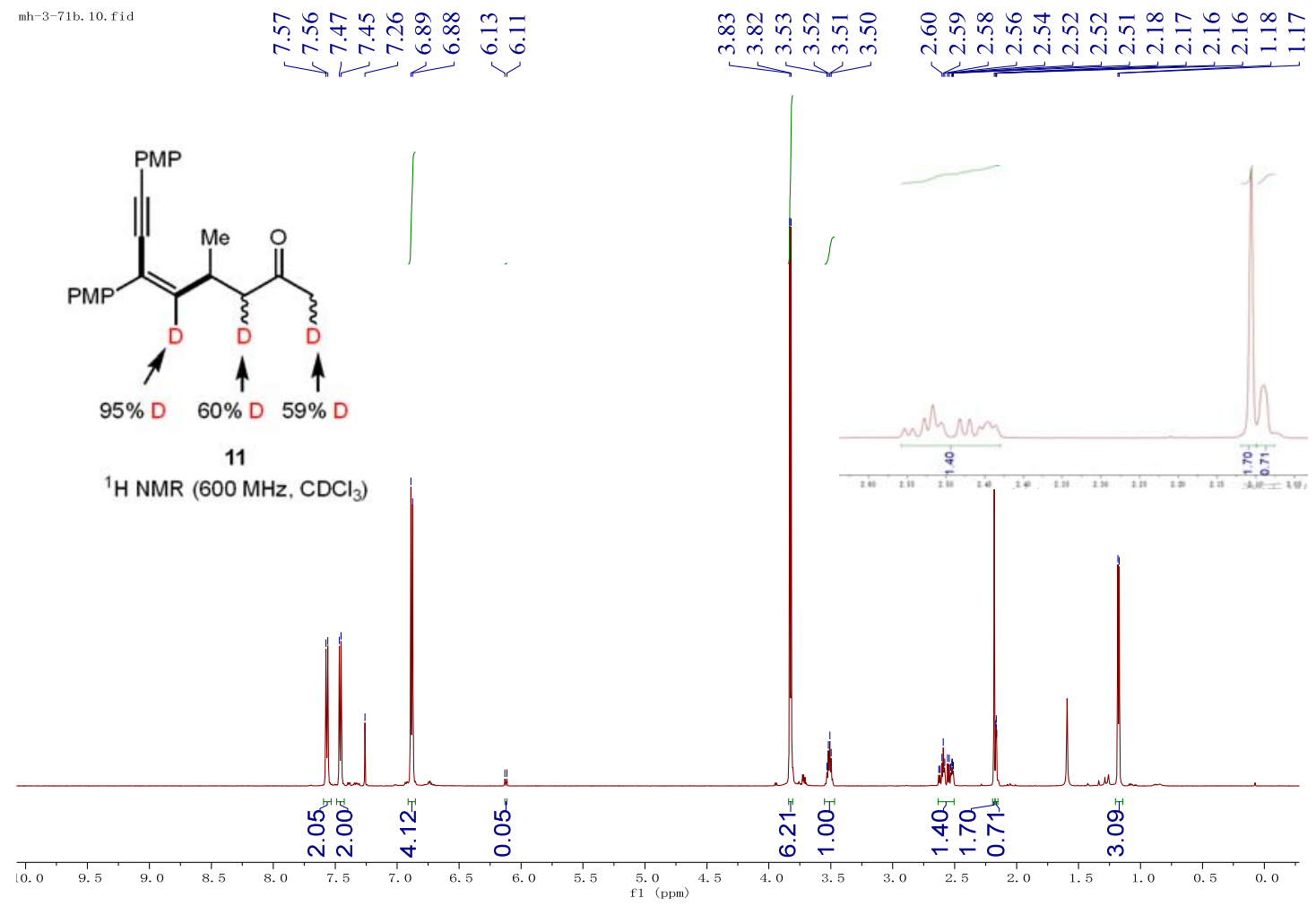

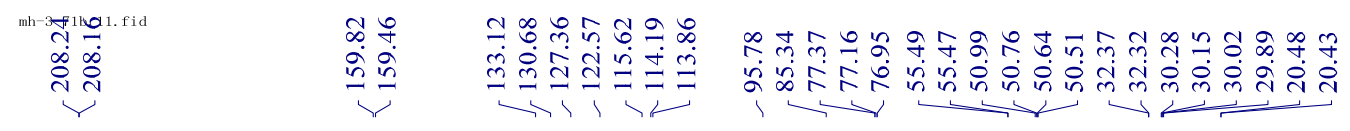

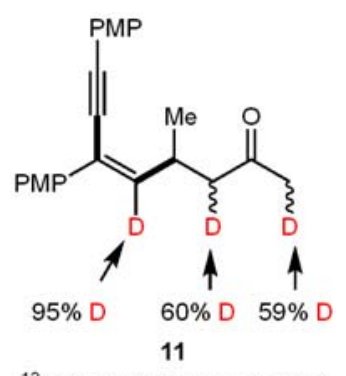

${ }^{13} \mathrm{C}$ NMR $\left(151 \mathrm{MHz}, \mathrm{CDCl}_{3}\right)$

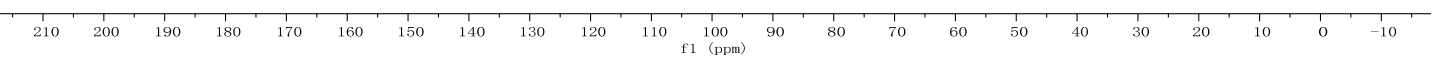


8. X-Ray Diffraction Data of 71 (CCDC 2086578)

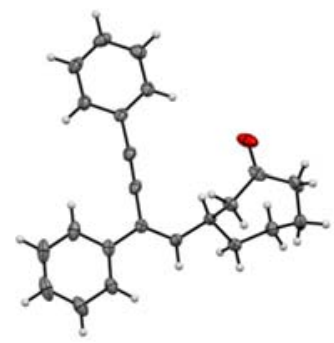

Table S13. Crystal data and structure refinement for 71 (cxy1700).

Identification code

Empirical formula

Formula weight

Temperature/K

Crystal system

Space group

$\mathrm{a} / \AA$

$\mathrm{b} / \AA$

$\mathrm{c} / \AA$

$\alpha /{ }^{\circ}$

$\beta /{ }^{\circ}$

$\gamma /{ }^{\circ}$

Volume $/ \AA^{3}$

Z

$\rho_{\text {calcg }} / \mathrm{cm}^{3}$

$\mu / \mathrm{mm}^{-1}$

$\mathrm{F}(000)$

Crystal size $/ \mathrm{mm}^{3}$

Radiation

$2 \Theta$ range for data collection/ ${ }^{\circ}$

Index ranges

Reflections collected

Independent reflections

Data/restraints/parameters

Goodness-of-fit on $\mathrm{F}^{2}$

Final $\mathrm{R}$ indexes $[\mathrm{I}>=2 \sigma(\mathrm{I})]$

Final $\mathrm{R}$ indexes [all data]

Largest diff. peak/hole / e $\AA^{-3}$ cxy 1700

$\mathrm{C}_{23} \mathrm{H}_{22} \mathrm{O}$

314.40

100.0

orthorhombic

Pben

$10.6227(5)$

$13.1948(6)$

$25.0235(11)$

90

90

90

$3507.4(3)$

8

1.191

0.071

1344.0

$0.38 \times 0.35 \times 0.28$

$\operatorname{MoK} \alpha(\lambda=0.71073)$

4.922 to 56.734

$-14 \leq \mathrm{h} \leq 14,-17 \leq \mathrm{k} \leq 17,-33 \leq 1 \leq 33$

33089

$4391\left[\mathrm{R}_{\text {int }}=0.0669, \mathrm{R}_{\text {sigma }}=0.0334\right]$

$4391 / 0 / 218$

1.028

$\mathrm{R}_{1}=0.0512, \mathrm{wR}_{2}=0.1170$

$\mathrm{R}_{1}=0.0697, \mathrm{wR}_{2}=0.1284$

$0.36 /-0.23$ 